\title{
Characterisation of Juvenile Hormone Esterase in Drosophila melanogaster
}

by

\section{Peter Malcolm Campbell}

Division of Biochemistry and Molecular Biology,

Faculty of Science, Australian National University

and

C.S.I.R.O. Division of Entomology,

Canberra, Australia.

A thesis submitted for the degree of Doctor of Philosophy

of The Australian National University, August 1993. 


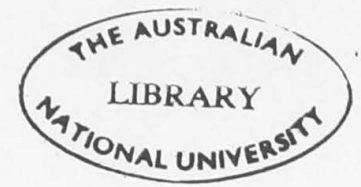

\section{Declaration}

The research carried out in the course of this investigation and the results presented in this thesis are, except where acknowledged, the original work of the author.

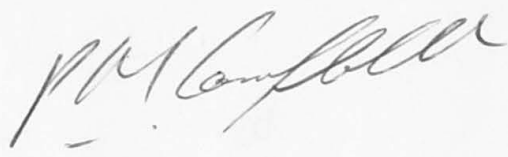

Peter Malcolm Campbell 


\section{Acknowledgements}

I would like to thank my supervisors Marion Healy and John Oakeshott of the Division of Entomology, CSIRO for their support and patience which extended far beyond the call of duty. Among other things this has contributed greatly to my ability to enjoy the first year of my son's life. Also, I would like to thank Tony Howells, my ANU supervisor.

The members of our laboratory, past and present, have been a wonderful group with whom to work and socialise. I appreciate the cooperative spirit which has prevailed and I thank them all. In particular, I would like to thank Richard, Lis, Wendy, and Charlie who each proof-read a chapter of this thesis.

I have received advice, suggestions and practical assistance from many members of the Division of Entomology. These have included Stephen Trowell, Terry Hanzlik and Peter East who share an interest in juvenile hormone. I have had useful conversations with the resident chemists, Tom Bellas and Mike Lacey. Chris Whittle assisted with the synthesis the affinity chromatography matrix and OTFP and Vance Brown synthesised diazomethane. Stephen Trowell kindly provided a sample of lipophorin purified in his laboratory. Thomas Boyce provided advice on the statistical analysis of my data and Peter Atkinson helped with the TLC detection method for JH metabolites. Chris Hunt, Sandy Smith, John Green and Eric Hines provided assistance with the preparation of figures.

Scientists from outside the Division of Entomology who have assisted include Virginia Walker of Queen's University, Canada who advised on several minor experiments during her visit to our laboratory. Richard Thomas and Rod Rickards of the Research School of Chemistry, ANU synthesised and generously provided many of the JH analogues used in this study. John Morrison of the John Curtin School of Medical Research, Australian National University performed the statistical analysis of the data from which Michaelis and inhibition constants were calculated.

I am grateful for the scholarship provided by the Australian Wool Corporation and for a further grant which enabled me to visit the laboratories of Dr. B.D. Hammock, Drs. G. and D. Jones and Dr. C. A. D. de Kort. Members of these laboratories provided helpful and interesting discussion. I have also grateful for correspondence from Drs. J. H. Willis and L.M. Riddiford on the topics of the anti-metamorphic role of JH and the physiology of $D$. melanogaster metamorphosis, respectively. my wife, Sue.

Finally, I would like to acknowledge the love, tolerance, patience and support of 
Table of Contents

Summary

\section{Chapter 1 Introduction and Literature Review}

1.1

Introduction

The Structures and Distribution of Juvenile Hormones

$1.3 \quad$ Functions of JH

Physiology and Hormonal Regulation of Insect Metamorphosis

Physiology of Metamorphosis

Hormonal Regulation of Metamorphosis

Mechanisms Underlying Hormonal Control of Metamorphosis

Timing of Metamorphosis Mechanisms for JH Action Mechanisms for Ecdysone Action

Regulation of JH Titre

Synthesis of JH

JH Carrier Proteins

JH Degradation

Definition of "JH-Specific" Hydrolysis

Physiology of JH Hydrolysis

Temporal Profile of JH hydrolysis

Spatial Profile of JH Hydrolysis

1.6 .3

Regulation of JH Hydrolysis

Biochemistry of Juvenile Hormone Esterases 


\section{Chapter 2 Identification and Expression Pattern of $\mathrm{JH}$ Degrading Enzymes}

Materials and Methods 26

2.2.1.1

Drosophila Strains, Culture and Sample Preparation

Strains and Culture

Sample Preparation

Procedures

2.2.2.2

Protein Determination

Native Polyacrylamide Gel Electrophoresis (PAGE)

2.2.2.3 Kinetic Experiments Using Native PAGE Isolation of JHE Activity After Native PAGE Thin Layer Chromatography to Identify JH Hydrolytic Products

Partition Assay of JH Hydrolytic Activities $\mathrm{pH}$, Isoelectric and Inhibition Properties of JHE 29

2.2.3.1

2.2.3.2 $\mathrm{pH}$ Profile of JHE Activity

\section{Comparison of the Anodal Esterases of Drosophila} melanogaster and Drosophila virilis

\section{Pattern of Expression of the Anodal Esterases}

Biochemical Properties of the Anodal Esterases

Characterisation of JH Hydrolysis

Localisation of JHE After Native PAGE 


\section{Chapter 3 Biochemical Characterisation of Purified JHE}

\section{1}

Introduction

3.2

3.2 .1

3.2 .2

3.2.2.1

3.2.2.2

3.2.2.3

3.2 .3

3.2.3.1

3.2.3.2

3.2.3.3

3.2 .4

3.2.4.1

3.2.4.2

3.2 .4 .3

3.2.4.4

3.3

3.3.1

3.3.2

3.3.2.1

3.3.2.2

3.3.2.3

3.3.2.4

3.3.3

3.3.3.1

3.3.3.2

3.3.3.3

3.3.3.4

3.4

Discussion

Materials and Methods

40

Chemicals

40

JHE Assay, Electrophoretic and Protein Detection

Procedures

JHE Activity Assay

40

Electrophoretic Procedures

40

Purification of JHE

Initial Purification Steps

41

Affinity Purification of JHE

41

Conventional Purification

42

Kinetic Characterisation of JHE

42

Kinetic Analysis of JHIII Hydrolysis and Inhibition

by JH Isoforms

Kinetic Properties with $\alpha$-Naphthylacetate

43

Identification of JH Structural Features

which Promote Binding to JHE

Interaction of JHE with Lipophorin

$\underline{\text { Results }}$

45

Purification of JHE $\quad 45$

Properties of Purified JHE 46

Identification of JHE by Two-Dimensional PAGE 46

Molecular Weight Estimation $\quad 47$

Stability of Purified JHE $\quad 47$

N-Terminal Sequencing $\quad 47$

Kinetic Characterisation of JHE 48

Kinetic Analysis of JHIII Hydrolysis and Its Inhibition by JH Isoforms 48

Kinetic Properties with $\alpha$-Naphthylacetate $\quad 48$

Binding Affinities for Other JH Analogues and Isoforms 48

Interaction of JHE with Lipophorin $\quad 52$

Purification and Physical Properties of JHE

Kinetic Characterisation of JHE 


\section{Chapter 4 General Discussion}

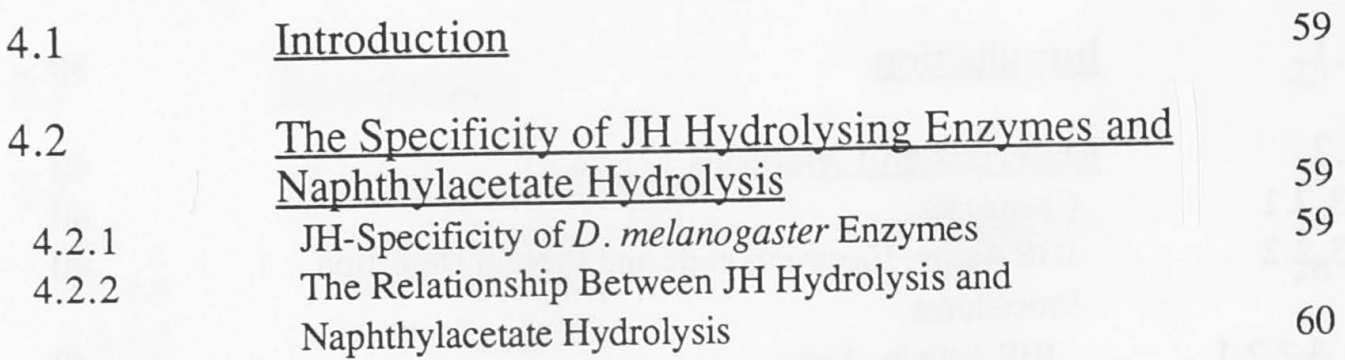

4.3

4.3.2

4.3.2.1

4.3.2.2

4.3.2.3

4.3.2.4

4.3 .3

4.3.4

4.4

4.4.1

4.4.2

4.4.2.1

4.4 .2 .2

4.4 .2 .3

4.4.2.4

4.5

$\underline{\text { Structure and Regulation of JHE }}$

4.6

Conclusions 


\section{Summary}

Juvenile hormone $(\mathrm{JH})$ is one of the main morphogenic hormones of insects and degradation of the hormone is believed to play an important role in its regulation in many insects. The two main routes for $\mathrm{JH}$ degradation are hydroysis by JH esterase (JHE) and JH epoxide hydrolase (JHEH). A major focus of research has been on juvenile hormone esterase (JHE) found in the haemolymph of final instar lepidopteran larvae, although considerable hydrolysis of $\mathrm{JH}$ occurs in other tissues and at other life stages. In contrast, no comprehensive study of JH degradation has been undertaken in any dipteran species. However, the available evidence suggests that very little JH degrading activity is found in the haemolymph of Diptera and several dipteran JHEs exhibit atypical patterns of sensitivity to inhibitors. These data suggested that both the expression pattern and the biochemical properties of dipteran $\mathrm{JH}$ degrading enzymes may differ from other insect orders, and therefore, that the role of JH degradation may be different.

The aim of this project was to comprehensively characterise $\mathrm{JH}$ degradation in the dipteran, Drosophila melanogaster, with special emphasis on JHE. The first objective was to identify the $\mathrm{JH}$ degrading enzymes of $D$. melanogaster and the second was to determine their expression patterns and biochemical properties in order to determine their role in the regulation of $\mathrm{JH}$ titre. These data were compared with other data to determine whether a general model could be developed for dipteran JH titre regulation. Finally, the roles of JH hydrolysis in the regulation of $\mathrm{JH}$ titre were compared among Diptera, Lepidoptera and other insects.

Two approaches were taken to identify the JH degrading enzymes of $D$. melanogaster. The first was to investigate the properties of an esterase, denoted EST20, which can be visualised after native polyacrylamide gel electrophoresis using artificial esterase substrates, $\alpha$ - and $\beta$-naphthylacetate. EST20 is shown to be a likely homologue of the p-esterase of Drosophila virilis, which has been claimed to be the JHE of this species. However, this study shows that $\mathrm{JH}$ hydrolytic activity is associated with neither EST20 in D. melanogaster nor the p-esterase in $D$. virilis.

The second approach was to use a radiometric assay which identified two $\mathrm{JH}$ hydrolysing enzymes, JHE and JHEH. The developmental profile of JH hydrolytic activity in $D$. melanogaster correlates inversely with profiles of $\mathrm{JH}$ titre and production. Key features are prewandering and prepupal maxima of JH hydrolytic activity in whole body homogenates of final instar larvae. This developmental profile of expression is similar to the lepidopteran pattern suggesting that the role of JH hydrolysis is essentially similar during this period. In D. melanogaster, the prewandering maximum consists mainly of JHEH whereas the prepupal peak consists mainly of JHE. However, the relative contributions of JHE and JHEH at these times differs both among the Diptera and in other orders.

JHE from the prepupal stage was purified to homogeneity by selective precipitations, isoelectric focussing, anion exchange and gel filtration chromatography, and its $\mathrm{N}$-terminal sequence was determined. Its $\mathrm{K}_{\mathrm{M}}$ and $\mathrm{V}_{\max }$ for JHIII hydrolysis are $89 \mathrm{nM}$ and $0.59 \mu \mathrm{mol} / \mathrm{min} / \mathrm{mg}$, respectively, indicating a specificity constant of $6.8 \times 10^{6} \mathrm{M}^{-1} \mathrm{sec}^{-1}$. JHE also hydrolyses $\alpha$-naphthylacetate with a $\mathrm{K}_{\mathrm{M}}$ of $120 \mu \mathrm{M}$ and a specificity constant of $5.9 \times 10^{5} \mathrm{M}^{-1} \mathrm{sec}^{-1}, 12$ fold lower than for JHIII. 
Two forms of juvenile hormone, JHIII and JHIIIbisepoxide $\left(\mathrm{JHB}_{3}\right)$ may have roles in the higher Diptera. Further kinetic experiments showed that binding in the active site of JHE is promoted by the features found in $\mathrm{JHII}$ and $\mathrm{JHB}_{3}$ and reduced by almost any departure from these, suggesting that both molecules are in vivo substrates. Studies of the interaction of JHE with the JH carrier, lipophorin, indicate that competition with lipophorin for binding of $\mathrm{JH}$ may prevent $\mathrm{JH}$ hydrolysis at some life stages. However, lipophorin may assist in the clearance of JH from the organism at other times.

The developmental profiles of both JHE and JHEH expression and the kinetic properties of JHE indicate that $\mathrm{JH}$ is the in vivo substrate for these enzymes and that $\mathrm{JH}$ degradation plays an important role in the regulation of JH titre in $D$. melanogaster. Comparison with data from the literature enabled the development of a generalised model for the expression pattern and role of $\mathrm{JH}$ degradation in the Diptera. The model indicates that the expression pattern and role of $\mathrm{JH}$ degradation in the Diptera is more similar to the well characterised lepidopteran system than was previously apparent. Finally, the roles of the dipteran $\mathrm{JH}$ isoforms, JHIII and $\mathrm{JHB}_{3}$ are considered. It is proposed that the $\mathrm{JH}$ isoforms may be differentially regulated by a combination of differential synthesis and differential hydrolysis, and that hydrolysis may be modulated by differential binding of $\mathrm{JH}$ isoforms to lipophorin. 


\section{Chapter 1}

\section{Introduction and Literature Review}

\subsection{Introduction}

Juvenile hormone $(\mathrm{JH})$ is one of several important insect hormones whose interplay regulates development and reproduction (Gupta, 1990a; Riddiford, in press, for reviews). JH has been described as the "inhibitory" or "status quo" hormone of arthropods and is proposed to modify the action of moulting hormone (ecdysteroids, Willis, 1981). Thus, it is believed to inhibit progress from one developmental program to the next, such as from a larval program to a pupal one or from a pupal to an adult program (Willis, 1990; Tata, 1993). The JH titre is therefore critical for regulation of developmental events. However, the role of JH in larval Diptera remains conjectural, partly because $\mathrm{JH}$ has different effects and sensitive periods in dipteran larvae compared with other species (Richard and Gilbert, 1991; Riddiford, in press).

At certain times in development a reduced $\mathrm{JH}$ titre is required and in many insects both reduced synthesis of $\mathrm{JH}$ and increased $\mathrm{JH}$ degradation are important to affect this change (de Kort and Granger, 1981; Hammock, 1985; Roe and Venkatesh, 1990, for reviews). Temporally and spatially regulated $\mathrm{JH}$ degrading enzymes are most thoroughly characterised in the Lepidoptera, with juvenile hormone esterase (JHE) studied most intensively. In contrast, the dipteran $\mathrm{JH}$ degrading enzymes are poorly characterised, although the available evidence suggests that the patterns of expression and properties of dipteran JH degrading enzymes differ markedly from other insects. These observations have lead to the speculation that the roles of JHE may differ between Lepidoptera and Diptera.

The aim of my project was to comprehensively characterise JH degradation in a dipteran and compare and contrast the physiological and biochemical properties of the $\mathrm{JH}$ degrading enzymes in Diptera with those in Lepidoptera, with special emphasis on JHE. Having defined the dipteran JH degrading enzymes a further aim was to assess the relative contributions of degradation to regulation of JH titre between Diptera and Lepidoptera.

This chapter reviews the relevant literature. The roles of $\mathrm{JH}$ are discussed with particular emphasis on its regulation of metamorphosis. Mechanisms of JH titre regulation via changes in synthesis, interactions with $\mathrm{JH}$ carrier proteins and activity of degrading enzymes are reviewed. The degradation of $\mathrm{JH}$ is reviewed in greater detail, reflecting the emphasis of this study.

\subsection{The Structures and Distribution of Juvenile Hormones}

Juvenile hormones are closely similar sesquiterpenoid compounds produced by insects. The most common isoform is JHIII (methyl (2E,6E)-10,11-epoxy-3,7,11trimethyl 2,6-dodecadienoate) which is found in all of the nine insect orders investigated (Schooley et al., 1984). Other isoforms of $\mathrm{JH}$ are very similar to this molecule, 
differing slightly by the substituents on the acid moiety (Fig. 1.1). All are esters of methanol, have a 10(R),11 epoxide group, and a 2,3 double bond. The 2,3 double bond is conjugated with the ester group leading to increased resistance of the ester to hydrolysis.

The Lepidoptera is the only order in which four isoforms of $\mathrm{JH}(\mathrm{JH} 0, \mathrm{JHI}$, JHII, and 4-methyl JHI) have been identified reliably (Schooley et al., 1984), with the exception of one report of JHI in an Hemipteran (Numata et al., 1992). JH0, JHI, and JHII differ from JHII by the replacement of one or more of the methyl side-chains of JHIII with ethyl side-chains and 4-methyl JHI has an additional methyl side chain.

Key aspects of the JH system may be common to all arthropods (Cusson et al., 1991). It has been proposed that methylfarnesoate (MF), which differs from JHIII only by the lack of the 10,11 epoxide group, or a similar molecule is the ancestral form of JH. MF is found in Crustacia (Gupta, 1990b; Cusson et al., 1991) and its titre varies with size and reproductive status through development in a manner which suggests that MF has functions similar to JH in insects (Borst and Tsukimra, 1992; Laufer et al., 1993). In the Myriapoda there is evidence, suggesting the presence of JH-like compounds but none have been isolated (Gupta, 1990b). Unidentified JH-like compounds are produced by Chelicerata which are probably similar to insect JHs because insect $\mathrm{JHs}$ and agonists have $\mathrm{JH}$-like and anti-JH-like effects, respectively, in several species (Bonaric and Juberthie, 1990), and developmentally regulated JHE and juvenile hormone epoxide hydrolase (JHEH) activities are found in one chelicerid (Roe and Venkatesh, 1990). In addition, it is possible that JH-like compounds will be found outside the arthropod group because ecdysteroids are found in annelids and nematodes and these hormones may have evolved together with a JH-like endogenous control mechanism (Gupta, 1990b).

JHIII bisepoxide $\left(\mathrm{JHB}_{3}\right)$ has been found only in higher Diptera and differs from JHIII by epoxidation of the 6,7 double bond (Richard et al., 1989b; Hearlt et al., 1993). Both $D$. melanogaster and Calliphora vomitoria produce JHIII as a minor product and $\mathrm{JHB}_{3}$ as the major product, whereas $\mathrm{JHB}_{3}$ is reported to be the only $\mathrm{JH}$ isoform produced in Lucilia cuprina (LeFevere et al., 1993). There is some dispute about the identification of the JHIII product and the available evidence on the functional roles of $\mathrm{JHIII}$ and $\mathrm{JHB}_{3}$ does not resolve whether both isoforms are physiologically relevant in the higher Diptera. Evidence that supports a role for JHIII includes the similarity of developmental changes of its titre in Drosophila hydei and D. melanogaster with those in other insects (Bührlen et al., 1984; Bownes and Rembold, 1987; Sliter et al., 1987). In addition, the $\mathrm{JH}$ carrier in D. melanogaster haemolymph has a greater binding affinity for JHIII than any other JH carrier (Shemshedini and Wilson, 1988). The $L$. cuprina JH carrier also has a high affinity for JHIII, with preliminary results suggesting a low affinity for $\mathrm{JHB}_{3}$ (Dr. S. C. Trowell, pers. comm.). However, in D. melanogaster adults, $\mathrm{JHB}_{3}$ production is temporally regulated while JHIII and MF are produced at relatively constant rates, suggesting that the latter may merely be precursors for $\mathrm{JHB}_{3}$ (Altaratz et al., 1991).

In D. melanogaster, different bioassays provide examples of both greater sensitivity to JHIII over $\mathrm{JHB}_{3}$ and vice versa (Saunders et al., 1990; Richard et al., 1989b; Section 4.4.1), perhaps indicating different, but specific roles for both JHIII and $\mathrm{JHB}_{3}$. However, non-physiological $\mathrm{JH}$ isoforms are sometimes more potent than physiological isoforms in bioassays, possibly due to less rapid degradation of the nonphysiological isoform (Richard et al., 1989b). Investigations of the metabolism of 
<smiles>COC(=O)/C=C(\C)CC/C=C(\C)CCC=C(C)C</smiles><smiles>CC/C(=C\C(=O)OC)CC/C=C(\CC)CCC1OC1(C)CC</smiles><smiles>CCC(=CCCC(C)=CC(=O)OC)CCC1OC1(C)CC</smiles><smiles>CCC(=CCC(C)C(C)=CC(=O)OC)CCC1OC1(C)CC</smiles><smiles>CCC1(C)OC1CC/C(C)=C/CC/C(C)=C/C(=O)OC</smiles><smiles>COC(=O)/C=C(\C)CC/C=C(\C)CCC1OC1(C)C</smiles><smiles>COC(=O)/C=C(\C)CCC1OC1(C)CCC1OC1(C)C</smiles><smiles>CCC(=O)Oc1cccc2ccccc12</smiles><smiles>CCC(=O)Oc1ccc2ccccc2c1</smiles>

Methylfarnesoate

JHO

JHI

4-Me JHI

JHII

JHIII

$\mathrm{JHB}_{3}$

$\alpha$-Naphthylacetate

B-Naphthylacetate

Figure 1.1 JH Isoforms and the Artificial Esterase Substrates $\alpha$ - and $\beta$-Naphthylacetate 
JHIII and $\mathrm{JHB}_{3}$ suggest the presence of more than one JHEH with different specificities for $\mathrm{JHIII}$ and $\mathrm{JHB}_{3}$. In $D$. melanogaster larvae, cell fractions differ in their relative rates of epoxide hydrolysis of JHIII and $\mathrm{JHB}_{3}$, although JHIII is more rapidly hydrolysed in all fractions (Casas et al., 1991; Harshman et al., 1991). These findings suggest that $\mathrm{JHIII}$ and $\mathrm{JHB}_{3}$ may be regulated differentially by spatially or temporally specific degradation.

\subsection{Functions of $\mathrm{JH}$}

$\mathrm{JH}$ has a variety of functions that can be broadly divided into two classes associated with reproduction or development. In the first class, $\mathrm{JH}$ has reproductive roles in both males and females (Koeppe et al., 1985, for review). The second class encompasses developmental arrest (diapause) (Denlinger and Tanaka, 1989; de Kort, 1990) and metamorphosis, of which caste and morph determination (Hartfelder et al., 1993; Zera and Holtmeier, 1992) could be considered special cases.

Some of these functions were determined experimentally prior to the isolation of $\mathrm{JH}$ and other hormones. Such experiments included ligation between body parts to prevent the circulation of hormones to all parts of the insect, transplantation of putative hormone-producing organs and hormone-responsive tissues, and the joining of different live insects or parts of insects at different developmental stages (Wigglesworth, 1954, for review). Later work used $\mathrm{JH}$ applications and inhibitors of $\mathrm{JH}$ production to further characterise its roles.

The best characterised reproductive role of $\mathrm{JH}$ is its regulation of vitellogenesis (egg production) in some insects including Diptera, Lepidoptera, and Blattodea (Tobe et al., 1985; Borovsky et al., 1992; Satyanarayana et al., 1992), although it also regulates the development of both male and female gonadotrophic organs (Yamomoto et al., 1988; Kelly et al., 1987), and sex determination in aphids (Hales and Mittler, 1988). In adult female Diptera, $\mathrm{JH}$, ecdysone and dietary factors act in combination to stimulate ovarian maturation and vitellogenin (the precursor of vitellin, the major insect yolk protein) synthesis and uptake by oocytes (Kelly et al., 1987; Adams and Gerst, 1992). In $D$. melanogaster, several lines of evidence indicate that $\mathrm{JH}$ regulates vitellogenesis and ovarian diapause. In the apterous mutant of $D$. melanogaster, $\mathrm{JHB}_{3}$ production remains at the level found at eclosion, JHII titre is lower than in wild type flies, and the vitellogenic cycle is blocked at an early stage (Bownes, 1989; Altaratz et al., 1991). Ovarian diapause (in which yolk proteins are not deposited in the oocytes) is reversed by topical application of $\mathrm{JH}$ and corpora allata removed from non-diapausing flies exhibit higher rates of in vitro $\mathrm{JHB}_{3}$ production (Saunders et al., 1990).

The metamorphic role of $\mathrm{JH}$ has been stated generally as maintenance of the current developmental state of the insect. Its removal and/or non-production prior to the pupal and adult moults permits redifferentiation in response to the signal to moult (Willis, 1981; Kumaran, 1990). Programs of gene expression specific for each life stage and cell type are determined during the embryonic stage. Thus, in holometabolous larvae, ecdysone with low JH causes a moult during which cells change from their larval programs of gene expression to their pupal programs of gene expression. Similarly, in pupae, ecdysone with low JH causes a moult during which cells change from their pupal programs of gene expression to their adult programs of gene expression. The presence of $\mathrm{JH}$ at a moult prevents progress to the next program of 
gene expression, but the absence of JH permits progress. Regulation of metamorphosis is the best characterised role of $\mathrm{JH}$ and is discussed in greater detail below.

\subsection{Physiology and Hormonal Regulation of Insect Metamorphosis}

\subsubsection{Physiology of Metamorphosis}

In order for arthropods to grow, periodic shedding of old cuticle (moulting) must occur. In the holometabolous insects, which includes the Diptera and Lepidoptera, a series of larval stages or instars are separated by moults (three for the DipteraCyclorrapha (higher flies); generally around five for the Lepidoptera), followed by a moult to the pupal form, and then a moult to the adult form. The final larval instar is divided into a feeding phase and a post-feeding or "wandering" phase, during which larvae seek a suitable site to undergo the pupal moult. In the cyclorraphous Diptera the pupal and adult moults occur within the puparium, a unique hard case which is formed by contraction and tanning of the cuticle of the final larval instar (pupariation). A "prepupal" phase, which should be regarded as a continuation of the larval stage (Roberts and Gilbert, 1986), commences with pupariation and ends when the epidermis separates from the puparium (pupal apolysis), about twelve hours later in D. melanogaster. Pupal apolysis is regarded as the equivalent of the pupal moult in other insects even though the pupa does not break out of the larval cuticle until much later. The pupa to adult moult (adult apolysis) occurs when the pupal cuticle separates from the underlying epidermis but the "pharate adult" does not immediately break out of the puparium (eclosion). In D. melanogaster, adult apolysis occurs about 34-50 hours after pupariation and eclosion occurs about 100 hours after pupariation (Fraenkel and Bhaskaran, 1973; Bainbridge and Bownes, 1981).

In holometabolous insects, almost all larval tissues are replaced after metamorphosis by pupal then adult tissues (Wigglesworth, 1954; Madhavan and Schneiderman, 1977). Those adult tissues which have larval homologues can be derived either from larval cells or imaginal (adult) anlage (set aside) cells. Homologous tissues in adult insects whose larvae do not grow rapidly, such as Coleoptera and Lepidoptera, are derived from larval cells. That is, the cells which formed larval tissues redifferentiate under the influence of an altered hormonal environment to form the adult tissues. However, in insects whose larvae grow rapidly such as Diptera, many larval cells hypertrophy, so a separate stock of diploid anlage cells are set aside during embryogenesis for redifferentiation. These are found scattered, in small clusters or as rings within various tissues such as the gut, trachea and abdominal epidermis. At metamorphosis the anlage cells grow, proliferate, differentiate and replace the larval cells of these tissues (Pearson, 1972; Madhavan and Schneiderman, 1977).

The specialised, adult structures which have no larval homologues such as eyes, wings, legs and antennae are derived from a special class of imaginal cells, the imaginal disc cells. Growth and differentiation of these cells occurs during the larval stage by invagination into the lumen of the imaginal discs. During metamorphosis the imaginal discs are incorporated into the complete pupa with evagination and growth of the specialised structures (Madhavan and Schneiderman, 1977). 
Thus, except for the specialised structures, the pupal and adult cuticles of lepidopterans are secreted by the same epidermal cells as produced the larval cuticle. In contrast, the entire adult cuticle of dipterans is secreted by different cells from the larval cuticle (Pearson, 1972; Roseland and Schneiderman, 1979). The adult abdominal epidermis is derived from abdominal anlage cells ("abdominal histoblasts"), and unlike other insects, the imaginal discs provide the cells of the general epidermis of the adult head and thorax in addition to the specialised adult structures.

In D. melanogaster, the imaginal discs and abdominal histoblasts differ by both the time at which they recommence cell division after embryogenesis and the time at which they replace larval tissues (Madhavan and Schneiderman, 1977). Imaginal discs recommence cell division during the first or second instar, are incorporated into the head and thorax during the larva to pupa metamorphosis, and secrete first a pupal then an adult cuticle. In contrast, the abdominal histoblasts do not divide between the emergence of the larva from the egg and five hours after pupariation. The abdominal histoblasts then spread out to cover the abdomen just after head eversion (larva-pupa apolysis), replace the larval epidermis, and then secrete the adult abdominal cuticle (Madhavan and Schneiderman, 1977; Roseland and Schneiderman, 1979). Thus, the pupal cuticle of the head and thorax is largely secreted by imaginal disc-derived cells but the pupal cuticle of the abdomen is secreted by the larval epidermal cells. Unlike D. melanogaster, the abdominal histoblasts of other higher Diptera such as Dacus tryoni and Calliphora erythrocephala recommence cell division during the larval stage along with the other imaginal cells (Anderson, 1964; Pearson, 1972).

\subsubsection{Hormonal Regulation of Metamorphosis}

A generalised scheme for the regulation of metamorphosis in holometabolous insects is presented below (Roe and Venkatesh, 1990; Riddiford, in press, for reviews). All moults are initiated by ecdysone, JH determines the outcome of the moult and several other hormones also have roles. Release of the moult-initiating pulse of ecdysone from the prothoracic glands is triggered by the release of prothoracicotrophic hormone (PTTH) from a set of neurosecretory cells in the brain. Ecdysone is then converted to more active metabolites, ecdysteroids, in the fat body and various target tissues. An early event in the moulting process is the separation of the old cuticle from the epidermis (apolysis). Moulting concludes with the shedding of the old cuticle (ecdysis) caused by eclosion hormone, a peptide released from other neurosecretory cells when the ecdysteroid titre has fallen to a low level.

However, during the final instar, at least one pulse of ecdysone occurs which does not immediately cause a moult but is involved in the commitment to metamorphosis. After the final larval moult JH titres decline as a result of decreased synthesis and increased degradation. PTTH is released in response to very low JH titre and other physiological or environmental cues (critical weight or critical ratio of body dimensions in some Lepidoptera, Jones et al., 1981), causing a small pulse of ecdysone release (Watson et al., 1987). In both Lepidoptera and Diptera this is a major endocrine event with major changes in gene expression (Cherbas, 1993) and in the types of RNA synthesised (Shaaya, 1993). The ecdysteroids, together with low JH titre, cause commitment to the production of pupal rather than larval proteins at the next ecdysone pulse, as well as a behavioural change from feeding to wandering (Dominick and Truman, 1985). 
Pupation is initiated by the next pulse of ecdysone which coincides with a pulse of $\mathrm{JH}$ production in Lepidoptera (Baker et al., 1987) and D. melanogaster (Richard et al., 1989a). In Manduca sexta, this JH pulse prevents adult differentiation of the imaginal discs during the pupal moult (Kiguchi and Riddiford, 1978). Pupariation occurs at this time in the higher Diptera. The JH titre immediately falls again, before the pupal moult occurs, and in Lepidoptera this is due to decreased synthesis and increased degradation (Roe and Venkatesh, 1990). In D. melanogaster a pulse of ecdysone occurs about twelve hours after pupariation, coinciding with pupal apolysis. During the pupal stage there is an ecdysone pulse without $\mathrm{JH}$, which causes the adult moult and stimulates the expression of adult-specific genes (Riddiford, in press).

Although the available information suggests that the patterns of $\mathrm{JH}$ and ecdysone titre changes in D. melanogaster are similar to those found in the Lepidoptera (Bownes and Rembold, 1987; Sliter et al., 1987; Richard et al., 1989a; Altaratz et al., 1991; Dai and Gilbert, 1991; Riddiford, in press; Fig. 1.2), two lines of evidence indicate that the commitment to pupation in Diptera occurs at an earlier stage than for insects such as the Lepidoptera. Firstly, Lepidoptera and other insects can be induced to undergo supernumary larval moults by the application of $\mathrm{JH}$ in the final instar. In contrast, $\mathrm{JH}$ application to final instar dipterans delays pupariation, which can be rescued with ecdysone injection (Srivastava and Gilbert, 1969), but supernumary larval moults cannot be induced (Sehnal and Zdarek, 1976). Secondly, starved, final instar larvae of Diptera pupate in concert with fed larvae and become undersized but fertile adults (Bakker, 1959; Srivastava and Gilbert, 1969), unlike starved Lepidoptera which experience delayed pupation or supernumary larval moults (Cymborowski et al., 1982).

The time at which different $D$. melanogaster tissues are sensitive to $\mathrm{JH}$ treatment varies. Exposure to high levels of $\mathrm{JH}$ analogues in the first and early second instar, but not later, prevents the adult development of the head and thorax (Riddiford and Ashburner, 1991). In contrast, JH and JH analogues applied to late larvae or prepupae do not affect pupariation but severely inhibit the adult development of the abdomen (Bhaskaran, 1972; Postlethwait, 1974). When JH is applied to prepupae of D. melanogaster most of the normal proliferation and redistribution of abdominal histoblast cells occurs (Dr. L. M. Riddiford, pers. comm.), yet these cells produce an abnormal cuticle which lacks bristles and resembles a second larval or pupal cuticle. However, it remains to be determined whether the profile of proteins secreted by the epidermal cells most closely resembles a larval, pupal or adult pattern. In summary, the period of sensitivity of tissues to $\mathrm{JH}$ coincides with the commencement of postembryonic cell division in the imaginal cells from which the tissues are derived (Section 1.4.1, Riddiford and Ashburner, 1991).

The ability to induce supernumary larvae in the Lepidoptera but not the Diptera appears to be a consequence of their different mode of metamorphosis. Lepidoptera largely rely on redifferentiation of larval cells at the pupal moult and this redifferentiation can be inhibited by JH. In contrast, Diptera replace at least the epidermal cells of the head and thorax at this moult (Section 1.4.1) and it appears that the commitment to this process is made much earlier in larval development. At the the pupa to adult moult, the anti-metamorphic effect of $\mathrm{JH}$ on dipteran pupae is typical of other insects, with the earliest pupal applications of $\mathrm{JH}$ causing retention of pupal characteristics in the greatest number of structures (Srivastava and Gilbert, 1969). 
Figure 1.2 Comparison of the Ecdysteroid Titre, JH Titre and JH Production During Development of D. melanogaster

(A) shows the titre of ecdysteroids during development. The figure is reproduced from Riddiford (in press) and the data were compiled from ten sources cited therein.

(B) shows the titre of JHIII during development. Solid bars indicates male titres and open bars indicate female titres. The figure is reproduced from Riddiford (in press) and the data were compiled from Bownes and Rembold (1987) and Sliter et al. (1987).

(C) shows in vitro $\mathrm{JH}$ production by brain/ring gland/ventral ganglion complexes or adult corpora allata aligned with the profiles of ecdysteroid and JHIII titres. Solid symbols indicate JHB 3 production and open symbols indicate JHIII production. The data were compiled from Richard et al. (1989a), Altaratz et al. (1991) and Dai and Gilbert (1991). Pupal age is indicated from pupariation although the larva/pupa transition occurs at pupation (pupal apolysis), twelve hours later (Section 1.4.1). 

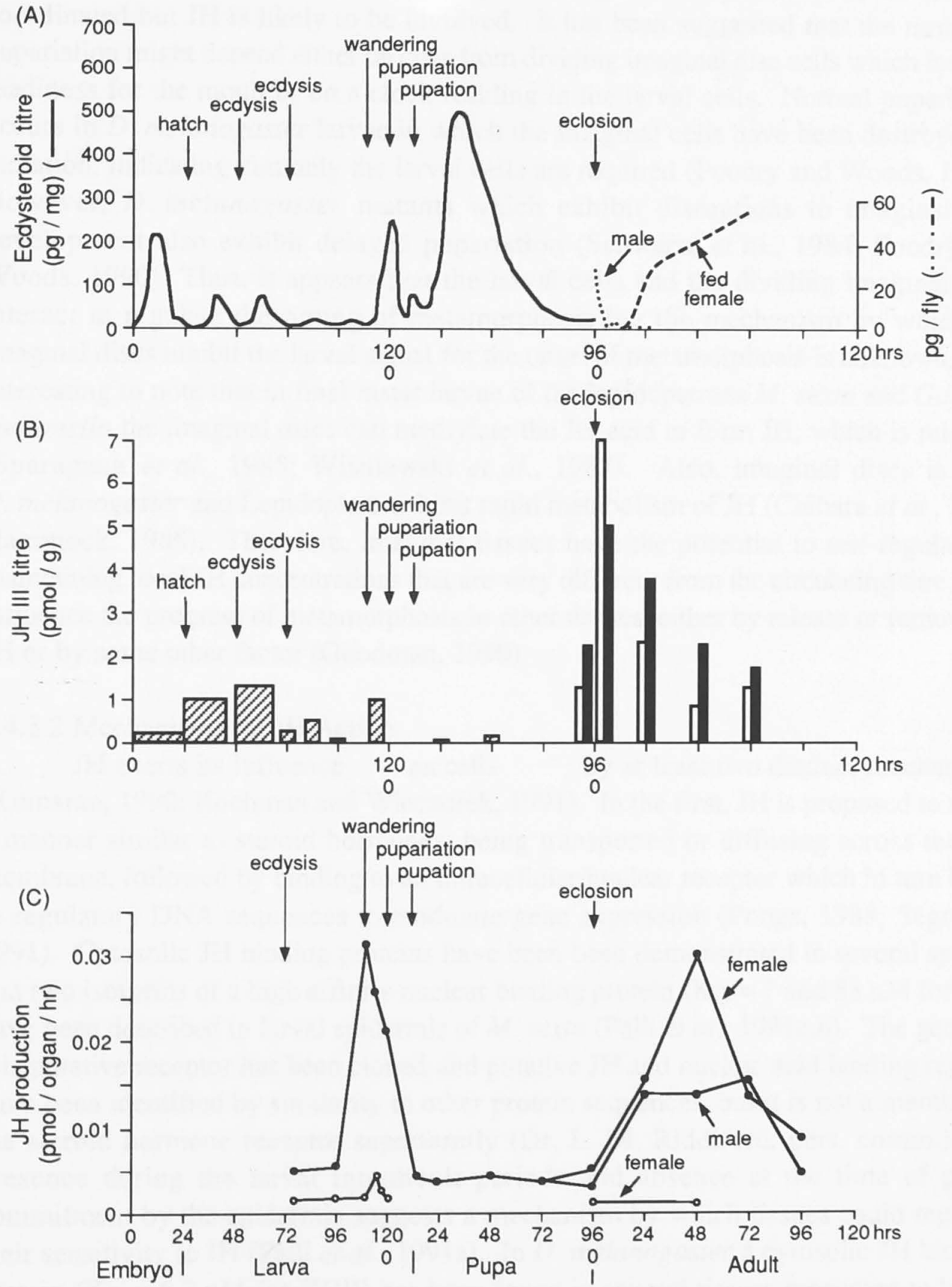


\subsubsection{Mechanisms Underlying Hormonal Control of Metamorphosis}

\subsubsection{Timing of Metamorphosis}

It is not known how the metamorphic changes of imaginal and larval cells are coordinated but $\mathrm{JH}$ is likely to be involved. It has been suggested that the timing of pupariation might depend either on cues from dividing imaginal disc cells which indicate readiness for the moult or on a clock residing in the larval cells. Normal pupariation occurs in $D$. melanogaster larvae in which the imaginal cells have been destroyed by radiation, indicating that only the larval cells are required (Poodry and Woods, 1990). However, D. melanogaster mutants which exhibit disruptions to imaginal disc development also exhibit delayed pupariation (Schwartz et al., 1984; Poodry and Woods, 1990) Thus, it appears that the larval cells and the dividing imaginal cells interact to regulate the timing of metamorphosis but the mechanism by which the imaginal discs inhibit the larval signal for the onset of metamorphosis is unknown. It is interesting to note that in final instar larvae of the lepidopterans $M$. sexta and Galleria mellonella the imaginal discs can methylate the $\mathrm{JH}$-acid to form $\mathrm{JH}$, which is released (Sparagana et al., 1985; Wisniewski et al., 1987). Also, imaginal discs in both D. melanogaster and Lepidoptera exhibit rapid metabolism of JH (Chihara et al., 1972; Hammock, 1985). Therefore, imaginal tissues have the potential to self-regulate by maintaining local $\mathrm{JH}$ concentrations that are very different from the circulating titre, or to influence the progress of metamorphosis in other tissues, either by release or removal of $\mathrm{JH}$ or by some other factor (Goodman, 1990).

\subsubsection{Mechanisms for JH Action}

JH exerts its influence on cells by at least two distinct mechanisms (Kumaran, 1990; Kochman and Wieczorek, 1991). In the first, JH is proposed to act in a manner similar to steroid hormones, being transported or diffusing across the cell membrane, followed by binding to an intracellular/nuclear receptor which in turn binds to regulatory DNA sequences to modulate gene expression (Pongs, 1988; Segraves, 1991). Cytosolic JH binding proteins have been been demonstrated in several species and two isoforms of a high affinity nuclear binding protein $\left(\mathrm{K}_{\mathrm{D}}=7\right.$ and $88 \mathrm{nM}$ for JHI) have been described in larval epidermis of M. sexta (Palli et al., 1991a,b). The gene for this putative receptor has been cloned and putative $\mathrm{JH}$ and nucleic acid binding regions have been identified by similarity to other protein sequences, but it is not a member of the steroid hormone receptor superfamily (Dr. L. M. Riddiford, pers. comm.). Its presence during the larval intermoult periods and absence at the time of pupal commitment by the epidermis suggests a mechanism by which tissues could regulate their sensitivity to JH (Palli et al., 1991a). In D. melanogaster a cytosolic JH binding protein $\left(\mathrm{K}_{\mathrm{D}}=6.7 \mathrm{nM}\right.$ for $\left.\mathrm{JHIII}\right)$ has been found in several tissues presumed to be $\mathrm{JH}$ sensitive and not in tissues presumed to be insensitive (Shemshedini et al., 1990). Furthermore, the binding affinity of the protein for JHIII is 10 -fold higher in strains which are susceptible to the $\mathrm{JH}$ analogue, methoprene than in mutants exhibiting resistance to methoprene, strongly suggesting that the binding protein is the $\mathrm{JH}$ receptor (Shemshedini and Wilson, 1990).

The second mechanism by which $\mathrm{JH}$ appears to exert its influence on cells appears to be mediated by membrane protein/second messenger systems and involves activation of protein kinase C (Yamomoto et al., 1988; Sevala and Davey, 1989). However, the presumptive intracellular receptor was also found in one of these tissues, 
suggesting that both mechanisms of response to $\mathrm{JH}$ may operate in a single tissue (Shemshedini et al., 1990).

A third mechanism by which $\mathrm{JH}$ may exert its effects is by modulation of polyamine levels, but this proposal remains highly conjectoral. The anti-metamorphic effect of $\mathrm{JH}$ may be a consequence of a block to redifferentiation caused by a block to chromatin structural changes (Willis, 1981, 1990). This effect has been proposed to be mediated by polyamines, which in vertebrates regulate DNA, RNA and protein synthesis, modulate the phosphorylation of specific proteins, and are essential for cell growth and differentiation (Strambi et al., 1993). Although few studies have been performed in insects, the following examples indicate that polyamines are likely to play key, albeit poorly understood, roles in the response of insect cells to ecdysone and $\mathrm{JH}$. In both a bovine system and D. melanogaster, $\mathrm{JH}$ can modulate the induction of ornithine decarboxylase (ODC), the first key enzyme in the biosynthesis of polyamines (Birnbaum and Gilbert, 1990), and in the house cricket Acheta domesticus, both ecdysone and JH modulate polyamine synthesis, ODC activity and phosphorylation of specific proteins (Strambi et al., 1992, 1993). Polyamine levels peak in Tenebrio molitor epidermis just before pupal ecdysis between the last larval ecdysone peak and a period of JH sensitivity (Besson et al., 1986), and in $M$. sexta fat body and neural tissue fluctuations in polyamine concentrations and ODC activity appear to be associated with periods of growth, differentiation and proliferation during metamorphosis (Birnbaum et al., 1988).

\subsubsection{Mechanisms for Ecdysone Action}

In $D$. melanogaster regulation of gene expression by ecdysteroids has been extensively studied, together with the effect of $\mathrm{JH}$ on this expression. The temporally regulated "puffs" in the giant polytene chromosomes of the salivary glands of third instar larvae and early pupae have served as a model for ecdysone-induced changes (Andres and Thummel, 1992, for review). A well defined sequence of early and late puffs occur in response to three pulses (pre-wandering, pupariation and pupal apolysis) of ecdysone and these puffs correspond to the activation of genes. The early gene products include proteins whose sequence includes DNA binding domains, supporting the proposal that these are gene regulators which turn on the late genes, while possibly also inhibiting their own production. This model is further supported by the finding that antibodies to one of the early gene products binds to many of the early and late ecdysone-induced puffs (Andres and Thummel, 1992).

The puffing sequence can be reproduced in vitro by appropriately timed incubations with ecdysone-containing and ecdysone-free media. JH has been shown to modify the competence of salivary glands to respond to ecdysone (Richards, 1978), indicating that many of the genes regulated by ecdysone are also regulated by $\mathrm{JH}$ (and presumably by tissue specific factors). The prepupal ecdysone-induced puffing sequence can be inhibited by $\mathrm{JH}$ with the $\mathrm{JH}$ sensitive period occurring prior to prepupal ecdysone exposure. However, the late larval puffing sequence is not influenced by JH. The coincidence of the JH sensitive periods for salivary gland chromosomal puffs and adult abdominal defects (Postlethwait, 1974) suggests that the prepupal period is generally important for expression of $\mathrm{JH}$ regulated genes. 


\subsection{Regulation of JH Titre}

In order to regulate the variety of processes described above, the abundance of $\mathrm{JH}$ itself must be tightly regulated. Regulation of $\mathrm{JH}$ titre is achieved by a balance between synthesis and degradation. However, JH carrier molecules can modulate these two processes, and other mechanisms such as sequestration and excretion may also contribute to modulation of the JH titre (de Kort and Granger, 1981; Hammock, 1985; Tobe and Stay, 1985; Roe and Venkatesh, 1990, for reviews).

\subsubsection{Synthesis of JH}

$\mathrm{JH}$ is produced in a specialised organ, the corpus allatum. In dipteran larvae, however, the corpus allatum is part of a composite organ, the ring gland, which includes the prothoracic gland and the corpus cardiacum. Although the corpus allatum and regulation of JH synthesis have been studied in many insects (Tobe and Stay, 1985; Goodman, 1990, for reviews), JH synthesis in Diptera has only recently received detailed attention (Richard et al., 1989a,b, 1990; Richard and Gilbert, 1991; Altaratz et al., 1991; Dai and Gilbert, 1991; Duve et al., 1992).

Regulation of JH synthesis by the nervous system has been demonstrated in many insect orders, mediated by the products of neurosecretory cells and possibly conventional electrical impulses. Regulation occurs both at several early steps in its biosynthetic pathway and at the terminal steps (Goodman, 1990; Borovsky et al., 1992). Terminal steps in the JH biosynthetic pathway involve the esterification of farnesoate to MF and 10,11 epoxidation (and 6,7 epoxidation for $\mathrm{JHB}_{3}$ ), although the order of these steps appears to vary between species (Tobe and Stay, 1985). In D. melanogaster, JH production appears to be regulated both by the availability of JH precursors and the activity of the oxidase(s) which adds the epoxide group(s). Feedback inhibition of $\mathrm{JH}$ production by $\mathrm{JH}$ occurs at least at two levels. $\mathrm{JHB}_{3}$ stimulates the brain to produce an allatostatic factor which is transfered to the corpus allatum via nervous connections (Altaratz et al., 1991), but JHIII and $\mathrm{JHB}_{3}$ also inhibit $\mathrm{JH}$ production by ring glands which are isolated from the brain (Richard and Gilbert, 1991).

Temporal regulation of $\mathrm{JH}$ production is one of the major factors causing variation of $\mathrm{JH}$ titre (Goodman, 1990). In D. melanogaster, regulation of JH production has been investigated in final instar larvae and adults (Fig. 1.2). In late wandering larvae a peak of JH synthesis occurs which declines abruptly one or several hours after pupariation (Richard et al., 1989a; Dai and Gilbert, 1991) and in adults JH synthesis rises then falls during the first four days after eclosion (Altaratz et al., 1991; Dai and Gilbert, 1991). The latter peak in females coincides with the onset of vitellogenesis, histolysis of the larval fat body and changes in sexual behaviour. These two periods of JH synthesis coincide with known peaks of JH titre in D. melanogaster (Bownes and Rembold, 1987; Sliter et al., 1987), and other species have peaks of JH titre during equivalent periods (Roe and Venkatesh, 1990).

The temporal regulation of synthesis differs between $\mathrm{JH}$ isoforms. The products of the corpus allatum of adult female $D$. melanogaster include between 3 and $23 \%$ JHIII and 1 to $14 \%$ MF. The absolute amounts of MF and JHIII do not change greatly, whereas the dominant in vitro product, $\mathrm{JHB}_{3}$, is temporally regulated, suggesting that MF and JHIII are merely precursors of $\mathrm{JHB}_{3}$ (Altaratz et al., 1991). In 
final instar larvae, however, JHIII and MF production rise and fall approximately in concert with $\mathrm{JHB}_{3}$, although $\mathrm{JHB}_{3}$ is the major product (Richard et al., 1989a), suggesting that all these $\mathrm{JH}$ forms could have a role at this life stage (Fig. 1.2). However, the form of $\mathrm{JH}$ released from the corpus allatum is not necessarily the biologically active form. For example, the corpus allatum of prepupal $M$. sexta is unable to carry out esterification of JH-acid, and the corpus allatum of prepupal $G$. mellonella synthesises JH but also has a high activity of JHE. Thus, both species release JH-acid which is esterified to active JH in other tissues (Sparagana et al., 1985; Wisniewski et al., 1987).

\subsubsection{JH Carrier Proteins}

At least four classes of high affinity, JH-specific carrier proteins are found in the haemolymph of insects (Goodman, 1990; Trowell, 1992, for reviews). Most insects, including Diptera, use lipophorin as their JH carrier. Lipophorin is a high molecular weight lipoprotein (about $300 \mathrm{kDa}$ ) which is abundant in haemolymph. It is the major carrier for lipids but also binds JH at a single specific site. In contrast, the lepidopteran $\mathrm{JH}$ carrier proteins have typical molecular weights of $25-35 \mathrm{kDa}$ and are not associated with significant amounts of lipid.

$\mathrm{JH}$ carriers have at least two roles, the first being to retain $\mathrm{JH}$ in solution. Although $\mathrm{JH}$ is soluble at physiological concentrations it adsorbs to in vitro surfaces and sustained in vitro JH production by corpora allata of the cockroach, Nauphoeta cinerea, requires the presence of the $\mathrm{JH}$ carrier (Lanzrein et al., 1993). Thus, it is assumed that $\mathrm{JH}$ would also adsorb to in vivo surfaces, which might prevent its effective circulation and result in a steep gradient of JH concentration away from its site of production (Trowell, 1992).

A second proposed role is modulation of the activity of JH degrading enzymes (Sanburg et al., 1975) and two types of interaction with these enzymes have been described. The first type of interaction is a simple mass action model, in which the relative abundance and kinetic behaviour of both the carrier and JH degrading enzymes determine the rate of $\mathrm{JH}$ degradation. When the JHE titre is low the carrier is proposed to stabilise $\mathrm{JH}$ in solution but enhance the clearance of JH when JHE activity is high (Hammock et al., 1987). However, in haemolymph of T. $n i$ at times of maximal JHE activity it is the rate of dissociation of $\mathrm{JH}$ from the carrier rather that the abundance of JHE or JH which limits the rate of JH hydrolysis (Abdel-Aal and Hammock, 1988). The second type of interaction is known only in the blattodean, Leucophaea maderae, and requires direct contact for non-competitive inhibition of JHE by lipophorin (Engelmann, 1984; Engelmann et al., 1988).

Differences between $\mathrm{JH}$ isoforms in their binding affinities for the carrier and degrading enzymes suggest a mechanism for the independent regulation of titres of $\mathrm{JH}$ isoforms, although separate roles for $\mathrm{JH}$ isoforms have not been demonstrated. Thus, one isoform could be preferentially degraded while the other is protected by preferential binding to the carrier. This has been shown for the lepidopteran, $G$. mellonella in which only a portion of the total JHII is degraded but hydrolysis eliminates JHIII from circulation at defined developmental stages (Szolajska, 1991). 


\subsubsection{JH Degradation}

The activities of JH hydrolysing enzymes often vary through development in an inverse relationship to $\mathrm{JH}$ titre, suggesting that they play an important role in the regulation of JH titre (Hammock, 1985). The two main degradative pathways for JH are hydrolysis of the ester and the epoxide groups by JHE and JHEH, respectively (de Kort and Granger, 1981; Hammock, 1985; Tobe and Stay, 1985; Roe and Venkatesh, 1990 , for reviews). The products of hydrolysis of JH by JHE and JHEH are JH-acid and $\mathrm{JH}$-diol, respectively, and sequential hydrolysis of $\mathrm{JH}$ by both enzymes can yield JH-acid-diol (Fig. 1.3).

Oxidation and conjugation have also been proposed to inactivate $\mathrm{JH}$. In several Diptera an NADPH dependent oxidase was described as metabolising JH (Slade and Zibitt, 1972; Ajami and Riddiford, 1973; Yu and Terriere, 1978a), but it now appears that this enzyme catalyses the terminal step in the biosynthetic pathway for $\mathrm{JHB}_{3}$. It is found in the $D$. melanogaster ring gland, consistent with a biosynthetic function, but not in the fat body or brain (Richard et al., 1989b) and the peak activities in Musca domestica occur at the times of expected peak $\mathrm{JHB}_{3}$ synthesis in late larval and adult stages (Yu and Terriere, 1978a). In vivo conjugation of the JH hydrolytic products has been demonstrated in many species, with $\mathrm{JH}$-diol and $\mathrm{JH}$-acid being recovered after glucosidase or sulphatase treatments of polar JH metabolites (Roe and Venkatesh, 1990). Conversion to more polar products by hydrolysis and conjugation may be important for excretion of $\mathrm{JH}$ as no unmetabolised $\mathrm{JH}$ was found in the faeces of $M$. sexta (Slade and Zibitt, 1972). Conjugation may also be important to ensure that hydrolysed $\mathrm{JH}$ is not converted back to $\mathrm{JH}$, as $\mathrm{JH}$-acid can be remethylated to form $\mathrm{JH}$, at least in M. sexta imaginal discs (Sparagana et al., 1985).

The hydrolytic pathways for $\mathrm{JH}$ metabolism have been characterised in most detail, with JHE subjected to intensive investigation. These pathways are reviewed in greater detail below.

\subsubsection{Definition of "JH-Specific" Hydrolysis}

An enzyme which can hydrolyse $\mathrm{JH}$ in vitro does not necessarily have specificity for $\mathrm{JH}$ in vivo or a physiological role in regulation of $\mathrm{JH}$ titre so it is important to demonstrate that the enzyme is "JH-specific" (Hammock, 1985). The possibility that an enzyme is not specific can be illustrated by two observations. Firstly, many esterases and epoxide hydrolases have the capacity to hydrolyse substrates in vitro that have no relationship to their biological functions. For example, more than twenty esterases in $D$. melanogaster can hydrolyse the non-physiological ester, naphthylacetate (Healy et al., 1991). Secondly, the commonly used assays for JH hydrolysis usually include JH in considerable excess of physiological concentrations and may not indicate an ability to hydrolyse $\mathrm{JH}$ at low, in vivo concentrations. It should be noted also that resistance to inhibition by the organophosphate diisopropylfluorophosphate (DFP) has sometimes been regarded as diagnostic for JHE. However, while most JHEs are resistant, this alone has no direct bearing on whether JH is the in vivo substrate (Hammock, 1985).

Hammock (1985) has therefore suggested six criteria which singly or in combination could support the application of the term "JH-specific esterase". These criteria, however, can be applied with equal justification to an epoxide hydrolase. Two physiological criteria require demonstration that the enzyme is essential for the clearance of JH from an insect's body, or that increases of enzyme titre correlate with declines of $\mathrm{JH}$ titre. Four biochemical criteria include demonstration that the enzyme has a low $\mathrm{K}_{\mathrm{M}}$ 
for $\mathrm{JH}$, hydrolyses $\mathrm{JH}$ with a high $\mathrm{K}_{\mathrm{cat}} / \mathrm{K}_{\mathrm{M}}$ ratio, rapidly hydrolyses $\mathrm{JH}$ in the presence or absence of $\mathrm{JH}$ carrier protein (indicative of a low $\mathrm{K}_{\mathrm{M}}$ ), or, most rigorously, shows a higher $\mathrm{K}_{\mathrm{cat}} / \mathrm{K}_{\mathrm{M}}$ ratio for JH than for an alternative substrate such as $\alpha$-naphthylacetate. An additional criterion suggested by Abdel-Aal and Hammock (1988) uses the relationship between structure and activity of enzyme inhibitors or alternative substrates to indicate specificity. Enzymes with some or all of these of these properties are discussed below.

\subsection{Physiology of JH Hydrolysis}

\subsubsection{Temporal Profile of JH Hydrolysis}

JH hydrolysis has been studied extensively in the Lepidoptera, with the main focus on hydrolysis in the haemolymph and the events leading up to the pupal moult. During this period JH hydrolysis in the haemolymph occurs exclusively by JHE (Jones, 1986; Roe and Venkatesh, 1990). Key features of this period are the general occurrence of two maxima in the profile of haemolymph JHE activity which coincide with minima in the profiles of JH titre and production (Jones et al., 1982, 1990; Fescemyer et al., 1986; Zimowska et al., 1989). The prewandering JHE maximum appears to coincide with the commitment to pupation (Jones, 1985) and the second JHE maximum occurs immediately prior to pupation and is required for ecdysis (Jones and Hammock, 1985). JHE inhibitors applied at these times cause an increase in $\mathrm{JH}$ titre and a disruption to development, suggesting that the function of JHE is to remove $\mathrm{JH}$ at these times and that JH removal is required for correct development (Jones, 1986). In one lepidopteran, Lymantria dispar, there is an earlier JHE peak, just after ecdysis to the last instar, and a mid pupal peak in addition to the usual two last larval instar peaks, with a decline in JH titre associated with each peak of JHE. In this species JHE activity is higher in females while $\mathrm{JH}$ titre is higher in males for most of this larval/pupal period (Tanaka et al., 1989).

The inverse correlation of $\mathrm{JH}$ hydrolysis with $\mathrm{JH}$ titre and production also occurs in adult females in two insect orders, Blattodea (Tobe et al., 1985) and Diptera (Shapiro et al., 1986; Borovsky et al., 1992) and probably also occurs in Lepidoptera (Venkatesh et al., 1988). In these examples high JH titres and production levels at the onset of vitellogenesis are followed by reduced $\mathrm{JH}$ synthesis and titre and increased JHE activity prior to oviposition. This suggests that JHE also contributes to the reduction of $\mathrm{JH}$ titre in its adult reproductive roles.

However, studies of carefully timed final instar larvae of $M$. sexta (Nijhout, 1975; Roe and Venkatesh, 1990) and T.ni (Jones et al., 1990) and adults of Diploptera punctata (Tobe et al., 1985) and Aedes aegypti (Shapiro et al., 1986) have shown that the JH titre drops to low levels before haemolymph JHE activity rises. Nevertheless JHE inhibitors cause disruptions to metamorphosis and egg production (Jones, 1985; Shapiro et al., 1986; Roe and Venkatesh, 1990), and in prewandering T. ni, the JH titre has been shown to rise slightly when JHE is inhibited (Jones et al., 1990), indicating that JHE is required even when JH titres are low. Quantities of pure JHE, comparable with the final instar JHE maxima, injected into earlier larval stages of $M$. sexta, when $\mathrm{JH}$ is normally high, cause symptoms of only slightly reduced JH titre (Philpott and Hammock, 1990). These results suggest that the role of the highest haemolymph JHE activities is to scavenge the last traces of circulating $\mathrm{JH}$ after 

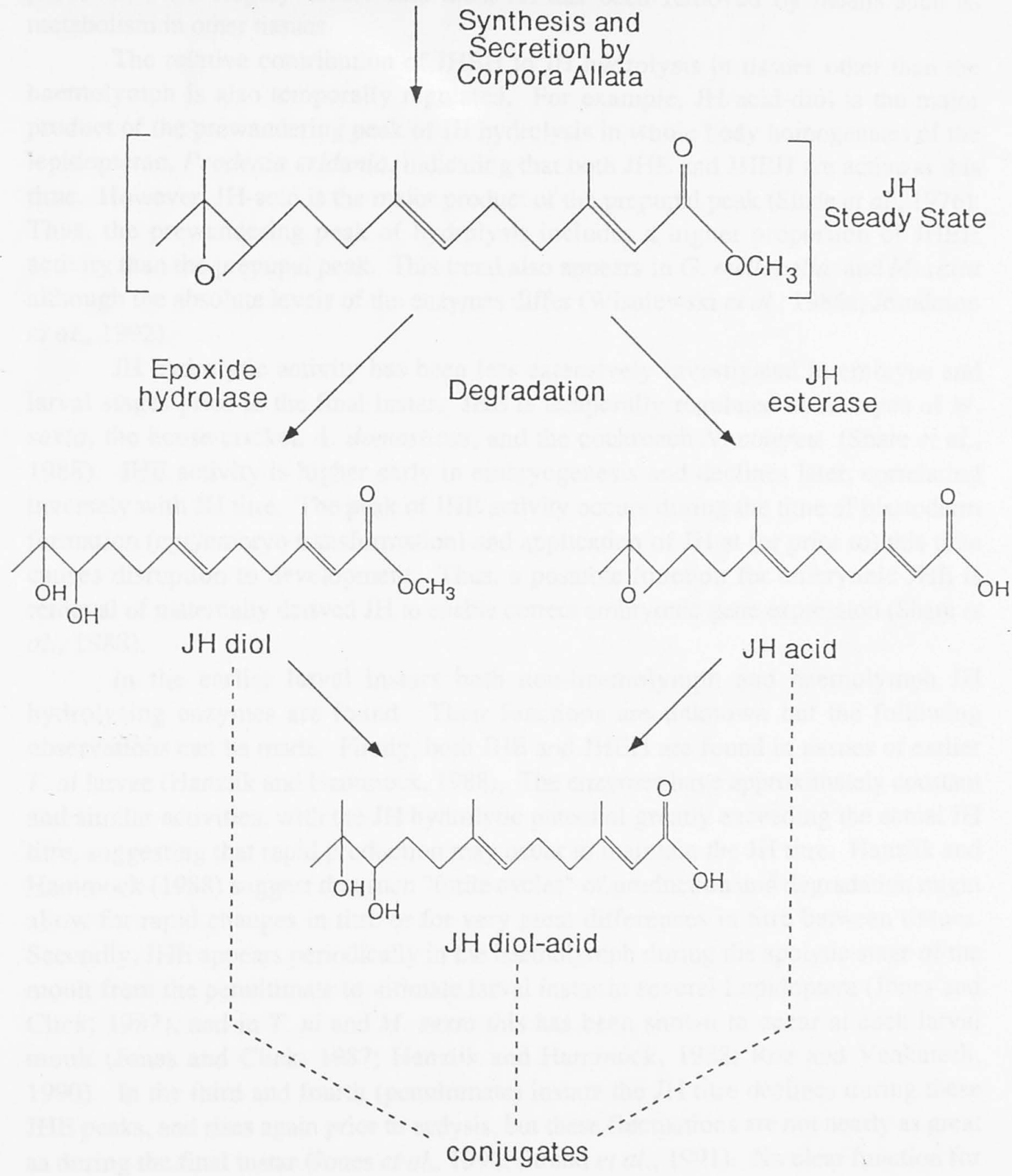

Figure 1.3 Control of Steady State Levels of Juvenile Hormone

Control of $\mathrm{JH}$ titre is generally achieved through both regulated $\mathrm{JH}$ synthesis in the corpus allatum and degradation. The two main routes for degradation are hydrolysis of the epoxide group and the ester bond by $\mathrm{JH}$ epoxide hydrolase and $\mathrm{JH}$ esterase, respectively, yielding the products JH-diol and JH-acid. Further hydrolysis may occur yielding JH-diol-acid. All these products can be conjugated to form more polar products which are excreted. 
production has largely ceased and most $\mathrm{JH}$ has been removed by means such as metabolism in other tissues.

The relative contribution of JHEH to JH hydrolysis in tissues other than the haemolymph is also temporally regulated. For example, $\mathrm{JH}$-acid-diol is the major product of the prewandering peak of JH hydrolysis in whole body homogenates of the lepidopteran, Prodenia eridania, indicating that both JHE and JHEH are active at this time. However, JH-acid is the major product of the prepupal peak (Slade et al., 1976). Thus, the prewandering peak of hydrolysis includes a higher proportion of JHEH activity than the prepupal peak. This trend also appears in G. mellonella and M. sexta although the absolute levels of the enzymes differ (Wisniewski et al., 1986a; Jesudason et al., 1992).

$\mathrm{JH}$ hydrolytic activity has been less extensively investigated in embryos and larval stages prior to the final instar. JHE is temporally regulated in embryos of $M$. sexta, the house cricket, $A$. domesticus, and the cockroach $N$. cinerea (Share et al., 1988). JHE activity is higher early in embryogenesis and declines later, correlating inversely with JH titre. The peak of JHE activity occurs during the time of blastoderm formation (egg/embryo transformation) and application of $\mathrm{JH}$ at (or prior to) this time causes disruption to development. Thus, a possible function for embryonic JHE is removal of maternally derived $\mathrm{JH}$ to enable correct embryonic gene expression (Share et al., 1988).

In the earlier larval instars both non-haemolymph and haemolymph $\mathrm{JH}$ hydrolysing enzymes are found. Their functions are unknown but the following observations can be made. Firstly, both JHE and JHEH are found in tissues of earlier T. $n i$ larvae (Hanzlik and Hammock, 1988). The enzymes have approximately constant and similar activities, with the $\mathrm{JH}$ hydrolytic potential greatly exceeding the actual $\mathrm{JH}$ titre, suggesting that rapid production may occur to maintain the JH titre. Hanzlik and Hammock (1988) suggest that such "futile cycles" of production and degradation might allow for rapid changes in titre or for very great differences in titre between tissues. Secondly, JHE appears periodically in the haemolymph during the apolytic stage of the moult from the penultimate to ultimate larval instar in several Lepidoptera (Jones and Click, 1987), and in T. $n i$ and $M$. sexta this has been shown to occur at each larval moult (Jones and Click, 1987; Hanzlik and Hammock, 1988; Roe and Venkatesh, 1990). In the third and fourth (penultimate) instars the JH titre declines during these JHE peaks, and rises again prior to ecdysis, but these fluctuations are not nearly as great as during the final instar (Jones et al., 1990; Strand et al., 1991). No clear function for JHE during these periods could be demonstrated either by the application of JHE inhibitors or JH analogues (Jones and Click, 1987; Hanzlik and Hammock, 1988).

\subsubsection{Spatial Profile of JH Hydrolysis}

The JHE that is recovered from the haemolymph is believed to be synthesised in the fat body and exported. In $T$. $n i$, JHE activity increases in the fat body at the time of the prepupal peak but following the prepupal haemolymph peak JHE continues to be found in the fat body while its export to the haemolymph is blocked (Wozniak and Jones, 1990).

Metabolism of $\mathrm{JH}$ at sites other than the haemolymph has received less attention. Various studies have identified JHE and/or JHEH activities in dissected tissues such as fat body, epidermis, midgut, brain, suboesophageal ganglion, tracheae, corpora allata 
and cardiaca, silk glands, gonads, and imaginal discs (Roe and Venkatesh, 1990) but only in final instar larvae of the lepidopterans, $M$. sexta, G. mellonella and $T$. ni, have developmental changes of these enzymes within tissues been investigated. In $M$. sexta the specific activities of JHE and JHEH are similiar in the fat body, integument, midgut, and brain, with JHE and JHEH in these tissues and JHE in the haemolymph each accounting for about one third of the total $\mathrm{JH}$ hydrolytic activity (Jesudason et al., 1992). The study indicates that sufficient JH metabolism occurs in tissues to account for $\mathrm{JH}$ titres falling whenever $\mathrm{JH}$ production declines. In most tissues of $M$. sexta, prewandering and prepupal peaks of JHE and JHEH occur in concert. However, there is also evidence of tissue- and stage-specific regulation of these enzymes. For example, in the integument, the prepupal JHE peak is greater than the prewandering peak but the opposite occurs for JHEH, and neither JHE nor JHEH have a prepupal peak in the fat body (Jesudason et al., 1992). A similar pattern was observed in tissues of $G$. mellonella, with JHEH activities generally higher earlier in the final instar and JHE generally higher later in the final instar (Wisniewski et al., 1986a).

The high levels of $\mathrm{JH}$ hydrolysis in tissues suggest that specific tissues could maintain much lower local JH concentrations than occur in general circulation. For example, the increase in brain JHE activity at the time of a small $\mathrm{JH}$ peak in post wandering $G$. mellonella larvae may function to protect neuroblasts which are involved in intense, JH-sensitive formation of neurons (Wisniewski et al., 1986b). Thus, spatial differences of $\mathrm{JH}$ hydrolysis allow the possibility of great variation of $\mathrm{JH}$ titre between tissues and independent regulation of the response of specific tissues to the endocrine environment.

\subsubsection{Regulation of JH Hydrolysis}

Regulation of JHE activity occurs at the levels of synthesis, export from the site of production, specific degradation and modulation of its activity by interactions with other molecules. Regulation of JHEH, however, has not been investigated to the same extent. In $M$. domestica adults, 3 days of exposure to phenobarbital causes JHEH activity to be induced by $78 \%$ (Yu and Terriere, 1978b), and in D. melanogaster, selection by exposure to cut orange over 20 generations caused JHEH, but not JHE, to be increased by $80 \%$ in adults (Ottea et al., 1988). While these results suggest that $\mathrm{JHEH}$ can be regulated, it is not clear how they relate to regulation of $\mathrm{JH}$ titre under natural conditions.

JHE is inducible by its substrate $\mathrm{JH}$ at certain times in larvae, pupae or adults of Lepidoptera, Coleoptera and Blattodea (Roe and Venkatesh, 1990). Induction of JHE by $\mathrm{JH}$ occurs indirectly in prewandering $T$. $n i$ and $M$. sexta but more directly in postwandering larvae. Topically applied $\mathrm{JH}$ induces JHE in the abdomens of prewandering larvae but only if they are not head-ligated. In contrast, induction of JHE by $\mathrm{JH}$ occurs in postwandering larvae with or without head ligation, indicating that a neuroendocrine (head) factor(s) other than $\mathrm{JH}$ is required at the earlier, but not the later, stage (Roe and Venkatesh, 1990).

A JHE-repressive peptide was identified initially from final instar larvae of the lepidopteran, Pseudaletia separata, in which metamorphosis is not initiated due to parasitism by the wasp, Apantales kariyai (Hayakawa, 1990, 1991). The peptide is also present in the haemolymph of the unparasitised host during the penultimate instar and may be a previously undescribed hormone (Hayakawa, 1992). In all three lepidopteran 
species tested, the peptide reduces weight gain in the final instar and delays pupation but it is not clear whether JHE repression is the cause or a consequence of the block to growth and development (Hayakawa and Yasuhara, 1993).

The T. ni JHE must be glycosylated before it is exported from its site of synthesis in the fat body. However, after the prepupal peak of haemolymph JHE, high levels of JHE continue to be present in the fat body and a factor other than synthesis or glycosylation in pupae prevents its export to the haemolymph (Wozniak and Jones, 1990).

Lepidopteran larvae also have a specific mechanism for the removal of JHE from the haemolymph. Exogenous Heliothis virescens JHE has a half life of only 1.2-3.6 hours in the haemolymphs of $H$. virescens or $M$. sexta, being rapidly and specifically taken up into lysosomes of haemocytes and pericardial cells at the developmental times when endogenous JHE activity is known to fall (Booth et al., 1992; Ichinose et al., 1992a,b). JHE taken into the pericardial cells could not be recovered from homogenised pericardial complexes without the use of a protease inhibitor cocktail, indicating that JHE is rapidly degraded (Ichinose et al., 1992b).

Lipophorin, described in section 1.5.2, may interact directly with JHE as a noncompetitive inhibitor (Engelmann et al., 1988) and other endogenous inhibitors or activators of JHE may exist. $M$. sexta JHE is greatly activated in the presence of molar concentrations of a wide range of water-miscible organic solvents (Croston et al., 1987). The solvents might act at a relatively hydrophobic modifier site that has an $i n$ vivo activation role. However, other lepidopteran JHEs were only activated modestly or inhibited under these conditions.

\subsubsection{JH Hydrolysis in Diptera}

JH degradation in many insects plays a specific, albeit not fully understood, role in the regulation of $\mathrm{JH}$. In the Diptera, however, the role of $\mathrm{JH}$ degradation is far from clear. The use of the non-physiological JHI until the early 1980s and the small number of studies limit generalisation about dipteran $\mathrm{JH}$ metabolism. With this caveat in mind however, some comments on the temporal and spatial patterns of expression of dipteran JH hydrolysing enzymes can be made.

The available evidence from larvae suggests that prewandering and prepupal peaks of hydrolysis may occur. Microsome preparations of $M$. domestica larvae exhibit a prewandering peak of JHEH activity (Yu and Terriere, 1978b) and JH hydrolysis occurs in final instar larvae and pupae of Drosophila virilis with a minimum of activity just prior to pupariation (Rauschenbach et al., 1991). A prepupal peak of JHE activity occurs in D. hydei (Klages and Emmerich, 1979) and rates of JH metabolism in whole pupae of $M$. domestica, Chaoborus americanus, and $D$. melanogaster are comparable to other orders (Ajami and Riddiford, 1973). JH hydrolysis is temporally regulated in two adult female dipterans. In $A$. aegypti, JHE activity varies inversely with $\mathrm{JH}$ titre in response to a blood meal (Shapiro et al., 1986; Borovsky et al., 1992) and this is similar to temporal variations in JHE and $\mathrm{JH}$ titres observed in other insect orders during cycles of egg production (Renucci et al., 1984). In D. melanogaster, microsomal JHEH activity was found in 2 day old whole adult virgin females but not in 6 day old virgin females, whereas microsomal JHE activity is similar at both ages (Ottea et al., 1988). While temporal regulation of $\mathrm{JH}$ production in $D$. melanogaster has been 
demonstrated (Altaratz et al., 1991), it is not known whether hydrolysis of JH also plays a part in the regulation of JH titre during vitellogenesis in this species.

Non-haemolymph metabolism of JH may be of particular importance in the Diptera. The haemolymph of Sarcophaga bullata (Weirich and Wren, 1976), D. hydei (Klages and Emmerich, 1979) and D. melanogaster (Wilson and Gilbert, 1978; Shemshedini and Wilson, 1988) larvae contains little JH hydrolytic activity, unlike most other insects investigated. Nevertheless, JHE and JHEH activities are present in whole D. melanogaster (Wilson and Gilbert, 1978), microsome preparations of $M$. domestica larvae (Yu and Terriere, 1978a), and the body wall and fat body of $D$. hydei larvae (Klages and Emmerich, 1979). The finding of multiple forms of JHEH in D. melanogaster final instar larvae with differing activities towards $\mathrm{JHII}$ and $\mathrm{JHB}_{3}$ and differing subcellular localisations might reflect local regulation of $\mathrm{JH}$ within tissues, perhaps with differential regulation of JHIII and $\mathrm{JHB}_{3}$ (Casas et al., 1991; Harshman et al., 1991). Most JHE and JHEH activities occur in the microsomal fraction of whole, female $M$. domestica (Yu and Terriere, 1978a), suggesting an intracellular location. Furthermore, in adult $M$. domestica, haemolymph JHE activity was the lowest found in a comparative study across six insect orders, yet the half life of injected JHI was lowest in this species (de Kort et al., 1979). These observations suggest that considerable JH degrading activity occurs in non-haemolymph sites. Although $\mathrm{JH}$ degradation appears to be generally absent or low in the haemolymph of larvae and adults, JHE occurs in the haemolymph of $D$. hydei prepupae and persists though declining through the pupal stage (Klages and Emmerich, 1979). However, other pupal tissues were not investigated.

In $D$. virilis an electrophoretically detected esterase (denoted "p-esterase" for pupal esterase) has been extensively studied since the early 1970s and is proposed to be JHE. Several lines of evidence led to this proposal: it is induced by $\mathrm{JH}$ and $\mathrm{JH}$ analogues; it follows the expected developmental profile for JHE, with predominantly pupal expression; JHI appeared to competitively inhibit its hydrolysis of naphthylacetate in an "in gel" assay; JH hydrolytic activity was only observed in homogenates with pesterase activity; and it was the only naphthylacetate esterase not to be inhibited by DFP (Rauschenbach et al., 1987, 1991 and earlier references therein). Furthermore, after anion exchange chromatography of pupal homogenates, JH hydrolytic activity was recovered from two fractions, one of which also contained the p-esterase (Dr. I. Y. Rauschenbach, pers. comm.).

\subsection{Biochemistry of Juvenile Hormone Esterases}

\subsubsection{In Vitro Inhibitors of JHE}

The active sites of lepidopteran JHEs have been extensively probed using trifluoromethylketones (Fig. 1.4), a class of esterase inhibitors (Roe et al., 1990 and references therein). The trifluoromethylketone group mimics the transition state of ester hydrolysis and therefore binds with high affinity in the active site of esterases (Linderman et al., 1993). Several series of compounds which incorporate the trifluomethylketone group were tested as inhibitors of lepidopteran JHEs to uncover determinants of specific binding to JHE. Aliphatic trifluoromethylketones with chain lengths similar to the backbone of $\mathrm{JH}$ were found to be especially good inhibitors and 
<smiles>COC(=O)/C=C(\C)CC/C=C(\C)CCC1OC1(C)C</smiles><smiles>CCCCCCCC([18O])SC(C(=O)O)C(=O)C(F)(F)F</smiles><smiles>CC(C)OP(=O)(F)OC(C)C</smiles>

DFP

Figure 1.4 Structures of Esterase Inhibitors Compared with JHIII

The structures of the trifluoromethylketone inhibitor, OTFP and the organophosphorus inhibitor, DFP are compared with JHIII. The positions of $\alpha$ and $\alpha^{\prime}$ methyl substitutions which enhance the activity of OTFP are indicated (Linderman et al., 1987). 
quite selective for JHE over other esterases; the most effective inhibitor (3-(octylthio)1,1,1-trifluoropropan-2-one, OTFP) had a chainlength of twelve. It also incorporates a sulphur atom into the chain which is proposed to mimic the 2,3 double bond (Linderman et al., 1987). Neither the addition of side chains at C7 and C11, nor the introduction of further double bonds into the chain, enhances inhibition, although these changes were expected to better mimic the structure of JH (Linderman et al., 1989). Addition of an $\alpha$ - or $\alpha$-methyl group, however, does enhance inhibition by OTFP but an $\alpha$-ethyl addition reduces inhibition (Linderman et al., 1987). This was interpreted as indicating that a small hydrophobic pocket exists adjacent to the carbonyl binding site of JHE which favours binding of the $\mathrm{C} 3$ methyl substitution found on JH but not larger groups. This interpretation is consistent with the observation that most non-dipteran JHEs are very resistant to inhibition by the organophosphate, DFP $\left(\mathrm{I}_{50}>10^{-3} \mathrm{M}\right)$ (Hammock, 1985). Organophosphates which have less bulk adjacent to the phosphate than DFP are effective inhibitors of JHEs, suggesting that poor inhibition by DFP is due to steric hinderance (Roe et al., 1990).

Recently, OTFP and $\alpha$-methylOTFP have been modified by replacement of the carbonyl moiety with an alcohol moiety (Linderman et al., 1993). The modified compounds are each less potent inhibitors of JHE than their parent compounds. However, they are more selective for $T$. ni JHE over $\alpha$-naphthylacetate hydrolysing esterases from $T . n i$ haemolymph and electric eel acetylcholine esterase (AChE) and so may prove more useful probes of JHE function in vivo. The selectivity of these compounds for JHE suggests that hydrogen bonding plays a different role in the binding of the $\mathrm{JH}$ transition state than in the transition state binding of other esterases.

Dipteran JHEs have been less extensively investigated but their profiles of sensitivity to inhibitors differ from other JHEs. JHEs from non-dipteran insect orders are generally resistant to DFP $\left(\mathrm{I}_{50}>10^{-3} \mathrm{M}\right.$, Hammock, 1985), although two exceptions are the orthopterans, Gryllus rubens, $\left(\mathrm{I}_{50}=10^{-5} \mathrm{M}\right.$, Zera et al., 1992), and Locusta migratoria (Peter et al., 1979). In contrast, the JHEs of the dipterans, S. bullata and Phormia regina but not $M$. domestica, are sensitive to DFP ( $\mathrm{I}_{50}$ about $10^{-4}$ $\mathrm{M}, \mathrm{Yu}$ and Terriere, 1978a) and D. virilis may have both DFP-sensitive and DFPresistant forms of JHE (Dr. I. Y. Rauschenbach, pers. comm.). M. domestica JHE exhibits an atypical pattern of sensitivity to a range of organophosphates, carbamates and other esterase inhibitors (Mumby et al., 1979; Sparks and Hammock, 1980) and Culex pipiens pipiens JHE is resistant to several effective inhibitors of other JHEs (Hooper, 1976). Taken together these atypical inhibitor sensitivities suggest structural differences between the active sites of dipteran and non-dipteran JHEs.

\subsubsection{Purification of JHEs}

JHE has been purified to homogeneity from larval haemolymph of seven species of Lepidoptera but not from any other order. Classical chromatographic techniques have been used to purify JHEs from M. sexta (Couldron et al., 1981), T. ni (Yuhas et al., 1983; Rudnicka and Jones, 1987; Wozniak et al., 1987), G. mellonella (Rudnicka and Kochman, 1984), and L. dispar (Valaitis, 1991; 1992). Recoveries and enrichment factors were $1-40 \%$ and $383-4000$ fold. Each of these purifications required four or five steps, with most including either polyethyleneglycol (PEG) or ammonium sulphate precipitation followed by gel filtration and ion exchange chromatography, and several 
included hydroxylapatite chromatography. Particularly successful methods used in the more recent purifications involved concanavalin A binding, which relies on the presence of polysaccharides on the surface of JHE (Valaitis, 1992), and preparative isoelectric focussing (IEF, Rudnicka and Jones, 1987; Wozniak et al., 1987).

Affinity chromatography has been used to purify JHEs from the haemolymph of M. sexta, Bombyx mori, H. virescens and Heliothis zea (Abdel-Aal and Hammock, 1985; 1986; Abdel-Aal et al., 1988), and from haemolymph and whole organism homogenate of prepupal larvae of T. ni (Hanzlik and Hammock, 1987). This method uses trifluoromethylketone compounds (Section 1.7.1) as both the ligand and the eluting agent (OTFP). Recoveries from haemolymph were 50-98\% with 585-1240 fold enrichment, whereas the recovery and enrichment factor from $T . n i$ larval homogenate were $10 \%$ and 1340 fold, respectively.

All the purified JHEs consist of a single polypeptide in the range of $62-68 \mathrm{kDa}$ which is typical of other esterases (Cygler et al., 1993), and the JHEs of M. sexta, T. $n i$ and L. dispar are monomeric.

\subsubsection{Kinetic Characterisation of JHEs}

Kinetic analyses of non-purified JHEs have been performed using diluted haemolymph. The JHEs exhibited $\mathrm{K}_{\mathrm{MS}}$ of around $100 \mathrm{nM}$ in the Lepidoptera (Wing et al., 1984), $1570 \mathrm{nM}$ and $670 \mathrm{nM}$ for two JHEs in the blattodean L. maderae (Gunawan and Engelmann, 1984), $500 \mathrm{nM}$ in the coleopteran Leptinotarsa decemlineata (Kramer and de Kort, 1976), and 47-81 nM in the orthopteran G. rubens (Zera et al., 1992). The use of unpurified enzyme generally prevents complete kinetic analysis because interference from carriers or other modulators of JHE activity may occur and quantification of the amount of enzyme in such mixtures is often difficult. An exception is the study of Abdel-Aal and Hammock (1988) in which OTFP was used as a stoichiometric inhibitor of JHE in whole $T$. $n i$ haemolymph to determine the enzyme molar equivalency. Thus, effects of JH binding by both the carrier and JHE were included in the kinetic analysis, and a $\mathrm{K}_{\mathrm{cat}}$ of $0.53 \mathrm{sec}^{-1}$ and a $\mathrm{K}_{\mathrm{M}}$ of $70.6 \mathrm{nM}$ for the hydrolysis of JHII were determined.

The only dipteran JHE subjected to kinetic analysis is from cytosol of $D$. hydei late larval integument (Bisser and Emmerich, 1981). The apparent $\mathrm{K}_{\mathrm{M}}$ for JHI is $640 \mathrm{nM}$ and competition data suggest a similar affinity for JHIII and, surprisingly, a greater affinity for the ethyl ester of JHIII. However, it is not known whether any competing JH-binding proteins were present in the cytosol preparation. Affinity for MF appears to be about ten-fold lower than for JHIII and about 100 fold lower for the JHanalogue, methoprene.

Kinetic analysis reveals that most purified JHEs from Lepidoptera have very low $\mathrm{K}_{\mathrm{MS}}$ for $\mathrm{JH}(21-360 \mathrm{nM})$ and quite low turnover numbers $\left(\mathrm{K}_{\mathrm{cat}}, 0.49-4.33 \mathrm{sec}^{-1}\right)$ (Rudnicka and Kochman, 1984; Abdel-Aal and Hammock, 1985; Hanzlik and Hammock, 1987; Rudnicka and Jones, 1987; Wozniak et al., 1987; Abdel-Aal et al., 1988; Valaitis, 1991, 1992; Ward et al., 1992). One preparation of T. ni JHE had a higher $\mathrm{K}_{\mathrm{M}}(1130 \mathrm{nM}, \mathrm{JHI} ; 402 \mathrm{nM}, \mathrm{JHIII})$ and lower $\mathrm{K}_{\mathrm{cat}}\left(0.046 \mathrm{sec}^{-1}\right.$, Yuhas et al., 1983) but was nine-fold less enriched than other T. ni JHE preparations. Maximum in vivo $\mathrm{JH}$ concentrations in insects are generally very low. $(1-100 \mathrm{ng} / \mathrm{ml}$ or $3.6-360 \mathrm{nM}$, Schooley et al., 1984) and so the $\mathrm{K}_{\mathrm{MS}}$ of the lepidopteran JHEs are similar to the maximum $\mathrm{JH}$ titres. The specificity constants $\left(\mathrm{K}_{\mathrm{cat}} / \mathrm{K}_{\mathrm{M}}\right)$ of these enzymes 
(1.4-58 $\times 10^{6} \mathrm{M}^{-1} \mathrm{sec}^{-1}$ ) determine the rate of hydrolysis when the substrate concentration is much lower than the $\mathrm{K}_{\mathrm{M}}$ (Abdel-Aal and Hammock, 1988), and approach the diffusion limited maximum value $\left(10^{8}-10^{9} \mathrm{M}^{-1} \mathrm{sec}^{-1}\right.$, Stryer, 1981). These data indicate that lepidopteran JHEs are close to optimally adapted for efficient hydrolysis of $\mathrm{JH}$ from low concentrations, which is consistent with their proposed role as scavengers of trace amounts of $\mathrm{JH}$ at times in development when very low $\mathrm{JH}$ titres are required (Roe and Venkatesh, 1990).

\subsubsection{Intraspecific and Interspecific Differences Among JHEs}

The haemolymphs of several lepidopterans and two orthopterans contain multiple, closely similar forms of JHE which can be separated by high resolution IEF. However, the nature of the isozyme variation is not always clear and the function of intraspecific isozyme diversity, if any, is unknown (Wing et al., 1984; Zera et al., 1992).

It has been hypothesised that variants of $T . n i$ JHE reflect differences in glycosylation or may be a consequence of aggregation as kinetic differences are small (Jones et al., 1986; Hanzlik and Hammock, 1987). However, glycosylation differences probably do not account for the electrophoretic variation as the glycosylation of each variant is heterogeneous. These variants do not represent precursors and mature forms of JHE because they are coordinately induced by JH in the fat body, suggesting that they either derive from a common, hypothetical and undetectable precursor or from different transcripts (Wozniak and Jones, 1990). In H. virescens two variants differ by an N-terminal extension of two amino acids on one variant (Hanzlik et al., 1989) and slight differences in amino acid composition also occur between JHE variants in both T. ni (Wozniak et al., 1987) and $L$. dispar (Valaitis, 1991). The T. ni JHEs are immunologically indistinguishable and the two variants in $L$. dispar have conservative amino acid substitutions in homologous peptides, suggesting that they are allelic variants. Purified M. sexta JHE which is homogeneous by electrophoretic (Abdel-Aal and Hammock, 1985) and N-terminal sequence (Dr. T. N. Hanzlik, pers. comm.) criteria has two apparent $\mathrm{K}_{\mathrm{M}}$ s and a point of inflection on inhibitor curves which indicates two equally abundant active sites. The authors suggest that $M$. sexta JHE has two active sites but it is also possible that the preparation contained equal amounts of two similar JHEs. No known esterase has more than one active site per subunit (Cygler et al., 1993), although this feature has been postulated for AChE (Small, 1990).

Kinetic analysis reveals two forms of the JHE active site in diluted $H$. virescens haemolymph ( $\mathrm{K}_{\mathrm{MS}}=16 \mathrm{nM}$ and $250 \mathrm{nM}$, Wozniak and Jones, 1987). There are also two electrophoretic variants but only the lower $\mathrm{K}_{\mathrm{M}}$ can be detected after elution of either electrophoretic variant from IEF gels. Thus, it is unclear whether these results indicate separation of JHE from a modifier molecule or loss of activity by a higher $\mathrm{K}_{\mathrm{M}}$ variant during electrophoresis. In $G$. mellonella two JHE variants copurify but can be separated by IEF. At least one difference between these variants has an influence on the active site because they differ in their ability to hydrolyse naphthylacetate (Rudnicka and Kochman, 1984).

In spite of attempts to identify functional differences between intraspecific variants, little information is available. The same haemolymph variants appear throughout development in M. sexta and T. ni (Hanzlik and Hammock, 1988; Jesudason et al., 1990). In the cricket $G$. rubens the presence of a second haemolymph 
JHE variant correlated with wing morph, suggesting a possible functional difference between the two variants (Zera et al., 1992). Non-haemolymph JHE variants in some cases exhibit the same distribution of isoelectric points as the haemolymph variants (Wozniak and Jones, 1990) but in other cases they are different (Hanzlik and Hammock, 1987; Sparks et al., 1989).

In the Diptera little data are available on intraspecific variation. However, soluble and membrane associated JHEs in $M$. domestica exhibit different stability and inhibitor sensitivities, which may reflect a functional difference between two variants (Sparks and Hammock, 1980).

The lepidopteran JHEs also exhibit substantial interspecific differences. JHEs from two Heliothis species are antigenically distinct, reflecting considerable differences in substrate selectivity, inhibitor sensitivity and response to organic co-solvents (Section 1.6.3, Croston et al., 1987; Abdel-Aal et al., 1988). However, within the superfamily Noctuoidea, some, but not all, JHEs exhibit immunological cross-reactivity with $T$. $n i$ JHE (Wozniak and Jones, 1987; Valaitis, 1991). Immunological relationships outside the Noctuoidea were investigated using antisera raised against JHEs of Heliothis virescens (Noctuioidae), M. sexta (Sphingoidae) and T. ni (Noctuioidae) to probe western blots of JHEs from these three species, Heliothis zea (Noctuioidae) and Bombyx mori (Bombycoidea) (Hanzlik, 1988). H. virescens antibodies detected all five JHEs, $M$. sexta antibodies recognised both $B$. mori and some of the Noctuoidea JHEs, but $T$. $n i$ antibodies only recognised the JHEs from the same superfamily. These complex immunological relationships suggest that while some epitopes are conserved between all the lepidopteran JHEs, considerable interspecific differences occur. The apparently conflicting data might be resolved in part by considering the epitopes recognised by particular antisera. For example, antibodies generated against $L$. dispar JHE from haemolymph recognise JHEs from other species, yet fail to recognise L. dispar JHE from the fat body which lacks carbohydrate, suggesting that this antiserum mainly recognises the carbohydrate moiety (Valaitis, 1992).

Amino acid sequence data also suggest considerable difference among the lepidopteran JHEs. Sequences of peptide fragments of $L$. dispar JHE totalling more than 300 residues (over $50 \%$ of the whole protein) were difficult to align with the $H$. virescens complete JHE sequence (Valaitis, 1991) and there is little similarity between the three published $\mathrm{N}$-terminal sequences of lepidopteran JHEs $(H$. virescens, complete cDNA, Hanzlik et al., 1989; L. dispar, 69 residues, Valiatis, 1992; M. sexta, 15 residues, Venkatesh et al., 1990).

Several lines of evidence suggest that dipteran JHEs will differ substantially from the lepidopteran JHEs. Firstly, the lepidopteran JHEs show considerable interspecific differences and are expected to show even greater divergence from JHEs of other insect orders. Secondly, the inhibitor sensitivities of lepidopteran and dipteran JHEs differ (Section 1.7.1). Thirdly, the cDNA of $H$. virescens JHE (Hanzlik et al., 1989) does not hybridise with D. melanogaster genomic DNA (Ms. S. J. Dent, pers. comm.), and fourthly, $H$. virescens JHE antibodies do not cross-react with D. melanogaster proteins (Ms. M. M. Dumancic, pers. comm.). 


\subsection{Characterisation of the JHE Gene}

The JHE gene has been isolated and characterised from one lepidopteran, $H$. virescens. Its inferred amino acid sequence shows about $24 \%$ identity with several esterases including AChE (Hanzlik et al., 1989). High levels of conservation occur around particular features that identify JHE as a member of a multigene family which includes other carboxylesterases, cholinesterases and lipases. X-ray structures have been determined for two members of the family and this has enabled functional roles of conserved sequences to be inferred (Cygler et al., 1993). Generally conserved features include the residues and position of the catalytic triad in the primary sequence, hydrophobic core-forming residues, residues involved in salt bridges, cysteine residues forming disulphide bridges, and residues at the edges of secondary structural elements. In $H$. virescens JHE, the catalytic triad suggested by sequence comparison (Ser201, Glu332, and His446) is supported by in vitro mutagenesis experiments (Ward et al., 1992). H. virescens JHE lacks one of the generally conserved cysteine pairs but its location in a surface loop near the active site suggests that its role might be substrate binding rather than maintainance of structure.

The catalytic triad mechanism used for hydrolysis by the esterases/lipases is found in a number of enzyme families. It is proposed that the esterase/lipase family is part of a larger group of hydrolases which is characterised by a common structural feature, the " $\alpha / \beta$ hydrolase fold" (Ollis et al., 1992). This structure determines a consistent spacial arrangement of the catalytic triad, and is proposed to have been conserved among enzymes no longer exhibiting detectable sequence similarity and having diverse substrates. The serine proteases use the same catalytic mechanism as the esterase/lipase family although the order of the triad residues in the primary sequence and other structural features are different. Therefore, the use of a catalytic triad by the serine proteases as well as two other classes of hydrolases is believed to be an example of convergent evolution (Ollis et al., 1992).

Among the cloned esterases only AChE and JHE have well defined in vivo substrates and so these enzymes are of particular importance for the understanding of structure/function relationships in this multigene family.

\subsection{Aims and Outline of This Study}

The aim of this project was to characterise $\mathrm{JH}$ degradation in D. melanogaster. The physiological and biochemical properties of the $\mathrm{JH}$ degrading enzymes in Diptera are compared with those in other insect orders, with particular emphasis on JHE. Having defined the dipteran $\mathrm{JH}$ degrading enzymes a further aim was to assess the relative contributions of degradation to regulation of $\mathrm{JH}$ titre between Diptera and Lepidoptera.

No systematic study of $\mathrm{JH}$ hydrolysis had been performed previously in D. melanogaster although JHE and JHEH were known to occur in larvae, pupae and adults (Ajami and Riddiford, 1973; Wilson and Gilbert, 1978; Ottea et al., 1987). In other Diptera, JHE is temporally regulated in $D$. hydei prepupae (Klages and Emmerich, 1979) and during vitellogenesis in adult female A. aegypti (Shapiro et al., 1986) and has a low $\mathrm{K}_{\mathrm{M}}$ for JHI in larval epidermis of D. hydei (Bisser and Emmerich, 1981). Together these data suggest that $D$. melanogaster JHE may have a role in the regulation of $\mathrm{JH}$ titre as has been established in other orders (Section 1.5). However, 
little or no JHE is found in the haemolymph of Diptera, in contrast to most other insects (Section 1.6), and dipteran JHEs have an atypical pattern of inhibitor sensitivity (Section 1.7.1), suggesting that both the expression pattern and biochemical properties of the dipteran JHEs may be atypical.

Chapter two describes the identification and physiological characterisation of $\mathrm{JH}$ hydrolytic enzymes in $D$. melanogaster. Two approaches were taken to the identification of the JH hydrolysing enzymes, the first being to determine whether any of the electrophoretically detected esterases of $D$. melanogaster is JHE. In D. virilis the electrophoretically detected p-esterase was proposed to be JHE (Rauschenbach et al., 1987) and preliminary work suggested that an homologous esterase may be present in D. melanogaster (Healy et al., 1991). An esterase, EST20, visualised after native polyacrylamide gel electrophoresis (native PAGE) using histochemical techniques was found to be very similar to the p-esterase of $D$. virilis. However, it was also found to not exhibit JH hydrolysing activity. The second approach was to use a radiometric assay with JHIII as the substrate (Hammock and Roe, 1985). Two JH hydrolysing enzymes, JHE and JHEH were identified using this approach. Investigation of the temporal regulation of these enzymes indicated that the activities of both enzymes increase at times of known declines of JH titre, thereby satisfying one of the proposed physiological criteria for JH-specificity (Section 1.5.3.1). Further work then concentrated on characterisation of JHE from the time of its maximum expression.

Chapter three describes the purification and kinetic characterisation of JHE. An affinity purification procedure previously used for lepidopteran JHEs was applied to the purification of $D$. melanogaster JHE but did not yield active enzyme. A conventional chromatographic protocol for the purification of JHE was then developed and the protein purified by this procedure was used for $\mathrm{N}$-terminal amino acid sequencing and kinetic studies. Kinetic experiments were used to assess the specificity of JHE for JH, JHE selectivity between $\mathrm{JH}$ isoforms, the features of the $\mathrm{JH}$ molecule that determine JHE specificity, and the nature of any interaction between JHE and the JH carrier, lipophorin. JHE was found to have a low $\mathrm{K}_{\mathrm{M}}$ for JHIII and a high $\mathrm{K}_{\text {cat }} / \mathrm{K}_{\mathrm{M}}$, confirming that it is capable of efficient hydrolysis of JH at low, physiological concentrations. It has high binding affinity for both JHIII and the natural stereoisomer of $\mathrm{JHB}_{3}$, indicating that either or both of these $\mathrm{JH}$ isoforms could be the in vivo substrate for JHE. Experiments with a wide variety of JH analogues indicated that most features of the JH molecule contribute to recognition by the active site of JHE but the 10,11 epoxide in the natural $\mathrm{R}$ conformation is of particular importance. Lipophorin was found to reduce the rate of JH hydrolysis by JHE but the effect was due only to competition for binding of $\mathrm{JH}$. Together these results indicate that JHE is specific for JH by each of the proposed biochemical criteria (Section 1.5.3.1).

In Chapter four the results from chapters two and three are combined with data from the literature to develop a model of JH hydrolysis in Diptera. This model is compared and contrasted with JH hydrolysis in Lepidoptera and other insect orders. JH specificity and an inverse relationship between the developmental profiles of the JH hydrolytic enzymes and the profiles of JH production and JH titre indicate an important role for $\mathrm{JH}$ hydrolysing enzymes in the regulation of $\mathrm{JH}$ titre. This proposal is further supported by similarities between the patterns of JH hydrolysis in the Diptera and Lepidoptera although there are some important differences. The possibility of differential regulation of the $\mathrm{JH}$ isoforms, JHIII and $\mathrm{JHB}_{3}$, through an interplay of regulated hydrolysis, production and carrier binding, is discussed. 


\section{Chapter 2}

\section{Identification and Expression Pattern of JH Degrading Enzymes}

\subsection{Introduction}

The work described in this chapter had two aims. The first was to identify the $\mathrm{JH}$ degrading enzymes of $D$. melanogaster and the second was to determine their importance for regulation of $\mathrm{JH}$ titre. The available data indicate that while JHE and JHEH both occur in Diptera, including D. melanogaster, their patterns of expression and physical properties differ from those seen in other insects (Sections 1.6.4 and 1.7.1). This chapter describes two approaches used to identify and characterise $\mathrm{JH}$ degrading enzymes in $D$. melanogaster.

The first approach was to investigate esterases of $D$. melanogaster fractionated by electrophoresis and visualised using the artificial esterase substrate, naphthylacetate. Electrophoretically detected esterases from the lepidopteran, Hyalophora gloveri, and the dipteran, Drosophila buzzatii, have been postulated to be JHE on the grounds of induction by $\mathrm{JH}$, elution of $\mathrm{JH}$ hydrolytic activity from gel slices after electrophoresis, or expression at times of presumed reduction of JH titre (Whitmore et al., 1972; East, 1982). Furthermore, the most extensive study of a dipteran enzyme believed to be involved with $\mathrm{JH}$ degradation is the electrophoretically detected "p-esterase" of D. virilis (Rauschenbach et al., 1987, 1991, and references cited therein). Fractionation of esterases by native PAGE reveals similarities in the number, relative mobility and substrate specificity of esterases among Drosophila species and these properties enabled initial identification of homologues of D. melanogaster EST6 in other Drosophila species, including D. virilis (Oakeshott et al., 1990). The substrate specificity and expression pattern of the p-esterase, together with its high mobility on native PAGE, are consistent with the properties of the "anodal" esterases described in five Drosophila species, including D. melanogaster (Berger and Canter, 1973; Rauschenbach et al., 1977; Healy et al., 1991). Thus, my initial hypothesis was that the JHE of D. melanogaster would be found among the anodal esterases of this species.

In the second approach, JH hydrolysis was measured directly using a radiometric assay which detects both $\mathrm{JHE}$ and JHEH. This approach has been used extensively to identify $\mathrm{JH}$ hydrolysing enzymes in many insect orders including the Diptera (Hammock, 1985; Hammock and Roe, 1985). Therefore, this technique was used to identify the $\mathrm{JH}$ hydrolysing enzymes in whole body homogenates of D. melanogaster both prior to, and after, electrophoretic and subcellular separations.

The second aim of this study was to determine the importance of $\mathrm{JH}$ degradation for regulation of JH titre. Hammock (1985) suggested two physiological criteria for determining the $\mathrm{JH}$ specificity of enzymes that hydrolyse $\mathrm{JH}$ in vitro (Section 1.5.3.1). One of these requires that increases of enzyme activity correlate with declines of JH titre and this criterion is fulfilled by JHEs from haemolymph of final instar lepidopterans (Section 1.6.1). Among the Diptera, these criteria have only been satisfied for JHE in adult female A. aegypti, although comparison of data between dipteran species suggests that increased $\mathrm{JH}$ hydrolysis may also occur during the final instar of dipterans at times 
of reduced JH titre (Section 1.6.4). In this study the developmental profiles of JHE and JHEH from $D$. melanogaster were determined using a radiometric assay and compared with data from the literature for the developmental profiles of $\mathrm{JH}$ titre and production.

\subsection{Materials and Methods}

\subsubsection{Drosophila Strains, Culture and Sample Preparation}

\subsubsection{Strains and Culture}

The D. melanogaster strain 12I11.2 (Cooke et al., 1987) which expresses the anodal esterases more reliably than other strains (Healy et al., 1991 and unpublished data) was used, except where Est6 $6^{\text {null }} r y$ (Oakeshott et al., 1987) is indicated. Est $6^{\text {null }}$ ry carries a null allele for Est6 which produces no EST6 protein. The strain of D. virilis used was Texmelucan.

Flies were routinely raised in a 12 : 12 hour light : dark cycle at $25^{\circ} \mathrm{C}, 65 \%$ humidity, on agar-cornmeal-molasses medium. Cultures were kept at low density by allowing only a small number of eggs to be laid in bottles. Staged larvae were cultured from eggs laid within two hours. Pupae were staged by the flotation method of Mitchell and Mitchell (1964). Adults were staged by collecting those which emerged within a twelve hour period. Males and females were then aged separately.

The effect of culture density on esterase expression was determined after counting 10-200 eggs into vials containing $13 \mathrm{ml}$ of medium. Prepupae/pupae were collected from the vials every twelve hours until all larvae had pupariated.

To determine the fate of esterases at eclosion, pupae that had been staged to within two hours were incubated until about half the adults had emerged. The remaining pharate adults, the discarded pupal cases and the newly emerged adults were collected and homogenised as described below.

\subsubsection{Sample Preparation}

Except where indicated, all samples were frozen in liquid nitrogen and stored at $-70^{\circ} \mathrm{C}$ prior to homogenisation in $100 \mathrm{mM}$ sodium phosphate buffer, $\mathrm{pH} 6.8$ containing $0.5 \mathrm{mM}$ phenylthiourea (PTU), using $10 \mu \mathrm{l}$ per organism. Homogenates were centrifuged at $15,000 \mathrm{~g}$ for 10 minutes and aliquots of the supernatant stored at $-20^{\circ} \mathrm{C}$.

Subcellular fractions of $D$. melanogaster were prepared by the method of Dr. V. K. Walker (pers. comm.) from duplicate, staged cultures. Fresh flies were homogenised at $4^{\circ} \mathrm{C}$ using a loose fitting teflon-glass homogeniser in $50 \mathrm{mM}$ Tris- $\mathrm{HCl}$, $\mathrm{pH} 7.5$ containing $25 \mathrm{mM} \mathrm{KCl}, 5 \mathrm{mM} \mathrm{Mg}$ acetate, $350 \mathrm{mM}$ sucrose, $0.5 \mathrm{mM}$ PTU, and $1 \mathrm{mM}$ dithiothrietol. The homogenate was centrifuged at $80 \mathrm{~g}$ for 15 minutes to remove debris; $1,500 \mathrm{~g}$ for 10 minutes to pellet nuclei; $17,000 \mathrm{~g}$ for 20 minutes to pellet mitochondria; and $120,000 \mathrm{~g}$ for 60 minutes to pellet microsomes. The nuclear and mitochondrial pellets were washed by resuspension in the homogenising buffer and recentrifuged at $1,500 \mathrm{~g}$ and $17,000 \mathrm{~g}$, respectively. The microsomal pellet was rinsed with homogenising buffer. All pellets were resuspended in homogenising buffer and stored at $-20^{\circ} \mathrm{C}$ prior to use. No attempt was made to solubilise the subcellular fractions other than the disruption that would occur by one freeze-thaw cycle. 


\subsubsection{JHE Assay, Electrophoretic and Protein Detection Procedures}

\subsubsection{Protein Determination}

Protein concentrations were determined by the method of Bradford (1976) using the Biorad Protein Assay kit, except for native PAGE experiments for which the method of Markwell et al. (1978) was used. The latter reaction was performed in a total volume of $215 \mu \mathrm{l}$ and the absorbance was measured at $670 \mathrm{~nm}$. Bovine serum albumin (BSA) was used as a standard in both assays.

\subsubsection{Native Polyacrylamide Gel Electrophoresis (PAGE)}

The non-denaturing discontinuous gel system of Vernick et al. (1988) was used as described by Healy et al. (1991). Triton X-100 was omitted from the gels, except where indicated, because it has been reported to inhibit JHE activity in some insects (Hammock, 1985). Gels of $1 \mathrm{~mm}$ thickness were used to ensure rapid diffusion of substrates and inhibitors into the gel. Ester hydrolysis of naphthylacetate was detected using either $5.4 \times 10^{-4} \mathrm{M} \alpha$ - and $\beta$-naphthylacetate or $5.4 \times 10^{-4} \mathrm{M} \beta$-naphthylacetate alone. After visualisation of the esterases, gels were fixed in $40 \%$ methanol, $10 \%$ acetic acid, $3 \%$ glycerol solution and dried on paper under vacuum.

The sensitivity of esterases to the inhibitors diisopropylfluorophosphate (DFP; Sigma) and octylthiotrifluoropropanone (OTFP; a gift from the laboratory of Dr. B. D. Hammock) was assessed by including $10^{-4} \mathrm{M}$ solutions of these inhibitors in the washing and staining buffers.

\subsubsection{Kinetic Experiments Using Native PAGE}

Kinetic experiments to test whether JH inhibits naphthylacetate hydrolysis by esterases of $D$. melanogaster were performed as described by Rauschenbach et al. (1987), with some modifications. Aliquots of pupal homogenates (100 $\mu \mathrm{g}$ protein) containing the anodal esterases were fractionated by electrophoresis, as above. Triplicates of excised gel lanes were added to $10 \mathrm{ml}$ of a vigorously mixed staining solution containing JHIII (Sigma, at a final concentration of $0,2.5,5.0$ or $7.5 \times 10^{-4} \mathrm{M}$ added in $0.5 \mathrm{ml}$ ethanol) and B-naphthylacetate (at $0.8,1.3,2.7,5.4$ or $8.1 \times 10^{-4} \mathrm{M}$ ). Initial rates of reaction were determined by stopping one reaction in each triplicate set after 10,20 , or 30 minutes. The relative intensity of staining was determined by measuring the reflectance of individual esterase bands in dried gels at $520 \mathrm{~nm}$ using a Shimadzu CS-930 scanning densitometer. The data for zero JH allowed the calculation of the $\mathrm{K}_{\mathrm{M}}$ of EST20 for B-naphthylacetate. In order to determine the specificity of any inhibition of naphthylacetate hydrolysis by $\mathrm{JH}$, olive oil $(0-200 \mu \mathrm{l})$ was substituted for the JHIII.

A similar experiment was performed using a $D$. virilis pupal homogenate, except that JHI (Sigma) was used in place of JHIII. Two JHI concentrations $\left(10^{-4} \mathrm{M}\right.$ and $\left.5 \times 10^{-4} \mathrm{M}\right)$ and two amounts of olive oil $(2 \mu \mathrm{l}$ and $25 \mu \mathrm{l})$ were tested for inhibitition of $B$-naphthylacetate $\left(2.7 \times 10^{-4} \mathrm{M}\right)$ hydrolysis by the p-esterase.

\subsubsection{Isolation of JHE Activity After Native PAGE}

In order to determine whether JHE activity was associated with any of the naphthylacetate hydrolysing esterases, JHE activity was assayed directly from gel slices after native PAGE. Homogenates of $0-12$ hour pupae ( $1 \mathrm{mg}$ protein) were fractionated by native PAGE as described above. Each lane was sliced into at least 12 pieces and the 
JHE extracted from each gel slice by homogenisation in $1 \mathrm{ml}$ of $100 \mathrm{mM}$ sodium phosphate buffer, $\mathrm{pH} 8.0$. The resulting slurry was centrifuged at $10,000 \mathrm{~g}$ for 10 minutes at $4^{\circ} \mathrm{C}$ and the supernatant stored at $-20^{\circ} \mathrm{C}$ before being subject to the JHE partition assay described below.

\subsubsection{Thin Layer Chromatography to Identify JH Hydrolytic Products}

Thin layer chromatography (TLC) was used qualitatively to detect the products of $\mathrm{JH}$ metabolism generated by various life stages and subcellular fractions of $D$. melanogaster and $D$. virilis. The TLC system used was one of several described by Ottea et al. (1988). Enzyme samples (15-150 $\mu \mathrm{g}$ protein) were incubated at $30^{\circ} \mathrm{C}$ with $\left[10-{ }^{3} \mathrm{H}\right]-\mathrm{JHIII}(5 \mu \mathrm{M}, 4200 \mathrm{~Bq}$; NEN) in $100 \mathrm{mM}$ sodium phosphate buffer, $\mathrm{pH} 8.0$ at a final volume of $100 \mu \mathrm{l}$. JH and its products were extracted three times $\left(>96 \%{ }^{3} \mathrm{H}\right.$ recovered) with ethylacetate and separated on plastic-backed silica-60 A254 TLC plates (Merck, $70 \times 80 \mathrm{~mm}$ ), using hexane/ethylacetate (7:4) as the solvent system. The developed plates were sprayed with scintillant $(0.4 \% \mathrm{w} / \mathrm{v} 2,5$-diphenyloxazole (NEN) in 1-metnaphthalene) and air dried for 30 minutes. The locations of ${ }^{3} \mathrm{H}-\mathrm{JH}$ and its products were determined by exposure to X-ray film (Fuji XR100) for 20-40 hours (Shapiro et al., 1986). The relative mobilities (Rfs) for JH-diol, JH-acid, JHIII and n-butaneboronic acid (BBA) derivatised JH-diol (see below) were 0.11, 0.22, 0.50, and 0.61 respectively, which are in close agreement with the values obtained by Ottea $e t$ al. (1988) for this system.

Derivatising agents (BBA and diazomethane) and modification of the solvent system were used to confirm the identity of the products of $\mathrm{JH}$ metabolism (Hammock and Roe, 1985; Ottea et al., 1988). BBA reacts with vicinal diols and thereby increases the mobility of JH-diol and JH-acid-diol during TLC. Diazomethane reacts with carboxylic acids to form the corresponding methyl ester and thereby converts JH-acid and $\mathrm{JH}$-acid-diol to $\mathrm{JH}$ and $\mathrm{JH}$-diol, respectively. The predominant products of $\mathrm{JH}$ metabolism were found to be $\mathrm{JH}$-acid and $\mathrm{JH}$-diol, which permits the simultaneous determination of JHE and JHEH by the partition assay described below.

\subsubsection{Partition Assay of JH Hydrolytic Activities}

The JH hydrolytic activities due to JHE and JHEH were measured simultaneously and quantitatively by a simple modification of the commonly used radiometric partition assay of Hammock and Roe (1985). This assay was performed as described except that the buffer $\mathrm{pH}$ was increased to 8.0 and the volumes of the substrate solution added and samples taken were 2 and $100 \mu \mathrm{l}$, respectively. pH 8.0 is near optimal both for dipteran JHEHs which have very broad $\mathrm{pH}$ optima (Yu and Terriere, 1978b; Harshman et al., 1991), and for D. melanogaster JHE (determined below). One half of the reaction was stopped in the usual manner. The other half was treated likewise except that $2 \mathrm{mM} \mathrm{BBA}$ (final conc.) was included. In this assay unreacted $\mathrm{JH}$ is extracted into an organic phase whereas all the $\mathrm{JH}$-acid remains in the aqueous phase.

The percentage of JH-diol retained in the aqueous phase of the standard assay was determined in the following experiment. ${ }^{3} \mathrm{H}-\mathrm{JH}$-diol was generated using homogenates of 90 hour larvae. The reaction was partitioned or extracted in ethylacetate three times, as above, to recover the total $\mathrm{JH}$-diol generated. JH-diol from both the organic phase of the partition and the entire reaction were separated from $\mathrm{JH}$ and other metabolites by TLC. JH-diol was quantified by liquid scintillation counting of the 
appropriate regions of the TLC plate. $56 \pm 5 \%$ (SE, of 7 determinations) of the JH-diol was found in the aqueous phase, which is in good agreement with the previously determined value of $54.1 \pm 2 \%$ S.D. (Share and Roe, 1988). Following derivatisation by BBA, all the JH-diol was found in the organic phase. The half reaction with BBA thus contains only $\mathrm{JH}$-acid in the aqueous phase, whereas the half reaction without BBA contains the $\mathrm{JH}$-acid plus $56 \%$ of the $\mathrm{JH}$-diol in the aqueous phase. JHE activity is calculated from the radioactivity in the aqueous phase with BBA and the JHEH activity is calculated from the difference between samples with and without BBA. JHE activity was linear until $64 \%$ of the JH was consumed, whereas JHEH activity was linear until $33 \%$ of the $\mathrm{JH}$ was consumed.

\subsection{3 $\mathrm{pH}$, Isoelectric and Inhibition Properties of JHE}

\subsubsection{1 $\mathrm{pH}$ Profile of JHE Activity}

Homogenates of $D$. melanogaster pupae (mixed ages, 1 pupa per $10 \mu \mathrm{l}$ ) were prepared as described previously except that the homogenisation buffer was $10 \mathrm{mM}$ sodium phosphate, $\mathrm{pH} 6.8$. Aliquots of homogenate $(20 \mu \mathrm{l})$, or water controls, were added to $180 \mu \mathrm{l}$ of a $100 \mathrm{mM}$ buffer (see below) at the experimental $\mathrm{pH}$. Hydrolytic activity was determined using the partition assay (Section 2.2.2.6) except that no BBA was used. The ammonium hydroxide in the stop solution (Hammock and Roe, 1985) is sufficient to exceed the capacity of a $100 \mathrm{mM}$ buffer. The experimental buffers were citric acid-phosphate (7 buffers, $\mathrm{pH}$ 4.0-7.0), Imidazole- $\mathrm{HCl}$ (4 buffers, $\mathrm{pH}$ 6.6-8.0), and Tris- $\mathrm{HCl}$ (4 buffers, $\mathrm{pH} 7.6-9.0$ ).

\subsubsection{Isoelectric Focusing}

Isoelectric focusing (IEF) was performed in agarose in order to facilitate the recovery of $\mathrm{JH}$ hydrolysing enzymes from the gel. IEF gels containing $1 \% \mathrm{w} / \mathrm{v}$ agarose-IEF (Pharmacia), 12\% w/v sorbitol and $6.3 \% \mathrm{v} / \mathrm{v}$ ampholyte solution (Pharmalyte $\mathrm{pH} 3-10$ or $\mathrm{pH} 4-6.5$, Pharmacia) were cast $1 \mathrm{~mm}$ thick on $65 \times 145 \mathrm{~mm}$ plastic sheets (GelBond, Pharmacia). 12 hour pupae were homogenised in water (10 per $100 \mu \mathrm{l})$ with a few crystals of PTU. This homogenate $(30 \mu \mathrm{l})$ or isoelectric point marker proteins (Broad pI or Low pI Calibration Kit, Pharmacia) were applied to the gel on filter paper wicks which were removed after about 45 minutes of focussing. Homogenates were focussed at $24^{\circ} \mathrm{C}$ using the Pharmacia Flat Bed Electrophoresis Apparatus FBE3000 for a total of 90 minutes at 8 watts constant power (approximately 1,500 volt-hours). After focussing, the lane containing marker proteins was removed and stained using Coomassie Brilliant Blue R-250. Lanes containing fractionated homogenate were tested for $\mathrm{JH}$ hydrolysing activity. When using broad range ampholytes the lanes were cut into $5 \mathrm{~mm}$ slices. When using narrow range ampholytes the lane was sliced lengthways: one part was incubated with $\alpha$ - and $\beta$-naphthylacetate to detect esterase activity as described in section 2.2.2.2; the other was sliced into $5 \mathrm{~mm}$ slices or $2 \mathrm{~mm}$ slices in the region of the gel expected to contain JHE. JH hydrolysing activities were extracted from gel slices using a motor-driven pestle in $200 \mu \mathrm{l}$ of 100 $\mathrm{mM}$ sodium phosphate buffer, $\mathrm{pH}$ 8.0. The resultant slurry was centrifuged and the supernatant assayed for $\mathrm{JH}$ hydrolysis using the partition assay (Section 2.2.2.6). 


\subsubsection{Effect of Inhibitors on JHE}

Homogenates of 12 hour pupae ( $15 \mu \mathrm{g}$ total protein) were incubated with solutions of the esterase inhibitors DFP and OTFP $\left(10^{-4}\right.$ and $\left.10^{-5} \mathrm{M}\right)$ in assay buffer for 10 minutes. The incubated samples and untreated controls were then assayed by the partition method (Section 2.2.2.6). DFP was stored for five days as a $100 \mu \mathrm{M}$ solution in ethanol at $4{ }^{\circ} \mathrm{C}$ prior to use. Fresh DFP from some sources has been reported to contain a potent but unstable inhibitor of lepidopteran JHEs. Storage in ethanol solution destroys the inhibitor without affecting the potency of the DFP against other esterases (Hanzlik and Hammock, 1987). The ethanol-stored DFP (1 mM) used in this study had no effect on the JHE activity in the haemolymph of the lepidopteran, G. mellonella, previously shown to be insensitive to DFP (Rudnicka and Kochman, 1984).

\subsection{Results}

\subsubsection{Comparison of the Anodal Esterases of Drosophila melanogaster and Drosophila virilis}

\subsubsection{Pattern of Expression of the Anodal Esterases}

Pupae of $D$. melanogaster and $D$. virilis exhibit very similar electrophoretic profiles of naphthylacetate hydrolysing esterases (Fig. 2.1), suggesting that homologous esterases of these two species could be readily identified. The p-esterase of $D$. virilis has been characterised as preferentially hydrolysing B-naphthylacatate over $\alpha$-naphthylacetate, having high mobility on native PAGE, responding to increased larval crowding with reduced expression in pupae and having predominantly pupal expression (Rauschenbach et al., 1977). Of the three anodal esterases of D. melanogaster which preferentially hydrolyse ß-naphthylacetate (EST19, EST20 and EST21; Healy et al., 1991), the Rf of EST20 (0.91) corresponds most closely to that of the p-esterase of D. virilis (0.91). EST19 and EST21 were not present in the samples used for this experiment but are shown in figures 2.2,2.3 and 2.4.

Increasing the culture density consistently decreased the expression of EST20 in D. melanogaster (Fig. 2.1). In contrast, other esterases either responded inconsistently or were unaffected by culture density. Whereas low culture density was usually sufficient to obtain EST20 activity in pupae, it was often not sufficient to ensure expression of EST19 or EST21. Although three replicate experiments revealed some variation in the extent of crowding required to reduce EST20 expression, vials seeded with 30 to 100 eggs showed decreased levels of expression in all three experiments. EST20 thus resembles the p-esterase of $D$. virilis in respect of crowding effects.

The developmental profiles of EST19, EST20 and EST21 are shown in figure 2.2. Although these esterases show some expression in larvae and adults, they are predominantly expressed in pupae, as is the p-esterase of $D$. virilis (Rauschenbach et al., 1977).

(this study and Healy et al., 1991)

At the end of pupal life some D. melanogaster esterases including EST20, EST2 1 and the $\alpha$-naphthylacetate hydrolysing EST22 remain in the discarded pupal case, whereas others are found in the newly emerged adult. In contrast, in $D$. virilis the p-esterase is retained in newly emerged adults (Rauschenbach et al., 1977), although two other esterases are found in the pupal case (Fig. 2.3). 


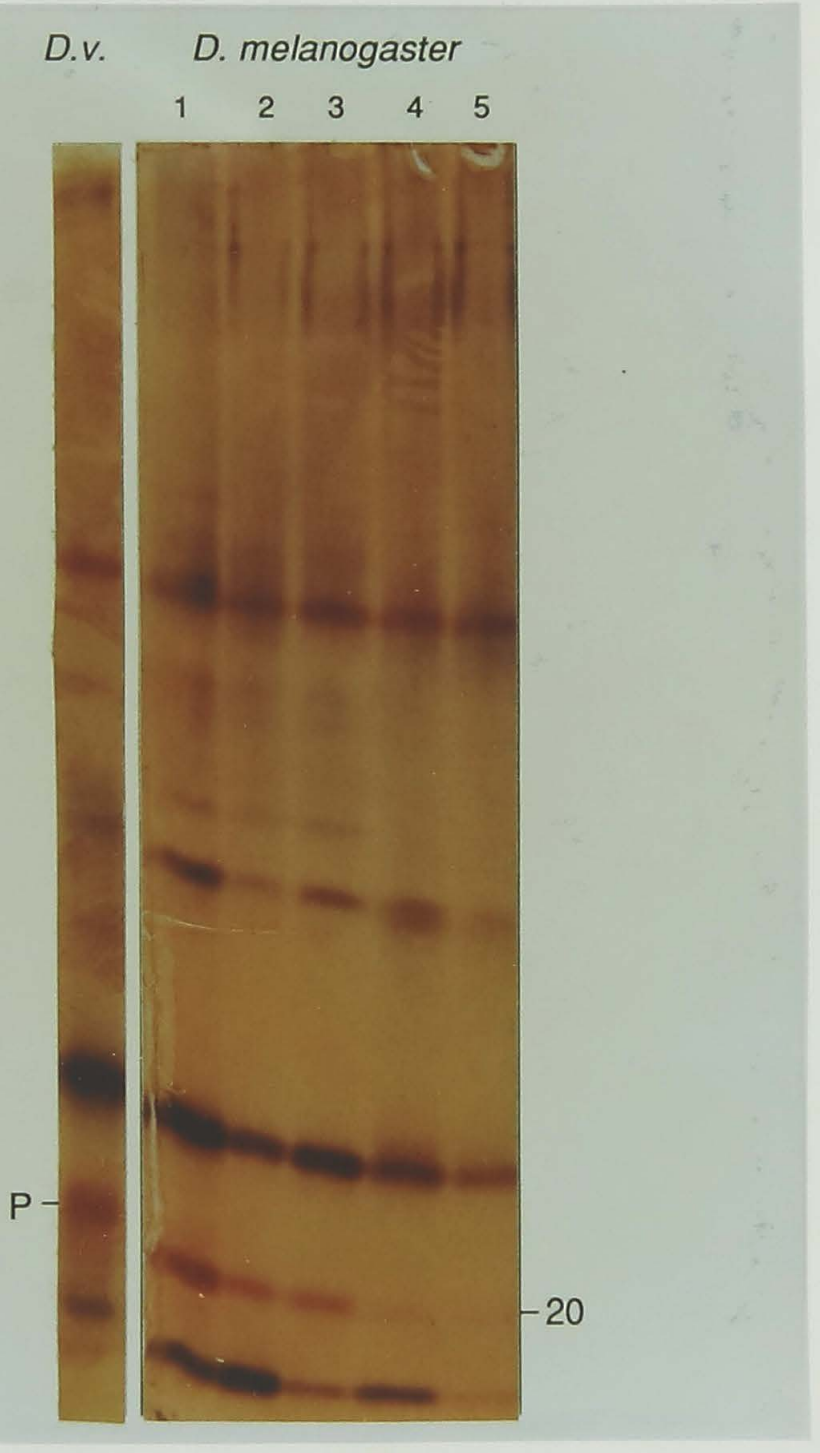

Figure 2.1 Comparison Between the Esterases of $D$. virilis and D. melanogaster Pupae Showing the Effect of Larval Culture Density on EST20 Expression.

Esterases were separated by native PAGE and detected using the in vitro substrates $\alpha$ - and $\beta$-naphthylacetate. Brown and red bands indicate esterases which preferentially hydrolyse $\alpha$ - and $\beta$-naphthylacetate, respectively. The left lane (D.v) shows the esterases of $D$. virilis pupae $(200 \mu \mathrm{g}$ protein) aligned with the esterases of $D$. melanogaster. The locations of the $D$. virilis $\mathrm{p}$-esterase $(\mathrm{P})$ and the $D$. melanogaster esterase $20(20)$ are indicated. The remaining lanes show the effect of larval culture density on esterase activities in pupal homogenates $(200 \mu \mathrm{g}$ protein) of D. melanogaster. Organisms were cultured as described in section 2.2.1.1. For lanes 1 to 5 , vials were seeded with $10,30,60,100$ or 200 eggs, respectively. 


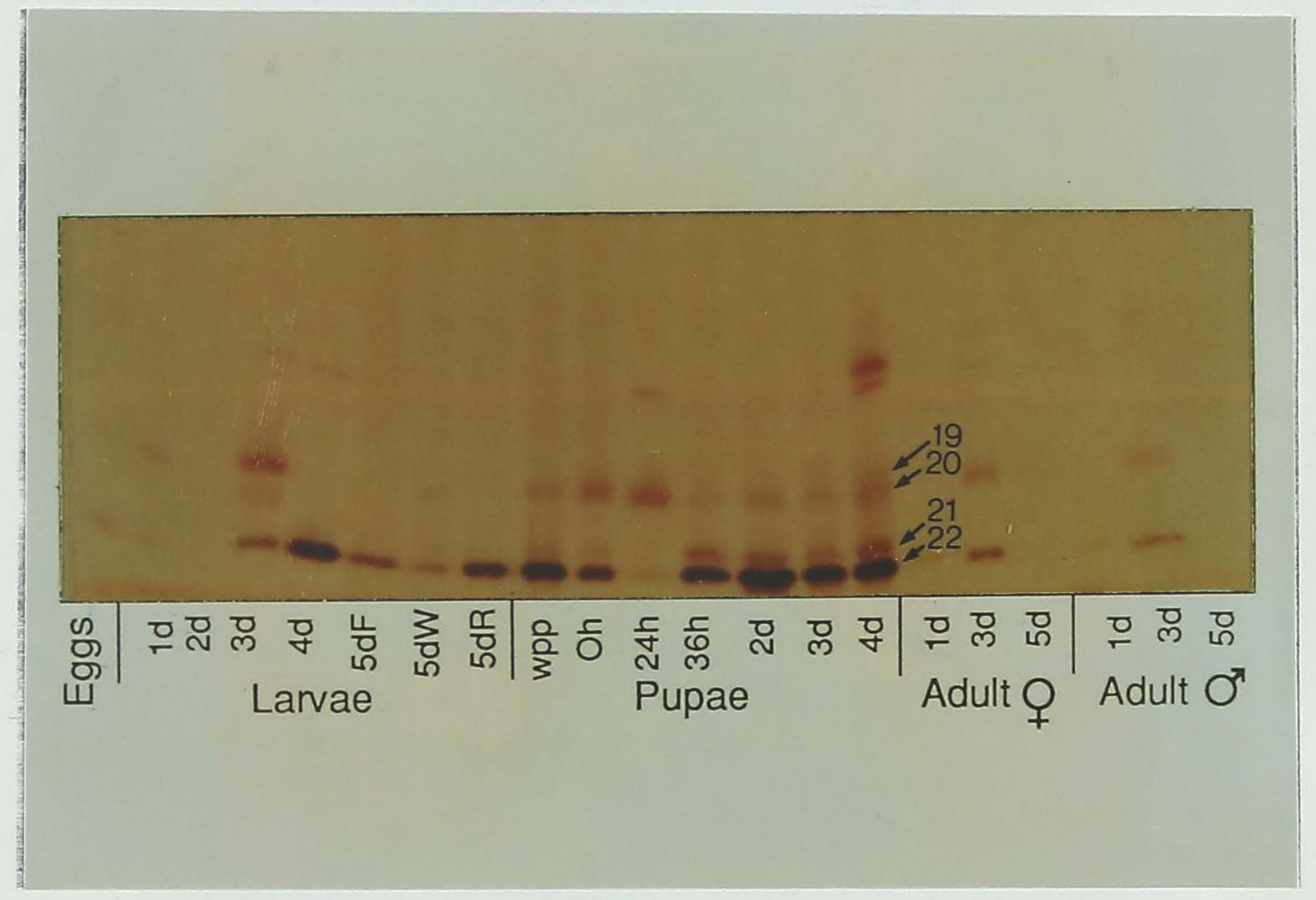

Figure 2.2 Developmental Profile of Anodal Esterases of D. melanogaster.

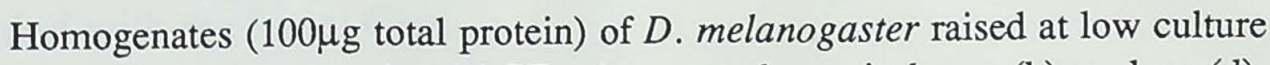
density were separated by native PAGE. Ages are shown in hours (h) or days (d). Five day larvae were feeding $(\mathrm{F})$, wandering $(\mathrm{W})$, or resting $(\mathrm{R})$. Esterase activity was detected using $\alpha$ - and $\beta$-naphthylacetate. The anodal region only is shown (Rf 0.6-1.0). The white prepupal stage is indicated (wpp). 


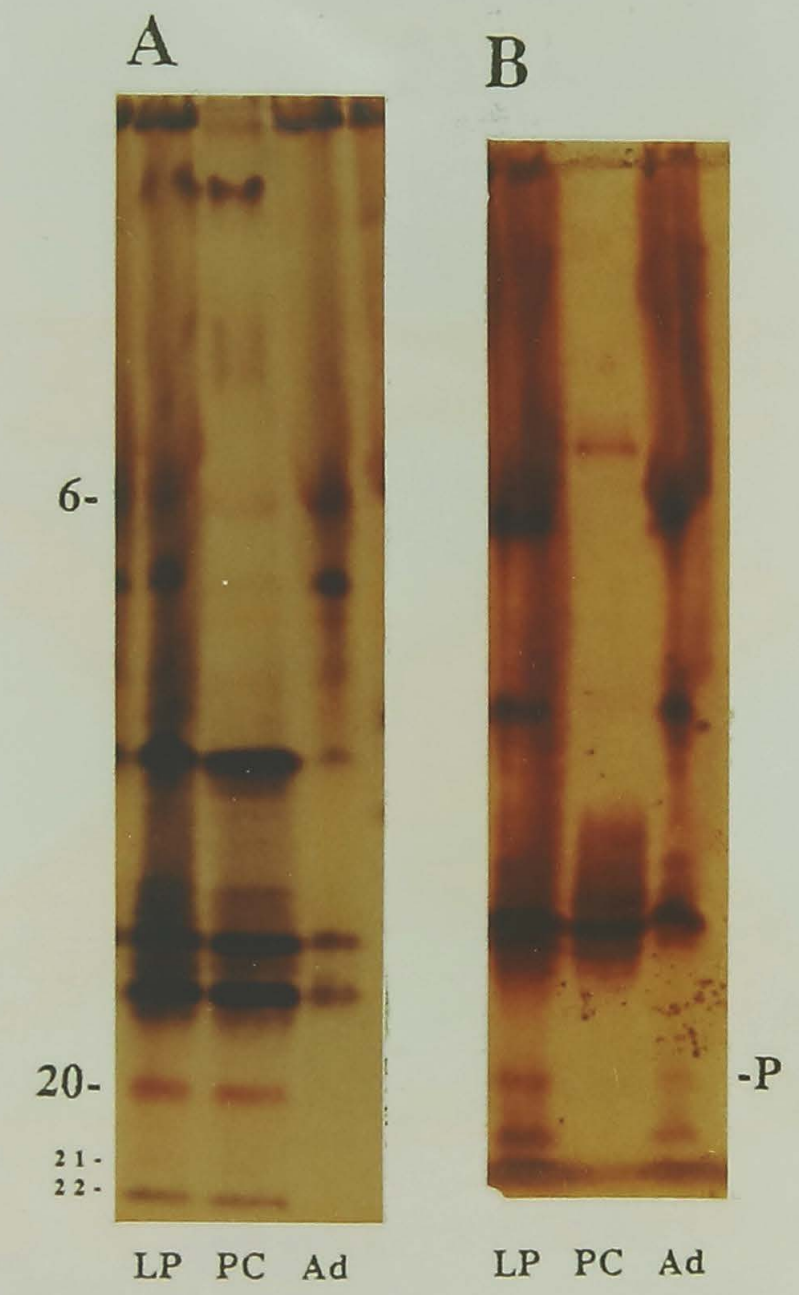

Figure 2.3 Comparison of Esterases Retained After Adult Ecdysis or discarded with the Pupal Case

Prepupae were staged to within two hours then incubated until about half the adults had emerged. The remaining pharate adults, the discarded pupal cases and the newly emerged adults were collected, homogenised, separated by native PAGE and stained for esterase activity as described in sections 2.2.1 and 2.2.2.2.

Panel A shows esterases separated from D. melanogaster and panel B shows esterases separated from $D$. virilis. LP indicates late pupae (pharate adults), PC indicates discarded pupal cases, and Ad indicates newly eclosed adults. Two organism/pupal case equivalents were loaded in each lane. Thus, the esterases present in LP represent the sum of esterases present in PC and Ad. The locations of EST6, EST20, EST21, EST22 and the p-esterase are indicated 


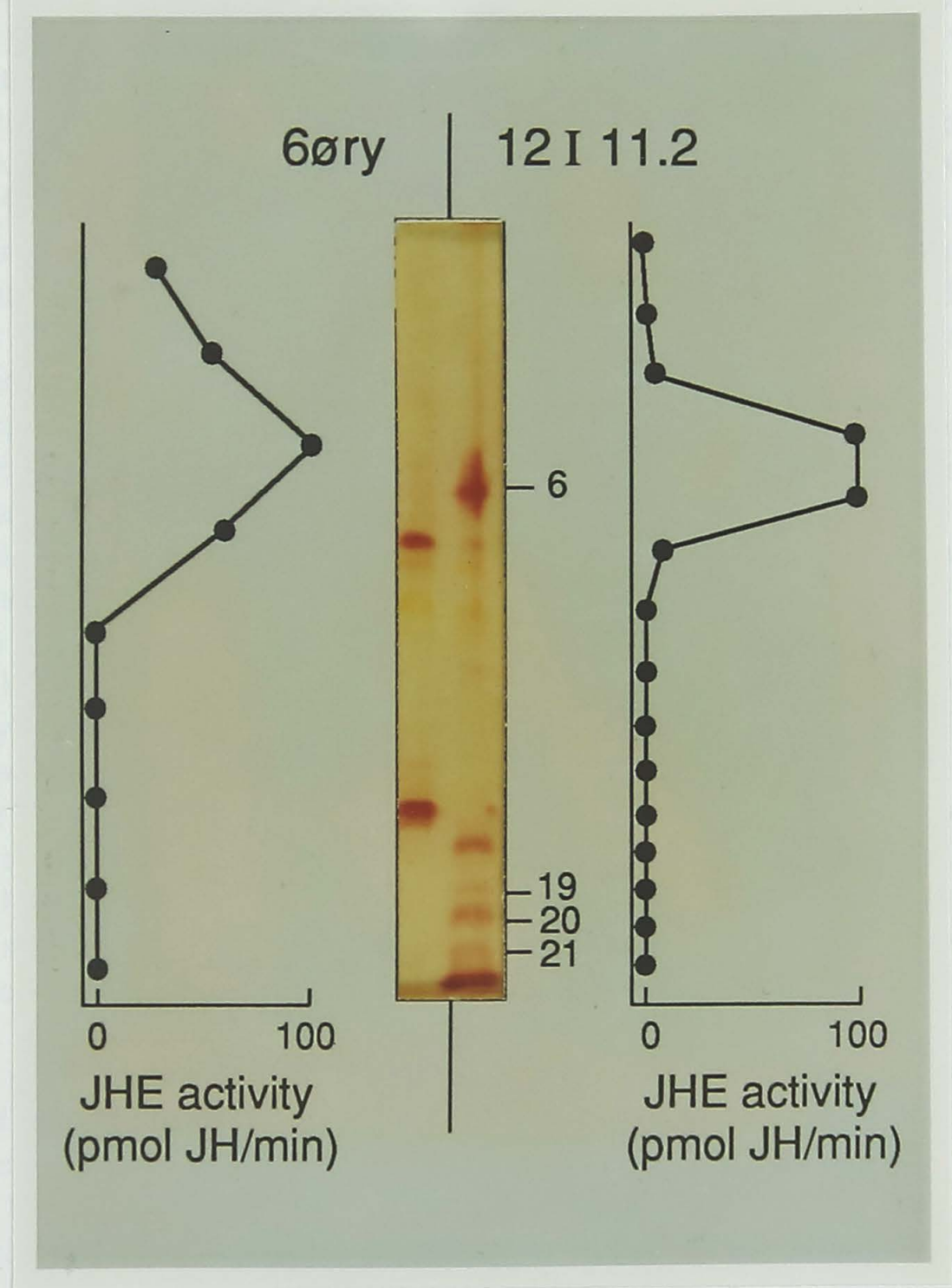

Figure 2.4 JHE Activity Isolated from Polyacrylamide Gel Slices.

Homogenates of D. melanogaster pupae (1 mg protein), strains Est $6^{\text {null }} r y$ (6øry) and 12I11.2, were separated by native PAGE and the JHE activity extracted from gel slices was measured using the partition assay method. $100 \mu \mathrm{g}$ samples of the same homogenates were separated by native PAGE and esterases were detected using B-naphthylacetate. 


\subsubsection{Biochemical Properties of the Anodal Esterases}

The $D$. melanogaster naphthylacetate hydrolysing esterases most sensitive to inhibition by $10^{-4}$ M OTFP are EST19, EST20 and EST21, although these enzymes are resistant to $10^{-4}$ M DFP (Healy et al., 1991). Under the same conditions I find that the D. virilis p-esterase is resistant to DFP (as reported by Rauschenbach et al., 1987) but is completely inhibited by OTFP (not tested by Rauschenbach et al., 1987).

The $\mathrm{K}_{\mathrm{M}}$ of $D$. melanogaster EST20 for B-naphthylacetate hydrolysis is $2.9 \pm 0.6 \times 10^{-4} \mathrm{M}$ (SE from 4 determinations), which is similar to the value of $1.0 \times 10^{-4} \mathrm{M}$ determined for the $D$. virilis p-esterase (Rauschenbach et al., 1987). The intensity of staining of other esterases was unaffected by the change in naphthylacetate concentration in the conditions of this experiment, indicating that these esterases are operating at their maximum velocity at the concentrations of naphthylacetate used.

Rauschenbach et al. (1987) reported that naphthylacetate hydrolysis by the p-esterase of $D$. virilis is competitively inhibited by $\mathrm{JH}$. However, I was unable to demonstrate competitive inhibition of $B$-naphthylacetate hydrolysis by soluble amounts of $\mathrm{JH}$ using either D. melanogaster or D. virilis homogenates. Hydrolysis of naphthylacetate by EST 20 and EST 21 of D. melanogaster (as measured by staining intensity) was reduced by $15-55 \%$ in the presence of insoluble quantities of JHIII, although hydrolysis by other esterases was not affected. This effect occurred when the B-naphthylacetate concentration was 1.3-5.4 x $10^{-4} \mathrm{M}$ and the concentration of JHIII, if soluble, would have been $2.5-7.5 \times 10^{-4} \mathrm{M}$.

In order to test the specificity of this reduction in hydrolysis, a substance expected to have similar physical properties to $\mathrm{JH}$, namely olive oil, was substituted for JH. $25 \mu \mathrm{l}$ olive oil reduced EST20 hydrolysis by $18 \%$, and $100 \mu \mathrm{l}$ olive oil reduced it by $50 \%$, suggesting that the effect is not $\mathrm{JH}$-specific. Hydrolysis by other esterases was reduced to a lesser extent and $5 \mu \mathrm{l}$ or less olive oil had no effect on any of the esterases.

A similar experiment was performed with the p-esterase of $D$. virilis using JHI instead of JHIII because JHI had been claimed to cause competitive inhibition of the p-esterase (Rauschenbach et al., 1987). Neither $10^{-4} \mathrm{M}$ JHI nor $2 \mu \mathrm{l}$ olive oil had any effect on hydrolysis by the p-esterase. However, both $5 \times 10^{-4} \mathrm{M} \mathrm{JHI}$ and $25 \mu \mathrm{l}$ olive oil markedly reduced hydrolysis by the p-esterase in comparison to the other esterases, suggesting that the effect of JHI on hydrolysis by the p-esterase is not specific.

\subsubsection{Characterisation of JH Hydrolysis}

\subsubsection{Localisation of JHE After Native PAGE}

In view of the non-specific inhibition of naphthylacetate hydrolysis by $\mathrm{JH}$, proteins were extracted from gels after native PAGE of pupal homogenates and assayed for JHE directly using the partition assay. For D. melanogaster no activity was recovered from the region of the gels corresponding to the anodal esterases, but about $70 \%$ of the applied JHE activity was recovered (with or without $0.1 \%$ Triton X-100) at an Rf of 0.36 (Fig. 2.4). As the Rf of D. melanogaster JHE is close to that of the ß-naphthylacetate staining enzyme EST6 (0.34), an Est6 $6^{\text {null }}$ strain was used to determine whether JHE and EST6 could be separated. JHE activity from the Est 6 null strain was recovered at the same Rf as in the 12I11.2 strain and the level of JHE activity 
was similar. Only a trace of naphthylacetate hydrolysis was detected in the region of the gel from which JHE was recovered (Fig. 2.4).

As described above, it is possible to alter the culture conditions of larvae in order to produce pupae either expressing or lacking the anodal esterases. I find that the level of JHE activity in homogenates of pupae of the same age is similar whether anodal esterases are present or absent, for both D. melanogaster and D. virilis .

\subsubsection{Developmental Profile of JHIII-Hydrolysis}

Two experiments were performed to identify the JHIII hydrolysing enzymes in D. melanogaster at different developmental stages. The first used TLC qualitatively to identify the enzymes responsible for JH hydrolysis at three developmental stages (Fig. 2.5). In ninety hour larvae, JH-diol was found together with a trace of JH-acid, indicating that $\mathrm{JHEH}$ is the predominant $\mathrm{JH}$ hydrolytic enzyme at this stage. In twelve hour pupae, $\mathrm{JH}$-acid was found together with traces of $\mathrm{JH}$-diol (and sometimes a trace of an unidentified metabolite which remained near the origin), indicating that JHE is the predominant $\mathrm{JH}$ hydrolytic enzyme at this stage. In five day adult females, similar amounts of $\mathrm{JH}$-acid and $\mathrm{JH}$-diol were found, indicating the presence of both $\mathrm{JHE}$ and $\mathrm{JHEH}$. JH-acid-diol was not found at this stage, indicating that JH-diol is not a good substrate for JHE, and that $\mathrm{JH}$-acid is not a good substrate for the JHEH, a conclusion also reached by Ottea et al. (1988). Also, in twelve hour pupae of $D$. virilis, JH-acid was found together with a trace of $\mathrm{JH}$-diol, indicating that, as with D. melanogaster, $\mathrm{JHE}$ is the predominant $\mathrm{JH}$ hydrolytic enzyme at this stage.

A more complete quantitative developmental profile of JH hydrolytic activities in D. melanogaster was obtained using the partition assay. Two periods of high activity were revealed (Fig. 2.6). The first occurs in 70 to 92 hours old larvae and is predominantly due to JHEH ( $86 \%$ of total hydrolysis). Late in the final larval instar both JHE and JHEH activities fall, reaching a minimum in white prepupae (stage P1 of Bainbridge and Bownes, 1981) and zero hour pupae (stage P3-4(i)). The second and major peak of JH hydrolytic activity is due mainly to JHE (also $86 \%$ of total hydrolysis) and occurs in 6 to 12 hour pupae. This is when pupal apolysis (the pupal moult, stage P4(ii)) occurs. After this time hydrolytic activity declines, reaching a low level by the end of the pupal stage. Adults of both sexes exhibit low (albeit underestimated in these results, see below) levels of JH hydrolytic activity, with JHE activity being similar in both males and females and remaining constant. JHEH activity rises slightly in older adults, about twice as much in females as males, which may be correlated with different roles of JH in sex-specific reproductive events (Koeppe et al., 1985).

A control experiment demonstrated that storage at $-20^{\circ} \mathrm{C}$ for 5 days reduces JHE activity in homogenates of fresh male and female adult flies by $52 \%$ and $55 \%$ respectively $(n=4, p<0.01)$. No further loss of adult JHE activity was observed after refreezing and a further 8 days of storage at $-20^{\circ} \mathrm{C}$. No significant losses $(n=4$, $\mathrm{p}>0.05$ ) in the activities of JHE or JHEH were found for homogenates of 90 hour old larvae, 12 hour old pupae, or for JHEH in adult flies assayed before and after freezing.

\subsubsection{Subcellular Localisation of JH Hydrolytic Activities}

Nuclear, mitochondrial, microsomal and soluble fractions were prepared from 90 hour larvae, 12 hour pupae and 5 day female adults of $D$. melanogaster and the levels of $\mathrm{JH}$ hydrolytic activities in each were determined using the partition assay (Table 2.1). 


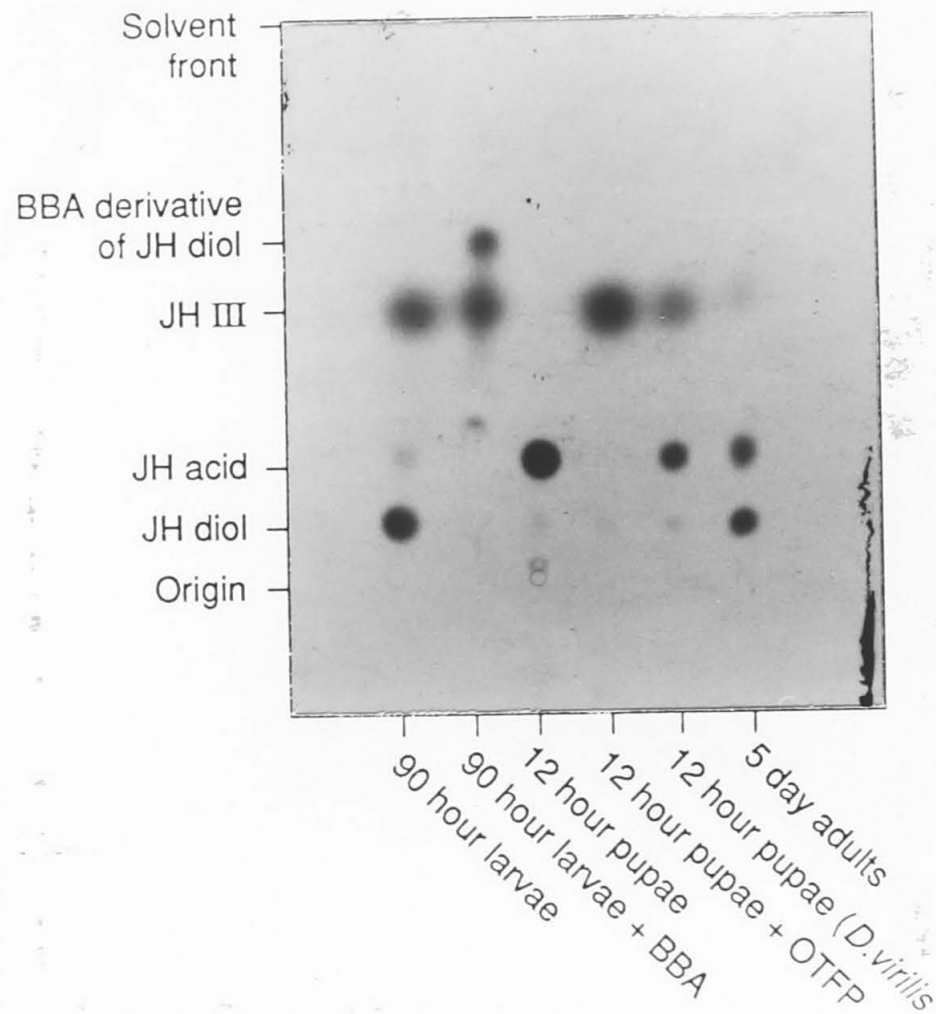

Figure 2.5 Thin Layer Chromatography of the Products of JHHydrolysis.

The products of JH-hydrolysis at defined developmental stages were visualised by TLC followed by fluorography, as described in section 2.2 .2 .5 . OTFP $\left(10^{-4} \mathrm{M}\right)$, where indicated, was added to the reaction mixture prior to the start of the incubation. OTFP removes all JHE activity, eliminating $\mathrm{JH}$-acid production. 1-butaneboronic acid (BBA), where indicated, was added to the reaction extract before it was applied to the TLC plate. The BBA derivative of JH-diol has an increased mobility. Results shown were obtained with $D$. melanogaster except where $D$. virilis is indicated. 


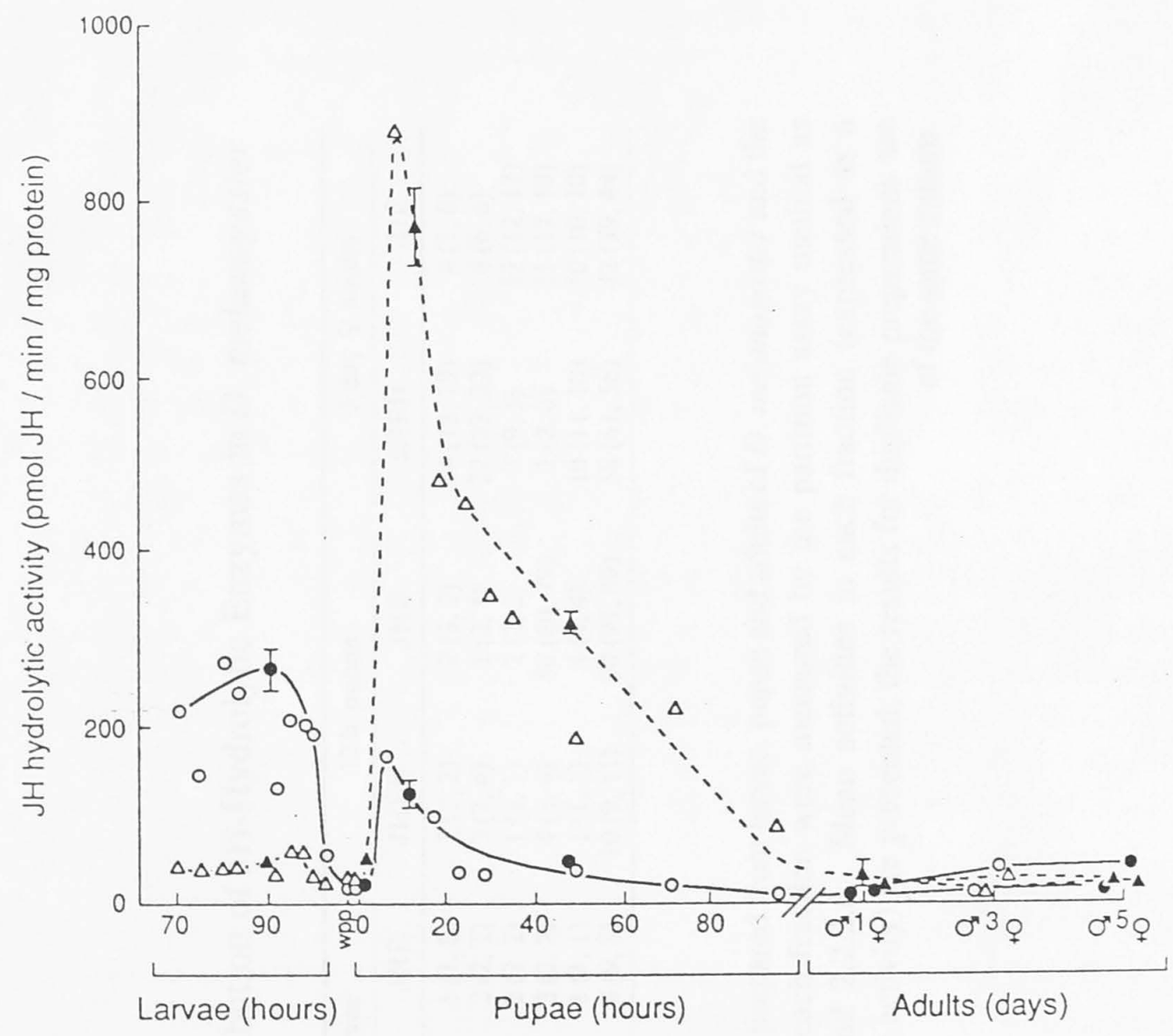

Figure 2.6 Developmental Profile of JHE and JHEH Activities in D. melanogaster.

Specific activities of JHE and JHEH were determined simultaneously for homogenates of $D$. melanogaster using the partition assay as described in section 2.2.2.6. Triangles indicate JHE activity and circles indicate JHEH activity. Solid symbols represent the mean result for four independent homogenates; standard errors are shown only where they exceed the size of the symbol. Open symbols represent single data points. The white prepuparium stage is indicated by "wpp". 
Table 2.1 Subcellular Distribution of JH-Hydrolytic Enzymes in D. melanogaster

\begin{tabular}{|c|c|c|c|c|c|c|}
\hline \multirow[b]{2}{*}{ Fraction } & \multicolumn{2}{|c|}{$90 \mathrm{~h}$ larvae } & \multicolumn{2}{|c|}{$12 \mathrm{~h}$ pupae } & \multicolumn{2}{|c|}{5 day $q$ adults } \\
\hline & JHEH & JHE & JHEH & JHE & JHEH & JHE \\
\hline Nuclear & $6(6,5)$ & $1(1,0)$ & $2(2,2)$ & $2(3,2)$ & $14(18,19)$ & $4(1,6)$ \\
\hline Mitochondrial & $50(47,52)$ & $2(2,2)$ & $3(2,4)$ & $1(2,1)$ & $27(32,22)$ & $6(6,6)$ \\
\hline Microsomal & $17(18,16)$ & $2(2,2)$ & $1(1,1)$ & $2(2,2)$ & $7(9,5)$ & $12(12,12)$ \\
\hline Soluble & $5(6,4)$ & $3(3,2)$ & $4(5,4)$ & $80(80,79)$ & $1(2,0)$ & $11(12,10)$ \\
\hline Washes & $14(14,14)$ & $1(1,1)$ & $1(1,1)$ & $4(4,4)$ & $10(11,10)$ & $10(10,10)$ \\
\hline Total & $92(91,92)$ & $8(9,8)$ & $10(9,11)$ & $90(91,89)$ & $58(61,56)$ & $42(39,44)$ \\
\hline
\end{tabular}

Subcellular fractions were prepared from larvae, pupae and adults of D. melanogaster and the activities of JHE and JHEH in each fraction were measured by the partition assay method as described in sections 2.2.1.2 and 2.2.2.6. Mean activities in each fraction (expressed as a percentage of total JH-hydrolytic activity) are presented; the results for duplicate preparations are given in parentheses.

of the same culture 
In larvae, $92 \%$ of the total JH hydrolytic activity was due to JHEH. JHEH in the mitochondrial and the microsomal fractions contributed $50 \%$ and $17 \%$, respectively, to the total JH hydrolytic activity recovered at this life stage. In pupae, $80 \%$ of the total $\mathrm{JH}$ hydrolytic activity recovered was JHE from the soluble fraction. In adults, JHE and JHEH contributed more equally to total JH hydrolytic activity; the highest levels of JHEH were found in the mitochondrial fraction (27\% of total hydrolysis) but JHE was distributed approximately equally between the microsomal and soluble fractions ( $12 \%$ and $11 \%$ of total hydrolysis, respectively).

\subsubsection{Biochemical Properties of JHE}

Varying the hydrogen ion concentration of the assay solution over a range from $\mathrm{pH} 4.0$ to 9.0 revealed no buffer-specific effects on the activity of JHE from pupae of D. melanogaster. Below pH 6.0 considerable non-enzymic, acid hydrolysis of $\mathrm{JH}$ occurred. Over the $\mathrm{pH}$ range from 6.0 to 8.6 , JHE activity almost doubled, increasing linearly with increasing $\mathrm{pH}$. No further change in activity was observed at $\mathrm{pH} 9.0$. This result is very similar to the profile of activity variation with $\mathrm{pH}$ found for $D$. hydei JHE (Bisser and Emmerich, 1981).

After IEF ( $\mathrm{pH} 3-10)$, JH hydrolysis was observed between $\mathrm{pH} 5.2$ and $\mathrm{pH} 5.85$ for homogenates of 12 hour pupae of both D. melanogaster and D. virilis. Using

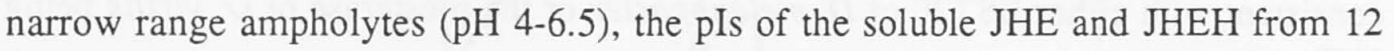
hour pupae of $D$. melanogaster were found to be $5.41 \pm 0.04$ and $6.25 \pm 0.13$ (SE, 3 determinations), respectively (Fig. 2.7). The $\mathrm{pI}$ of JHE from D. melanogaster coincides with an $\alpha$-naphthylacetate staining esterase not previously observed by native PAGE or IEF (Healy et al., 1991) and similarly the $\mathrm{pI}$ of JHE of D. virilis coincides with an esterase which preferentially hydrolyses $\alpha$-naphthylacetate over $\beta$ naphthylacetate.

The sensitivity of JHE to the esterase inhibitors OTFP and DFP was determined for homogenates of 12 hour pupae of both D. melanogaster and D. virilis. Greater than $95 \%$ of the JHE activity in homogenates from both species was removed by $10^{-5} \mathrm{M}$ OTFP. $10^{-4} \mathrm{M}$ DFP removed all JHE activity from $D$. melanogaster homogenates and $10^{-5} \mathrm{M}$ DFP removed $87 \pm 3 \%$ (SE, 3 determinations). $10^{-4}$ M DFP removed $94 \pm 2 \%$ JHE activity (SE, 4 determinations) from $D$. virilis homogenates and $10^{-5} \mathrm{M}$ DFP removed $50 \pm 5 \%$ (SE, 4 determinations).

\subsection{Discussion}

\subsubsection{Comparisons Among the Anodal Esterases and JHE}

\subsubsection{Comparison Between p-Esterase and EST20}

This study provides several lines of evidence suggesting that the p-esterase of $D$. virilis is homologous to EST20 of D. melanogaster. The two esterases have similar relative mobility on native PAGE, substrate preference, kinetics with $B$-naphthylacetate and sensitivity to the inhibitors DFP and OTFP. Both enzymes are predominantly expressed in pupae and respond to increased larval crowding with reduced pupal expression. The only property studied which differs between the two enzymes is their fate at eclosion, the p-esterase being retained in the adult whereas EST20 is discarded with the pupal case. The interpretation of homology between the p-esterase and EST20 
is supported by studies in other esterase gene/enzyme systems. In particular, the homologues of $D$. melanogaster EST6 identified initially on the basis of shared electrophoretic properties and substrate preferences have now been confirmed by genetic and molecular biological studies (Oakeshott et al., 1990).

Fewer data were collected for EST19 and EST21 of D. melanogaster, but they also share properties with the $D$. virilis p-esterase and anodal esterases in other Drosophila species. Shared properties include substrate preference, high mobility on native PAGE, predominantly pupal expression, retention in the pupal case at adult eclosion (EST21 only), resistance to DFP inhibition and a requirement for low crowding of larvae for expression (Berger and Canter, 1972; 1973; Osterbur and Steiner, 1979). The evolutionary relationship between the anodal esterases has not been studied but mapping of the encoding genes may resolve this question.

\subsubsection{Association Between p-Esterase/EST20 and JHE}

The claim that the $D$. virilis p-esterase is JHE was based largely on three observations: JHI appeared to competitively inhibit its hydrolysis of B-naphthylacetate; $\mathrm{JH}$ hydrolytic activity was only observed in homogenates with p-esterase activity; and its activity followed the developmental profile for JHE (Rauschenbach et al., 1987; 1991). However, as discussed below, my results on each of these points are not consistent with either EST20 of D. melanogaster or the p-esterase of $D$. virilis being JHE.

Considering first the issue of inhibition by $\mathrm{JH}$, this study did not reveal competitive inhibition by JH of any of the naphthylacetate hydrolysing enzymes of either Drosophila species. Large quantities of JH do reduce naphthylacetate hydrolysis by EST20 of $D$. melanogaster and the p-esterase of $D$. virilis. However, this effect is neither specific, since it can be duplicated using olive oil, nor competitive, since it only occurs with greater than soluble amounts of JH. The concentrations of JHI reported by Rauschenbach et al. (1987) to cause competitive inhibition of the $D$. virilis p-esterase (0.8-3.8 $\left.\times 10^{-4} \mathrm{M}\right)$ are higher than the aqueous solubility of $\mathrm{JH}\left(3-5 \times 10^{-5} \mathrm{M}\right.$; Pratt, 1975; Kramer and de Kort, 1976). I suggest that the reduction in staining of some esterases in the presence of insoluble JH may occur because naphthylacetate is partitioned between the undissolved $\mathrm{JH}$ and the aqueous phase, thereby reducing its aqueous concentration. Staining would be reduced only for those esterases which are hydrolysing naphthylacetate at less than their maximum rates at the lower concentration. This hypothesis is supported by the observation that the $\mathrm{K}_{\mathrm{MS}}$ of EST20 and p-esterase (2.4 $\times 10^{-4} \mathrm{M}$ and $1 \times 10^{-4} \mathrm{M}$ (Rauschenbach et al., 1987), respectively) are higher than for other Drosophila esterases which preferentially hydrolyse B-naphthylacetate; EST6 has a $\mathrm{K}_{\mathrm{M}}$ of about $3 \mu \mathrm{M}$ (Mane et al., 1983) and the $\mathrm{K}_{\mathrm{M}^{\mathrm{S}}}$ of most other Besterases of Drosophila species are also in the micromolar range (White et al., 1988). The $\mathrm{K}_{\mathrm{MS}}$ of EST20/p-esterase are therefore similar to the naphthylacetate concentration used for gel staining $\left(5 \times 10^{-4} \mathrm{M}\right)$, so any reduction in naphthylacetate concentration would be expected to reduce the rates of hydrolysis by these enzymes, while only large reductions in naphthylacetate concentration would be expected to reduce the rates of hydrolysis by the other B-naphthylacetate hydrolysing esterases. Thus, if $\mathrm{JH}$ and olive oil do dissolve some naphthylacetate, EST20 and p-esterase would be expected to exhibit the greatest reduction in staining intensity, as is observed.

My results also contrast with those of Rauschenbach et al. (1987) in respect of the reported congruence of JHE and the p-esterase/EST20 activities. Thus, I find that 


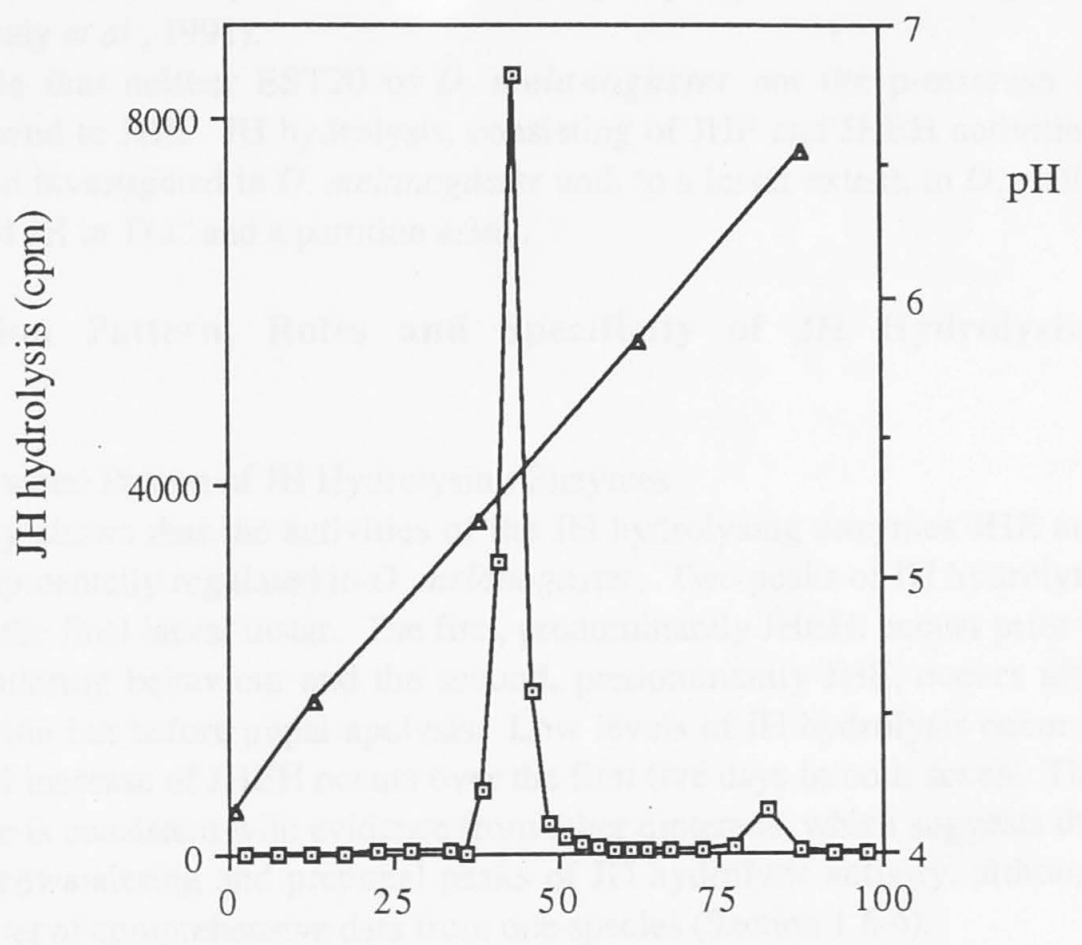

Distance from positive electrode (mm)

Figure 2.7 Isoelectric Focussing of JH Hydrolytic Enzymes

Homogenates of whole D. melanogaster pupae (three organism equivalents in $30 \mu \mathrm{l})$ and isoelectric point marker proteins (Low pI Calibration Kit, Pharmacia) were subjected to isoelectric focussing using narrow range ampholytes as described in section 2.2.3.2. This figure shows a typical experiment in which JHE focussed at $\mathrm{pH}$ 5.4 and JHEH focussed at $\mathrm{pH} 6.3$. 
JHE activity is similar in homogenates of $D$. virilis pupae with and without p-esterase activity and in homogenates of $D$. melanogaster pupae in which EST20 is present or absent. In addition, JHE in D. melanogaster does not have the same developmental profile as any of the esterases previously observed by naphthylacetate staining after native PAGE (Healy et al., 1991).

I conclude that neither EST20 of D. melanogaster nor the p-esterase of D. virilis correspond to JHE. JH hydrolysis, consisting of JHE and JHEH activities, has therefore been investigated in $D$. melanogaster and, to a lesser extent, in $D$. virilis using radiolabeled $\mathrm{JH}$ in TLC and a partition assay.

\subsubsection{Expression Pattern, Roles and Specificity of JH Hydrolysing Enzymes}

\subsubsection{Developmental Pattern of JH Hydrolysing Enzymes}

This study shows that the activities of the JH hydrolysing enzymes JHE and $\mathrm{JHEH}$ are developmentally regulated in D. melanogaster. Two peaks of JH hydrolytic activity occur in the final larval instar. The first, predominantly JHEH, occurs prior to the onset of wandering behaviour and the second, predominantly JHE, occurs after puparium formation but before pupal apolysis. Low levels of JH hydrolysis occur in adults but a small increase of JHEH occurs over the first five days in both sexes. This expression profile is consistent with evidence from other dipterans, which suggests that they also have prewandering and prepupal peaks of JH hydrolytic activity, although there is no other set of comprehensive data from one species (Section 1.6.4).

Although final instar larvae of both dipterans and lepidopterans exhibit prewandering and prepupal maxima of JH hydrolysis, the relative contributions of JHE and JHEH both within and between orders may be different. In D. melanogaster $\mathrm{JH}$ hydrolysis in prewandering larvae is predominantly due to JHEH associated with the membranous fractions of cells, and JH hydrolysis in prepupae is due predominantly to soluble JHE. However, in final instar larvae of $D$. hydei, JH-acid, the product of JHE, is a major metabolite of JHI in the fat body and body wall (29\% and $39 \%$, respectively). $\mathrm{JH}$-acid-diol is also a significant product but $\mathrm{JH}$-diol was not found (Klages and Emmerich, 1979). It remains to be determined whether the apparently higher proportion of JHE activity in $D$. hydei larvae compared with $D$. melanogaster indicates poor hydrolysis of the non-physiological JHI by JHEH, localisation of JHE to the two tissues tested, or a real difference between these two species. In Lepidoptera, both JHE in haemolymph and JHE and JHEH in other tissues are important at both prewandering and prepupal stages, although evidence from three lepidopterans suggests a trend for a higher proportion of JHEH at the prewandering stage compared with the prepupal stage (Section 1.6.1). Therefore, the relative activities of the two hydrolytic enzymes at each stage seems variable, but a notable contrast is the prominence of haemolymph JHE in prewandering lepidopterans and its absence in prewandering dipterans.

Although the available evidence suggests that the temporal patterns of $\mathrm{JH}$ hydrolysis are similar among dipterans, the reported activities are very different between three Drosophila species. The highest JHE activities reported for $D$. hydei and $D$. virilis prepupae/pupae are less than in D. melanogaster by approximately 2-3 or 5 orders of magnitude, respectively (Klages and Emmerich, 1979; Rauschenbach et al., 1991). The maximum activity of $D$. hydei JHE was certainly underestimated because a non-physiological isoform, JHI, was used 180 -fold lower than its $\mathrm{K}_{\mathrm{M}}$ concentration 
(Bisser and Emmerich, 1981) whereas a saturating concentration of JHIII was used with $D$. melanogaster JHE. For $D$. virilis, comparison between the reported enzyme activities and the assay method suggests that a calculation error may have caused activities to be understated by a factor of at least 1000. In contrast, the results of this study indicate that JHE activities in $D$. virilis pupal homogenates are about a tenth of the activity found in D. melanogaster. Thus, it is possible that the levels of JH hydrolytic activities are similar between these three Drosophila species.

\subsubsection{Role of JH Hydrolysis in Regulation of JH Titre}

Comparison between the JHIII titres of D. melanogaster (Bownes and Rembold, 1987; Sliter et al., 1987) and peak levels of JH hydrolysis suggests that the hydrolytic capacity is sufficient to entirely remove $\mathrm{JH}$ within several milliseconds. Even the minimum levels of hydrolysis represent a high capacity, although the in vivo titres of $\mathrm{JHB}_{3}$ are not known and may exceed the JHIII titre (Richard et al., 1989b). Two explanations for a high JH hydrolytic capacity are proposed. Firstly, virtually all JH may be bound to lipophorin in final instar D. melanogaster (Shemshedini and Wilson, 1988), suggesting that a very high capacity for hydrolysis may be required for mass action to favour $\mathrm{JH}$ hydrolysis over $\mathrm{JH}$ binding to lipophorin. Alternatively, a high rate of hydrolysis may be matched by a high rate of synthesis, as proposed for larvae of $T$. $n i$, to enable large and rapid changes of $\mathrm{JH}$ titre to occur in response to relatively small changes in the rates of synthesis or hydrolysis (Hanzlik and Hammock, 1988). However, the second proposal seems less likely because maximum in vitro rates of $\mathrm{JH}$ production by $D$. melanogaster ring glands are less than $0.05 \mathrm{pmol} / \mathrm{hour} / \mathrm{gland}$ (Richard et al., 1990), a much lower rate of synthesis than the hydrolytic capacity.

The parallels between the expression pattern of $\mathrm{JH}$ hydrolysing enzymes in D. melanogaster, other dipterans and the lepidopterans suggest that $\mathrm{JH}$ hydrolysis plays an important role in the regulation of JH titre, as proposed for other insects (Section 1.5). Furthermore, JHIII hydrolytic capacity through development is negatively correlated with the profile of JHIII titre (Bownes and Rembold, 1987; Sliter et al., 1987; Fig. 2.8). Although there are no data on the developmental profile of $\mathrm{JHB}_{3}$ titre, hydrolysis is also negatively correlated with the profiles of in vitro JHIII and $\mathrm{JHB}_{3}$ production (Richard et al., 1989a; Altaratz et al., 1991; Dai and Gilbert, 1991; Fig. 2.8). The prewandering peak of JHIII hydrolytic activity in D. melanogaster coincides with a low JH titre. Conversely, the low levels of JH hydrolysis in post-wandering larvae and at the white prepuparium stage coincide with maximum $\mathrm{JH}$ production and an elevated JH titre. One (or several) hour(s) after the formation of the puparium the production of $\mathrm{JH}$ by the ring gland declines rapidly. Total $\mathrm{JH}$ hydrolysis changes about 26 -fold from its minimum to its second and larger maximum value within 6 hours of the prepupae becoming bouyant (between 6 and 12 hours after the formation of the puparium). Thus, the greatest and most rapid change in hydrolysis of $\mathrm{JH}$ immediately preceeds the pupal moult (Bainbridge and Bownes, 1981) towards the end of a brief period (from immediately prior to the formation of the puparium up to about 12 to 15 hours later) identified as critical for the development of adult abdominal morphology, and during which $D$. melanogaster is most sensitive to topical application of $\mathrm{JH}$ or $\mathrm{JH}$ analogues (Bhaskaran, 1972; Postlethwait, 1974). JH hydrolytic activity remains high though declining and $\mathrm{JH}$ titre is low during most of the pupal stage. In adults an elevated $\mathrm{JH}$ titre and $\mathrm{JH}$ synthesis coincide with low hydrolytic activity. 
Figure 2.8 Comparison of the Ecdysteroid Titre, JH Titre, JH Hydrolysis and JH Production During the Development of D. melanogaster

This figure combines the data presented in figure 1.2 (A, B and D) and figure 2.6 (C). Pupal age is indicated from pupariation although the larva/pupa transition occurs at pupation (pupal apolysis), twelve hours later (Section 1.4.1).

(A) shows the titre of ecdysteroids during development and is reproduced from Riddiford (in press). The data were compiled from ten sources cited therein.

(B) shows the titre of JHIII during development and is reproduced from Riddiford (in press). Solid bars indicates male titres and open bars indicate female titres. The data were compiled from Bownes and Rembold (1987) and Sliter et al. (1987).

(C) shows the activities of JHE (triangles) and JHEH (circles) during development. The developmental time axis has been altered slightly from figure 2.6 to align with the other data.

(D) shows in vitro $\mathrm{JH}$ production by brain/ring gland/ventral ganglion complexes or adult corpora allata. Solid symbols indicate $\mathrm{JHB}_{3}$ production and open symbols indicate JHIII production. The data were compiled from Richard et al. (1989a), Altaratz et al. (1991) and Dai and Gilbert (1991). 


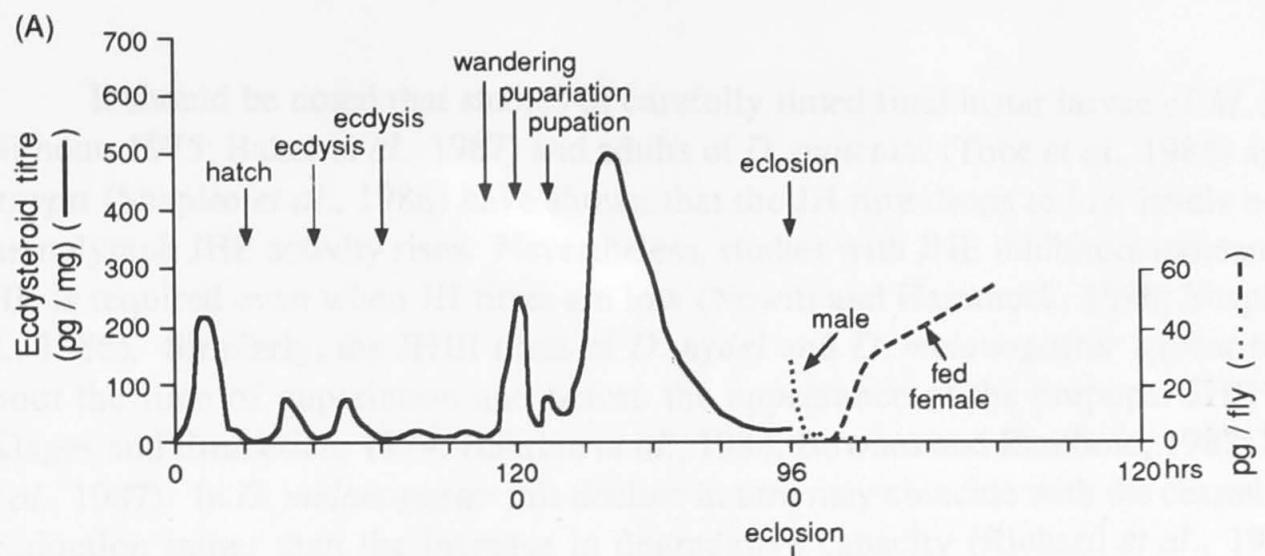

(B)

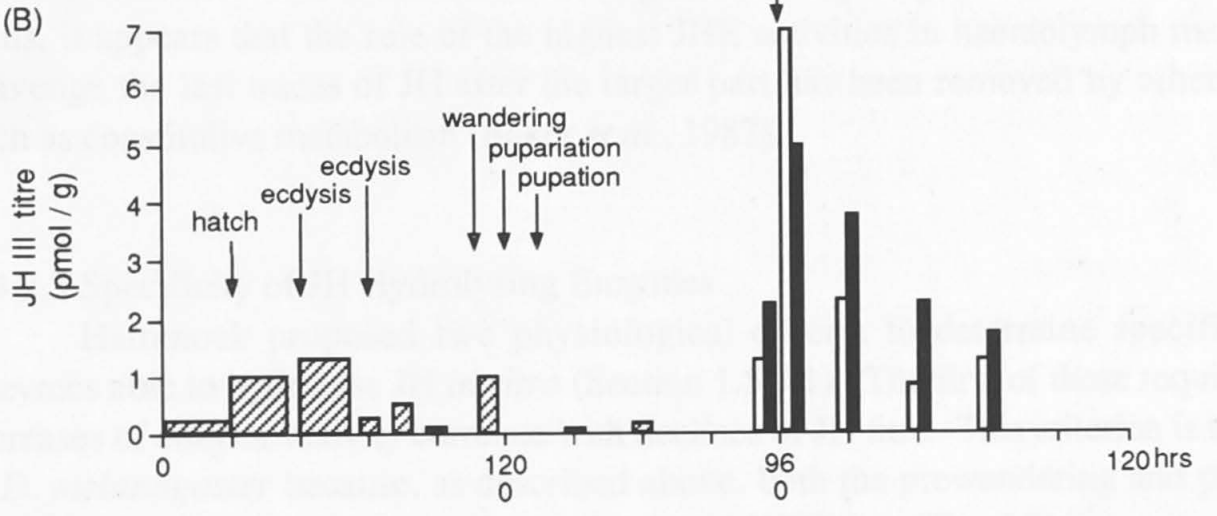

(C) 1000 pupation
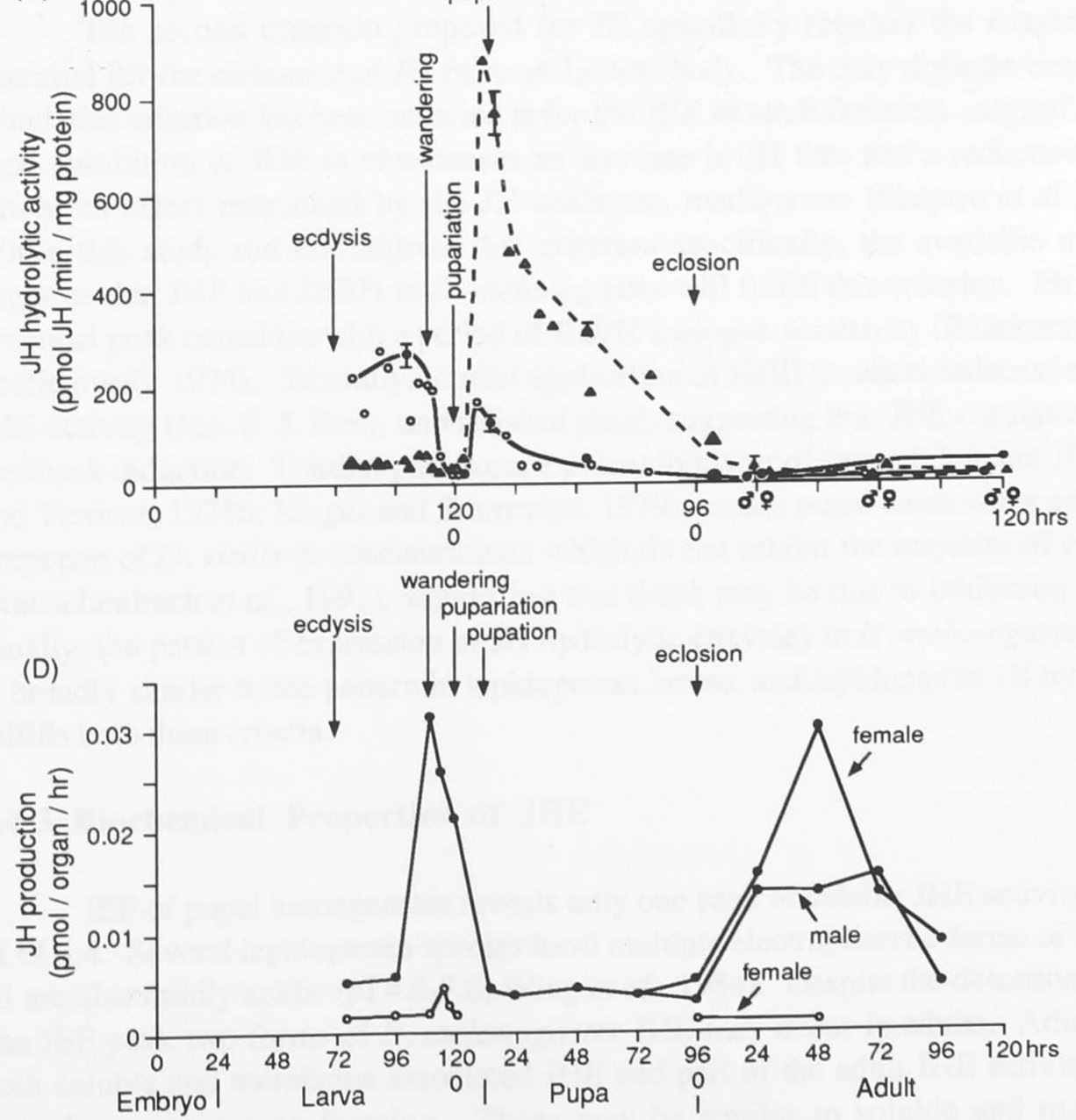
It should be noted that studies of carefully timed final instar larvae of $M$. sexta (Nijhout, 1975; Baker et al., 1987) and adults of D. punctata (Tobe et al., 1985) and A. aegypti (Shapiro et al., 1986) have shown that the JH titre drops to low levels before haemolymph JHE activity rises. Nevertheless, studies with JHE inhibitors indicate that JHE is required even when JH titres are low (Newitt and Hammock, 1986; Shapiro et al., 1986). Similarly, the JHIII titres of $D$. hydei and D. melanogaster appear to fall about the time of pupariation and before the appearance of the prepupal JHE peak (Klages and Emmerich, 1979; Bührlen et al., 1984; Bownes and Rembold, 1987; Sliter et al., 1987). In D. melanogaster this decline in titre may coincide with the cessation of production rather than the increase in degradative capacity (Richard et al., 1989a). Thus, it appears that the role of the highest JHE activities in haemolymph may be to scavenge the last traces of $\mathrm{JH}$ after the larger part has been removed by other means such as constitutive metabolism (Baker et al., 1987).

\subsubsection{Specificity of JH Hydrolysing Enzymes}

Hammock proposed two physiological criteria to determine specificity of enzymes able to hydrolyse JH in vitro (Section 1.5.3.1). The first of these requires that increases of enzyme activity correlate with declines of $\mathrm{JH}$ titre. This criterion is satisfied in D. melanogaster because, as described above, both the prewandering and prepupal peaks of JH hydrolysis occur at times of reduced JHII titre (Fig. 2.8).

The second criterion proposed for $\mathrm{JH}$ specificity requires the enzyme to be essential for the clearance of JH from an insects body. The only dipteran example in which this criterion has been satisfied is for the JHE of adult female A. aegypti. In this case, inhibition of JHE in vivo causes an increase in JH titre and a reduction in egg hatch, an effect mimicked by the JH analogue, methoprene (Shapiro et al., 1986). While this study did not address this criterion specifically, the available evidence suggests that JHE and JHEH in D. melanogaster will fulfill this criterion. Firstly, the prepupal peak coincides with a period of $\mathrm{JH} / \mathrm{JH}$ analogue sensitivity (Bhaskaran, 1972; Postlethwait, 1974). Secondly, topical application of JHIII to adults induces increased JHE activity (Ms. S. J. Dent, unpublished data), suggesting that JHE regulates JH via feedback induction. Thirdly, paraoxon, a potent inhibitor of several dipteran JHEs (Yu and Terriere, 1978b; Klages and Emmerich, 1979), causes pupal death when applied to prepupae of $D$. virilis at concentrations which do not inhibit the majority of esterases (Rauschenbach et al., 1991), suggesting that death may be due to inhibition of JHE. Finally, the pattern of expression of $\mathrm{JH}$ hydrolytic enzymes in D. melanogaster larvae is broadly similar to the pattern in lepidopteran larvae, and lepidopteran JH hydrolysis fulfills both these criteria.

\subsubsection{Biochemical Properties of JHE}

IEF of pupal homogenates reveals only one peak of soluble JHE activity, with a pI of 5.4. Several lepidopteran species have multiple electrophoretic forms of JHE but all are also mildly acidic (pI 4.0-7.0; Wing et al., 1984). Despite the detection of only one IEF peak two forms of $D$. melanogaster JHE may occur in adults. Adults have both soluble and membrane associated JHE and part of the adult JHE activity is lost from homogenates on freezing. These may be similar to soluble and membrane 
associated forms of JHE in the dipteran, $M$. domestica, which exhibit different stability and inhibitor sensitivities (Sparks and Hammock, 1980).

Previous inhibitor studies have suggested that. JHEs in Diptera may differ structurally from those of other insect orders (Section 1.7.1). In this study, JHEs from $D$. melanogaster and $D$. virilis were sensitive to both of the inhibitors tested. One compound, OTFP, is an esterase transition state analogue that shows high specificity for the JHEs of Lepidoptera (Hammock et al., 1984; Abdel-Aal and Hammock, 1986). OTFP inhibited the JHEs of both Drosophila species at $10^{-5} \mathrm{M}$, although several other esterases of $D$. melanogaster are also inhibited by this concentration (Healy et al., 1991). The other compound, DFP is an organophosphate and both compounds act by binding to the active site serine of carboxylesterases. It has been suggested that resistance to inhibition by DFP is a universal characteristic of JHEs (Hammock, 1985). However, the JHEs of the dipterans in this study, as well as $S$. bullata and $P$. regina (Yu and Terriere, 1978a), but not M. domestica (Sparks and Hammock, 1980), are sensitive to inhibition by DFP. Since several of the soluble, naphthylacetate hydrolysing esterases of Drosophila are also resistant to DFP (Healy et al., 1991), it would appear that DFP resistance is neither a unique nor universal characteristic of JHEs. 


\section{Chapter 3 \\ Biochemical Characterisation of Purified JHE}

\subsection{Introduction.}

My aim in this section of the work was to characterise D. melanogaster JHE biochemically to further assess its role in $\mathrm{JH}$ metabolism. To achieve this overall aim, four specific issues were addressed.

Firstly, a purification protocol for JHE from D. melanogaster was developed as the use of purified JHE would eliminate possible interference from other molecules during kinetic characterisation. JHE has been purified from seven lepidopteran species (Section 1.7.2) but only in $T$. $n i$ has it been purified from starting material other than haemolymph. Moreover, it has not been purified from any species outside the Lepidoptera. Two approaches to the purification of $D$. melanogaster JHE were used in this study. The first was an affinity chromatography technique based on the binding of an esterase transition state analogue, an approach which has been used to purify JHE from five lepidopterans. Conventional chromatographic techniques were used in the second approach and JHEs from four lepidopterans have been purified using such techniques. Both approaches have yielded sufficient active lepidopteran JHE for further characterisation (Section 1.7.2).

Secondly, the purified esterase was characterised kinetically to test its JHspecificity against Hammock's (1985) biochemical criteria (Section 1.5.3.1). These criteria relate directly to the action of JHE in vivo, addressing the issues of whether $\mathrm{JH}$ is likely to be the predominant in vivo substrate and whether the binding affinity and catalytic efficiency of JHE are sufficiently high to remove $\mathrm{JH}$ at the low in vivo concentrations. Kinetic analysis of lepidopteran JHEs revealed that they exhibit low $\mathrm{K}_{\mathrm{MS}}$ and high specificity constants $\left(\mathrm{K}_{\mathrm{cat}} / \mathrm{K}_{\mathrm{M}}\right)$ for JH (Section 1.7.3). These values indicate that the enzyme is well adapted to efficient hydrolysis of $\mathrm{JH}$ at low concentrations, possibly in competition with other high affinity JH binding proteins.

Thirdly, the features of the JH molecule which promote binding by JHE were determined. In particular, the unresolved issue of the relative importance of JHIII versus $\mathrm{JHB}_{3}$ was addressed by binding studies intended to determine whether JHE exhibited specificity for one of these isoforms over the other. Recent studies have investigated $\mathrm{JH}$ synthesis, $\mathrm{JH}$ metabolism by $\mathrm{JHEH}$, and some in vivo effects of these two JHs (Section 1.2). Taken together these studies indicate an important role for $\mathrm{JHB}_{3}$. However they are equivocal with regard to the role of JHIII.

The final aim was to determine the mode of interaction between JHE and lipophorin, the haemolymph JH carrier. Two distinct models for this interaction (Section 1.5.2) were tested using kinetic experiments. The first is a mass action model which depends only on the relative abundance and kinetic properties of the carrier and degrading enzymes to determine the rate of JH degradation. The second model requires direct contact between JHE and the carrier and has only been demonstrated in one species in which the carrier is also a non-competitive inhibitor of JHE. 


\subsection{Materials and Methods}

\subsubsection{Chemicals}

The $\mathrm{JH}$ isoforms and analogues not previously described in Chapter 2 or referenced otherwise were synthesised in the laboratory of Professor R. W. Rickards of The Australian National University (see Rickards and Thomas, 1992 for synthesis of four stereoisomers of $\mathrm{JHB}_{3}$ ). OTFP was synthesised by Dr. C. P. Whittle (methods of Abdel-Aal and Hammock, 1986), L. cuprina lipophorin was purified by density gradient centrifugation by Dr. S. C. Trowell (Trowell, 1992) and D. melanogaster EST6 was purified by Ms. M. M. Dumancic (Division of Entomology, C.S.I.R.O.). Commercially produced compounds were S-methoprene, hydroprene (provided by Zoecon), JHI, JHII and JHIII (Sigma).

\subsubsection{JHE Assay, Electrophoretic, and Protein Detection Procedures}

\subsubsection{JHE Activity Assay}

The JHE partition assay described in section 2.2.2.6 was modified for the monitoring of JHE activity through its purification and for the kinetic studies of JHE. For the purification the assay volume was reduced to $100 \mu \mathrm{l}$, BBA was not used and $0.5 \mathrm{mg} / \mathrm{ml} \mathrm{BSA} \mathrm{(Sigma)} \mathrm{was} \mathrm{included.} \mathrm{The} \mathrm{addition} \mathrm{of} \mathrm{BSA} \mathrm{was} \mathrm{necessary} \mathrm{to} \mathrm{retain}$ enzyme activity when the enzyme preparation contained little other protein. For the kinetic experiments some further changes were made. The ${ }^{3} \mathrm{H}-\mathrm{JHIII}$ concentration was varied, potential inhibitors were included in the assay, as described in section 3.2.4, and the assay tubes were pretreated with $2 \%$ polyethyleneglycol ("Carbowax" Compound 4000 , Union Carbide) to reduce adsorption of JH (and its analogues) to the glass (Giese et al., 1976). This technique has been shown to reduce the proportion of very dilute JHIII bound to glassware from $78 \%$ to $44 \%$, although surface binding of $\mathrm{JH}$ probably remains the main source of error in assays performed with less-than-saturating $\mathrm{JH}$ concentrations (Lanzrein et al., 1993).

\subsubsection{Electrophoretic Procedures}

\section{Native PAGE}

Staining for naphthylacetate hydrolysis and extraction of JHE activity from native PAGE were performed as described in sections 2.2.2.2 and 2.2.2.4. Electrophoresis was performed at a constant $5 \mathrm{~W}$ per gel $(70 \times 80 \times 0.75 \mathrm{~mm})$ with circulating iced water for cooling.

\section{Denaturing PAGE}

Denaturing (SDS) PAGE was performed by the method of Laemmli (1970) using 12\% 40:1 acrylamide/bis-acrylamide. Electrophoresis was performed at room temperature with circulating water for cooling at a constant $150-200 \mathrm{~V}$. Gels were $70 \mathrm{x}$ $80 \times 0.75$ or $1 \mathrm{~mm}$.

\section{Two Dimensional PAGE}

Samples were firstly fractionated by native PAGE. Replicate lanes of the native PAGE gel were stained for naphthylacetate hydrolysis, or total protein, or sliced and assayed for JHE activity. A gel piece corresponding to the region with JHE activity was 
excised from an unstained lane, incubated with denaturing gel sample buffer (Laemmli, 1970) for approximately 15 minutes, then subjected to SDS-PAGE as the second dimension.

All polyacrylamide gels described in this chapter were air-dried between cellophane using a Promega Gel Drying Kit.

\subsubsection{Protein Detection and Sequencing Procedures}

After fractionation by PAGE, proteins were detected by the silver staining method of Merril et al. (1980) using the Biorad silver staining kit. The protein content of solutions was determined by the method of Bradford (1976) using the Biorad Protein Assay kit, with BSA as the standard.

N-terminal amino acid sequencing of purified JHE (20 $\mu$ g of Fr6, Section 3.3.1) was performed by Edman degradation by the Biomolecular Resource Unit at the John Curtin School of Medical Reseach, Australian National University. The EMBL database (Release 27.0) was searched for sequences with similarity to the N-terminal amino acid sequence of JHE (Devereux et al., 1984).

\subsubsection{Purification of JHE}

Two approaches were taken to the purification of JHE, an affinity chromatography procedure and a conventional, chromatographic approach. The initial steps (homogenisation, ammonium sulphate and acetone precipitations) were common to both approaches.

\subsubsection{Initial Purification Steps}

\section{Preparation of Homogenates}

The D. melanogaster strain 12I11.2 (Cooke et al., 1987) was cultured in population cages under the conditions described in section 2.2.1.1 except that the food was regularly moistened to ensure that most larvae pupated on the walls of the cage. Wandering larvae and pupae were removed from the walls of the cage by washing with a strong jet of water. Pupae were aged by the flotation method of Mitchell and Mitchell (1964) to $12 \pm 6$ hours (the time of maximum JHE activity, Section 2.3.2.2) and then stored at $-70^{\circ} \mathrm{C}$.

$100 \mathrm{~g}$ of frozen pupae were homogenised on ice in a Sorvall blender with a few milligrams of PTU and $500 \mathrm{ml}$ of a $\mathrm{pH} 4.5$ buffer $(10 \mathrm{mM} \mathrm{KOH}$ adjusted with acetic acid). The homogenate was clarified by centrifugation at $13,000 \mathrm{~g}, 4^{\circ} \mathrm{C}$ for 20 minutes followed by filtration through glass wool. The $\mathrm{pH}$ of this solution was then raised by the addition of an equal volume of $100 \mathrm{mM}$ sodium phosphate buffer, $\mathrm{pH} 7.0$ containing $10 \%$ glycerol.

\section{Ammonium Sulphate Precipitation}

Solid ammonium sulphate was added slowly to the homogenate with stirring at $4^{\circ} \mathrm{C}$ until $30 \%$ saturation was reached. The solution was stirred gently for a further 60 minutes and then centrifuged for 20 minutes at $13000 \mathrm{~g}$ at $4^{\circ} \mathrm{C}$. The supernatant was brought to $60 \%$ saturation by slow addition of solid ammonium sulphate, stirred for a further 60 minutes and then the precipitate was pelleted as above. The supernatant was 
discarded and the pellet was resuspended in $100 \mathrm{mM}$ sodium phosphate buffer, $\mathrm{pH} 7.0$ containing $10 \%$ glycerol to a final volume of $52 \mathrm{ml}$.

\section{Acetone Precipitation}

Acetone $\left(0^{\circ} \mathrm{C}\right)$ was slowly added to the resuspended ammonium sulphate pellet with vigorous swirling in an ice/water slurry until a final concentration of $40 \%(\mathrm{v} / \mathrm{v})$ was reached. The resulting solution was centrifuged at $9000 \mathrm{~g}$ for 15 minutes at $0^{\circ} \mathrm{C}$ in $30 \mathrm{ml}$ Corex tubes. The supernatant was dialysed overnight at $4^{\circ} \mathrm{C}$ against 31 of 10 $\mathrm{mM}$ imidazole/ $\mathrm{Cl}$ buffer, $\mathrm{pH} 7.0$ containing $10 \%$ glycerol. This material was concentrated to less than $50 \mathrm{ml}$ using aquacide III (Calbiochem 17852).

\subsubsection{Affinity Purification of JHE}

Two versions of trifluoromethylketone affinity matrix were made (Fig. 3.1). Briefly, 1,4 butanedithiol was reacted with 3 bromo 1,1,1 trifluoropropanone, to produce 3-[(4'-mercapto)butylthio]-1,1,1-trifluoropropan-2-one, which was then coupled to either Thiopropyl Sepharose 6B (Pharmacia, Hanzlik, 1988) or Epoxyactivated Sepharose 6B (Pharmacia, Abdel-Aal and Hammock, 1986) following the manufacturers instructions. The former incorporates a disulphide bond into the linker between the sepharose bead and the trifluoromethylketone moiety. Reduction of the disulphide bond enables greater recovery of bound protein but recovery of enzyme activity is not expected by this method (Hanzlik, 1988).

The procedures used for affinity purification of the lepidopteran JHEs (Abdel-Aal and Hammock, 1986; Hanzlik and Hammock, 1987) were followed, except that the starting material was not pre-treated with DFP (which inhibits D. melanogaster JHE, Section 2.3.2.4). The prepared matrix was washed with copious quantities of ethanol and water (about $50 \mathrm{ml}$ ) in varying proportions to remove any unbound ligand before equilibration with $100 \mathrm{mM}$ sodium phosphate buffer, $\mathrm{pH} 7.0$.

Partially purified JHE (Section 3.2.3.1) was incubated with either affinity matrix type $(100-1000 \mu \mathrm{l})$ for several hours at room temperature or overnight at $4^{\circ} \mathrm{C}$, with rocking. The matrix was washed at room temperature with copious volumes of phosphate $(50-1000 \mathrm{ml})$ buffer that contained $0.1 \%$ Triton $\mathrm{X}-100$, then treated to remove bound proteins. The disulphide linked matrix was cleaved by incubation with $20 \mathrm{mM}$ dithiothrietol or $B$-mercaptoethanol. Proteins that bound to the non-disulphide linked matrix were eluted by incubation of the matrix with 1-2 ml of $10^{-3} \mathrm{M}$ OTFP, a free form of the affinity ligand, for several hours at room temperature, then overnight at $4^{\circ} \mathrm{C}$, with rocking. The eluted proteins were concentrated by ultrafiltration in a Centriprep 30 or Centricon 30 apparatus (Amicon) and then analysed by SDS-PAGE. The concentrated OTFP eluate was then subjected to very extensive dialysis at $4{ }^{\circ} \mathrm{C}$ (up to one week with daily changes of 21 of $200 \mathrm{mM}$ sodium phosphate buffer, $\mathrm{pH} 7.4$, containing $10 \%$ glycerol, $0.02 \% \mathrm{NaN}_{3}, 0.01 \%$ PTU, and $1 \mathrm{mM} \mathrm{B-mercaptoethanol)} \mathrm{to}$ remove the OTFP from the eluted protein and thereby recover enzyme activity.

\subsubsection{Conventional Purification}

\section{Isoelectric Focussing}

Isoelectric Focussing (IEF) was performed using a Biorad "Rotofor" apparatus. Three $\mathrm{ml}$ of ampholytes (Pharmalyte $\mathrm{pH} 5-6$, Pharmacia) and 10\% glycerol were added to partially purified JHE (Section 3.2.3.1), bringing the total volume to $50 \mathrm{ml}$, the volume of the Rotofor focussing chamber. In accordance with the manufacturer's 
A<smiles>O=C(CSCCCCS)C(F)(F)F</smiles>

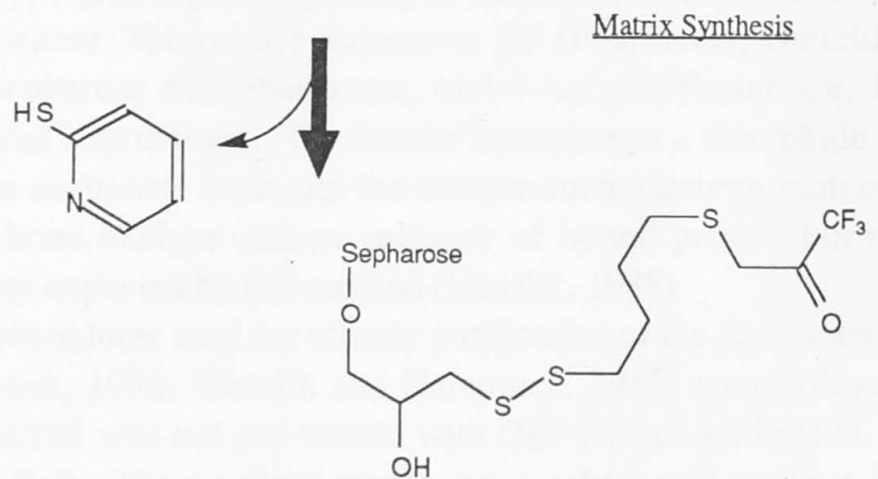

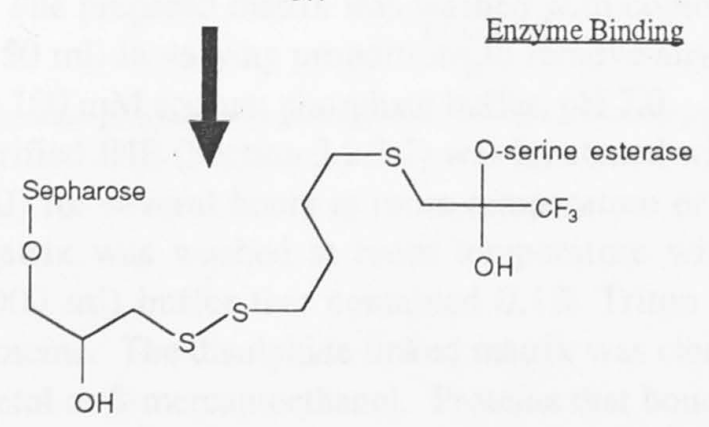<smiles>[R3]CCC</smiles>

Enzyme Elution<smiles>OC(CS)CO[GeH3]</smiles> 


\section{B}
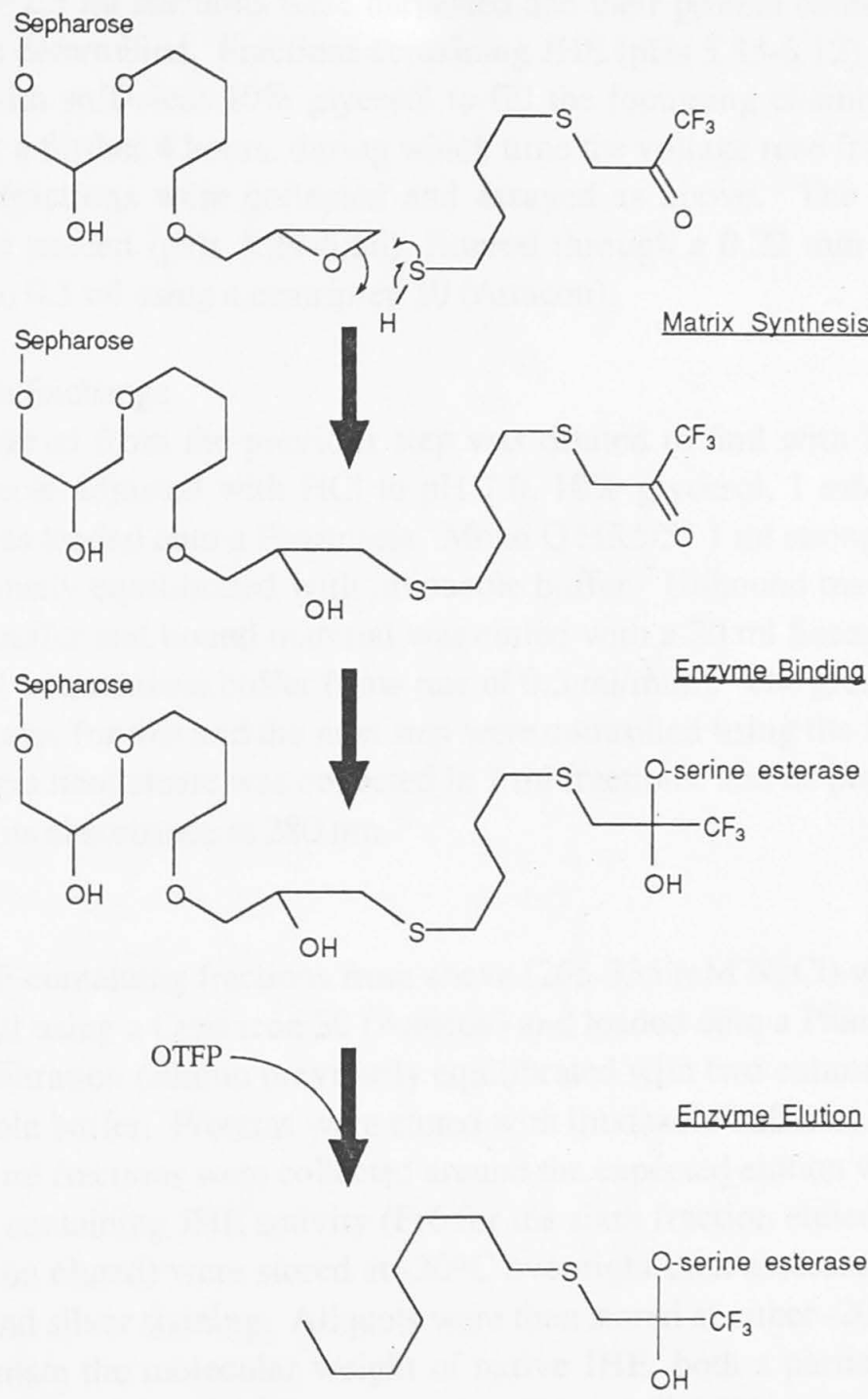

Figure 3.1 Synthesis and Use of Matrices for Trifluoromethylketone Affinity Chromatography

3-[(4'-mercapto)butylthio]-1,1,1-trifluoropropan-2-one was coupled to either Thiopropyl Sepharose 6B (A, Hanzlik, 1988) or Epoxy-activated Sepharose 6B (B, Abdel-Aal and Hammock, 1986). Esterases bind to the trifluoromethylketone group covalently through their active site serine. In (A), a disulphide bond is incorporated into the linker between the sepharose bead and the trifluoromethylketone group. Esterases are recovered by reduction of the disulphide bond. In (B), esterases are recovered from the non-disulphide linked version by slow exchange for OTFP which also contains the trifluoromethylketone moiety. Extensive dialysis is then required to remove OTFP from the active site and recover enzyme activity. 
instructions an antifreeze solution at $-8^{\circ} \mathrm{C}$ was circulated through the apparatus to achieve a temperature in the focussing chamber of around $2^{\circ} \mathrm{C}$. The sample was focussed for 4 hours at 12 watts, during which time the voltage rose from 300 to 880 volts. Twenty $2.5 \mathrm{ml}$ fractions were harvested and their protein content, $\mathrm{pH}$ and JHE activities were determined. Fractions containing JHE ( $\mathrm{pHs} 5.35-6.12$ ) were returned to the Rotofor with sufficient $10 \%$ glycerol to fill the focussing chamber. These were refocussed for a further 4 hours, during which time the voltage rose from 1600 to 1900 volts. Then fractions were collected and assayed as above. The JHE containing fractions were pooled ( $\mathrm{pHs}$ 5.29-5.56), filtered through a $0.22 \mathrm{~mm}$ membrane, and concentrated to $0.5 \mathrm{ml}$ using a centriprep 30 (Amicon).

\section{Mono Q Anion Exchange}

The material from the previous step was diluted to $5 \mathrm{ml}$ with imidazole buffer (0.1 M imidazole adjusted with $\mathrm{HCl}$ to $\mathrm{pH} 7.0,10 \%$ glycerol, $1 \mathrm{mM}$ dithiothrietol). The sample was loaded onto a Pharmacia "Mono Q HR5/5" $1 \mathrm{ml}$ strong anion exchange column previously equilibrated with imidazole buffer. Unbound material was eluted with $6 \mathrm{ml}$ of buffer and bound material was eluted with a $20 \mathrm{ml}$ linear gradient of 0 to $500 \mathrm{mM} \mathrm{NaCl}$ in imidazole buffer (flow rate of $0.5 \mathrm{ml} / \mathrm{min}$.). The gradient for this step and the flow rates for this and the next step were controlled using the Pharmacia FPLC system. The gradient eluate was collected in $1 \mathrm{ml}$ fractions, and its protein content was monitored by its absorbance at $280 \mathrm{~nm}$.

\section{Gel Filtration}

The JHE containing fractions from above $(265-335 \mathrm{mM} \mathrm{NaCl})$ were concentrated to about $100 \mu \mathrm{l}$ using a Centricon 30 (Amicon) and loaded onto a Pharmacia "Superose 6" $30 \mathrm{ml}$ gel filtration column previously equilibrated with two column volumes of the above imidazole buffer. Proteins were eluted with imidazole buffer at a flow rate of 0.5 $\mathrm{ml} / \mathrm{min}$ and $1 \mathrm{ml}$ fractions were collected around the expected elution volume $(16.5 \mathrm{ml})$. The fractions containing JHE activity ( $\mathrm{Fr} 6$ for the sixth fraction eluted, and $\mathrm{Fr} 7$ for the seventh fraction eluted) were stored at $-20^{\circ} \mathrm{C}$ overnight then assessed for purity using SDS-PAGE and silver staining. Aliquots were then stored at either $-20^{\circ} \mathrm{C}$ or $-70^{\circ} \mathrm{C}$.

To estimate the molecular weight of native JHE, both a partially purified JHE preparation and a mixture of known proteins (Biorad Gel Filtration Standards) were separated on a $320 \mathrm{ml}$ Pharmacia "Superdex 200" gel filtration column using imidazole buffer and a flow rate of $1.0 \mathrm{ml} / \mathrm{min}$.

\subsubsection{Kinetic Characterisation of JHE}

Kinetic experiments were all performed using material conventionally purified to near homogeneity (Fr7, characterised in sections 3.3.1.2 and 3.3.2.1).

\subsubsection{Kinetic Analysis of JHIII Hydrolysis and Inhibition by JH Isoforms}

The type of inhibition and the inhibition constants were determined for three isoforms of $\mathrm{JH}$ ( $\mathrm{JHI}, \mathrm{JHB}_{3}$, and MF). In each experiment, assays (Section 3.2.2.1) were performed with various concentrations of both the JHIII and inhibitor. A series of JHIII concentrations $(0.0555,0.0715,0.1,0.1665,0.5 \mu \mathrm{M}, 3871 \mathrm{cpm} / \mathrm{pmol})$ were chosen to yield equally spaced data points on a Lineweaver-Burke (double reciprocal) plot and span the $\mathrm{K}_{\mathrm{M}}$ concentration of JHIII. ${ }^{3} \mathrm{H}-\mathrm{JHIII}$ was added to the assay solution 
in $1 \mu \mathrm{l}$ ethanol whereas the $\mathrm{JH}$ isoforms were added in $1 \mu \mathrm{l}$ dimethylsulphoxide (DMSO). Reactions were started by the addition of $3 \mu \mathrm{l}$ of JHE (fraction 7 diluted 1/20 in $5 \mathrm{mg} / \mathrm{ml} \mathrm{BSA}$ ). Incubation times for these experiments were 20 minutes, during which time a maximum of $20 \%$ of the substrate was consumed and the reaction rates were linear. Each inhibition experiment also provided an estimate of the Michaelis constant $\left(\mathrm{K}_{\mathrm{M}}\right)$ for hydrolysis of JHIII by JHE. For each isoform, the experiment was repeated three times, providing three data sets.

After graphical determination of the type of inhibition, an overall fit of each set of data was made to the appropriate rate equation (in this case competitive inhibition) using the appropriate computer program of Cleland (1979). An example of the computer analysis is shown in the appendix. Weighted means of the inhibition constants from each data set were then calculated. The reaction rate per mole of JHE ( $\left.\mathrm{K}_{\text {cat }}\right)$ for JHIII was calculated using the specific activity of Fr6 measured soon after purification because the aliquots of Fr7 used for these experiments had been stored for a few days at $-20^{\circ} \mathrm{C}$, resulting in the loss of $97 \%$ of the activity measured immediately after purification. However, no loss of activity occurred during subsequent storage at $-70^{\circ} \mathrm{C}$. The constants $\mathrm{K}_{\mathrm{M}}$ and $\mathrm{K}_{\mathrm{I}}$ are independent of the absolute amount of enzyme used in each experiment and so needed no correction.

\subsubsection{Kinetic Properties with $\alpha$-Naphthylacetate}

$\alpha$-Naphthylacetate was tested as an inhibitor of JHE as above (Section 3.2.4.1) and the properties of $\alpha$-naphthylacetate as a substrate for JHE were determined spectrophotometrically using the method of Mastrapaolo and Yourno (1981). Substrate at a final concentration of $25,50,100,200$ or $400 \mu \mathrm{M}$ was added in $1 \mu \mathrm{l}$ of 2 methoxyethanol to $75 \mu \mathrm{l}$ of $100 \mathrm{mM}$ sodium phosphate buffer, $\mathrm{pH} 8.0$ in a quartz microcuvette. $\alpha$-Naphthylacetate slowly hydrolyses in water so a background rate was determined before $1 \mu \mathrm{l} \mathrm{JHE}$ (Fr7 diluted $1 / 5 \mathrm{in} 10 \mathrm{mg} / \mathrm{ml} \mathrm{BSA}$ ) was added to start the enzymic reaction. This background rate was subtracted from the reaction rate with enzyme and the published extinction coefficient for $\alpha$-naphthol $\left(24.0 \mathrm{mM}^{-1} \mathrm{~cm}^{-1}\right.$ at 235 $\mathrm{nM}$ ) was used to calculate absolute rates. The temperature of the spectrophotometer chamber was a constant $33^{\circ} \mathrm{C}$ after equilibration. The small reaction volume was necessary in order to conserve JHE but evaporation from the cuvette during assays caused poor reproducibility. To minimise this effect reaction times were reduced (typically 1-2 minutes) and the spectrophotometer chamber was humidified.

Two corrections were made to the measured reaction rates of $\alpha$-naphthylacetate hydrolysis with Fr7 to account for the presence of a single, major contaminant in Fr7 which is absent from Fr6 and for loss of activity after purification. Firstly, the amount of JHE protein in Fr7 was estimated using the specific activity of the homogeneous Fr6 and the total measured activity of the two preparations. Secondly, the measured reaction rates were scaled up proportionately to reflect the original JHE activity of Fr7.

\subsubsection{Identification of JH Structural Features which Promote Binding to JHE}

In order to further define the specificity of binding by JHE, a wide range of $\mathrm{JH}$ isoforms and compounds sharing structural features with $\mathrm{JH}$ ("JHAs" for $\mathrm{JH}$ analogue; listed in Fig. 3.2) were tested as inhibitors of JHIII hydrolysis. JHE assays were performed as described in section 3.2.4.1 except that the JHA concentrations (10 $\mu \mathrm{M}$ final concentration added in $1 \mu \mathrm{l}$ DMSO $)$ and ${ }^{3} \mathrm{H}$-JHIII concentration $(0.056 \mu \mathrm{M}, 3871$ $\mathrm{cpm} / \mathrm{pmol}$ ) were fixed and reactions were started by the addition of $3 \mu \mathrm{l}$ of JHE (Fr7 


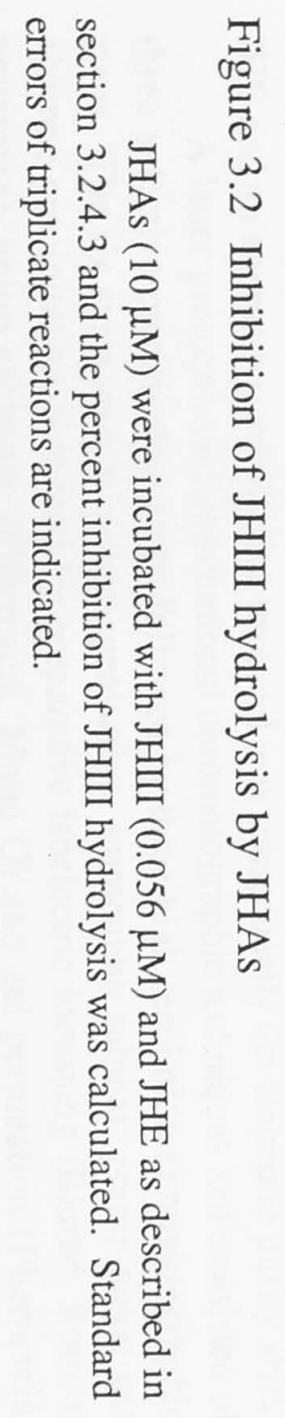

$\%$ Inhibition of JHIII hydrolysis

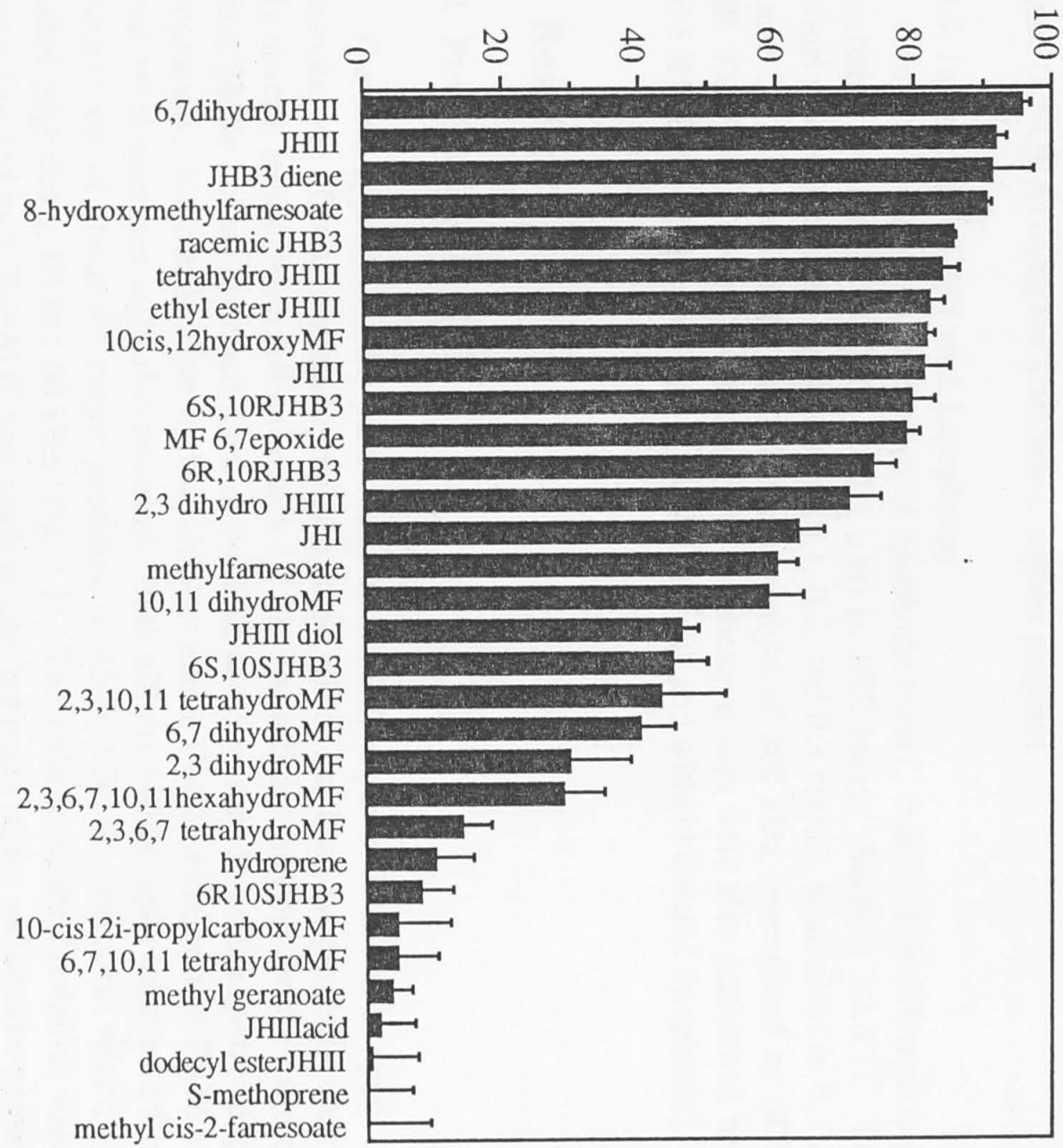


diluted $1 / 20$ in $5 \mathrm{mg} / \mathrm{ml} \mathrm{BSA}$ ). Each compound was tested in triplicate. Control assays lacked the JHA and inhibition was expressed as a percent reduction of the control activity. Where appropriate, data for JHAs sharing systematic differences were subjected to the arcsine transformation (Sokal and Rohlf, 1969) followed by analysis of variance (ANOVA) using the StatView computer program.

\subsubsection{Interaction of JHE with Lipophorin}

Combinations of concentrations of lipophorin from L. cuprina haemolymph were coincubated with JHIII $(0.0555$ to $0.5 \mu \mathrm{M})$ in JHE assays (Section 3.2.4.1). The lipophorin concentrations used were $0,0.1,0.2$, and $0.3 \mathrm{mg} / \mathrm{ml}$, equivalent to $0,0.3$, 0.6 , and $0.9 \mu \mathrm{M}$, assuming the molecular weight of $300 \mathrm{kDa}$ determined by SDSPAGE (Dr. S. C. Trowell, pers. comm.). Triplicate assays were also performed with $6.7 \mathrm{nM}$ JHIII (no lipophorin) and $5 \mu \mathrm{M}$ JHIII (with and without $0.6 \mu \mathrm{M}$ lipophorin).

\subsection{Results}

\subsubsection{Purification of JHE}

The first approach to the purification of D. melanogaster JHE was to apply the trifluoroketone affinity chromatography method which has been successfully used to purify several lepidopteran JHEs (Section 1.7.2). The affinity matrices (with or without the disulphide linker) removed most of the JHE activity from $D$. melanogaster pupal homogenates. Naphthylacetate hydrolysis after native PAGE revealed that EST6 and several other esterases were also removed. Both affinity matrix types released a fairly consistent set of about six major peptides, of $45-80 \mathrm{kDa}$ in molecular wight, and included peptides at 66 and $60 \mathrm{kDa}$ (Fig. 3.3). The yield from the disulphide linked matrix estimated by SDS-PAGE was much greater but many minor and smaller peptides were also detected. As enzymes released from the disulphide linked matrix are exposed to a strong reductant and the preparation contained many contaminants, no attempt was made to recover JHE activity. The esterase inhibitor OTFP was used to elute peptides from non-disulphide linked matrix. Extensive dialysis was used to remove OTFP from the eluate but no JHE activity was recovered after one day or one week. It was therefore not possible to readily determine which of the protein bands corresponded to $\mathrm{JHE}$ and an alternative purification protocol was subsequently developed tc purify JHE.

A latter protocol used conventional chromatographic techniques and consisted of three selective precipitation steps followed by three high resolution chromatographic steps. The six steps were acid homogenisation, ammonium sulphate precipitation and acetone precipitation, followed by preparative isoelectric focussing (Biorad, Rotofor apparatus), anion exchange (Pharmacia, Mono Q) and gel permeation (Pharmacia, Superose 6) chromatography. Table 3.1 summarises the results of the purification of JHE from whole $D$. melanogaster pupae. Overall a 429 -fold enrichment for JHE was achieved with a recovery of $0.64 \%$ (Fr6 only). However, the recovery is $1.4 \%$ if a fraction containing a single major contaminant is included (Fr6 plus Fr7), and the enrichment factor might be about double with respect to a $\mathrm{pH} 7.0$ homogenate (see below). At no point in the purification was there evidence suggesting the presence of more than one JHE isoform. 
In preliminary experiments homogenisation of pupae at $\mathrm{pH} 4.5$ and $\mathrm{pH} 7.0$ yielded similar JHE activities, but the $\mathrm{pH} 4.5$ homogenate contained about half the soluble protein. Therefore initial homogenisation was performed at this $\mathrm{pH}$. Ammonium sulphate precipitation generally achieved about two fold enrichment with about $75 \%$ yield and a large reduction in volume from about $900 \mathrm{ml}$ to $10-15 \mathrm{ml}$, which was necessary for the subsequent steps. In trials of acetone precipitation, it was found that concentrations of greater than $40 \%$ acetone were required to precipitate JHE and these concentrations also caused large losses of activity. Nevertheless precipitation with $40 \%$ acetone was included in the purification procedure because many other proteins were precipitated, leaving most of the JHE soluble and active (typically $70 \%$ yield and 4 fold enrichment). When used in the complete purification the ammonium sulphate and acetone precipitation steps gave a poorer yield and enrichment (17\%/2.2 fold) than were usually obtained in trial experiments (typically about $50 \% / 8$ fold).

JHE containing fractions from preparative IEF were refocussed to give a good yield and enrichment for this step (41\%/22 fold, Fig. 3.4). JHE focussed at a pH of 5.4 under these conditions, the same $\mathrm{pI}$ as was found for JHE after analytical IEF of crude pupal homogenates (Section 2.3.4).

JHE binds to the Mono Q anion exchange column under conditions where most of the protein remaining after the IEF step is eluted without binding (Fig. 3.5). JHE was subsequently eluted with $265-335 \mathrm{mM} \mathrm{NaCl}$, giving a yield of $79 \%$ and a 7 fold enrichment.

JHE eluted from the final gel filtration step (Superose 6, Fig. 3.6) in the 6th and 7 th fractions (Fr6, Fr7). In the complete purification this step gave only a 1.2 fold enrichment (Fr6) and a yield of 26\% (Fr6 and Fr7). However, Fr6 appeared to be homogeneous when analysed by silver stained SDS-PAGE (Fig. 3.7A) and it contained a single peptide at $66 \mathrm{kDa}$. Fr7 contained two peptides; one at a molecular weight of 66 $\mathrm{kDa}$ and another at $40 \mathrm{kDa}$. In both fractions the $66 \mathrm{kDa}$ peptide stained with similar intensity and the JHE activity was similar, suggesting that the $66 \mathrm{kDa}$ peptide is JHE. Fr6 was retained for $\mathrm{N}$-terminal aminoacid sequencing and Fr7 was retained for kinetic experiments.

\subsubsection{Properties of Purified JHE}

\subsubsection{Identification of JHE by Two-Dimensional PAGE}

Fr7 was analysed by two-dimensional PAGE to confirm the identity of the $66 \mathrm{kDa}$ peptide as JHE. Quadruplicate samples of Fr7 were first separated by native PAGE. One each of three of the replicate lanes was either cut into ten $5 \mathrm{~mm}$ pieces and assayed for JHE activity, stained with $\alpha$ - and $\beta$-naphthylacetate, or stained for total protein. Regions of equivalent $\mathrm{Rf}$ in three lanes contained JHE activity, exhibited naphthylacetate hydrolysis ( $\alpha$ preference) and contained one of the main peptide bands (Fig. 3.7B). The $\mathrm{Rf}$ of this peptide on native PAGE corresponded with that previously determined for JHE in crude homogenates (Fig. 2.4). A gel piece corresponding to this Rf from the fourth lane was then subjected to SDS-PAGE with marker proteins (Biorad) and samples of Fr6 and Fr7, and stained for total protein. The mobility of the main peptide corresponded to a molecular weight of $66 \mathrm{kDa}$, confirming that the $66 \mathrm{kDa}$ peptide in Fr6 and Fr7 is JHE (Fig. 3.7C). The identity of the $40 \mathrm{kDa}$ peptide is unknown. 


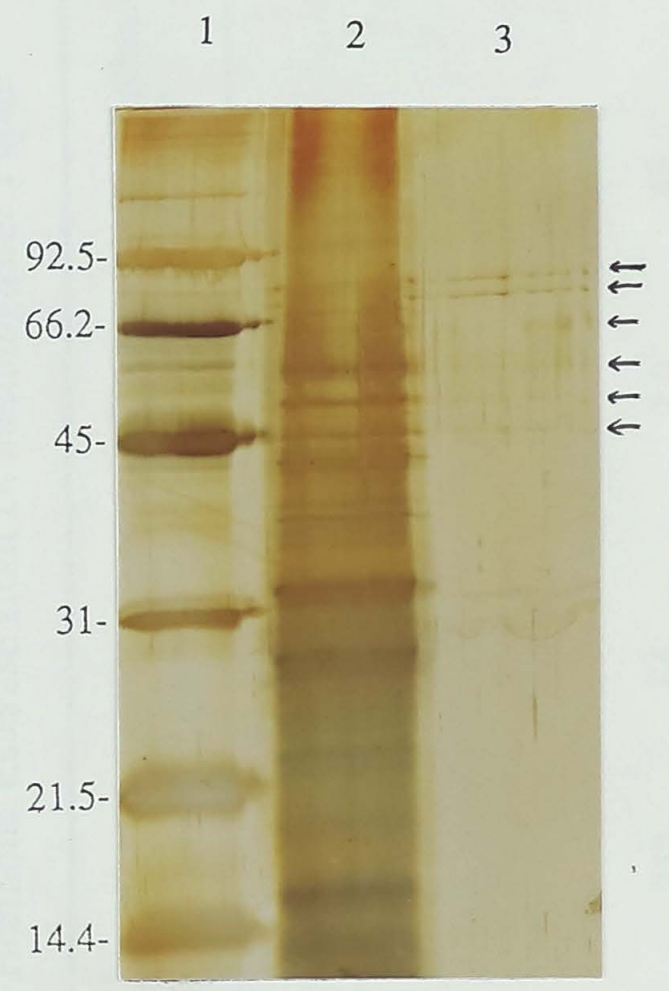

Figure 3.3 Proteins Isolated by Affinity Chromatography

Peptides from D. melanogaster pupae isolated by trifluoromethylketone affinity chromatography (Fig. 3.1), separated by denaturing PAGE and detected by silver staining as described in sections 3.2.2 and 3.2.3. Lane 1, molecular weight marker proteins with their weights $(\mathrm{kDa})$ indicated; lane 2, peptides isolated using the disulphide linked matrix; Lane 3, peptides isolated using the non-disulphide linked matrix. Peptides common to lanes 2 and 3 are indicated. 
Table 3.1. Purification of Juvenile Hormone Esterase from D. melanogaster Pupae

\begin{tabular}{lllllll}
\hline & $\begin{array}{l}\text { Volume } \\
(\mathrm{ml})\end{array}$ & $\begin{array}{l}\text { Protein } \\
(\mathrm{mg})\end{array}$ & $\begin{array}{l}\text { Total } \\
\text { activity } \\
\text { (nmol/min) }\end{array}$ & $\begin{array}{l}\text { Specific } \\
\text { Activity } \\
\text { (nmol/min/mg) }\end{array}$ & $\begin{array}{l}\text { Recovery } \\
(\%)\end{array}$ & $\begin{array}{l}\text { Purification } \\
\text { factor }\end{array}$ \\
\hline $\begin{array}{l}\text { Homogenate } \\
\text { pH4.5 } \\
\left(\mathrm{NH}_{4}\right)_{2} \mathrm{SO}_{4}\end{array}$ & 900 & 2610 & 3600 & 1.4 & - & - \\
$\begin{array}{l}\text { precipitate } \\
\text { 40\% acetone } \\
\text { supernatant }\end{array}$ & 52 & 1820 & 2880 & 1.5 & 80 & 1.1 \\
$\begin{array}{l}\text { IEF 1 } \\
\text { Rotofor }\end{array}$ & 17.5 & 36 & 344 & 9.5 & 17 & 2.2 \\
$\begin{array}{l}\text { IEF 2 } \\
\text { Rotofor }\end{array}$ & 27 & 3.85 & 252 & 66 & 10 & 6.9 \\
$\begin{array}{l}\text { Anion exchange } \\
\text { Mono Q }\end{array}$ & 3 & 0.42 & 200 & 480 & 7.0 & 48 \\
$\begin{array}{l}\text { Gel filtration } \\
\text { Superose 6 -Fr6 (7) }\end{array}$ & $1(1)$ & 0.039 & $23(28)$ & 590 & $0.64(0.78)$ & 429 \\
\hline
\end{tabular}

NB. Bracketed figures refer to a fraction containing a major contaminant. 
(A)

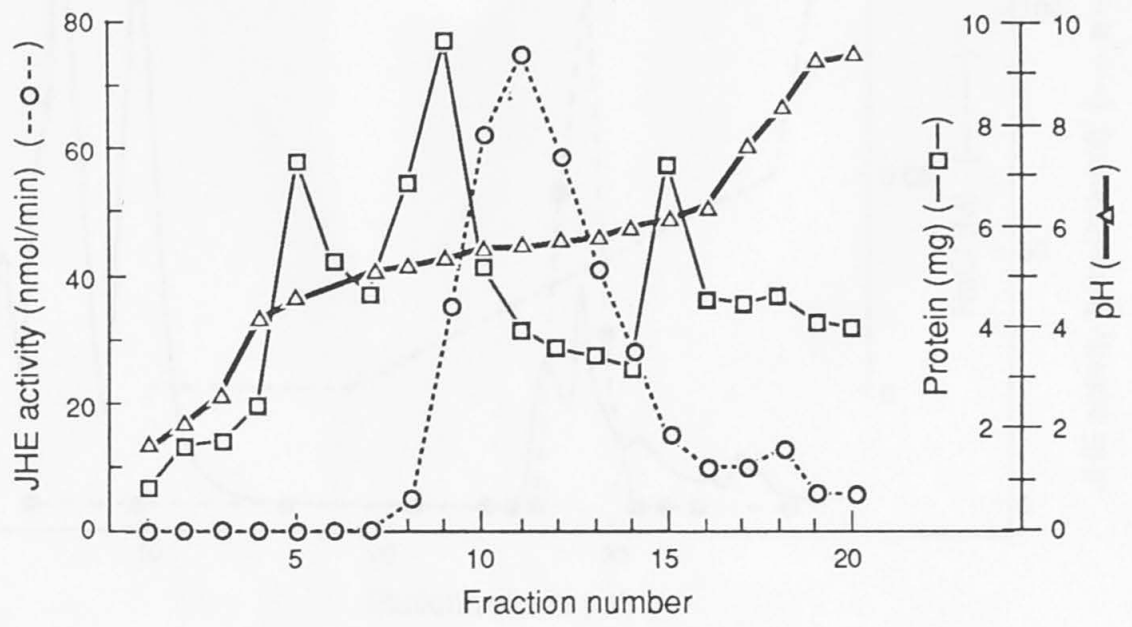

(B)

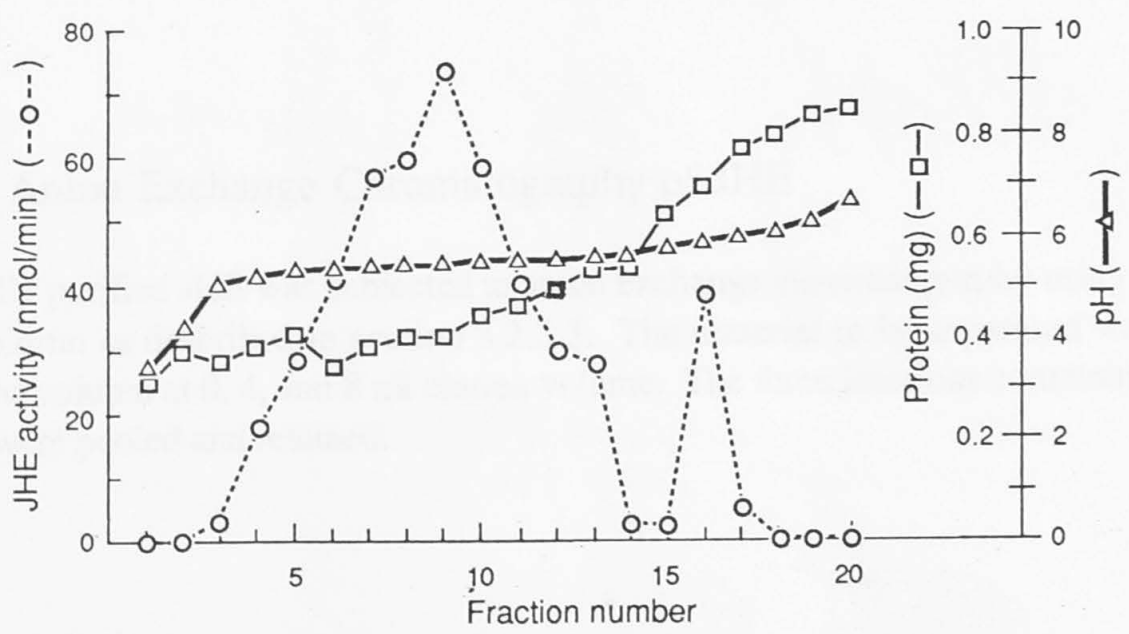

Figure 3.4 Preparative Isoelectric Focussing of JHE

(A) Partially purified JHE was subjected to isoelectric focussing using a "Rotofor" apparatus as described in section 3.2.3.3. (B) The fractions from (A) containing JHE activity (9-15) were pooled and refractionated. Fractions 5-13 were pooled and retained. The $\mathrm{JH}$ hydrolytic activity detected in fraction 16 was due to JHEH. 


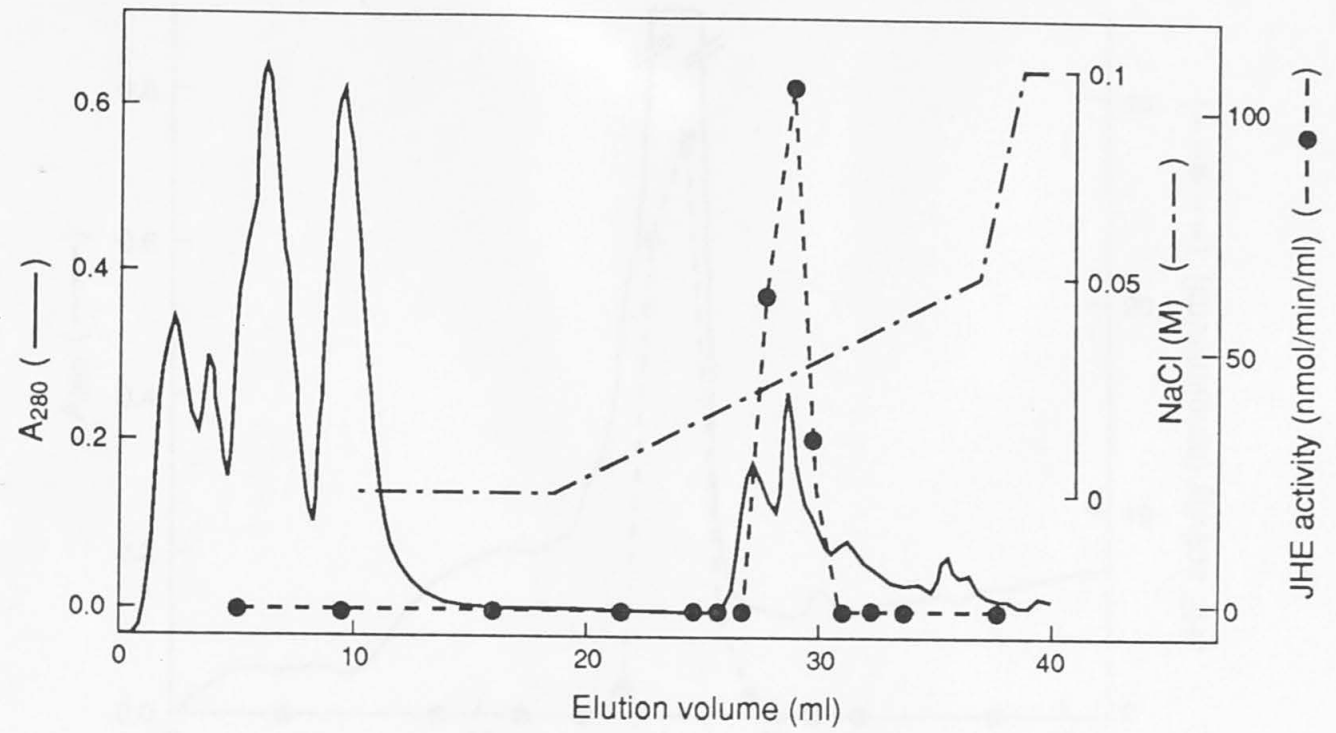

Figure 3.5 Anion Exchange Chromatography of JHE

Partially purified JHE was subjected to anion exchange chromatography using a "Mono Q" column as described in section 3.2.3.3. The material to be separated was loaded onto the column at 0,4 , and $8 \mathrm{ml}$ elution volume. The three fractions containing JHE activity were pooled and retained. 


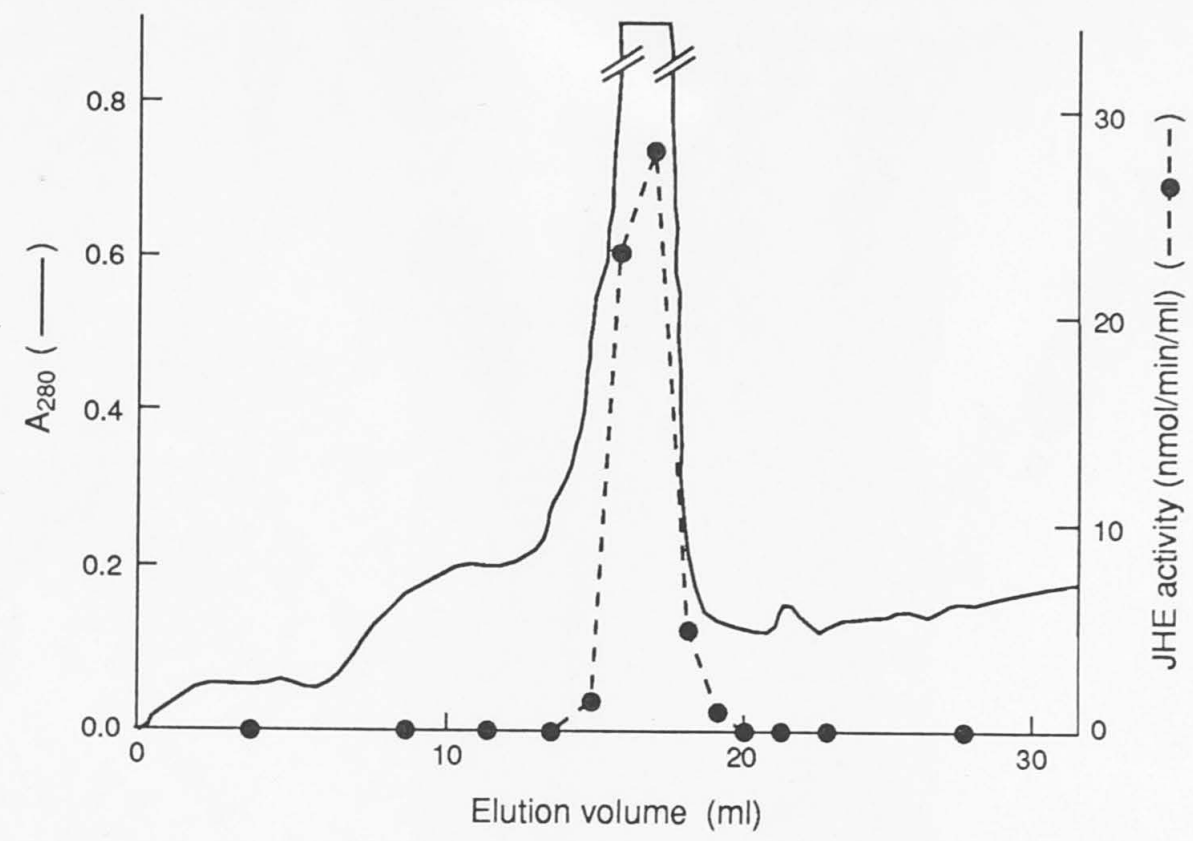

Figure 3.6 Gel Filtration of JHE

Partially purified JHE was subjected to gel filtration using a "Superose 6" column as described in section 3.2.3.3. The sixth and seventh fractions (Fr6 and Fr7) were retained. 
A

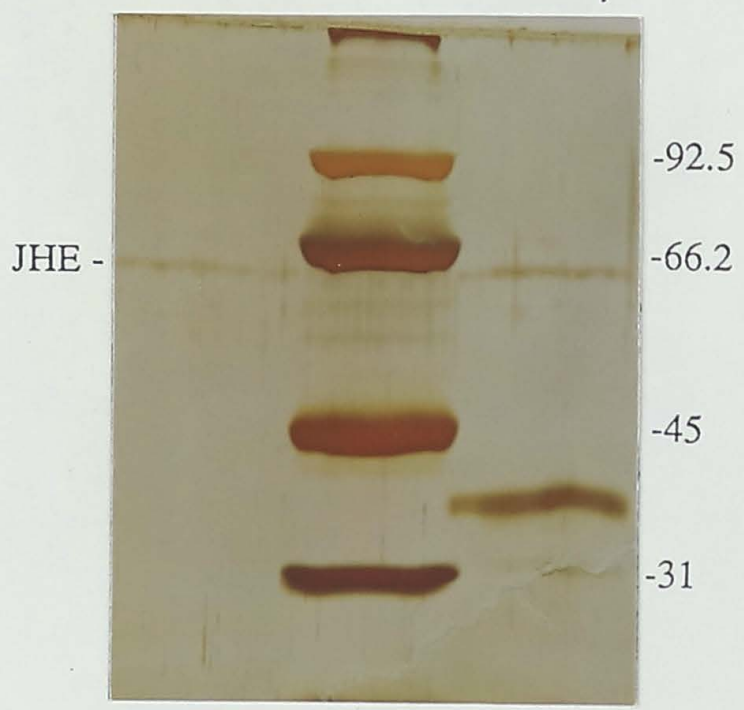

Fr6 MW Fr7

Figure 3.7 Analysis of Purified JHE by PAGE

(A) Purified JHE (Fr6 and Fr7) were separated by denaturing PAGE soon after purification and silver stained for total protein. The sizes of molecular weight marker proteins (MW) are indicated in $\mathrm{kDa}$.

(B) Aliquots of Fr7 were separated by non-denaturing PAGE in four lanes. Lane 1 was cut into pieces. The pieces were assayed for JHE activity and the position of the recovered enzyme is indicated. Lane 2 was silver stained for total protein. Lane 3 was incubated with $\alpha$ - and $\beta$-naphthylacetate to detect esterase activity. The brown stain indicates that JHE preferentially hydrolyses $\alpha$-naphthylacetate. Lane 4 was cut into pieces and a gel piece corresponding to the location of the $\alpha$-naphthylacetate and JH esterase activities was subjected to denaturing PAGE (shown in (C), Fr7 2nd D).

(C) Fr6 and Fr7 were separated by denaturing PAGE after storage for about one week at $-20^{\circ} \mathrm{C}$ and silver stained for total protein. 


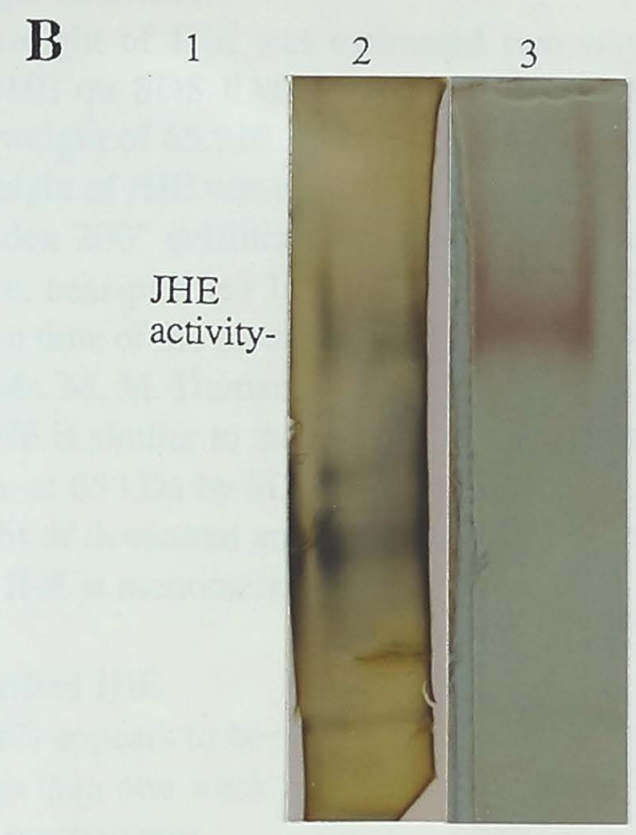

C

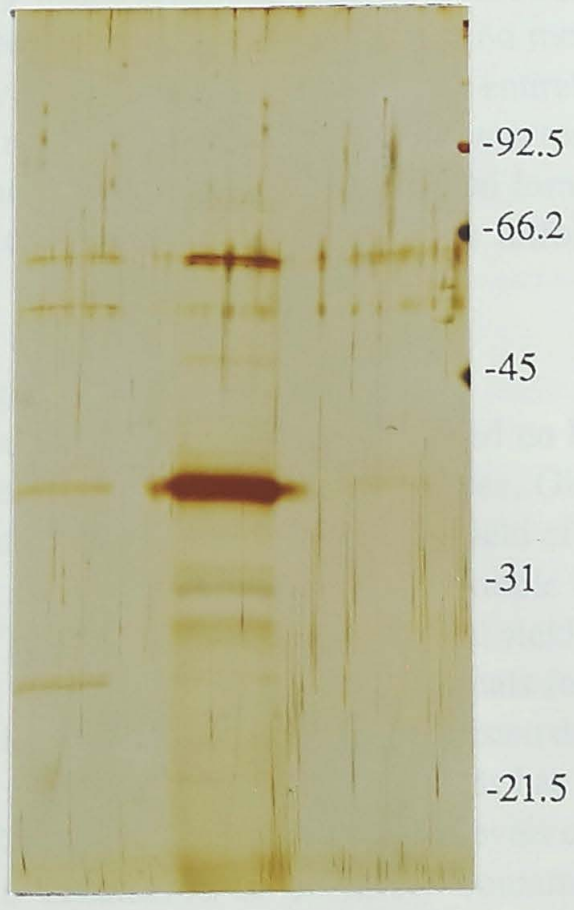

$\begin{array}{ccc}\text { Fr6 } & \text { Fr7 } & \text { Fr7 } \\ & \text { 2nd D }\end{array}$ 


\subsubsection{Molecular Weight Estimation}

The molecular weight of JHE was estimated two ways. Firstly, the relative mobility of purified JHE on SDS-PAGE compared with marker proteins (Biorad) indicated a molecular weight of $65.9 \pm 0.2 \mathrm{kDa}$ (SE from 4 determinations). Secondly, the native molecular weight of JHE was estimated to be $54 \mathrm{kDa}$ (1 determination) by use of a calibrated "Superdex 200" gelfiltration column (Pharmacia). Furthermore, the retention time of native, near-purified JHE on the Superose 6 column is only slightly longer than the retention time of the monomeric esterase, EST6 under similar conditions on the same column (Ms. M. M. Dumancic, unpublished data). This suggests that the molecular weight of JHE is similar to that of EST6 which is estimated to be $62 \mathrm{kDa}$ by calibrated gel filtration, or $65 \mathrm{kDa}$ by SDS-PAGE (Mane et al., 1983). Thus, estimates of the molecular weight of denatured and native JHE are in fair agreement, indicating that the native form of JHE is monomeric.

\subsubsection{Stability of Purified JHE}

Purified JHE ( $\mathrm{Fr} 7)$ appears to be stable for over a year when stored at $-70^{\circ} \mathrm{C}$ but loses all activity in less than one week at $-20^{\circ} \mathrm{C}$ (Fr6 and Fr7). JHE $\mathrm{A}_{\wedge}$ lose activity on storage by at least two mechanisms.

The first results in the loss of a fragment of about $6 \mathrm{kDa}$. Although Fr6 appeared homogeneous soon after its preparation (Fig. 3.7A), a sample of Fr6 stored for about one week at $-20^{\circ} \mathrm{C}$ revealed an additional $60 \mathrm{kDa}$ peptide (Fig. 3.7C) which stained with the same intensity as the $66 \mathrm{kDa}$ JHE band (a trace of the $40 \mathrm{kDa}$ peptide was also revealed by longer staining). Similarly, after storage at $-20^{\circ} \mathrm{C}, \mathrm{Fr} 7$ also gained the 60 $\mathrm{kDa}$ peptide, with relatively less staining of the $66 \mathrm{kDa}$ form of JHE. The reduction in the intensity of staining of JHE, together with the appearance of the $60 \mathrm{kDa}$ peptide, suggests that JHE was degraded by the loss of a $6 \mathrm{kDa}$ fragment.

The second mechanism by which $\mathrm{JHE}_{\Lambda} \mathrm{h}_{\mathrm{H}}$ (ose activity with storage has no effect on its molecular weight as estimated by SDS-PAGE. The second mechanism is inferred because loss of catalytic activity at $-20^{\circ} \mathrm{C}$ cannot be attributed entirely to the mechanism described above. Thus, while most of the original activity was lost from a sample of Fr7 stored at $-20^{\circ} \mathrm{C}$, most of the JHE remained in the $66 \mathrm{kDa}$ form (Fig. 3.7C). Loss of activity by this second mechanism presumably reflects unfolding of the native structure of JHE.

\subsubsection{N-Terminal Sequencing}

Ten rounds of the sequencing reaction were performed on Fr6 and yielded the following amino acid sequence: X, X, Gly, Glu, Val, Ile, Leu, Glu, Ala, X, where X indicates that the amino acid was not identified. The initial yield of the reaction was 70 pmoles, $23 \%$ of the amount of JHE estimated to be in the sample based on its protein content and molecular weight. The lower than expected initial yield may have been due to partial N-terminal blockage. However, high levels of signals for other amino acids were reported, suggesting that the low initial yield may have been due to degradation of JHE prior to sequencing. The sequence of seven amino acids has $100 \%$ identity with seven amino acids of plasmid R27 replication region, lower levels of similarity to other proteins, none of which were considered to be likely contaminants of the JHE preparation, and no similarity to other esterases. 


\subsubsection{Kinetic Characterisation of JHE}

Four experiments were performed using JHE conventionally purified to near homogeneity (Fr7, Section 3.3.1). In the first, kinetic constants for JHIII hydrolysis and constants for inhibition by three $\mathrm{JH}$ isoforms were determined. In the second, the kinetic properties of $\alpha$-naphthylacetate as both competitive inhibitor of JHIII hydrolysis and spectrophotometric substrate were determined. These first two experiments yielded kinetic parameters for a small range of alternative substrates. The third experiment yielded a ranking of the binding affinity of JHE for a much larger range of $\mathrm{JH}$ analogues and isoforms. In the fourth experiment, the mode of interaction between the $\mathrm{JH}$ carrier, lipophorin, and JHE was investigated.

\subsubsection{Kinetic Analysis of JHIII Hydrolysis and Its Inhibition by JH Isoforms}

The kinetic parameters for hydrolysis of racemic JHIII by D. melanogaster JHE are as follows (See figure 3.8 and the appendix for an example).

\begin{tabular}{ll}
\hline $\mathrm{K}_{\mathrm{M}}$ & $89 \pm 12 \mathrm{nM}$ (SE for 13 determinations) \\
$\mathrm{V}_{\max }$ & $590 \mathrm{nmol} / \mathrm{min} / \mathrm{mg}$ \\
$\mathrm{K}_{\text {cat }}$ & $0.60 \mathrm{sec}^{-1}$ \\
Specificity Constant $\left(\mathrm{K}_{\text {cat }} / \mathrm{K}_{\mathrm{M}}\right)$ & $6.8 \times 10^{6} \mathrm{M}^{-1} \mathrm{sec}^{-1}$ \\
\hline
\end{tabular}

These data indicate that the $\mathrm{V}_{\max } / \mathrm{K}_{\text {cat }}$ is quite low but both the binding affinity and specificity constant of $D$. melanogaster JHE for JHII are very high.

The kinetics of inhibition of JHIII hydrolysis by a selection of natural $\mathrm{JH}$ isoforms was investigated in order to determine whether JHE selectively binds a defined $\mathrm{JH}$ isoform. Each of these compounds exhibited competitive inhibition kinetics and no data set produced a significant fit to non-competitive inhibition kinetics. This indicates that $\mathrm{JH}$ isoforms bound to the same site as the substrate JHIII and that the $\mathrm{K}_{\mathrm{I}}$ can be interpreted as a measure of binding affinity of JHE for these compounds. Inhibition constants $\left(\mathrm{K}_{\mathrm{I}} \mathrm{s}\right)$ (weighted means of three determinations with standard errors) were as follows (see figure 3.8 and the appendix for an example).

\begin{tabular}{ll}
\hline $\mathrm{JHB}_{3}$ (optically racemic) & $1.20 \pm 0.09 \mu \mathrm{M}$ \\
$\mathrm{MF}$ & $3.33 \pm 0.36 \mu \mathrm{M}$ \\
$\mathrm{JHI}$ & $3.34 \pm 0.47 \mu \mathrm{M}$ \\
\hline
\end{tabular}

These $\mathrm{K}_{\mathrm{I}} \mathrm{s}$ are 13 to 38 fold higher than the $\mathrm{K}_{\mathrm{M}}$ for JHIII above although, consistent with its probable role in Diptera, the $\mathrm{K}_{\mathrm{I}}$ for the $\mathrm{JHB}_{3}$ is the lowest.

\subsubsection{Kinetic Properties with $\alpha$-Naphthylacetate}

The artificial substrate $\alpha$-naphthylacetate was shown to inhibit JHIII hydrolysis competitively with a $\mathrm{K}_{\mathrm{I}}$ of $120 \pm 30 \mu \mathrm{M}$ (weighted mean of 3 determinations with standard error) and no data set produced a significant fit to non-competitive inhibition kinetics. Hydrolysis of naphthylacetate by JHE was demonstrated after native PAGE and the alpha form is hydrolysed preferentially over the beta form (Section 3.3.2.1, Fig. 3.7B). The kinetics of hydrolysis of $\alpha$-naphthylacetate were also investigated spectrophotometrically (Fig. 3.9, Mastrapaolo and Yourno, 1981). These data were 


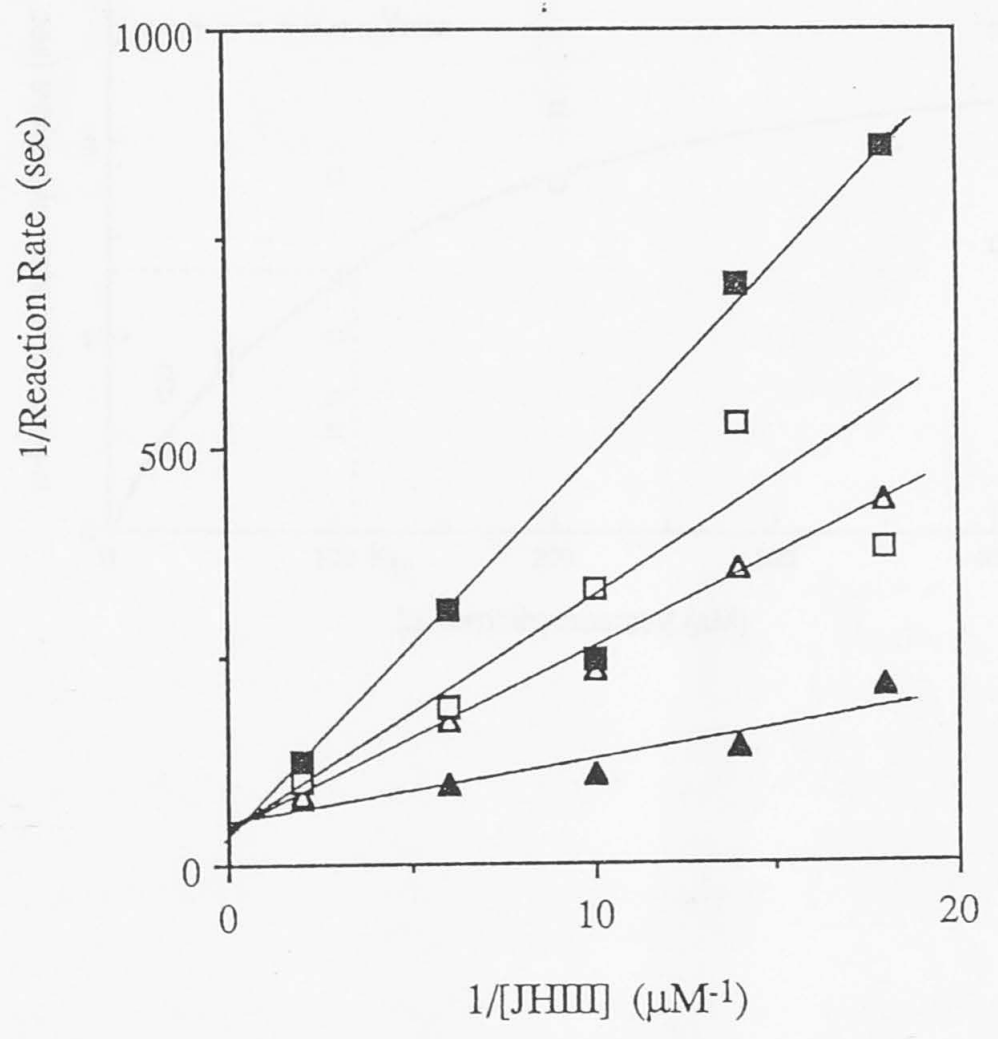

Figure 3.8 Inhibition of JHIII Hydrolysis by $\mathrm{JHB}_{3}: 1 / \mathrm{V}$ vs $1 /[\mathrm{S}]$ Plot

A typical experiment as described in section 3.2.4.1 to determine the nature of inhibition of JHIII hydrolysis, in this case by $\mathrm{JHB}_{3}$, is illustrated. $\mathrm{JHB}_{3}$ concentrations were zero (solid triangles), $3 \mu \mathrm{M}$ (open triangles), $5 \mu \mathrm{M}$ (open squares), and $8 \mu \mathrm{M}$ (solid squares). Similar results were obtained when MF, JHI and $\alpha$-naphthylacetate were tested as inhibitors of JHIII hydrolysis. 


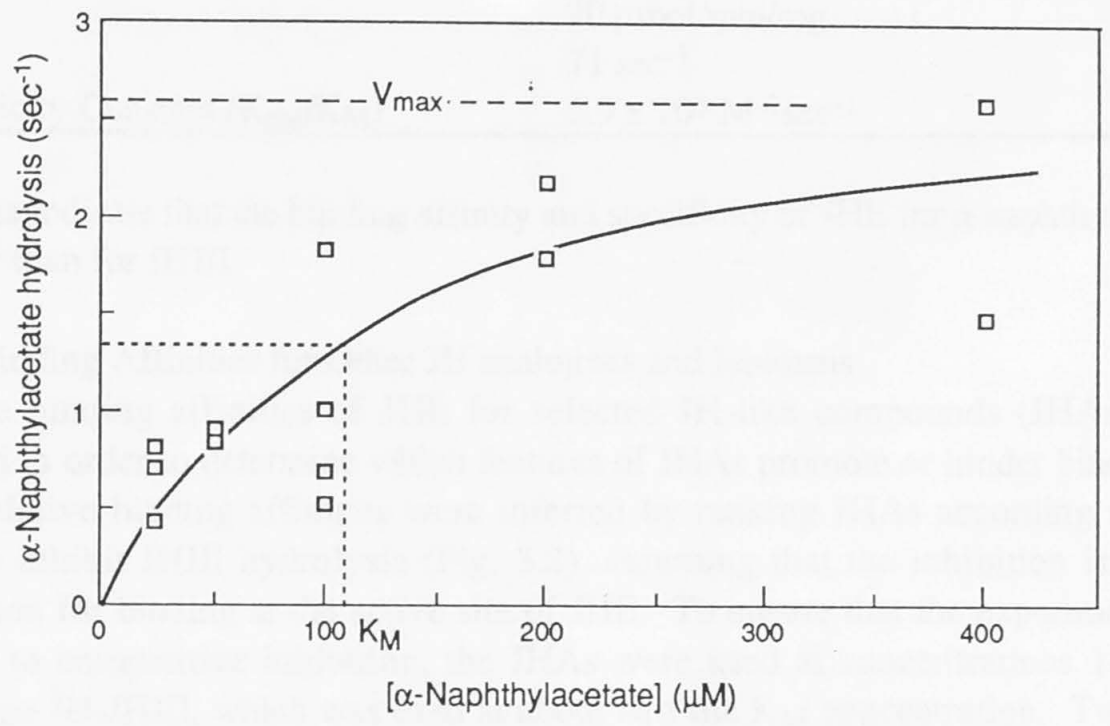

Figure 3.9 Hydrolysis of $\alpha$-Naphthylacetate by JHE: V vs [S] Plot

Hydrolysis of $\alpha$-naphthyacetate by JHE was monitored spectrophotometrically as described in section 3.2.4.2. The $\mathrm{V}_{\max }$ and $\mathrm{K}_{\mathrm{M}}$ for the reaction were estimated visually as indicated. 
not analysed statistically because of the obvious scatter but graphical analysis of the results indicate the following approximate kinetic parameters.

\begin{tabular}{ll}
\hline $\mathrm{K}_{\mathrm{M}}$ & $120 \mu \mathrm{M}$ \\
$\mathrm{V}_{\max }$ & $70 \mu \mathrm{mol} / \mathrm{min} / \mathrm{mg}$ \\
$\mathrm{K}_{\text {cat }}$ & $71 \mathrm{sec}^{-1}$ \\
Specificity Constant $\left(\mathrm{K}_{\text {cat }} / \mathrm{K}_{\mathrm{M}}\right)$ & $5.9 \times 10^{5} \mathrm{M}^{-1} \mathrm{sec}^{-1}$ \\
\hline
\end{tabular}

These data indicate that the binding affinity and specificity of JHE for $\alpha$-naphthylacetate are lower than for JHIII.

\subsubsection{Binding Affinities for Other JH analogues and Isoforms}

The binding affinities of JHE for selected JH-like compounds (JHAs) were compared in order to determine which features of JHAs promote or hinder binding by JHE. Relative binding affinities were inferred by ranking JHAs according to their ability to inhibit JHIII hydrolysis (Fig. 3.2), assuming that the inhibition is due to competition for binding at the active site of JHE. To ensure that the experiment was sensitive to competitive inhibition, the JHAs were used at concentrations 180 -fold higher than ${ }^{3} \mathrm{H}-\mathrm{JHIII}$, which was used at about half the $\mathrm{K}_{\mathrm{M}}$ concentration. Two lines of evidence support the assumption of competition. Firstly, $\alpha$-naphthylacetate binds at the same active site as JHIII (Section 3.3.3.2) and the JHAs are all more similar to JHIII than $\alpha$-naphthylacetate. Secondly, the relative abilities of JHI, JHIII, MF and $\mathrm{JHB}_{3}$ to inhibit JHIII hydrolysis are consistent with the inhibition/Michaelis constants calculated for these compounds (see below), and competitive inhibition by JHI, MF and $\mathrm{JHB}_{3}$ has been rigorously demonstrated (Section 3.3.3.1):

\begin{tabular}{lll}
\hline Compound & $\mathrm{K}_{\mathrm{I}} / \mathrm{K}_{\mathrm{M}} \pm \mathrm{SE}$ & \% Inhibition of JHIII hydrolysis $\pm \mathrm{SE}$ \\
\hline $\mathrm{JHI}$ & $3.34 \pm 0.47 \mu \mathrm{M}$ & $63.0 \pm 2.3 \%$ \\
$\mathrm{MF}$ & $3.33 \pm 0.36 \mu \mathrm{M}$ & $60.0 \pm 1.8 \%$ \\
$\mathrm{JHB}_{3}$ & $1.20 \pm 0.09 \mu \mathrm{M}$ & $85.5 \pm 0.4 \%$ \\
$\mathrm{JHIII}$ & $89 \pm 12 \mathrm{nM}$ & $92.7 \pm 0.4 \%$ \\
\hline
\end{tabular}

Thus, JHI and MF have equal $\mathrm{K}_{\mathrm{I}}$ s and were ranked as essentially the same in this experiment. $\mathrm{JHB}_{3}$ has a lower $\mathrm{K}_{\mathrm{I}}$ and caused greater inhibition, while JHIII, which has a $\mathrm{K}_{\mathrm{M}}$ lower still, caused even greater inhibition when tested as a JHA.

The effects of JHAs on JHII hydrolysis (Fig. 3.2) are here compared within sets of JHAs which share systematic differences from JHIII. The inhibition data are presented as means of triplicate experiments with standard errors.

1) Size of the carboxyl chain

The replacement of one or two of the methyl side chains of JHIII with ethyl groups produces the lepidopteran JH isoforms JHII or JHI, respectively. JHII (C11 ethyl group) has reduced ability to inhibit JHIII hydrolysis $(81.3 \pm 2.3 \%)$, compared with an equal amount of non-radioactive JHIII $(92.7 \pm 0.4 \%)$ and JHI (ethyl groups at $\mathrm{C} 11$ and $\mathrm{C} 7)$ shows a further reduction $(63.0 \pm 2.3 \%$, Fig. 3.10A). However, little inhibition occurs if the JHIII backbone is truncated to $2 / 3$ of its original length, as exemplified by methyl geranoate $(3.5 \pm 1.7 \%)$. These data suggest that JHE binds most 
effectively to molecules with carboxyl chains that are neither larger nor smaller than JHIII. In particular, the distal portion of the carboxyl chain which carries the epoxide group is required for high affinity binding.

\section{2) Alternative alcohol moities}

Replacement of the methyl alcohol moiety of JHIII with an ethyl group reduces inhibition only slightly compared with JHIII ( $82.1 \pm 1.2 \%$ and $92.7 \pm 0.4 \%$, repectively). However, replacement with a longer alcohol group (dodecyl ester of JHIII) or the absence of any alcohol moiety ( $\mathrm{JH}$ acid) gives essentially no inhibition $(0.6 \pm 3.9 \%$ and $1.6 \pm 3.1 \%$, respectively), indicating that a small alcohol moiety is an important requirement for high affinity binding (Fig. 3.10B).

\section{3) Hydrogenation of the double bonds in JHIII and methyl farnesoate}

The effect of hydrogenation of one or more double bonds was examined in JHIII and MF. Each of the four possible JHAs combining hydrogenation of the 2,3 and 6,7 double bonds of JHIII (Fig. 3.11A) and each of the nine possible JHAs combining hydrogenation of the three double bonds of MF (Fig. 3.11B) were tested for their ability to inhibit JHIII hydrolysis.

When an epoxide is present at carbons 10 and 11 (as in JHIII) hydrogenation of the 2,3 double bond reduces inhibition $\left(\mathrm{F}_{1,9}=131, \mathrm{p}<0.001\right)$ whereas hydrogenation of the 6,7 double bond increases inhibition $\left(\mathrm{F}_{1,9}=26, \mathrm{p}<0.001\right)$ and these effects are independent of one another $\left(F_{1,9}=4.7, p>0.05\right)$. On average 2,3 hydrogenation decreases inhibition by $17 \%$ but 6,7 hydrogenation increases inhibition by $8 \%$.

When there is no epoxide at carbons 10 and 11 (as in MF) the effects on inhibition of hydrogenation of the 2,3,6,7, and 10,11 double bonds are more difficult to assess because all three pairwise interactions, as well as the three way interaction, are significant (Fig. 3.11B). Nevertheless, inspection of the sums of squares reveals that the 2,3 and 6,7 bonds exert the largest of the three main effects, with hydrogenation causing overall decreases in percentage inhibition of 10 and 28 , respectively. By comparison, 10,11 hydrogenation has no independent (main) effect; instead its effects are manifest in the interaction terms, so its effects depend strongly on whether the 2,3 or 6,7 bonds are hydrogenated. When the 6,7 double bond is present, 10,11 hydrogenation increases percentage inhibition by 7 , but when the 6,7 double bond is hydrogenated, 10,11 hydrogenation decreases percentage inhibition by 13 . In contrast, when the 2,3 double bond is present, 10,11 hydrogenation decreases percentage inhibition by 24 , but when the 2,3 double bond is hydrogenated, 10,11 hydrogenation increases percentage inhibition by 14 .

The importance of the 2,3 double bond may be indicated by the absence of inhibition by the cis- 2 isomer of MF $(-2.4 \pm 5.3 \%)$. However, this modification probably causes the entire carboxyl chain to be displaced from its usual spatial relationship with the ester group.

Collating the results of these analyses, the presence of the 2,3 double bond promotes binding to JHE. The 6,7 double bond promotes binding if the 10,11 epoxide is absent but it reduces binding if the 10,11 epoxide is present. The greater inhibition by the entire JHIII-like (epoxidated) series compared with the MF-like series indicates that binding is more strongly promoted by the 10,11 epoxide group than by the presence or absence of any of the double bonds. 


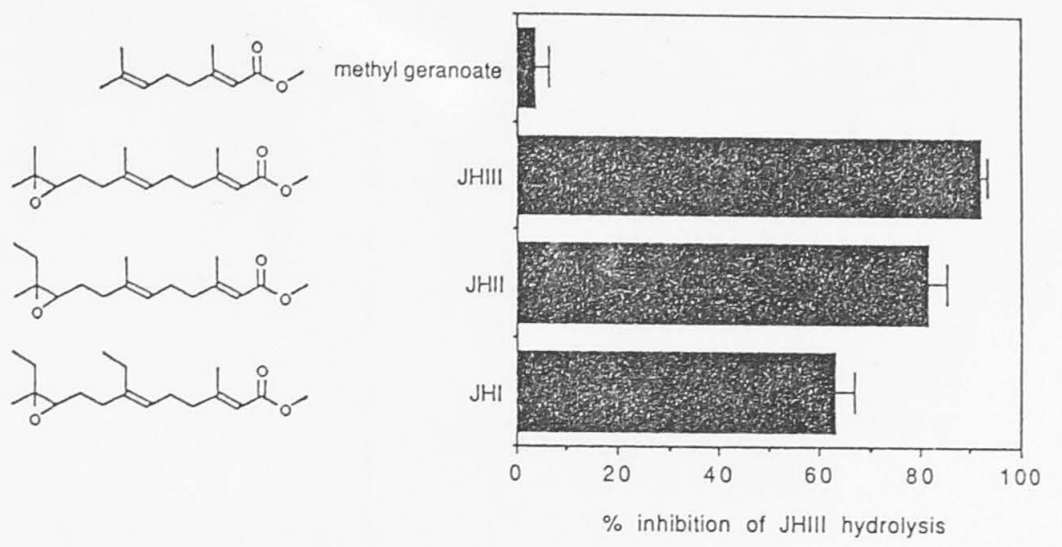

A

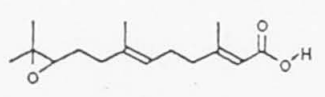

JHIllacid

B<smiles>CC(C)CCCC(C)CCCC(C)(C)C</smiles>

JHIII
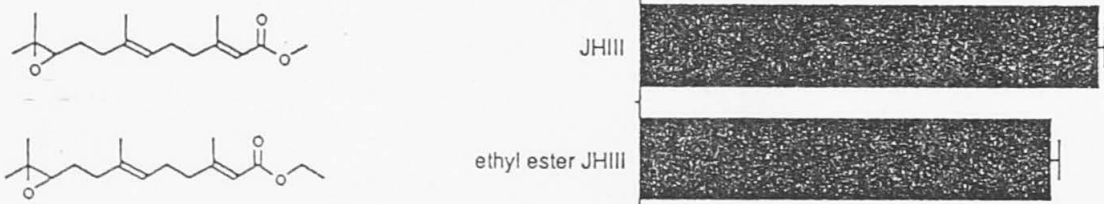

ethyl ester JHII
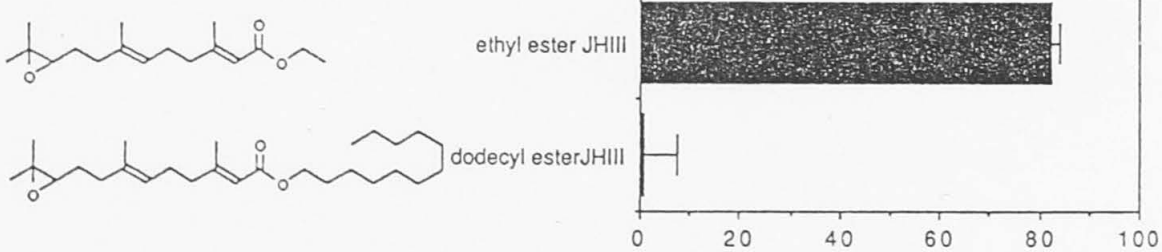

$\%$ inhibition of JHIII hydrolysis

Figure 3.10 Effects of JHAs on JHE hydrolysis of JHIII -Size of Carboxyl Chain and Alcohol Moiety

JHAs $(10 \mu \mathrm{M})$ were incubated with ${ }^{3} \mathrm{H}-\mathrm{JHIII}(56 \mathrm{nM})$ and JHE as described in section 3.2.4.3 and the \% inhibition of JHIII hydrolysis was calculated. (A) shows the effects by JHAs which differ in the size of the carboxyl chain and (B) shows the effects of JHAs which differ in the alcohol moiety. 
A

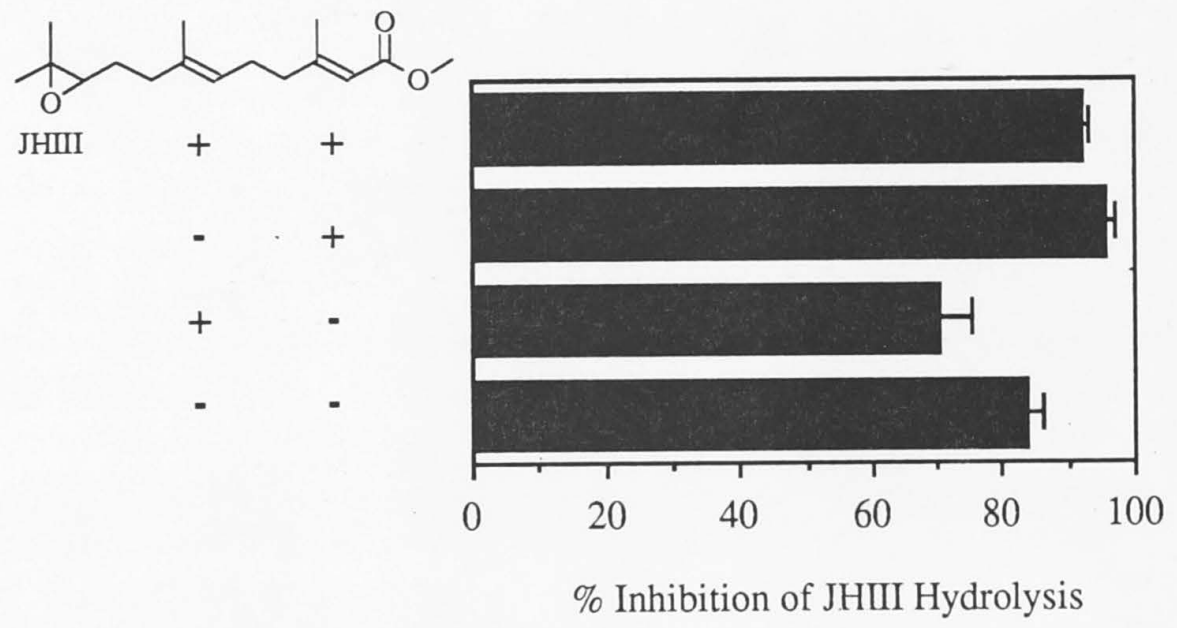

Figure 3.11 Effects of JHAs on JHE hydrolysis of JHIII -Effects of Double bonds

JHAs $(10 \mu \mathrm{M})$ were incubated with ${ }^{3} \mathrm{H}$-JHIII $(56 \mathrm{nM})$ and JHE as described in section 3.2.4.3 and the percent inhibition of JHIII hydrolysis was calculated. (A) shows the effects of JHAs which differ by the presence or absence of double bonds when the 10,11 epoxide is present, as in JHIII and (B) shows the effects of JHAs which differ by the presence or absence of double bonds when the 10,11 epoxide is absent, as in MF. The table shows the results of analysis of variance of the data shown in panel B. 


\section{B}

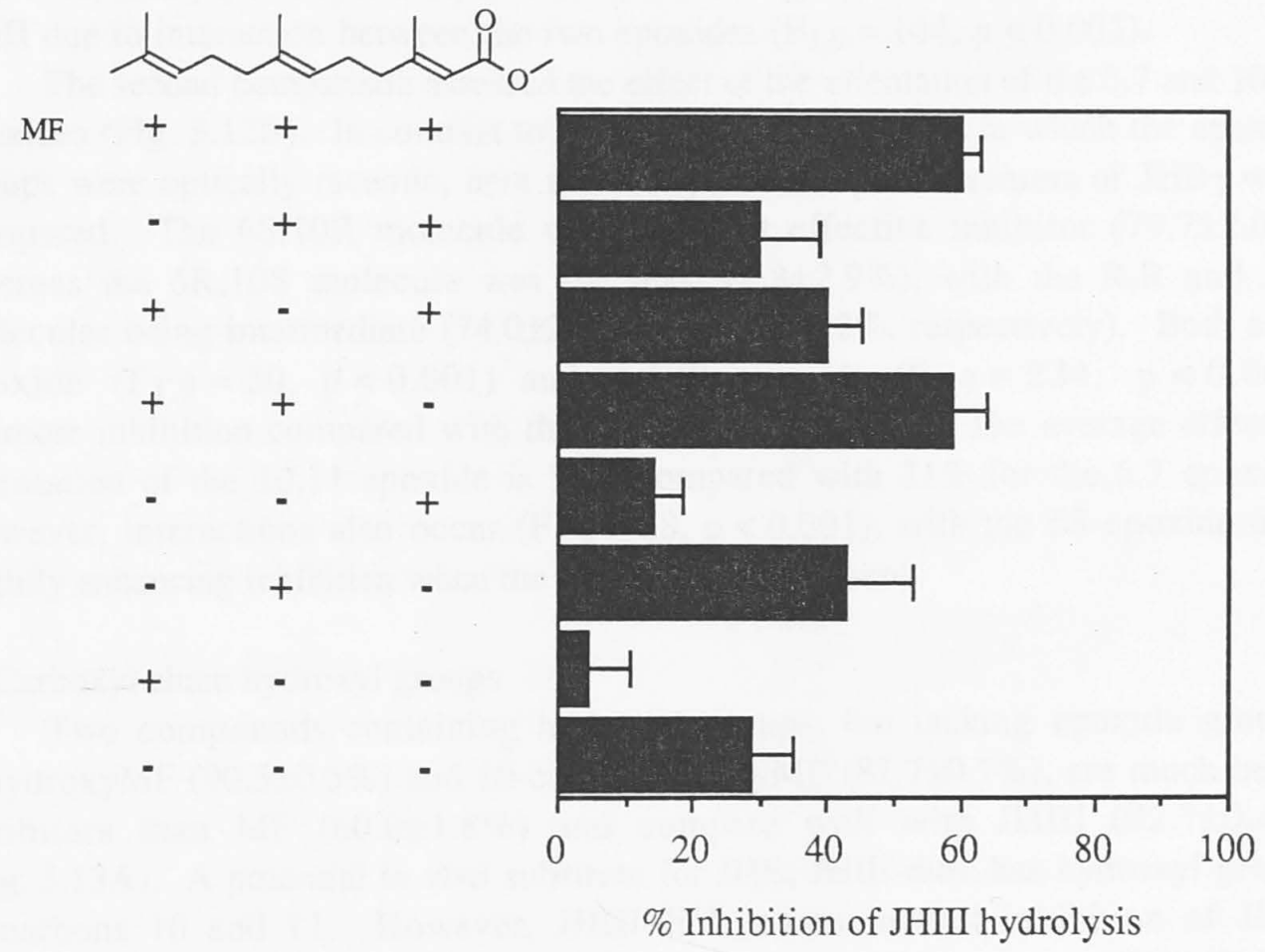

\begin{tabular}{lllcl}
\hline Double Bonds & Degrees of Freedom & Mean Square & F-test & \\
\hline 2,3 & 1 & 205 & 8 & $*$ \\
6,7 & 1 & 1857 & 71 & $* * *$ \\
10,11 & 1 & 45 & 1.7 & \\
$2,3 \times 6,7$ & 1 & 362 & 14 & $* *$ \\
$2,3 \times 10,11$ & 1 & 853 & 33 & $* * *$ \\
$6,7 \times 10,11$ & 1 & 249 & 10 & $* *$ \\
$2,3 \times 6,7 \times 10,11$ & 1 & 344 & 13 & $* *$ \\
\hline Error & 16 & 26 & & \\
\hline${ }^{*}<0.05,{ }^{* *} \mathrm{p}<0.01,{ }^{* * *} \mathrm{p}<0.001$ & & &
\end{tabular}


4) Alterations to the epoxide group(s)

Two types of comparison were used to assess the importance of epoxide groups in the binding of JH to JHE. Firstly, inhibition of JHIII hydrolysis was compared among the four possible JHAs which have either a double bond or an epoxide group between carbons 6 and 7 and carbons 10 and 11 (Fig. 3.12A). MF (no epoxides) was a poorer inhibitor $(60.0 \pm 1.8 \%)$ than either the 6,7 epoxide of $\mathrm{MF}(78.5 \pm 1.3 \%$ inhibition) or the 10,11 epoxide (JHIII, $92.7 \pm 0.4 \%$ ). Inhibition is promoted by both the 10,11 epoxide $\left(F_{1,9}=357, p<0.001\right)$ or the 6,7 epoxide $\left(F_{1,9}=11, p<0.05\right)$. However, $\mathrm{JHB}_{3}$ (both epoxides, $85.5 \pm 0.4 \%$ ) is a slightly less effective inhibitor than JHIII due to interaction between the two epoxides $\left(\mathrm{F}_{1,9}=144, \mathrm{p}<0.001\right)$.

The second comparison assessed the effect of the orientation of the 6,7 and 10,11 epoxides (Fig. 3.12B). In contrast to the previous comparisons in which the epoxide groups were optically racemic, here the four possible optical isomers of $\mathrm{JHB}_{3}$ were compared. The $6 \mathrm{~S}, 10 \mathrm{R}$ molecule was the most effective inhibitor (79.7 $\pm 2.0 \%)$ whereas the $6 \mathrm{R}, 10 \mathrm{~S}$ molecule was the least $(7.8 \pm 2.9 \%)$, with the R,R and $S, S$ molecules being intermediate $(74.0 \pm 2.0 \%$ and $44.6 \pm 3.2 \%$, respectively). Both a $6 \mathrm{~S}$ epoxide $\left(F_{1,9}=50, p<0.001\right)$ and a $10 \mathrm{R}$ epoxide $\left(F_{1,9}=234, p<0.001\right)$ promote inhibition compared with the opposite orientations. The average effect of orientation of the 10,11 epoxide is $51 \%$ compared with $21 \%$ for the 6,7 epoxide. However, interactions also occur $\left(\mathrm{F}_{1,9}=28, \mathrm{p}<0.001\right)$, with the $6 \mathrm{~S}$ epoxide only slightly enhancing inhibition when the $10 \mathrm{R}$ epoxide is present.

\section{5) Carboxyl chain hydroxyl groups}

Two compounds containing hydroxyl groups, but lacking epoxide groups, 8-hydroxyMF $(90.5 \pm 0.5 \%)$ and 10-cis,12-hydroxyMF $(81.7 \pm 0.7 \%)$, are much better inhibitors than MF $(60.0 \pm 1.8 \%)$ and compare well with JHIII $(92.7 \pm 0.4 \%)$ (Fig. 3.13A). A potential in vivo substrate for JHE, JHIII-diol, has hydroxyl groups at carbons 10 and 11. However, JHIII-diol causes modest inhibition of JHIII hydrolysis $(46.0 \pm 1.5 \%)$, indicating that it does not bind strongly to JHE. This is consistent with the finding that little or no $\mathrm{JH}$-acid-diol is produced when both JHE and JHEH are present (Section 2.3.2.2).

\section{6) Insecticidal JHAs}

The two JHAs tested that are registered insecticides were the juvenoids R/S-hydroprene and S-methoprene. These compounds mimic many of the effects of JH (Fig. 3.13B) but were poor inhibitors of $\mathrm{JH}$ hydrolysis $(10.0 \pm 3.1 \%$ and $0.0 \pm 3.7 \%$, respectively). Hydroprene and methoprene differ from JHIII by the presence of ethyl and isopropyl alcohol moities, respectively, the lack of an epoxide group, and the presence of an additional 4,5 double bond conjugated to the 2,3 double bond and the ester group. The first two features cause reduced inhibition of JHIII hydrolysis (Sections 3.3.3.3-2 and 3.3.3.3-4). $6 \mathrm{~S}-\mathrm{JHB}_{3}-2,3,4,5$-diene is a good inhibitor $(91.1 \pm 3.8 \%$ ) suggesting that the third feature, additional conjugation, does not interfere with binding to JHE, although it would be expected to increase the rigidity and stability of the molecule. Poor binding to JHE is consistent with slow metabolism of these juvenoids in a range of insects (Bowers, 1982). 


\subsubsection{Interaction of JHE with Lipophorin}

The double reciprocal plot of the velocity of JH hydrolysis versus substrate concentration yields a series of curves for reactions containing lipophorin rather than the straight line obtained in its absence (Fig. 3.14A). Rates of hydrolysis are reduced in the presence of lipophorin but it is clear that the $\mathrm{V}_{\max }$ of the reaction is unaltered. Furthermore, there is no difference between the rate of hydrolysis with and without lipophorin when the JHIII concentration $(5 \mu \mathrm{M})$ should be sufficiently high to saturate binding by both JHE and lipophorin $(0.6 \mu \mathrm{M})(\mathrm{p}=0.36, \mathrm{n}=3)$.

The free concentration of $\mathrm{JH}$ in the presence of lipophorin was calculated to test the hypothesis that the reduced rates of JH hydrolysis could be explained simply by reduced substrate concentration. In order to bind in the active site of JHE, a JH molecule presumably must not be bound in the binding site of lipophorin. The dissociation constant of lipophorin $(58 \mathrm{nM})$ and the concentrations of lipophorin and added $\mathrm{JH}$ were used to calculate the free concentrations of $\mathrm{JH}$ in the reactions, using the following formula.

$$
\left[\mathrm{L}_{\mathrm{T}}\right]=\mathrm{K}_{\mathrm{D}}(\mathrm{f} / 1-\mathrm{f})+\mathrm{f}\left[\mathrm{R}_{\mathrm{T}}\right] \quad \text { (Bennett and Yamamura, 1985) }
$$

$\mathrm{L}_{\mathrm{T}}$ is the total ligand concentration (JHIII), $\mathrm{R}_{\mathrm{T}}$ is the total receptor concentration (lipophorin), $\mathrm{K}_{\mathrm{D}}$ is its dissociation constant, $\mathrm{f}=[\mathrm{LR}] / \mathrm{R}_{\mathrm{T}}$, and $\mathrm{LR}$ is receptor bound to ligand. Solving the equations for [LR] yields the concentration of bound JHIII and free JHIII concentration is estimated by subtracting [LR] from $\mathrm{L}_{\mathrm{T}}$. Most of the calculated $\mathrm{JH}$ concentrations fell in the low nanomolar range and thus below the $\mathrm{JH}$ concentrations used in the reactions without lipophorin.

A double reciprocal plot using the calculated free JH concentrations (Fig. 3.14B) produced straight lines which lay close to the values predicted by extrapolation from the data collected in the absence of lipophorin. (Interestingly, these straight lines were fitted with correlation coefficients of 0.97-1.0 rather than the typical figure of 0.8-0.9, possibly due to reduced and less variable binding of JH to glassware). These results demonstrate that lipophorin does not inhibit JH hydrolysis by any mechanism other than by lowering the free concentration available to JHE.

Reaction rates for a single lower $\mathrm{JH}$ concentration $(7.2 \mathrm{nM})$ without lipophorin were somewhat lower than would be predicted by extrapolation from the data collected in the absence of lipophorin whereas the reaction rates determined with lipophorin were somewhat higher than would be predicted. These departures from linearity may be due to a higher proportion of non-specific binding of $\mathrm{JH}$ to the glassware when $\mathrm{JH}$ is very dilute and the ability of lipophorin to prevent this effect. Lanzrein et al. (1993) found that carbowax treatment of glassware, as used in these experiments, reduces surface binding of JHIII (from $78 \%$ bound to $44 \%$, for approx. 3.7 pM 10R-JHIII), but lipophorin and carbowax together eliminate such binding.

\subsection{Discussion}

\subsubsection{Purification and Physical Properties of JHE}

The first approach taken for the purification of $D$. melanogaster JHE was the affinity chromatography method of Abdel-Aal and Hammock (1986). Although JHE from $D$. melanogaster bound to the affinity matrices, successful application of this approach was prevented by the inability to pretreat starting material with DFP or recover 

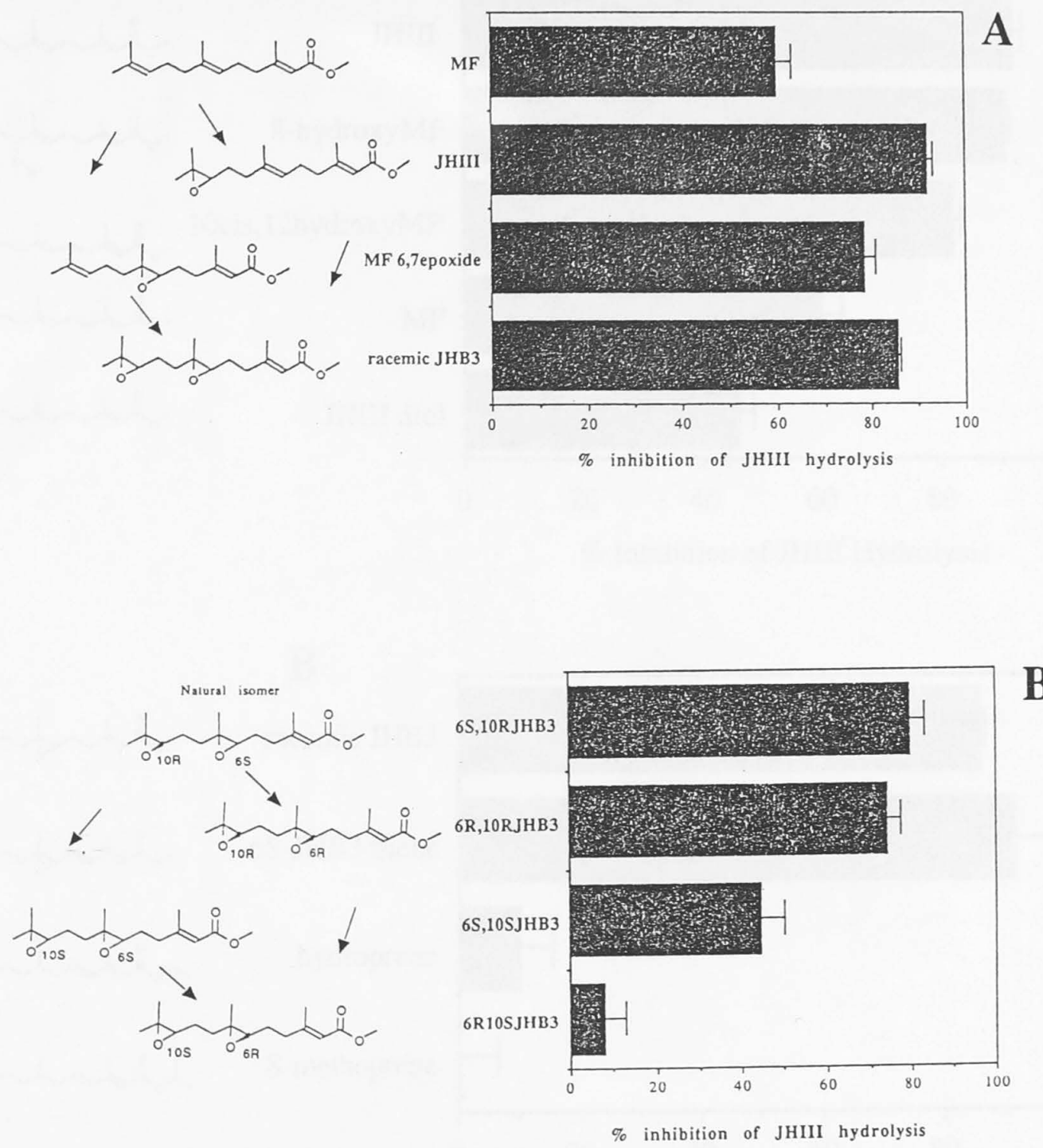

Figure 3.12 Effects of JHAs on JHE hydrolysis of JHIII -Effects of Epoxides

JHAs $(10 \mu \mathrm{M})$ were incubated with ${ }^{3} \mathrm{H}-\mathrm{JHIII}(56 \mathrm{nM})$ and JHE as described in section 3.2.4.3 and the percent inhibition of JHIII hydrolysis was calculated. (A) shows the effects of JHAs which differ by the presence or absence of 6,7 and 10,11 epoxide moieties and (B) shows the effects of JHAs which are optical isomers of $\mathrm{JHB}_{3}$. 

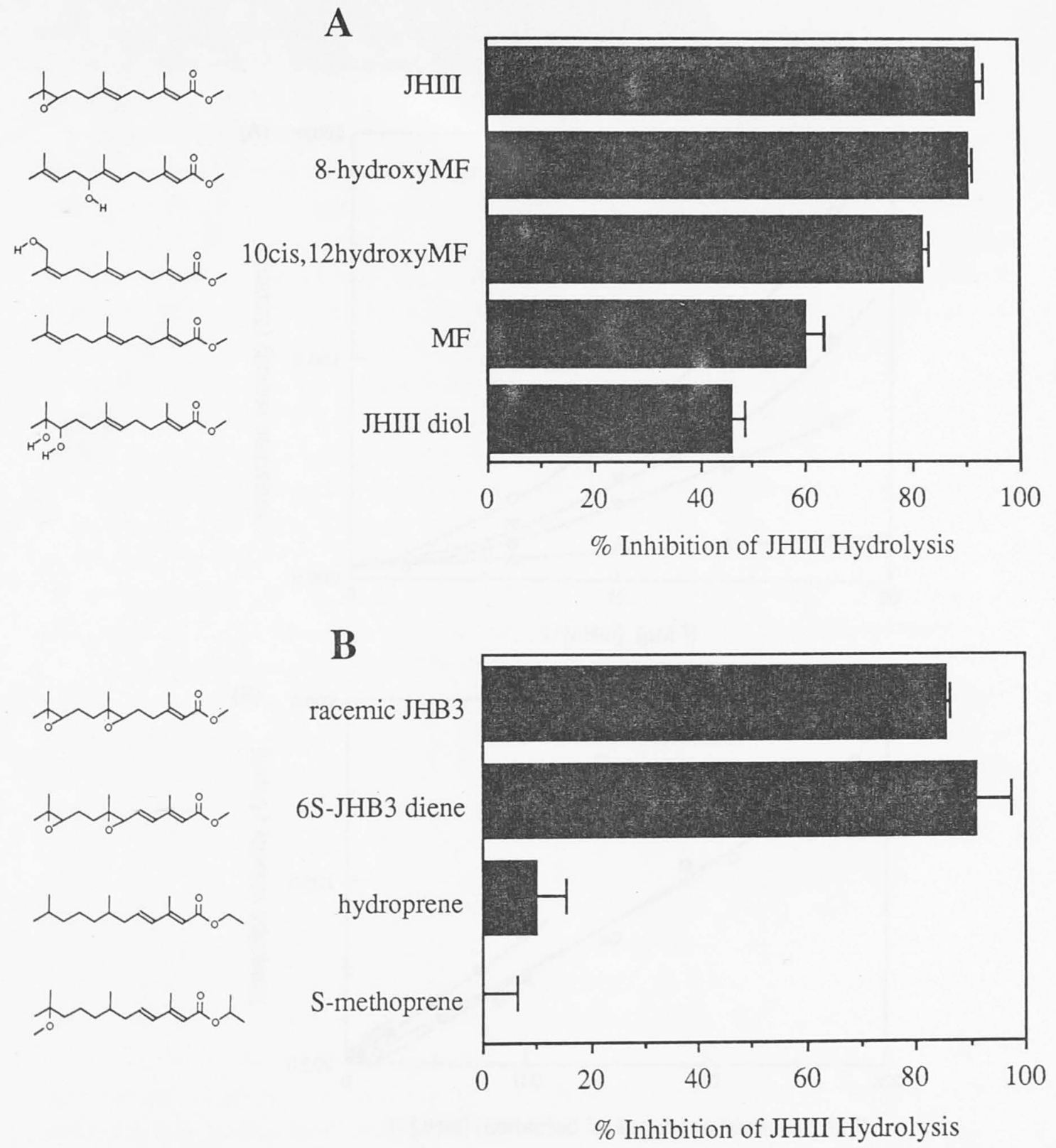

Figure 3.13 Effects of JHAs on JHE hydrolysis of JHIII -Hydroxyl Groups and Insecticidal JHAs

JHAs $(10 \mu \mathrm{M})$ were incubated with ${ }^{3} \mathrm{H}-\mathrm{JHIII}(56 \mathrm{nM})$ and JHE as described in section 3.2.4.3 and the percent inhibition of JHIII hydrolysis was calculated. (A) shows the effects by JHAs which have hydroxyl groups on the carboxyl chain and (B) shows the effects of two insecticidal JHAs compared with $\mathrm{JHB}_{3}$ and $\mathrm{JHB}_{3}$-diene. 

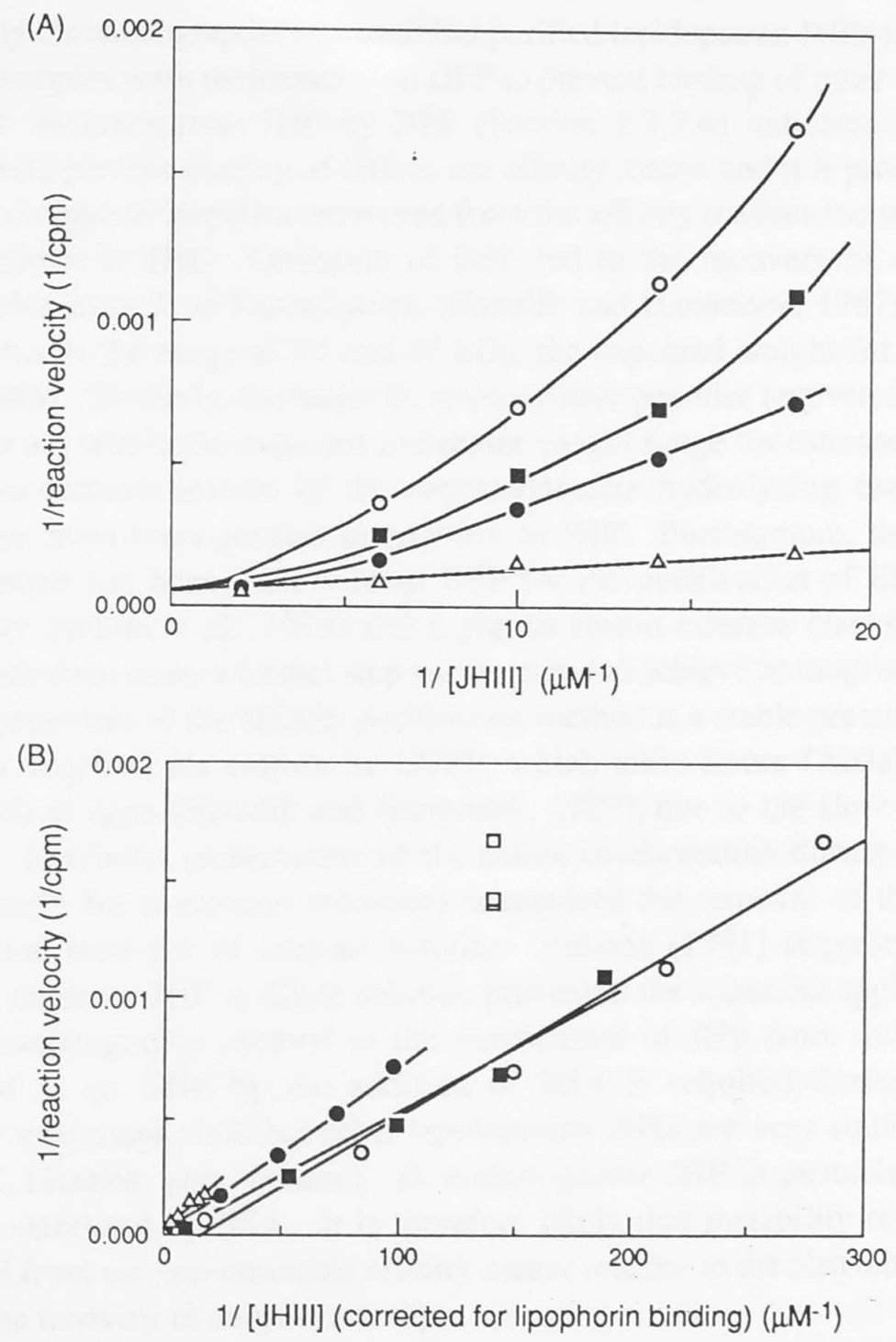

Figure 3.14 Inhibition of JHIII Hydrolysis by Lipophorin: 1/V vs 1/[S] Plot

Lipophorin was tested as an inhibitor of JHIII hydrolysis by JHE as described in section 3.2.4.4. Lipophorin concentrations were zero (open triangles), $0.1 \mathrm{mg} / \mathrm{ml}$ (solid circles), $0.2 \mathrm{mg} / \mathrm{ml}$ (solid squares), and $0.3 \mathrm{mg} / \mathrm{ml}$ (open circles). (A) shows the effect of lipophorin plotted against the concentration of added JHIII. (B) shows the same data plotted against the concentration of unbound JHIII which was calculated as described in section 3.3.3.4. In (B) the open squares indicate additional reactions which were performed without lipophorin. 
enzyme activity, probably due to instability of JHE in dilute solution. The second approach used classical chromatographic techniques to purifiy $D$. melanogaster JHE to homogeneity.

The affinity chromatographic approach has purified lepidopteran JHEs in a single step only when samples were pretreated with DFP to prevent binding of other esterases. Inhibition of D. melanogaster JHE by DFP (Section 2.3.2.4) indicates that DFP pretreatment would prevent binding of JHE to the affinity matrix and it is probable that the major $D$. melanogaster peptides recovered from the affinity matices included other esterases in addition to JHE. Omission of DFP led to the recovery of about six additional peptides from T. $n i$ haemolymph (Hanzlik and Hammock, 1987) that had molecular weights in the range of 54 and $67 \mathrm{kDa}$, the expected weight for esterases (Myers et al., 1988). Similarly, the major D. melanogaster peptides recovered from the affinity matrices are within the expected molecular weight range for esterases and the affinity matrices remove several of the naphthylacetate hydrolysing esterases of D. melanogaster from homogenates in addition to JHE. Furthermore, the affinity purification method has been used without DFP for the purification of EST6 from D. melanogaster (White et al., 1988) and a pigeon serum esterase (Jackson et al., 1992), and in both these cases a further step was required to achieve homogeneity.

A key requirement of the affinity purification method is a stable protein. Native conformation is required for elution by OTFP, which takes hours (Abdel-Aal and Hammock, 1985) or days (Hanzlik and Hammock, 1987), due to the slow exchange rate of ligands. Similarly, preservation of the native conformation during extensive dialysis (1-2 weeks for maximum recovery) is required for removal of the eluting ligand, OTFP and recovery of enzyme activity. Valaitis (1991) suggests that the instability of L. maderae JHE in dilute solution prevented the sucessful application of the affinity chromatography method to the purification of JHE from this species. Stabilisation of $T . n i$ JHE by the addition of BSA is required during affinity purification for maximum yield but other lepidopteran JHEs are very stable without BSA (Dr. T. N. Hanzlik, pers. comm.). D. melanogaster JHE is unstable in dilute solution and is stabilised by BSA. It is therefore likely that instability reduced the recovery of JHE from the non-cleavable affinity matrix relative to the cleavable version and prevented the recovery of enzyme activity.

In the second purification approach, classical chromatographic procedures were successfully applied to JHE from whole D. melanogaster pupae. After six steps, D. melanogaster JHE was homogeneous by the criterion of a single peptide $(66 \mathrm{kDa})$ being visible after SDS-PAGE and silver staining for total protein. Furthermore, two dimensional PAGE revealed that JHE activity comigrated with the $66 \mathrm{kDa}$ peptide.

The recovery of JHE from this procedure $(0.64 \%$ for Fr6, or $1.4 \%$ for Fr6 plus Fr7) is within the range of recoveries of JHE by conventional purifications from lepidopteran haemolymph (1-40\%, Section 1.7.2) but about 10 fold less than was achieved by the affinity chromatography method with whole $T$. $n i$ larvae (10\%, Hanzlik and Hammock, 1987). The enrichment factor for D. melanogaster JHE (Fr6, 429 fold) also falls within the range of conventional purifications of lepidopteran JHEs (383-4000 fold), although the starting material for the latter purifications was haemolymph. The specific activity of the $D$. melanogaster JHE $(0.59 \mu \mathrm{mol} / \mathrm{min} / \mathrm{mg})$ falls at the lower end of the range for lepidopteran JHEs $(0.48-4 \mu \mathrm{mol} / \mathrm{min} / \mathrm{mg})$. However, the specific activity for the $D$. melanogaster enzyme is probably an underestimate because of the large losses that occurred in the final purification step. Such losses also occurred during 
a similar stage in the purification of L. maderae JHE (Valaitis, 1991) but an improved procedure which did not require gel filtration produced L. maderae JHE with 3.5 fold greater specific activity (Valaitis, 1992).

Although analytical IEF had revealed only one form of JHE in D. melanogaster pupae (Section 2.3.2.4), it remained possible that different forms might separate during chromatography. However, a single peak of JHE activity was recovered at each chromatographic step, providing no evidence of multiple forms. Multiple forms of JHE that occur in some other insects generally copurify and are only separated by isoelectric focussing (Section 1.7.4). The only exception is the use of concanvalin A-sepharose chromatography to separate unglycosylated and glycosylated forms of JHE from lepidopteran fat body, and these forms probably represent the unprocessed and mature JHE (Wozniak and Jones, 1990; Valaitis, 1992).

\subsubsection{Comparison of D. melanogaster JHE with Other Esterases}

Several lines of evidence suggest that $D$. melanogaster JHE is likely to be a member of the carboxyl/choline esterase multigene family (Section 1.8), although none of these provide sufficient evidence on their own. Firstly, its molecular weight (66 $\mathrm{kDa}$ ) is similar to the weights of the lepidopteran JHEs and other esterases. Secondly, it is inhibited by OTFP and DFP which inhibit other members of this family and suggest that it has an active site serine. Thirdly, it hydrolyses carboxylester substrates, and lastly, JHE from $H$. virescens has been shown by sequence comparison to be a member of this family.

The $\mathrm{N}$-terminal sequence of $D$. melanogaster JHE (7 residues) is not similar to any of the three published $\mathrm{N}$-terminal sequences of lepidopteran JHEs $(H$. virescens, complete cDNA sequence, Hanzlik et al., 1989; $M$. sexta, 15 residues, Venkatesh et al., 1990; L. dispar, 69 residues including a possible insertion of 22-24 amino acids, Valaitis, 1992). However, there is no more similarity in this region among the lepidopteran JHE sequences than among the JHEs and other esterases or among other esterases (15-30\%, Valaitis, 1992).

The conversion of purified D. melanogaster JHE from a $66 \mathrm{kDa}$ peptide to a 60 $\mathrm{kDa}$ peptide may be similar to the single cleavage event observed for Acetylcholinesterase (AChE). Disulphide reduction of purified AChEs from D. melanogaster $(70 \mathrm{kDa})$ yields a $55 \mathrm{kDa}$ peptide as well as a $16 \mathrm{kDa}$ peptide which contains the $\mathrm{N}$-terminus of the protein. House fly AChE has similar sized fragments and the cleavage is believed to occur in vivo, or during purification (Fournier et al., 1988). Similarly, AChE purified from vertebrates undergoes cleavage during storage. Therefore it has been proposed that the $75 \mathrm{kDa} A C h E$ protein of vertebrates is an inactive precursor of a protease which undergoes autolysis to a $25 \mathrm{kDa}$ fragment with trypsin-like activity and a $50 \mathrm{kDa}$ fragment which has carboxypeptidase activity and contains the AChE active site serine (Small, 1990). While the association of protease activity with AChE remains controversial (Wright et al., 1993), it is an interesting possibility that JHE also has protease activity which causes the loss of a $6 \mathrm{kDa}$ fragment during storage. However, such cleavage has not been observed in any other JHEs. 


\subsubsection{Kinetic Characterisation of JHE}

\subsubsection{JH-Specificity of JHE}

Kinetic characterisation of JHE was carried out to determine whether JHE satisfied biochemical criteria for JH-specificity. A "JH-specific esterase" has been defined as an enzyme which has a low $\mathrm{K}_{\mathrm{M}}$ for $\mathrm{JH}$, hydrolyses $\mathrm{JH}$ with a high $\mathrm{K}_{\mathrm{cat}} / \mathrm{K}_{\mathrm{M}}$ ratio, or, most rigorously, exhibits a higher $\mathrm{K}_{\mathrm{cat}} / \mathrm{K}_{\mathrm{M}}$ ratio for $\mathrm{JH}$ than for an alternative substrate such as $\alpha$-naphthylacetate (Section 1.5.3.1). The kinetic properties of the JH hydrolysing esterase purified from D. melanogaster in this study conforms to each of these criteria, indicating that this enzyme is indeed $\mathrm{JH}$-specific.

The specific activity of D. melanogaster JHE (Fr6, at least $0.59 \mu \mathrm{mol} / \mathrm{min} / \mathrm{mg}$ ) indicates a low $\mathrm{K}_{\text {cat }}$ for JHIII (at least $0.61 \mathrm{sec}^{-1}$ ). However, the specificity constant $\left(\mathrm{K}_{\mathrm{cat}} / \mathrm{K}_{\mathrm{M}}\right)$ of D. melanogaster JHE for JHIII is high (at least $6 \times 10^{6} \mathrm{M}^{-1} \mathrm{sec}^{-1}$ which approaches the diffusion limited maximum value of $10^{8}-10^{9} \mathrm{M}^{-1} \mathrm{sec}^{-1}$, Stryer, 1981) due to the very low $\mathrm{K}_{\mathrm{M}}(89 \mathrm{nM})$. The high specificity constant and low $\mathrm{K}_{\mathrm{M}}$ for JHIII indicate that this enzyme is well adapted to the task of scavenging trace concentrations of $\mathrm{JH}$ as was proposed in section 2.4.2.2. These kinetic parameters for D. melanogaster JHE are within the range for lepidopteran JHEs (Wing et al., 1984; Table 3.2). The high specificity constants indicate that both the $D$. melanogaster and lepidopteran esterases are specific for $\mathrm{JH}$ and the similar, low $\mathrm{K}_{\mathrm{M}}$ s suggest that they encounter similar, low JH concentrations in vivo.

Comparison of the kinetic parameters for hydrolysis of JHIII and the structurally different, artificial substrate, $\alpha$-naphthylacetate, confirm the specificity of D. melanogaster JHE for JH. Naphthylacetate inhibition of JHIII hydrolysis is competitive, and both the $\mathrm{K}_{\mathrm{M}}$ and $\mathrm{K}_{\mathrm{I}}$ values are $120 \mu \mathrm{M}$. These results demonstrate that both JHE and $\alpha$-naphthylacetate are hydrolysed by the same active site. Although JHE can hydrolyse $\alpha$-naphthylacetate at a higher rate $(70 \mu \mathrm{mol} / \mathrm{min} / \mathrm{mg})$ than JHIII $(0.59 \mu \mathrm{mol} / \mathrm{min} / \mathrm{mg})$, the $\mathrm{K}_{\mathrm{M}}$ of JHE for $\alpha$-naphthylacetate is much higher than for JHIII and consequently the specificity constant $\left(5.9 \times 10^{5} \mathrm{M}^{-1} \mathrm{sec}^{-1}\right)$ is 12 fold lower than for JHIII. $H$. virescens JHE is the only other example for which kinetic parameters have been determined for the hydrolysis of an artificial substrate, in this case a thioester, $\mathrm{C}_{6} \mathrm{H}_{13} \mathrm{OCH}_{2} \mathrm{C}(\mathrm{O}) \mathrm{SCH}_{3}$ (Ward et al., 1992). In common with the $D$. melanogaster $\mathrm{JHE}$, the $H$. virescens $\mathrm{JHE}$ has a higher $\mathrm{K}_{\text {cat }}$ for the artificial substrate compared with JHIII, a much higher $\mathrm{K}_{\mathrm{M}}$, and consequently a lower specificity constant (Table 3.2). Although the higher rates of hydrolysis with artificial substrates may seem counter-intuitive they can be explained because high binding affinity for the natural substrate is not compatible with rapid hydrolysis.

An additional criterion for the JH-specificity of JHE is its ability to hydrolysis $\mathrm{JH}$ in the presence of the JH carrier (Section 1.5.3.1). This criterion was proposed primarily because it is indicative of a low $\mathrm{K}_{\mathrm{M}}$ but it also relates to in vivo hydrolysis, a major component of which, at least in Lepidoptera, occurs in the presence of high affinity carriers in the haemolymph. A non-specific esterase with a fortuitous ability to hydrolyse $\mathrm{JH}$ would be expected to hydrolyse $\mathrm{JH}$ only in the absence of competition for JH binding by a specific carrier (Sanburg et al., 1975). The D. melanogaster JHE hydrolyses $\mathrm{JH}$ in the presence of the dipteran $\mathrm{JH}$ carrier, lipophorin, although the carrier comes from another species, L. cuprina. However, it is unclear whether coincubation of JHE and lipophorin can be used to model their interaction in vivo because it is unknown whether they occur in the same compartment. Lipophorin and JHE may be 
located together in the haemolymph of dipteran pupae but in larvae and adults the $\mathrm{JH}$ degrading enzymes appear to be located in non-haemolymph tissues (Section 1.6.4).

\subsubsection{Characterisation of Substrate Binding by JHE}

Analysis of inhibition of JHIII hydrolysis by JH isoforms and analogues reveals that almost all deviations from the structure of JHIII reduce the affinity of binding to JHE. The overall size of the molecule is important and even small increases in size, such as the changes from methyl to ethyl side chains found in JHI and JHII, or increases in the size of the methyl ester moiety, decrease binding. While these results might suggest that molecules larger than JHIII are sterically hindered from binding in the active site, esters with larger alcohol moities are not absolutely precluded from hydrolysis, as demonstrated by the rapid hydrolysis of naphthylacetate. Conjugation of the 2,3 double bond with the ester group and the 10,11 epoxide in the $\mathrm{R}$ conformation both promote binding to JHE, consistent with all naturally occurring insect JHs having these features (Kindle et al., 1989; Hearlt et al., 1993). The strongest effect is promotion of binding by the $10 \mathrm{R}, 11$ epoxide but the results are equivocal with regard to the features at carbons 6 and 7 (discussed below).

Among the natural JH isoforms, D. melanogaster JHE has a higher affinity for JHIII and $\mathrm{JHB}_{3}$ (which may have hormonal roles in higher Diptera) than for JHI and MF (which are probably not dipteran hormones). The affinity of $D$. hydei JHE for JHAs also has been investigated, with compounds tested as inhibitors of JHI hydrolysis by homogenates of larval integument (Bisser and Emmerich, 1981). The $\mathrm{K}_{\mathrm{M}}$ of D. hydei JHE for JHI is $640 \mathrm{nM}$ and the data suggest a similar affinity for JHIII. This contrasts with D. melanogaster JHE which has a seven-fold lower $\mathrm{K}_{\mathrm{M}}$ for JHIII and a five-fold higher $\mathrm{K}_{\mathrm{I}}$ for JHI. D. hydei JHE has a greater affinity for the JHIII ethyl ester than for JHIII, also in contrast with D. melanogaster JHE. These differences might indicate that the binding site of $D$. hydei JHE is more tolerant of bulk both on the carboxyl chain and the alcohol moiety. Alternatively, the affinity of $D$. hydei JHE for JHIII relative to the other compounds may have been underestimated if the homogenates contained $\mathrm{JH}$ binding proteins with high affinity and specificity for JHIII. Both the D. melanogaster and $D$. hydei JHEs exhibit more than ten-fold lower affinity for MF than for JHIII, suggesting that binding by both enzymes is strongly promoted by the 10,11 epoxide. This conclusion is supported by the low affinity of both enzymes for methoprene.

An in vivo role for JHIII is suggested by the 13 fold lower $\mathrm{K}_{\mathrm{M}}$ of D. melanogaster JHE for JHIII than its $\mathrm{K}_{\mathrm{I}}$ for $\mathrm{JHB}_{3}$. This is not consistent with in vitro biosynthetic studies which suggest that $\mathrm{JHB}_{3}$ may be the only functional isoform in the higher Diptera (Section 1.2). However, the JHIII used in these experiments was a racemate of two optical isomers while the $\mathrm{JHB}_{3}$ was a racemate of four optical isomers. Thus, the greater dilution of the natural isomer of $\mathrm{JHB}_{3}$ with unnatural isomers may have exaggerated the difference in binding affinity between the natural isomers of $\mathrm{JHIII}$ and $\mathrm{JHB}_{3}$.

The data for modifications to the 6,7 bond are more difficult to interpret because the order in which modifications to the 6,7 bond promote binding is the reverse from expectations based on biosynthesis data. Thus, when the 10,11 epoxide is present (as in all insect JHs), a 6,7 double bond (as in JHIII) reduces binding affinity for JHE compared with a 6,7 single bond and the 6,7 epoxide (as in $\mathrm{JHB}_{3}$ ) further reduces binding affinity. Nevertheless, the correct, $6 \mathrm{~S}$ optical isomer of the 6,7 epoxide is 
Table 3.2. Comparison of Kinetic Parameters for Purified JHEs with JH Isoforms and Artificial Substrates.

\begin{tabular}{|c|c|c|c|c|c|c|}
\hline Species & Substrate & $\begin{array}{l}\mathrm{K}_{\mathrm{M}} \\
(\mathrm{nM})\end{array}$ & $\begin{array}{l}\mathrm{V}_{\max } \\
(\mu \mathrm{mol} / \mathrm{min} / \mathrm{mg})\end{array}$ & $\begin{array}{l}\mathrm{K}_{\text {cat }} \\
\left(\sec ^{-1}\right)\end{array}$ & $\begin{array}{l}\mathrm{K}_{\text {cat }} / \mathrm{K}_{\mathrm{M}} \\
\left(10^{6} \mathrm{M}^{-1} \mathrm{sec}^{-1}\right)\end{array}$ & Reference \\
\hline H. virescens & JHIII & $93-103$ & $2.01^{\#}$ & $2.18^{*}$ & $22 *$ & $\begin{array}{l}\text { Abdel-Aal } \\
\text { et al., } 1988\end{array}$ \\
\hline $\begin{array}{l}H . \text { virescens } \\
\text { (baculovirus } \\
\text { expressed) }\end{array}$ & $\begin{array}{l}\text { JHIII } \\
\mathrm{C}_{6} \mathrm{H}_{13} \mathrm{OCH}_{2}(0) \mathrm{SCH}_{3}\end{array}$ & $\begin{array}{l}30 \\
46000\end{array}$ & $\begin{array}{l}1.57 \\
55.0\end{array}$ & $\begin{array}{l}1.73 \\
60.8\end{array}$ & $\begin{array}{l}58 \\
1.3\end{array}$ & $\begin{array}{l}\text { Ward et al., } \\
1992\end{array}$ \\
\hline H. zea & JHIII & $125-197$ & $4.00^{\#}$ & $4.33^{*}$ & $39 *$ & $\begin{array}{l}\text { Abdel-Aal } \\
\text { et al., } 1988\end{array}$ \\
\hline M. sexta & JHIII & 24 & 1.08 & $1.17 *$ & $49^{*}$ & $\begin{array}{l}\text { Abdel-Aal and } \\
\text { Hammock, } 1985\end{array}$ \\
\hline T. $n i$ & $\begin{array}{l}\text { JHI } \\
\text { JHIII }\end{array}$ & $\begin{array}{l}1130 \\
402 \\
\end{array}$ & $0.058^{\#}$ & $0.046^{*}$ & $0.41 *$ & $\begin{array}{l}\text { Yuhas et al., } \\
1983\end{array}$ \\
\hline $\begin{array}{l}\text { T. } n i \\
(\mathrm{pI}=5.4 / 5.5)\end{array}$ & $\begin{array}{ll}(10 \mathrm{R}, 11 \mathrm{~S}) & \mathrm{JHII} \\
(10 \mathrm{~S}, 11 \mathrm{R}) & \mathrm{JHII}\end{array}$ & $\begin{array}{l}352 / 324 \\
156 / 139\end{array}$ & $\begin{array}{l}2.03 * / 2.12 * \\
0.92 * / 1.11 *\end{array}$ & $\begin{array}{l}2.2 / 2.3 \\
1.0 / 1.2\end{array}$ & $\begin{array}{l}6.3 / 7.1 \\
6.4 / 8.6\end{array}$ & $\begin{array}{l}\text { Hanzlik and } \\
\text { Hammock, } 1987\end{array}$ \\
\hline T. $n i$ & JHII & $\begin{array}{l}380 \\
70.6\end{array}$ & $\begin{array}{l}2.13^{\#} \\
\text { ND }\end{array}$ & $\begin{array}{l}2.34 * \\
0.53\end{array}$ & $\begin{array}{l}6.2 * \\
7.5\end{array}$ & $\begin{array}{l}\text { Rudnicka and } \\
\text { Jones, } 1987 \\
\text { Abdel-Aal and } \\
\text { Hammock, } 1988\end{array}$ \\
\hline L. dispar & $\begin{array}{l}\text { JHIII } \\
(10 \mathrm{R}, 11 \mathrm{~S}) \text { JHII }\end{array}$ & $\begin{array}{l}360^{\mathrm{a}} \\
360^{\mathrm{a}}\end{array}$ & $\begin{array}{l}0.76^{\mathrm{a} \#}\left(2.65^{\mathrm{b \#}}\right) \\
0.48^{\mathrm{a} \#}\end{array}$ & $\begin{array}{l}0.79^{a *} \\
0.49^{a *}\end{array}$ & $\begin{array}{l}2.2^{\mathrm{a} *} \\
1.4^{\mathrm{a} *}\end{array}$ & $\begin{array}{ll}{ }^{\text {a}} \text { Valaitis, } & 1991 ; \\
{ }^{b} \text { Valaitis, } & 1992\end{array}$ \\
\hline G. mellonella & JHI & 21 & $0.92 \#$ & $0.59 *$ & 28 & $\begin{array}{ll}\text { Rudnicka } & \text { and } \\
\text { Kochman, } & 1984\end{array}$ \\
\hline D. melanogaster & $\begin{array}{l}\text { JHIII } \\
\alpha \text {-naphthyl- } \\
\text { acetate }\end{array}$ & $\begin{array}{l}89 \\
120000\end{array}$ & $\begin{array}{l}0.59 \\
70\end{array}$ & $\begin{array}{l}0.60 \\
71\end{array}$ & $\begin{array}{l}6.8 \\
0.59\end{array}$ & This study \\
\hline
\end{tabular}

\#assuming near maximal activity with $5 \mu \mathrm{M} \mathrm{JH}$

*calculated from the reported data assuming that the preparation is fully active

ND not determined 
preferred strongly to the $6 \mathrm{R}, 7$ epoxide, suggesting that $\mathrm{JHB}_{3}$ is an in vivo substrate. However, it may be possible to rationalise these data as follows. The presence of a 6,7 single bond would increase the flexibility of the molecule which might enable the incorrect 10S, 11 epoxide present in the racemic, experimental mixture to more strongly promote binding. In the absence of the (racemic) 10,11 epoxide the natural 6,7 double bond is preferred as expected. Therefore I predict that the 6,7 double bond would have been preferred if 10R-JHIII and its hydrogenated analogues had been available for this experiment.

Binding of JHAs by JHE is promoted by the features found in natural JHIII and $\mathrm{JHB}_{3}$ and reduced by almost any departure from these, suggesting that both molecules are in vivo substrates. However, two JHAs which contain an hydroxyl but lack an epoxide group exhibited unusually high binding affinity for JHE. The location of the hydroxyl groups near the positions of the epoxides of $\mathrm{JHB}_{3}$ suggests either that the hydroxyls can fortuitously substitute for the epoxides in the binding site or that hydroxyls might be found in natural substrates. Incubation of $\mathrm{JHB}_{3}$ with $D$. melanogaster cell fractions gives rise by epoxide hydrolysis to epoxy-diol and tetraol products of $\mathrm{JHB}_{3}$ epoxide hydrolysis together with cis- and trans-tetrahydrofuran diols (Casas et al., 1991). These compounds have the potential to be in vivo substrates for JHE but were not tested in this study. However, as JHIII-diol is not readily bound nor hydrolysed by JHE, the role of hydroxyl-containing substrates remains unclear.

\subsubsection{Interaction of JHE with Lipophorin}

While JHE activity is clearly regulated at the level of synthesis and degradation of JHE (Ichinose et al., 1992a,b), it is possible that its activity is also modulated by interaction with other molecules such as the JH carrier. Two types of interaction with the $\mathrm{JH}$ carrier protein have been proposed (Section 1.5.2). The first type of interaction is a simple mass-action model: Sequestration of $\mathrm{JH}$ by the carrier could be expected to reduce the rate of hydrolysis. However, without the carrier $\mathrm{JH}$ could bind nonspecifically at inaccessible sites in vivo and so might never be available either at its target sites or for degradation. Thus, the carrier might at one time protect $\mathrm{JH}$ from hydrolysis and at another time assist in making it available for hydrolysis.

A second type of interaction occurs in L. maderae in addition to mass-action effects. This interaction requires direct contact between between the carrier, lipophorin, and JHE. In this species lipophorin is also a non-competitive inhibitor of JHE; the rate of $\mathrm{JH}$ hydrolysis being reduced in the presence of lipophorin regardless of the JH concentration (Engelmann, 1984; Engelmann et al., 1988).

L. cuprina lipophorin does not alter the $\mathrm{V}_{\max }$ of $\mathrm{JH}$ hydrolysis by D. melanogaster JHE but it does reduce the rate of JH hydrolysis at lower substrate concentrations. This can be entirely accounted for by a reduction in the free concentration of JH. Thus, only the mass-action model is supported and there is no evidence of direct, inhibitory interaction between lipophorin and JHE in $D$. melanogaster. However, a direct interaction between the D. melanogaster lipophorin and JHE cannot be entirely discounted because L. cuprina, not $D$. melanogaster, lipophorin was used in this study. This could be tested either by purifying the $D$. melanogaster lipophorin or by immunoprecipitation of the $D$. melanogaster lipophorin using antibodies against $L$. cuprina lipophorin which crossreact (Dr. S. Trowell, pers. comm.). 
It is not clear whether all the circulating $\mathrm{JH}$ in D. melanogaster is bound to lipophorin but this carrier has the lowest reported $\mathrm{K}_{\mathrm{D}}$ for JHIII $(1.5 \mathrm{nM})$ of any JH carrier, 59 fold lower than the $\mathrm{K}_{\mathrm{M}}$ of JHE (Shemshedini and Wilson, 1988). The rapid dissociation rate between lipophorin and JHIII suggests that lower quantities of lipophorin might assist JHE and JHEH in the rapid clearance of $\mathrm{JH}$ in vivo but higher quantities could prevent hydrolysis. Knowledge of the developmental regulation of lipophorin in D. melanogaster is therefore necessary for better understanding of its role in $\mathrm{JH}$ titre regulation but this has not been reported.

The mass-action interactions between JHE and the JH carrier may differ between dipterans and lepidopterans because lepidopterans do not use lipophorin as their $\mathrm{JH}$ carrier (Trowell, 1992). The limited available data suggest that the $\mathrm{K}_{\mathrm{D}} \mathrm{s}$ of lipophorin for $\mathrm{JH}$ are much lower than the $\mathrm{K}_{\mathrm{MS}}$ of JHEs in orders which use lipophorin as the $\mathrm{JH}$ carrier (Trowell, 1992). Furthermore, the concentrations of lipophorin appear to exceed JH titres by a considerable margin. Thus, lipophorin may be able to prevent hydrolysis by competition for binding (Trowell, 1992; King and Tobe, 1993). In contrast, Trowell (1992) suggests that the lepidopteran carrier may have limited effectiveness in protecting JH from hydrolysis because the $\mathrm{K}_{\mathrm{D}}$ s of these $\mathrm{JH}$ carriers and the $\mathrm{K}_{\mathrm{MS}}$ of lepidopteran JHEs are similar. However, in T. ni haemolymph, the slow dissociation rate of $\mathrm{JH}$ from the JH carrier limits the rate of JH hydrolysis (Abdel-Aal and Hammock, 1988). In the blattodean, D. punctata a temporally regulated decrease in lipophorin concentration is required for $\mathrm{JH}$ hydrolysis and a decline of $\mathrm{JH}$ titre (King and Tobe, 1993). In contrast, in several other insects, the titre of the carrier rises when JH titre falls and/or JHE activity increases, supporting the proposal that the carrier assists in the clearance of JH (Section 4.3.4). Thus, although the nature of the interactions may differ between species, JH carriers appear to have major roles in the regulation of JH titre. 


\section{Chapter 4}

\section{General Discussion}

\subsection{Introduction}

This study has characterised JH hydrolysis in D. melanogaster and is the first systematic analysis of $\mathrm{JH}$ hydrolysis in any dipteran. Two enzymes have been identified as contributing to $\mathrm{JH}$ hydrolysis, JHE and $\mathrm{JHEH}$, and this chapter discusses the evidence that these enzymes hydrolyse $\mathrm{JH}$ in vivo. The results presented in previous chapters are combined with the available data from the literature to develop a generalised model of the regulation of dipteran $\mathrm{JH}$ titre. The model considers the possible interaction of $\mathrm{JH}$ degradation with the $\mathrm{JH}$ carrier and $\mathrm{JH}$ synthesis, including the possibility of differential regulation of the $\mathrm{JH}$ isoforms, $\mathrm{JHB}_{3}$ and JHIII. Dipteran $\mathrm{JH}$ regulation is compared with regulation in other insect orders, especially the Lepidoptera, to assess the universality of the model. Finally, unresolved issues and future directions for their resolution are discussed.

\subsection{The Specificity of JH Hydrolysing Enzymes and Naphthylacetate Hydrolysis}

\subsubsection{JH-Specificity of D. melanogaster JH Hydrolysing Enzymes}

Several lines of evidence suggest that both JHE and JHEH exhibit a high degree of specificity for $\mathrm{JH}$ as an in vivo substrate rather than functioning as general hydrolytic enzymes. Biological evidence for the JH-specificity of JHE includes maximal expression at the prepupal stage. This is a time when $\mathrm{JH}$ titre is known to fall, $\mathrm{JH}$ synthesis has ceased, interspecific comparisons suggest a requirement for low $\mathrm{JH}$ titre, and $D$. melanogaster are known to be sensitive to exogenous JH. Similarly, biological evidence that $\mathrm{JHEH}$ is $\mathrm{JH}$-specific includes maximal expression during the prewandering period of the final larval instar when "it is becoming increasingly clear that there is an important endocrine event" (Cherbas, 1993), JH synthesis and titre are low, and low $\mathrm{JH}$ titre and increased JH hydrolysis are required in Lepidoptera (Roe and Venkatesh, 1990). The inducibility of JHE by JH in adults suggests that JH-specific $\mathrm{JHE}$ is involved in feedback regulation of JH titre, even though the activity of the enzyme is relatively low at this stage (Ms. S. J. Dent, pers. comm.).

Further evidence of the JH specificity of JHE is provided by its biochemical properties but the biochemical properties of JHEH were not characterised in this study. JHE has a very low $\mathrm{K}_{\mathrm{M}}$ for JHIII and a high specificity constant, $\mathrm{K}_{\mathrm{cat}} / \mathrm{K}_{\mathrm{M}}$, which is greater for JHIII than for the artificial substrate, naphthylacetate. Furthermore, binding experiments demonstrated that JHE exhibits binding selectivity for JHII and $\mathrm{JHB}_{3}$ over other $\mathrm{JH}$ isoforms and analogues, and these are the $\mathrm{JH}$ isoforms most likely to be important in the higher Diptera. 


\subsubsection{The Relationship Between JH Hydrolysis and Naphthylacetate Hydrolysis}

The relationship between JHE and naphthylacetate hydrolysing esterases has been contentious ever since the discovery that $\mathrm{JH}$ induces several naphthylacetate hydrolysing esterases in Hyalophora gloveri pupae which either hydrolyse $\mathrm{JH}$ or comigrate with JHE during native PAGE (Whitmore et al., 1972). It was proposed that "general", naphthylacetate hydrolysing esterases might hydrolyse $\mathrm{JH}$ in vivo in the absence of a protective JH carrier, whereas the specific JHE could also hydrolyse JH bound to the carrier, and that these two classes could be distinguished by DFP inhibition of the general esterases (Sanburg, 1975). There have been several subsequent attempts to identify JHE among esterases separated by native PAGE and visualised with naphthylacetate (East, 1982; Rauschenbach et al., 1987; this study). It is now clear that some purified JHEs cannot hydrolyse naphthylacetate (L. maderae, Gunawan and Engelmann, 1984; G. mellonella (pI = 5.0 variant), Rudnicka and Kochman, 1984, and $M$. sexta, Couldron et al., 1981) whereas others can (T. ni, Hanzlik and Hammock, 1987; G. mellonella ( $\mathrm{pI}=4.75$ variant), Rudnicka and Kochman, 1984), including D. melanogaster (this study).

Naphythylacetate hydrolysis by JHE is never more than a minor component of the total naphythylacetate hydrolysing capacity of insects. However, any compound which contains the ester moiety may have some binding affinity for JHEs, so long as it is not sterically hindered from access to the JHE active site. As naphthylacetate shares no other features with $\mathrm{JH}$ to promote specific binding, high rates of hydrolysis require high substrate concentrations. JHEs from $T$. $n i$ and $H$. virescens have been shown to rapidly hydrolyse other artificial substrates but also require high substrate concentrations (Hanzlik and Hammock, 1987; Ward et al., 1992).

In contrast, it is unlikely that non-specific esterases hydrolyse $\mathrm{JH}$ at low, in vivo concentrations, as the ester group of $\mathrm{JH}$ is both stabilised and unable to rotate, due to conjugation with the 2,3 double bond. Therefore, hydrolysis of $\mathrm{JH}$ requires the enzyme to provide greater activation energy but substrate binding is more sterically constrained. Thus, significant ester hydrolysis of $\mathrm{JH}$ is unlikely except by an enzyme with features that promote specific binding, such as found for D. melanogster JHE. This is supported by the finding that the only zone with JHE activity after native PAGE of D. melanogaster pupae corresponds to the specific JHE, even though greater than physiological concentrations of $\mathrm{JH}$ were used in the assay and several other esterases were present (Section 2.3.2.1). Furthermore EST6, one of the most active D. melanogaster esterases, has no activity with JHIII (Myers, 1990) although it is inducible with JH (Stein et al., 1984; Dr. M. J. Healy and Ms. A. Cao, unpublished data)

\subsection{General Model for JH Regulation in Diptera}

The significance of lepidopteran $\mathrm{JH}$ hydrolysis to the regulation of $\mathrm{JH}$ titre is indicated, in part, by the strong conservation of its temporal and spatial expression patterns among lepidopterans. Therefore, in order to interpret the significance of $\mathrm{JH}$ hydrolysis in $D$. melanogaster, its physiological properties are compared here with data from other dipterans. The data from this study provide a framework to which the more 
scattered data from other dipterans can be fitted to generate an overview of dipteran $\mathrm{JH}$ metabolism. Subsequent comparison of the pattern of expression of dipteran JH metabolism with the available data for $\mathrm{JH}$ synthesis and titre reveals a model for dipteran $\mathrm{JH}$ regulation that is broadly similar to that proposed for other orders.

\subsubsection{General Pattern of Expression of JH hydrolysis in Diptera}

The major observations from several studies of the temporal and subcellular expression patterns of JH hydrolysing enzymes in Diptera are as follows. Adults of $M$. domestica and $D$. melanogaster contain both JHE and JHEH activities, predominantly in the microsomal fraction (Yu and Terriere, 1978a; Ottea et al., 1987) and not in the haemolymph (Wilson and Gilbert, 1978). JHEH in S. bullata and $P$. regina is also predominantly found in the microsomal fraction in adults (Yu and Terriere, 1978b). Most of the JHEH activity of larvae of D. melanogaster (Casas et al., 1991; Harshman et al., 1991) and D. hydei (Klages and Emmerich, 1979) appears to be mitochondrial and microsomal, supporting data from other insects that JHEH is generally membranebound (Roe and Venkatesh, 1990). No JH degrading enzymes are found in the haemolymph of $D$. melanogaster or $D$. hydei final instar larvae before pupariation (Wilson and Gilbert, 1978; Klages and Emmerich, 1979; Shemshedini and Wilson, 1988). However, JH degrading enzymes are found in the fat body and body wall of $D$. hydei larvae. A maximum of JHE activity occurs in the prepupal haemolymph of $D$. hydei four hours after the formation of the puparium but activity then declines gradually through the entire pupal stage (Klages and Emmerich, 1979). The above data are consistent with the finding that microsomes of $M$. domestica, S. bullata and $P$. regina larvae and adults contain higher activities of $\mathrm{JH}$ degrading enzymes than are present in pupal microsomes, however non-microsomal activities were only determined for adults ( $\mathrm{Yu}$ and Terriere, 1978a,b). In M. domestica larvae, a prewandering peak of microsomal JHEH activity occurs (Yu and Terriere, 1978b). In D. virilis low JHE activity is found in final instar larvae but a maximum for JHE is found in pupae. In contrast, JHEH activity is highest earlier in final instar larvae. Thus, a minimum for JH hydrolysis occurs just prior to pupariation (Rauschenbach et al., 1991). To these observations can be added the results of my study that prewandering and prepupal maxima of $\mathrm{JH}$ hydrolysis occur in $D$. melanogaster, and that $\mathrm{JH}$ degrading activities in larvae and adults are predominantly mitochondrial and microsomal, whereas the pupal activity is predominantly soluble JHE.

Taken together, these data suggest the following generalisations about the pattern of expression of JHE and JHEH in Diptera. In final instar larvae, JH hydrolysing enzymes are found in tissues other than the haemolymph and activity is greatest during the prewandering period. The relative contributions of JHE and JHEH to total activity vary among species but JHEH is always important. There is a minimum of activity late in the final instar, but a high level of soluble, circulating JHE activity occurs soon after the formation of the puparium and before the pupal moult, probably clearing $\mathrm{JH}$ from the whole organism. This JHE is retained, though declining in activity, through the pupal stage. In the adults low levels of both JHE and JHEH are found, though with different subcellular distribution. 


\subsubsection{Correlation of $\mathrm{JH}$ Hydrolysis with $\mathrm{JH}$ Production and Titre Changes}

The developmental profile of $\mathrm{JH}$ hydrolysis correlates inversely with $\mathrm{JH}$ titre and production in D. melanogaster (section 2.4.2). Less complete data suggest that $D$. hydei exhibits a very similar inverse correlation of $\mathrm{JH}$ hydrolysis (Klages and Emmerich, 1979) with JH titre (Bührlen et al., 1984) but JH production has not been investigated in $D$. hydei. JHIII was the only $\mathrm{JH}$ isoform detected when the developmental profiles of JH titres in $D$. melanogaster and $D$. hydei were determined. However, $\mathrm{JHB}_{3}$ was neither proposed to be a hormone nor tested for at the time of these studies. Developmental changes in JH hydrolysis in D. melanogaster correlate well with critical endocrinological events. The relationships between changes of JH hydrolysis, production and titre and other processes during important periods in dipteran development are discussed below (and illustrated in Figure 2.8).

\subsubsection{The Prewandering Period of the Final Larval Instar}

In $D$. melanogaster at the prewandering stage a small peak of ecdysone occurs (Cherbas, 1993), JH titre is reduced (Bownes and Rembold, 1987; Sliter et al., 1987), and this study now shows that $\mathrm{JH}$ hydrolysis is elevated. D. hydei also exhibits a reduction of $\mathrm{JH}$ titre just prior to the onset of wandering (Bührlen et al., 1984). Although this has not been related to changes in rates of JH hydrolysis, JHE and JHEH occur in D. hydei tissues at this time (Klages and Emmerich, 1979) and a prewandering peak of microsomal JHEH occurs in $M$. domestica (Yu and Terriere, 1978b). In lepidopterans prewandering JHE reduces $\mathrm{JH}$ titre and leads to a prewandering ecdysone peak (Jones, 1985; Watson et al., 1987).

These changes in endocrine status are associated with numerous changes in behaviour and gene expression in both dipterans and lepidopterans (Cherbas, 1993). Thus, the function of the prewandering ecdysone peak together with low $\mathrm{JH}$ titre appears to be induction of the first events in the onset of metamorphosis (Andres and Thummel, 1992; Shaaya, 1993). At the molecular level one of the first responses in insect epidermal cells is a brief cessation of RNA synthesis followed by the production of a novel RNA type (hetero-disperse nuclear RNA, hnRNA) (Shaaya, 1993). The hnRNA may have a role in the permanent repression of larval mRNA synthesis and/or the induction of pupal genes. The changes in RNA synthesis are observed in the lepidopterans, M. sexta and Ephestia cautella, and the dipteran Calliphora vicina. The importance of the low $\mathrm{JH}$ titre is indicated by application of the $\mathrm{JH}$ analogue, methoprene, which, if applied before the appearance of hnRNA, prevents these changes in RNA synthesis and delays the larval-pupal transformation .

\subsubsection{Prepupae/Pupae}

During the late larval/prepupal period two further ecdysone peaks occur in D. melanogaster. The first of these occurs just prior to puparium formation (Andres and Thummel, 1992) and coincides with high JH titre (Sliter et al., 1987) and JH production (Richard et al., 1989a). This study now shows that a minimum of JH degradation also occurs at this time. The second ecdysone peak occurs 10 hours later (Andres and Thummel, 1992) and coincides with a low JH titre (Bownes and Rembold, 1987) and the pupal apolysis (Bainbridge and Bownes, 1981). It also follows a cessation of JH synthesis (Richard et al., 1989a) and this study now shows that a 
maximum of $\mathrm{JH}$ degradation occurs at this time. An equivalent pattern of ecdysteroid peaks is also observed in the dipteran, Sarcophaga crassipalpis (Zdárek and Denlinger, 1987). In $D$. hydei, a peak of JH occurs after the onset of wandering. Late in the final instar JH titre falls ahead of the prepupal peak of haemolymph JHE activity which does not appear until shortly after pupariation and $\mathrm{JH}$ is barely detectable during the prepupal stage (Klages and Emmerich, 1979; Bührlen et al., 1984).

These events in Diptera all correspond well with similar events in the Lepidoptera (Baker et al., 1987): lepidopteran pupation is initiated by the coincidence of a pulse of ecdysone and a pulse of $\mathrm{JH}$ production and $\mathrm{JH}$ titre then falls to low levels ahead of the prepupal peak of JHE. The JH titre falls to essentially zero when the prepupal JHE appears, and this is followed by pupal apolysis.

The prepupal pulse of $\mathrm{JH}$ is believed to prevent adult differentiation of the imaginal discs during the pupal moult (Kiguchi and Riddiford, 1978). In both lepidopterans and dipterans, JH titres fall to low levels ahead of high levels of JHE. Thus, it appears that the function of elevated prepupal JHE activity is to scavenge for trace levels of $\mathrm{JH}$ after most has been removed by constitutive levels of the enzyme or other means (Baker et al., 1987). Inhibition of the lepidopteran prepupal JHE peak causes larval-pupal intermediates and blocks ecdysis (Newitt and Hammock, 1986). In D. virilis the organophosphate esterase inhibitor paraoxon causes pupal death when applied to prepupae at concentrations which do not inhibit the majority of naphthylacetate hydrolysing esterases (Rauschenbach et al., 1991). As paraoxon is a potent inhibitor of JHE in several dipterans (Yu and Terriere, 1978b; Klages and Emmerich, 1979) this effect may be due to inhibition of JHE.

The effects of inhibitors of $\mathrm{JH}$ hydrolysis have not been investigated in vivo in D. melanogaster. The identification of selective inhibitors of JHE and their use at this time in D. melanogaster may identify specific metamorphic changes which require JHE activity. These may include the secretion of the pupal abdominal cuticle and subsequent replacement of larval epidermal cells by abdominal histoblasts as this process is disrupted by JH application (Section 1.4.1).

During the pupal stage of $D$. melanogaster a further ecdysone peak occurs (Riddiford, in press) coinciding with pupal to adult apolysis (Bainbridge and Bownes, 1981). Pupal JH titre is low (Bownes and Rembold, 1987; Sliter et al., 1987) and this study shows that $\mathrm{JH}$ hydrolysis is elevated though declining throughout this period. Similarly, JHE activity remains elevated though declining throughout the pupal stage of D. hydei and $\mathrm{JH}$ remains barely detectable (Klages and Emmerich, 1979; Bührlen et al., 1984). Application of JH to pupae of the dipteran, S. bullata causes the secretion of a second cuticle with pupal characteristics (Srivastava and Gilbert, 1968; 1969). Thus, in common with Lepidoptera and other insects, the change from pupal to adult differentiation requires the absence of JH (Willis, 1981; Willis and Cox, 1984).

\subsubsection{Adults}

In $D$. melanogaster, JHIII titre rises rapidly after adult eclosion and remains high (Bownes and Rembold, 1987). Maximum $\mathrm{JHB}_{3}$ production occurs in adult female D. melanogaster at the same time as other indicators of sexual maturity on day 2 (Altaratz et al., 1991) and JH hydrolytic activity remains low during the first five days of the adult stage. Thus, an elevated JH titre correlates with elevated JH synthesis and low hydrolytic activity. However, mated and virgin Drosophila have not been compared for $\mathrm{JH}$ titre or $\mathrm{JH}$ hydrolysis. $\mathrm{JH}$ hydrolysis and production have also not 
been investigated in adult $D$. hydei although $\mathrm{JH}$ titre rises less rapidly in female $D$. hydei than $D$. melanogaster, and less rapidly still in male $D$. hydei, reflecting their slower rates of sexual maturation (Bührlen et al., 1984).

Although JHE and JHEH activities are lower in adult D. melanogaster than at other stages, JHE can be induced about $30 \%$ in females and $60 \%$ in males by topical application of JHIII (Ms. S. J. Dent, pers. comm.). Furthermore, JH synthesis appears to be required for stimulation of yolk protein uptake by oocytes (Saunders et al., 1990), suggesting that regulation of $\mathrm{JH}$ titre by both $\mathrm{JH}$ synthesis and hydrolysis remains important in adults. Thus, JH synthesis and hydrolysis may vary in response to mating or other conditions which have not yet been tested.

In contrast to prepupae, more than half of the JHE as well as the JHEH activity is found in membranous cell fractions of whole adults (Section 2.3.2.3), suggesting that hydrolysis might mainly occur in specific tissues rather than throughout the organism. Thus, tissues may independently modulate the $\mathrm{JH}$ signal by hydrolysis or turn off the $\mathrm{JH}$ signal after it has reached its site of action.

JHE has a clear role in the regulation of JH during egg production in adult females of the dipteran A. aegypti (Shapiro et al., 1986; Borovsky et al., 1992) At the start and finish of a cycle of egg production JH titre is high and JHE activity is low, whereas the opposite occurs in the middle of the cycle. Inhibition of JHE causes an increase in $\mathrm{JH}$ titre and a reduction in egg hatch. Similar relationships between egg production, JH titre and JH hydrolysis have been observed in an orthopteran (Renucci et al., 1984), a blattodean (Tobe et al., 1985), and appear likely in a lepidopteran (Venkatesh et al., 1988).

\subsubsection{Further Analysis of JH Titre Regulation}

In order to fully understand the role of hydrolysis in the regulation of JH titre, further investigation in several areas in required. The investigation of the pattern of expression of $\mathrm{JH}$ hydrolysing enzymes should be extended to include earlier larval stages, tissue localisation and the response of enzyme activities to mating. Determination of the effects of inhibition of JHE and JHEH in vivo would also contribute to understanding of the roles of these enzymes. However, care would be required for the interpretation of such experiments because other esterases or epoxide hydrolases may be inhibited. Paraoxon and OTFP (among others) have been used to inhibit JHEs in vivo but both inhibit numerous other D. melanogaster esterases (Healy et al., 1991). To complement such data more detailed titres of both JHIII and $\mathrm{JHB}_{3}$ throughout development are required.

\subsubsection{Compartmentalisation and Relative Contributions to JH Hydrolysis of JHE and JHEH}

The relative contributions of JHE and JHEH to prewandering activity vary among dipteran species although JHEH is always important. Comparison with $D$. hyde $i$ and Lepidoptera suggests that prewandering dipteran $\mathrm{JH}$ hydrolysing enzymes will be found in a wide range of tissues, In contrast it is probable that a large portion of the dipteran prepupal JHE will be found the haemolymph because $D$. melanogaster JHE is soluble and in $D$. hydei and Lepidoptera it is found in the haemolymph. Comparison of the dipteran and lepidopteran developmental profiles of hydrolysis, synthesis, and titre suggest that the function of hydrolysis by both JHEH and JHE at both these times 
is to clear JH from the whole organism (Section 4.3.2). In adult dipterans, low levels of both JHE and JHEH are found, though with different subcellular distribution.

While most studies of $\mathrm{JH}$ hydrolysis in insects have concentrated on haemolymph, a few studies of lepidopteran larvae have investigated hydrolysis in other tissues. These studies indicate that JHE and JHEH activities in tissues exhibit prewandering and prepupal peaks, suggesting that non-haemolymph JH hydrolysis may be as important as haemolymph hydrolysis (Section 1.6.2). Thus, the most obvious difference between JH hydrolysis in dipterans and lepidopterans is the lack of JHE in the haemolymph of prewandering dipterans.

The lack of JHE in the haemolymph of prewandering dipterans may not be unusual. The coleopteran, Tenebrio molitor, has a haemolymph JHE peak which coincides with pupal ecdysis and a JH sensitive period. Thus, its prepupal pattern resembles both Diptera and Lepidoptera (Connat, 1983). However, in common with the Diptera and unlike the Lepidoptera, no othe- haemolymph JHE peak occurs during the final larval instar. Whole larvae of another coleopteran, Tribolium castaneum, exhibit high levels of non-haemolymph JHEH activity (Connat, 1983), suggesting that the pattern of expression of $\mathrm{JH}$ hydrolytic enzymes in Coleoptera may more closely resemble the dipteran model than the lepidopteran model. While JHEH activities have not been studied in the same detail as JHE, JHEH occurs in whole organisms in conjunction with JHE at a variety of developmental stages in many insect orders (Slade and Zibitt, 1972; Ajami and Riddiford, 1973). Thus, it is not clear why prewandering dipterans and coleopterans lack haemolymph hydrolysis, or alternatively, why the Lepidoptera need it. It is also unknown whether JHE and JHEH differ in their functions.

JHE may be absent from general circulation at the prewandering stage of some insects including Diptera because the elimination of JH is only required by some tissues. For example, JH hydrolysis in imaginal discs from final instar D. melanogaster larvae appears to be sufficient to render these tissues insensitive to circulating $\mathrm{JH}$, with much greater than physiological concentrations of $\mathrm{JH}$ required in vitro for inhibition of ecdysone-induced evagination (Chihara et al., 1972). Alternatively, JH hydrolysis within restricted tissues may be sufficient to eliminate $\mathrm{JH}$ throughout the organism. The latter has not been shown in dipteran larvae but non-haemolymph metabolism appears to be sufficient for $\mathrm{JH}$ clearance from whole adult $M$. domestica; in a comparative study across six insect orders the haemolymph JHE activity of adult $M$. domestica was the lowest found, yet the half life of injected JHI was lowest in this species (de Kort et al., 1979).

There is considerable variation in the relative titres of JHE and JHEH both within and between insect orders. However, there does appear to be a trend for greater hydrolysis by JHEH at the prewandering stage of the final instar and greater hydrolysis by JHE at the prepupal stage (Section 1.6.1). The significance of this observation is far from clear although there are several hypotheses. Firstly, different routes of hydrolysis may influence the ultimate fate of $\mathrm{JH}$. Hydrolysis by $\mathrm{JHEH}$ causes absolute loss of $\mathrm{JH}$ from circulation because there is no known mechanism for reformation of the epoxide group from $\mathrm{JH}$-diol. In contrast, $\mathrm{JH}$-acid can be methylated by $\mathrm{JH}$ methyltransferase to reform JH in some tissues (Sparagana et al., 1985). Thus, in the presence of JH-acid, some tissues may be able to maintain a locally elevated $\mathrm{JH}$ titre in spite of generally reduced titres, or influence other tissues by release of remethylated JH. Tissues with 
JH methyl transferase activity include imaginal discs, suggesting a mechanism by which developing discs might influence the timing of metamorphosis.

In addition, differences between substrate specificities of JHE and JHEH may permit the selective hydrolysis of different $\mathrm{JH}$ isoforms. To date, there is evidence for more than one $\mathrm{JH}$ isoform in the Lepidoptera and the Diptera but no functional differences between $\mathrm{JH}$ isoforms have been demonstrated. In $D$. melanogaster larvae the relative rates of JHEH hydrolysis of $\mathrm{JHB}_{3}$ and JHIII differ between subcellular fractions, suggesting that there is more than one $\mathrm{JHEH}$ variant and that the two JH isoforms might be hydrolysed at different rates at different sites in vivo.

Investigation of the tissue localisation of JHE and JHEH by dissection and assay of individual organs is an important future direction for research. However, high resolution analysis would require antibodies raised against purified enzymes. D. melanogaster JHEH has not been purified but the JHE purification protocol developed for the current study would enable the generation of JHE antibodies. Such approaches could be complemented with in situ RNA hybridisation studies once the genes for JHE and JHEH are isolated.

Determination of the tissue localisation of JHE and JHEH at times of minimum $\mathrm{JH}$ hydrolytic capacity is important to assess the relationship between local titres of $\mathrm{JH}$ within tissues and the circulating titres. It may be that the enzymes will be found at high concentration in restricted tissues which need to maintain low local JH concentrations in spite of high circulating titres. Kinetic characterisation of purified JHEHs would also be an important complement to the current study.

\subsubsection{The Role of Lipophorin in JH Titre Regulation}

This study has demonstrated that changing the relative concentrations of dipteran JHE and lipophorin modulates the rate of in vitro JH hydrolysis by JHE but it is not known whether lipophorin modulates $\mathrm{JH}$ hydrolysis in vivo. The in vivo concentration and JHIII binding kinetics of lipophorin from haemolymph of D. melanogaster wandering larvae have been determined but it is not clear whether this lipophorin titre is sufficient to bind all the JH because of uncertainty about the JHIII titre (Shemshedini and Wilson, 1988). Furthermore, the titre and binding kinetics of $\mathrm{JHB}_{3}$ with lipophorin are unknown and it is also unknown whether lipophorin is developmentally regulated in D. melanogaster.

However, studies of the high affinity $\mathrm{JH}$ carrier protein of $A$. aegypti (presumably lipophorin) suggest that it has a role in the regulation of JH titre. A sixfold increase in JH carrier protein occurs within one hour of a blood meal, coinciding with a JH titre decline and a JHE peak which are required for egg production (Shapiro et al., 1986; Thomas et al., 1986 cited in Borovsky et al., 1992). However, in $M$. domestica, lipophorin exhibits only slight titre variation through development (de Bianchi et al., 1987).

The titres of JHE and the JH carrier also rise and fall in concert in haemolymph of the lepidopteran, G. mellonella, and the blattodean, L. maderae, (Ozyhar et al., 1983; Engelmann et al., 1988). In these examples the increase in carrier titre is proposed to assist in the clearance of JH. Therefore, interaction between JH carriers and hydrolytic enzymes appears to be important in at least three insect orders. However, the nature of the interaction differs between blattodeans. In contrast to L. maderae, a specific decline of lipophorin titre in D. punctata is proposed to be critical for clearance of JH from the 
haemolymph (King and Tobe, 1993). The decline, which occurs just prior to oviposition, coincides with elevated haemolymph JHE and reduced JH titre. The titres of $\mathrm{JH}$ and lipophorin, the $\mathrm{K}_{\mathrm{M}}$ of $\mathrm{JHE}$, and the $\mathrm{K}_{\mathrm{D}}$ of lipophorin for $\mathrm{JH}$, lead to the conclusion that virtually all the $\mathrm{JH}$ would be bound and $\mathrm{JH}$ hydrolysis prevented in vivo if the decline in lipophorin titre did not occur. These contrasting examples demonstrate that careful consideration of all the kinetic parameters describing the behaviour of both the hydrolytic enzymes and the carrier, together with accurate titres of all components of the system, will be required for prediction of interactions. It should be noted also that developmental changes of lipophorin titre may occur due to changing requirements for another of its functions such as lipid transport (Trowell, 1992).

Whether circulating lipophorin affords protection to $\mathrm{JH}$ from intracellular enzymes depends in part on how readily $\mathrm{JH}$ moves between compartments. JH synthesised by the corpus allatum in vitro is protected from degradation by enzymes within the organ by lipophorin added to the incubation medium, suggesting that mass action effects for binding can act across tissue boundaries (Lanzrein et al., 1993). However, it remains to be determined whether haemolymph $\mathrm{JH}$ carriers can afford protection from enzymes in other tissues.

\subsection{Roles and Regulation of JH Isoforms}

\subsubsection{Identification and Functions of JH Isoforms}

The identification of the functional JH isoform(s) of the higher Diptera remains inconclusive (Section 1.2), although as the major biosynthetic product, $\mathrm{JHB}_{3}$ is probably functional. However, the role of JHIII is not yet defined. JHIII may be merely a precursor of $\mathrm{JHB}_{3}$ that has no other function, or it may have a role which is more or less distinct from that of $\mathrm{JHB}_{3}$.

Bioassays in D. melanogaster provide examples of both greater sensitivity to JHIII over $\mathrm{JHB}_{3}$ and vice versa, suggesting that the two $\mathrm{JH}$ isoforms have different functions. $\mathrm{JHB}_{3}$ has more potent effects on ovarian maturation than JHIII and it may be significant that the synthesis of $\mathrm{JHB}_{3}$, but not JHIII, is developmentally regulated in adult females (Saunders et al., 1990; Altaratz et al., 1991). In contrast, JHIII is about ten-fold more potent than $\mathrm{JHB}_{3}$ for production of various adult defects when topically applied to white prepupae and synthesis of both $\mathrm{JHIII}$ and $\mathrm{JHB}_{3}$ is developmentally regulated at this stage (Richard et al., 1989a,b). This might suggest that the role of JHIII is restricted to metamorphosis whereas $\mathrm{JHB}_{3}$ has both a metamorphic and reproductive role. Such results should be treated with caution, however, because nonphysiological $\mathrm{JH}$ isoforms and analogues are sometimes more potent, possibly due to less rapid degradation (Schwieter-Peyer, 1973; Wyatt et al., 1987; Richard et al., 1989 b). The binding specificity of $D$. melanogaster JHE revealed in my study supports a function for both isoforms because JHIII and the natural optical isomer of $\mathrm{JHB}_{3}$ are both bound with high affinity by JHE.

More than one $\mathrm{JH}$ isoform occur in at least two holometabolous insect orders (Diptera, if JHIII is functionally important in vivo, and Lepidoptera) and one hemimetabolous insect order (Hemiptera, Numata et al., 1992). The appearance of more than one $\mathrm{JH}$ isoform in an insect may reflect a need for more complex regulation of physiological events in some orders (Bollenbacher, 1988; Cusson et al., 1991). It 
may be possible to infer any differences in the roles of the $\mathrm{JH}$ isoforms by observing temporal or spatial correlations between isoform-specific degradation, isoform titres, physiological events and isoform sensitivity in different bioassays. It is noteworthy that differential regulation of two ecdysteroids occurs in the lepidopteran, Orgyia postica, suggesting that ecdysteroid isoforms may also have different functions through development and between the sexes. However, no such functions have been defined (Gu et al., 1992).

Although no distinct roles for JH isoforms have been demonstrated, differences in carrier binding, rates of production and rates of degradation suggest that $\mathrm{JH}$ isoforms are differentially regulated in both Diptera and Lepidoptera. These issues are discussed below.

\subsubsection{Differential Regulation of JH Isoforms}

\subsubsection{Synthesis of JH Isoforms}

Although developmental profiles for dipteran $\mathrm{JHB}_{3}$ titre have not been determined, the available evidence suggests that differential regulation of JHIII and $\mathrm{JHB}_{3}$ could occur at the level of synthesis and/or degradation. In final instar larvae of D. melanogaster production of $\mathrm{JHB}_{3}$ and $\mathrm{JHIII}$ rise and fall in concert but in adult females the production of JHIII remains largely unchanged as the production of $\mathrm{JHB}_{3}$ changes (Richard et al., 1989a; Altaratz et al., 1991). The presumed precursor of $\mathrm{JHB}_{3}$, farnesoic acid, has no effect on in vitro $\mathrm{JHB}_{3}$ production by corpora allata of adult $C$. vomitoria but does lead to an increase in JHIII production, suggesting that a 6,7 epoxidase could be regulated to determine whether the product of the pathway is JHIII or $\mathrm{JHB}_{3}$ (Duve et al., 1992).

Among the Lepidoptera the profile of JH isoform synthesis changes during development. For example, in $M$. sexta JH production shifts from exclusively 4-MeJHI and $\mathrm{JHO}$ in embryos, to predominantly JHI and JHII in larvae, to JHII and JHIII in adults (Schooley and Baker, 1985). Also, JHII titre exceeds JHI at the start of the final instar but the reverse occurs for rest of the instar (Baker et al., 1987).

\subsubsection{Degradation of JH Isoforms}

Selective degradation of $\mathrm{JH}$ isoforms can cause the actual ratio of $\mathrm{JH}$ isoforms to differ from the synthesised ratio (Szolajska, 1991). The relative abundance of JH isoforms could be developmentally regulated if multiple JH degrading enzymes with different isoform specificity are expressed at different times. Similarly, the relative abundance of $\mathrm{JH}$ isoforms could be spatially regulated by spatially regulated $\mathrm{JH}$ degrading enzymes. Isoform-specific degradation can be further modulated by selective protection of JH isoform(s) by the JH carrier (Szolajska, 1991).

The present study shows that the predominant route and site of JH hydrolysis changes through development, indicating the potential for developmental and spatial regulation of isoform selective degradation. Furthermore, the available evidence suggests that more than one JHEH variant occurs in larval tissues, that these differ in their specificities for $\mathrm{JHIII}$ and $\mathrm{JHB}_{3}$, but that JHIII is generally the preferred substrate (Casas et al., 1991; Harshman et al., 1991). Prepupal JHE has a higher binding affinity for JHIII over $\mathrm{JHB}_{3}$ which may also indicate selectivity for JHIII (Section 3.4.3.2), but there may be a different JHE variant in adult D. melanogaster (Section 2.4.3), and the maximum rate of hydrolysis of $\mathrm{JHB}_{3}$ by JHE remains to be tested. JHEs from several 
lepidopteran species also exhibit variation of $\mathrm{K}_{\mathrm{M}}$ and/or specificity constants for different $\mathrm{JH}$ lepidopteran isoforms (Table 3.2), which may contribute to isoform specific regulation.

\subsubsection{Interactions Among Carrier Binding, Synthesis and Degradation of JH Isoforms}

Developmental and spatial regulation of particular JH isoforms may be achieved by a balance of synthesis and degradation, modulated by carrier binding, as is proposed for general JH regulation. L. cuprina and D. melanogaster lipophorins both have high affinities for JHIII, with preliminary results suggesting that $L$. cuprina lipophorin has a lower affinity for $\mathrm{JHB}_{3}$ (Shemshedini and Wilson, 1988; Dr. S. C. Trowell, pers. comm.). Thus, abundant lipophorin might protect JHIII from hydrolysis but leave $\mathrm{JHB}_{3}$ largely unbound and available for hydrolysis. At another time a lower lipophorin titre might not afford protection to either $\mathrm{JHIII}$ or $\mathrm{JHB}_{3}$ (and may promote transport to the site of degradation). Hydrolytic enzymes might then hydrolyse their preferred substrate, JHIII. Thus, interaction between lipophorin and JH hydrolysing enzymes could cause the actual ratio of JHIII to $\mathrm{JHB}_{3}$ to differ from the synthesized ratio.

In an analysis of the kinetics of binding of JH to JHE and the JH carrier in $T$. ni it was concluded that "even low levels of JHE could shift the relative titres of JH homologues observed if their relative equilibrium and kinetic dissociation constants with the JH binding protein differed" (Abdel-Aal and Hammock, 1988). Supporting the hypothesis that interaction between the carrier and hydrolytic enzymes can affect the relative abundance of natural $\mathrm{JH}$ isoforms is the observation that interaction between carriers and JHEs can result in preferential hydrolysis of one optical isomer of JH from experimental racemic mixes. Whether the physiological or non-physiological isomer is hydrolysed depends on whether the carrier or the esterase has the greater selectivity between isomers (Peter et al., 1979; de Kort et al., 1983; Schooley et al., 1984; Meyer and Lanzrein, 1989).

Final instar larvae of the lepidopteran, G. mellonella, provide the most complete example of interaction between $\mathrm{JH}$ production, carrier binding and hydrolysis for the regulation of $\mathrm{JH}$ isoforms. Haemolymph titres of the $\mathrm{JH}$ carrier and $\mathrm{JHE}$ increase and decrease in concert (Ozyhar et al., 1983), so the carrier has been proposed to assist in the clearance of JH from insect's tissues (Wing et al., 1984; Trowell, 1992). However, three isoforms of JH (I, II, and III) are synthesised. Hydrolytic rates in the haemolymph and corpus cardiacum-corpus allatum complex are maximal with JHI and JHIII respectively, and JHII is most resistant to hydrolysis in both locations. JHII binding by the haemolymph carrier is slightly more stable than for JHI and about seven times more than for JHIII. Thus, preferential binding of JHII and hydrolysis of the other isoforms leads to JHII being the dominant form (Szolajska, 1991). The whole body content of JHII-acid follows the JHII titre indicating that only a portion of JHII is hydrolysed even with peak JHE activities. In contrast, high JHIII-acid titres are associated with the near total absence of JHII, indicating that hydrolysis can effectively eliminate JHIII.

\subsubsection{Future Directions for the Analysis of JH Isoform Roles and Regulation}

A complete analysis of the regulation of $\mathrm{JHIII}$ and $\mathrm{JHB}_{3}$ in $D$. melanogaster may contribute to the assessment of the function of each isoform. Important experiments yet to be performed include determination of the developmental profile of 
the $\mathrm{JHB}_{3}$ titre, the kinetics of $\mathrm{JHB}_{3}$ binding by lipophorin, and the spatial expression pattern of JHE and JHEH.

An important experiment to complement the characterisation of JHII hydrolysis by JHE (Section 3.4.3) is characterisation of hydrolysis of $6 \mathrm{~S}, 10 \mathrm{R}-\mathrm{JHB}_{3}$. While the $\mathrm{K}_{\mathrm{M}}$ of JHE for JHIII is greater than the $\mathrm{K}_{\mathrm{I}}$ for $\mathrm{JHB}_{3}$, it is unknown how the $\mathrm{K}_{\text {cat }}$ compares between these two substrates. For $\mathrm{JH}$ concentrations which are much less than the $\mathrm{K}_{\mathrm{M}}$ (such as occur in vivo) it is the specificity constant $\left(\mathrm{K}_{\mathrm{cat}} / \mathrm{K}_{\mathrm{M}}\right)$ which determines the reaction rate (Abdel-Aal and Hammock, 1988). If the $\mathrm{K}_{\mathrm{M}}$ and $\mathrm{K}_{\text {cat }}$ for $\mathrm{JHB}_{3}$ are both higher than for JHIII, the specificity constant may be similar for the two isoforms. This would enable JHE to hydrolyse $\mathrm{JHB}_{3}$ as efficiently as JHIII. If both JHIII and $\mathrm{JHB}_{3}$ are in vivo substrates, a higher $\mathrm{K}_{\mathrm{M}}$ for $\mathrm{JHB}_{3}$ but similar specificity constant might reflect higher physiological titres of $\mathrm{JHB}_{3}$.

Sufficient quantities of $6 \mathrm{~S}, 10 \mathrm{R}-\mathrm{JHB}_{3}$ to perform these experiments can now be separated from chemically synthesised, racemic $\mathrm{JHB}_{3}$ (Hearlt et al., 1993) and could be radiolabelled in the carboxyl chain. Alternatively, $6 \mathrm{~S}, 10 \mathrm{R}-\mathrm{JHB}_{3}$ radio-labelled in the methyl-alcohol moiety can be biosynthesised in vitro using $L$. cuprina corpora allata (Dr. P. East, pers. comm.). The JHE assay procedure for methyl-radio-labelled JH is essentially the same as used in present study (Hammock and Roe, 1985). Therefore, direct measurement of $6 \mathrm{~S}, 10 \mathrm{R}-\mathrm{JHB}_{3}$ hydrolysis by JHE is now possible.

\subsection{Structure and Regulation of JHE}

This study has analysed aspects of the regulation of JH by JHE at the protein level. There are now issues arising from this study which may best be addressed at the nucleic acid level, requiring isolation of the gene encoding JHE. This study has identified $\mathrm{JH}$ hydrolysis as an important factor in the regulation of $\mathrm{JH}$ titre. For deeper understanding of the regulation of $\mathrm{JH}$ titre it is now necessary to identify the factors involved in the temporal and spatial regulation of $\mathrm{JH}$ hydrolysis. One such factor is $\mathrm{JH}$ itself which may self-regulate by feedback induction of JHE. Other potential regulatory factors include ecdysone, "head factors" and tissue-specific transcription factors (Section 1.6.3).

Several approaches to the isolation of the D. melanogaster JHE gene have been pursued (Ms. S. J. Dent, Ms. M. M. Dumancic and Dr. M. J. Healy, unpublished data). However, the cDNA of $H$. virescens JHE (Hanzlik et al., 1989) does not hybridise with $D$. melanogaster genomic DNA and antibodies raised against lepidopteran JHEs do not cross-react with $D$. melanogaster JHE, indicating that $D$. melanogaster and lepidopteran JHE are dissimilar. The gene may be obtained by probing DNA libraries with the oligonucleotides corresponding to the $\mathrm{N}$-terminal amino acid sequence of the JHE purified in this study but the seven amino acid $\mathrm{N}$-terminal sequence (Section 3.3.2.4) does not appear to be sufficient for unique identification of the JHE gene. However, more JHE could to be purified to obtain either a longer Nterminal sequence or internal amino acid sequence data after cleavage and separation of peptides. The peptide containing the presumed active site serine could be identified by treatment of JHE with radioactive DFP prior to cleavage, as has been achieved with other esterases (Ozols, 1987). Internal sequence data would enable the use of techniques including the polymerase chain reaction to obtain the DNA sequence between the $\mathrm{N}$-terminus and the peptide containing the active site serine. 
Isolation of the JHE gene would enable the identification of amino acid residues required for catalytic activity and substrate specificity, and RNA hybridisation would enable the site(s) of synthesis of JHE to be determined with high precision. Sequence comparison between the $H$. virescens and $D$. melanogaster JHE genes should enable the identification of conserved structural features of the protein. These are likely to include the residues of the catalytic triad and residues involved in maintainance of the secondary and tertiary structure of members of the esterase/lipase family (Section 1.8) but might also include shared features around the active site which confer $\mathrm{JH}$ specificity. The sequences of many esterases are known but unequivocal physiological roles are only known for JHE and AChE. Site directed mutagenesis experiments among the apparently dissimilar lepidopteran and dipteran JHEs and AChE could further help to identify features required for $\mathrm{JH}$ binding and others required for esterase function in general (Ward et al., 1992).

It is probable that the regulation of JHE in D. melanogaster will prove as complex as it is in lepidopterans (Section 1.6). The results from this study, together with data from the literature, suggest that JHE is regulated in response to both ecdysone and JH because its activity rises from its minimum value to its maximum value between two pulses of ecdysone and shortly after a pulse of JH. Full characterisation of the regulation JHE may therefore provide insights into the interactions between these two hormones for the correct regulation of gene expression through the larval to pupal transition. Comparison between the $H$. virescens and $D$. melanogaster JHE genes may enable the identification of tissue-specific or hormone responsive elements, although these may diverged considerably between these two species. Another comparison which may prove fruitful is between the genes for JHE and EST6. Both genes are induced by $\mathrm{JH}$ in adult $D$. melanogaster and the gene for EST6 is well characterised (Ludwig et al., 1993; Healy et al., in preparation), but the JH responsive element(s) remain to be identified. Identification of such elements would assist in the identification and isolation of other $\mathrm{JH}$ regulated genes and in the construction of a model for the mechanism of JH action on gene expression. The availability of transformation technology for $D$. melanogaster embryos suggests the investigation of effects of specific disruptions to the expression of JHE by mutagenesis of the regulatory region of the JHE gene.

\subsection{Conclusions}

This study has identified two specific JH hydrolysing enzymes in D. melanogaster, JHE and JHEH. One of the enzymes, JHE, is highly specific for JHIII and $\mathrm{JHB}_{3}$, the two isoforms of $\mathrm{JH}$ produced by the higher Diptera. The specificity of these enzymes and their temporal and spatial regulation indicate that dipteran $\mathrm{JH}$ hydrolysis, possibly modulated by interaction with the $\mathrm{JH}$ carrier, acts in a coordinated fashion with $\mathrm{JH}$ synthesis to regulate $\mathrm{JH}$ titre. Furthermore, the pattern of $\mathrm{JH}$ hydrolysis during metamorphosis closely matches the lepidopteran model (Roe and Venkatesh, 1990), except for the lack of prewandering, haemolymph JHE. These results suggest that the role of $\mathrm{JH}$ hydrolysing enzymes in Diptera is essentially similar to their role in other insects. 


\section{References}

Abdel-Aal, Y. A. I. and Hammock, B. D. (1985) Apparent multiple catalytic sites involved in the ester hydrolysis of juvenile hormones by the hemolymph and by an affinitypurified esterase from Manduca sexta Johannson (Lepidoptera: Sphingidae). Arch. Biochem. Biophys. 243, 206-219.

Abdel-Aal, Y. A. I. and Hammock, B. D. (1986) Transition state analogs as ligands for affinity purification of juvenile hormone esterase. Science 233, 1073-1076.

Abdel-Aal, Y. A. I. and Hammock, B. D. (1988) Kinetics of binding and hydrolysis of juvenile hormone II in the hemolymph of Trichoplusia ni (Hübner). Insect Biochem. 18, 743-750.

Abdel-Aal, Y. A. I., Hanzlik, T. N., Hammock, B. D., Harshman, L. G. and Prestwich, G. (1988) Juvenile hormone esterases in two Heliothines: kinetic, biochemical and immunogenic characterisation. Comp. Biochem. Physiol. 90B, 117-124.

Adams, T. S. and Gerst, J. W. (1992) Interaction between diet and hormones on vitellogenin levels in the housefly, Musca domestica. Invert. Reprod. Develop. 21, 91-98.

Ajami, A. M. and Riddiford, L. M. (1973) Comparitive metabolism of the cecropia juvenile hormone. J. Insect Physiol. 19, 635-645.

Altaratz, M., Applebaum, S. W., Richard, D. S., Gilbert, L. I. and Segal, D. (1991) Regulation of juvenile hormone synthesis in wild-type and apterous mutant Drosophila. Mol. Cell. Endocrinol. 81, 205-216.

Anderson, D. T. (1964) The larval development of Dacus tryoni (Frogg.) (Diptera: Trypetidae) II. Development of imaginal rudiments other than the principal discs. Aust. J. Zool. 12, 1-8.

Andres, A. J. and Thummel, C. S. (1992) Hormones, puffs and flies - the molecular control of metamorphosis by ecdysone. Trends Genet. 8, 132-138.

Bainbridge, S. P. and Bownes, M. (1981) Staging the metamorphosis of Drosophila melanogaster. J. Embryol. Exp. Morphol. 66, 57-80.

Baker, F. C., Tsai, L. W., Reuter, C. C. and Schooley, D. A. (1987) In vivo fluctuations of $\mathrm{JH}, \mathrm{JH}$ acid, and ecdysteroid titer, and JH esterase activity during development of fifth stadium Manduca sexta. Insect Biochem. 17, 989-996.

Bakker, K. (1959) Feeding period, growth, and pupation in larvae of Drosophila melanogaster. Ent. Exp. Appl. 2, 171-186.

Bennett, J. P. and Yamamura, H. I. (1985) Neurotransmitter, hormone, or drug receptor binding methods. In Neurotransmitter Receptor Binding (Edited by Yamamura, H. I., Enna, S. J. and Kuher, M. J.), pp. 61-89. Raven Press, New York.

Berger, E. and Canter, R. (1973) The esterases of Drosophila 1. The anodal esterases and their possible role in eclosion. Develop. Biol. 33, 48-55. 
Berger, E. M. and Canter, R. (1972) "Moulting" esterases of Drosophila. Dros. Inf. Serv. 49, 90.

Besson, M. T., Delbecque, J. P., Mathelin, J., Boisson, A. M. and Delachambre, J. (1986) Epidermal polyamine levels related to cell cycle events during metamorphosis of Tenebrio molitor. (Insecta, Coleoptera): effect of juvenoid application. Comp. Biochem. Physiol. 83B, 589-593.

Bhaskaran, G. (1972) Inhibition of imaginal differentiation in Sarcophaga bullata by juvenile hormone. J. Exp. Zool. 182, 127-142.

Birnbaum, M. J. and Gilbert, L. I. (1990) Juvenile hormone stimulation of ornithine decarboxylase activity during vitellogenesis in Drosophila melanogaster. J. Comp. Physiol. 160B, 145-151.

Birnbaum, M. J., Whelan, T. M. and Gilbert, L. I. (1988) Temporal alterations in polyamine content and ornitine decarboxylase activity during the larval-pupal development of Manduca sexta. Insect Biochem. 18, 853-859.

Bisser, A. and Emmerich, H. (1981) Hydrolysis of juvenile hormone and juvenile hormone analogues in the larval integument of Drosophila hydei. In Juvenile Hormone Biochemistry (Edited by Pratt, G. E. and Brooks, G. T.), pp. 199-212. Elsevier/North-Holland Biomedical Press, Amsterdam.

Bollenbacher, W. E. (1988) The interendocrine regulation of larval-pupal development in the tobacco hornworm, Manduca sexta: a model. J. Insect Physiol. 34, 941-947.

Bonaric, J. C. and Juberthie, C. (1990) Juvenile hormone-like compounds in terrestrial Chelicerata (Arachnida). In Morphogenic Hormones of Arthropods (Edited by Gupta, A. P.), Vol. 1, pp. 61-72. Rutgers University Press, New Brunswick.

Booth, T. F., Bonning, B. C. and Hammock, B. D. (1992) Localisation of juvenile hormone esterase during development in normal and recombinant baculavirusinfected larvae of the moth Trichoplusia ni. Tissue and Cell 24, 267-282.

Borovsky, D., Carlson, D. A. and Ujváry, I. (1992) In vivo and in vitro biosynthesis and metabolism of methyl farnesoate, juvenile hormone-III, and juvenile hormone-III acid in the mosquito Aedes aegypti.J. Med. Entomol. 29, 619-629.

Borst, D. W. and Tsukimra, B. (1992) Methyl farnesoate levels in crustaceans. In Insect Juvenile Hormone Research (Edited by Mauchamp, B., Couillaud, F. and Baehr, J. C.), pp. 27-35. Institut National de la Recherche Agronomique, Paris.

Bowers, W. S. (1982) Endocrine strategies for insect control. Ent. exp. \& appl. 31, 3-14.

Bownes, M. (1989) The role of juvenile hormone, ecdysone and the ovary in the control of Drosophila vitellogenesis. J. Insect Physiol. 35, 409-413.

Bownes, M. and Rembold, H. (1987) The titre of juvenile hormone during the pupal and adult stages of the life cycle of Drosophila melanogaster. Eur. J. Biochem. 164, 709-712.

Bradford, M. M. (1976) A rapid and sensitive method for the quantitation of microgram quantities of protein using the principle of dye binding. Anal. Biochem. 72, 248254. 
Bührlen, U., Emmerich, H. and Rembold, H. (1984) Titre of juvenile hormone III in Drosophila hydei during metamorphosis determined by GC-MS-MIS. $Z$. Naturforsch. 39c, 1150-1154.

Casas, J., Harshman, L. G., Messeguer, A., Kuwano, E. and Hammock, B. D. (1991) In vitro metabolism of juvenile hormone III and juvenile hormone III bisepoxide by Drosophila melanogaster and mammalian cytosolic epoxide hydrolase. Arch Biochem. Biophys. 286, 153-158.

Cherbas, P. (1993) The IVth Karlson lecture: ecdysone-responsive genes. J. Insect Biochem. Molec. Biol. 23, 2-11.

Chihara, C. J., Petri, W. H., Fristrom, J. W. and King, D. S. (1972) The assay of ecdysones and juvenile hormones on Drosophila imaginal discs in vitro. J. Insect Physiol. 18, 1115-1123.

Cleland, W. W. (1979) Statistical analysis of enzyme kinetic data. Methods Enzymol. 63, 103-138.

Connat, J. L. (1983) Juvenile hormone esterase activity during the last larval and pupal stages of Tenebrio molitor. J. Insect Physiol. 29, 515-521.

Cooke, P. H., Richmond, R. C. and Oakeshott, J. G. (1987) High resolution electrophoretic variation at the esterse- 6 locus in a natural population of Drosophila melanogaster. Heredity 59, 259-264.

Couldron, T. A., Dunn, P. E., Seballos, H. L., Wharen, R. E., Sanberg, L. L. and Law, J. H. (1981) Preparation of homogeneous juvenile hormone specific esterase from the haemolymph of the tobacco hornworm, Manduca sexta. Insect Biochem.11, 453461.

Croston, G., Abdel-Aal, Y. A. I., Gee, S. J. and Hammock, B. D. (1987) Activation of crude and homogeneous juvenile hormone esterases by organic solvents. Insect Biochem. 17, 1017-1021.

Cusson, M., Yagi, K. J., Ding, Q., Duve, H., Thorpe, A., McNeil, J. N. and Tobe, S. S. (1991) Biosynthesis and release of juvenile hormone and its precursors in insects and crustaceans: the search for a unifying arthropod endocrinology. Insect Biochem. 21, 1-6.

Cygler, M., Schrag, J. D., Sussman, J. L., Harel, M., Silman, I., Gentry, M. K. and Doctor, B. P. (1993) Relationship between sequence conservation and three-dimensional structure in a large family of esterases, lipases, and related proteins. Prot. Sci. 2, 366-382.

Cymborowski, B., Bogus, M., Beckage, N. E., Williams, C. M. and Riddiford, L. M. (1982) Juvenile hormone titres and metabolism during starvation-induced supernumary larval moulting of the tobacco hornworm, Manduca sexta L. J. Insect Physiol. 28, 129-135.

Dai, J. and Gilbert, L. I. (1991) Metamorphosis of the corpus allatum and degeneration of the prothoracic glands during the larval-pupal-adult transformation of Drosophila melanogaster: a cytophysiological analysis of the ring gland. Develop. Biol. 144, 309-326. 
de Bianchi, A. G., de L. Capurro, M. and Marinotti, O. (1987) Lipophorin in the larval and adult stages of Musca domestica. Arch. Insect Biochem. Physiol. 6, 39-48.

de Kort, C. A. D. (1990) Thirty-five years of diapause research with the Colorado potato beetle. Entomol. exp. appl.56, 1-13.

de Kort, C. A. D. and Granger, N. A. (1981) Regulation of the juvenile hormone titre. Ann. Rev. Entomol. 26, 1-28.

de Kort, C. A. D., Peter, M. G. and Koopmanschap, A. B. (1983) Binding and degradation of juvenile hormone III by haemolymph of the Colorado potato beetle: a re-examination. Insect Biochem. 13, 481-487.

de Kort, C. A. D., Wieten, M. and Kramer, S. J. (1979) The occurence of juvenile hormone specific esterases in insects. A comparative study. 82, 325-331.

Denlinger, D. A. and Tanaka, S. (1989) Cycles of juvenile hormone esterase activity during the juvenile hormone-driven cycles of oxygen consumption in diapause of flesh flies. Experientia 45, 474-476.

Devereux, J., Haeberli, P. and Smithies, O. (1984) A comprehensive set of sequence analysis programs for the VAX. Nucleic Acids Res. 12, 387-395.

Dominick, O. S. and Truman, J. W. (1985) The physiology of wandering behaviour in Manduca sexta II. the endocrine control of wandering behaviour. J. Exp. Biol. 117, 45-68.

Duve, H., Thorpe, A., Yagi, K. J., Yu, C. G. and Tobe, S. S. (1992) Factors affecting the biosynthesis and release of juvenile hormone bisepoxide in the adult blowfly Calliphora vomitoria. J. Insect Physiol. 38, 575-585.

East, P. D. (1982) Non-specific esterases of Drosophila buzzatii. In Ecological Genetics and Evolution (Edited by Barker, J. S. F. and Starmer, W. T.), pp. 323-338. Academic Press Australia, North Ryde.

Engelmann, F. (1984) Juvenile hormone binding compounds in hemolymph and tissues of an insect: the functional significance. In Advances in Invertebrate Reproduction (Edited by Engels, W., Clark, W. H., Fischer, A., Olive, P. J. W. and Went, D. F.), Vol. 3, pp. 177-187. Elsevier, Amsterdam.

Engelmann, F., Mala, J. and Borst, D. (1988) Juvenile hormone-binding protein and juvenile hormone esterase activity in hemolymph from last-instar nymphs of Leucophaea maderae. Arch. Insect Biochem. Physiol. 8, 11-23.

Fescemyer, H. W., Rose, R. L., Sparks, T. C. and Hammond, A. M. (1986) Juvenile hormone esterase activity in developmentally synchronous ultimate stadium larvae of the migrant insect Anticarsia gemmatalis. J. Insect Physiol. 32, 1055-1063.

Fournier, D., Bride, J., Karch, F. and Bergé, J. (1988) Acetylcholinesterase from Drosophila melanogster. Febs Lett. 238, 333-337.

Fraenkel, G. and Bhaskaran, G. (1973) Pupariation and pupation in cyclorraphous flies (Diptera): terminology and interpretation. Ann. Entomol. Soc. Am. 66, 418-422. 
Giese, C., Spindler, K. D. and Emmerich, H. (1976) The solubility of insect juvenile hormone in aqueous solutions and its adsorption by glassware and plastics. $Z$. Naturforsch. 32c, 158-160.

Goodman, W. G. (1990) Biosynthesis, titre reglation, and transport of juvenile hormones. In Morphogenic Hormones of Arthropods (Edited by Gupta, A. P.), Vol. 1, pp. 83124. Rutgers University Press, New Brunswick.

Gu, S. H., Tsai, R. S., Chow, Y. S. and Lin, F. J. (1992) Sexual dimorphism in developmental rate and ecdysteroid titre in Orgyia postica. J. Insect Physiol. 38, 1043-1049.

Gunawan, S. and Engelmann, F. (1984) Esterolytic degradation of juvenile hormone in the haemolymph of the adult female of Leucophaea maderae. Insect Biochem. 14, 610-607.

Gupta, A. P. (Ed.)(1990a) Morphogenic Hormones of Arthropods Rutgers University Press, New Brunswick.

Gupta, A. P. (1990b) Morphogenic hormones and their glands in arthropods: evolutionary aspects. In Morphogenic Hormones of Arthropods (Edited by Gupta, A. P.), Vol. 1, pp. 1-34. Rutgers University Press, New Brunswick.

Hales, D. F. and Mittler, T. E. (1988) Male production by aphids prenatally treated with precocene: prevention by short-term kinoprene treatment. Arch. Insect Biochem. Physiol. 7, 29-36.

Hammock, B. D. (1985) Regulation of juvenile hormone titre: Degradation. In Comprehensive Insect Physiology, Biochemistry and Pharmacology (Edited by Kerkut, G. A. and Gilbert, L. I.), Vol. 2, pp. 431-472. Pergamon Press, New York.

Hammock, B. D., Abdel-Aal, Y. A. I., Hanzlik, T. N., Croston, G. E. and Roe, R. M. (1987) Affinity purification and characteristics of juvenile hormone esterase from Lepidoptera. In Molecular Entomology (Edited by Law, J. H.), pp. 315-328. Liss, New York.

Hammock, B. D., Abdel-Aal, Y. A. I., Mullin, C. A., Hanzlik, T. N. and Roe, R. M. (1984) Substituted thiotrifluoropropanones as potent selective inhibitors of juvenile hormone esterase. Pestic. Biochem. Physiol. 22, 209-223.

Hammock, B. D. and Roe, R. M. (1985) Analysis of juvenile hormone esterase activity. Methods Enzymol. 111, 487-494.

Hanzlik, T. N. (1988) The physiology, biochemistry and molecular biology of juvenile hormone esterase: development of its potential as an insecticidal agent, Ph.D. Dissertation, University of California, Davis, U. S. A.

Hanzlik, T. N., Abdel-Aal, Y. A. I., Harshman, L. G. and Hammock, B. D. (1989) Isolation and sequencing of cDNA clones coding for juvenile hormone esterase from Heliothis virescens. Evidence for a catalytic mechanism for the serine carboxylesterases different from that of the serine proteases. J. Biol. Chem. 264, 12419-12425.

Hanzlik, T. N. and Hammock, B. D. (1987) Characterisation of affinity-purified juvenile hormone esterase from Trichoplusia ni.J. Biol. Chem. 262, 13584-13591. 
Hanzlik, T. N. and Hammock, B. D. (1988) Characterisation of juvenile hormone hydrolysis in early larval development of Trichoplusia ni. Arch. Insect Biochem. Physiol. 9, 135-156.

Harshman, L. G., Casas, J., Dietze, E. C. and Hammock, B. D. (1991) Epoxide hydrolase activities in Drosophila melanogaster. Insect Biochem. 21, 887-894.

Hartfelder, K., de Oliveira Tozetto, S. and Rachinsky, A. (1993) Sex-specific developmental profiles of juvenile hormone synthesis in honey bee larvae. Roux's Arch. Dev. Biol. 202, 176-180.

Hayakawa, Y. (1990) Juvenile hormone esterase activity repressive factor in the plasma of parasitized insect larvae. J. Biol. Chem. 265, 10813-10816.

Hayakawa, Y. (1991) Structure of a growth-blocking peptide present in parasitized insect hemolymph. J. Biol. Chem. 266, 7982-7984.

Hayakawa, Y. (1992) A putitive new juvenile peptide hormone in lepidopterous insects. Biochem. Biophys. Res. Comm. 185, 1141-1147.

Hayakawa, Y. and Yasuhara, Y. (1993) Growth-blocking peptide or polydnavirus effects on the last larval instar larvae of some insect species. Insect Biochem. Molec. Biol. 23, 225-231.

Healy, M. J., Dumancic, M. M. and Oakeshott, J. G. (1991) Biochemical and physiological studies of soluble esterases from Drosophila melanogaster. Biochem. Genet. 29, 365-388.

Healy, M. J., Dumancic, M. M., Cao, A. and Oakeshott, J. G. (in preparation) Evolution of sequences conferring sex-specific expression on Drosophila melanogaster esterase 6.

Hearlt, A. J., Rickards, R. W., Thomas, R. D. and East, P. D. (1993) The absolute configuration of juvenile hormone III bisepoxide. J. Chem. Soc. Chem. Comm. in press.

Hooper, G. H. S. (1976) Esterase mediated hydrolysis of naphthyl esters, malathion, methoprene, and cecropia juvenile hormone in Culex pipiens pipiens. Insect Biochem. 6, 255-266.

Ichinose, R., Kamita, S. G., Maeda, S. and Hammock, B. D. (1992a) Pharmacokinetic studies of the recombinant juvenile hormone esterase in Manduca sexta. Pestic. Biochem. Physiol. 42, 13-23.

Ichinose, R., Nakamura, A., Yamoto, T., Booth, T. F., Maeda, S. and Hammock, B. D. (1992b) Uptake of juvenile hormone esterase by pericardial cells of Manduca sexta. Insect Biochem. Molec. Biol. 22, 893-904.

Jackson, J. B., Thompson, H. M. and Walker, C. H. (1992) The affinity purification of serum carboxylesterase from pidgeons Columba livia. Pestic. Sci. 36, 75-78.

Jesudason, P., Anspaugh, D. D. and Roe, R. M. (1992) Juvenile hormone metabolism in the plasma, integument, midgut, fat body, and brain, during the last instar of the tobacco hornworm, Manduca sexta (L). Arch. Insect Biochem. Physiol. 20, 87-105. 
Jesudason, P., Venkatesh, K. and Roe, R. M. (1990) Haemolymph juvenile hormone esterase during the life cycle of the tobacco hornworm, Manduca sexta (L.). Insect Biochem. 20, 593-604.

Jones, D., Jones, G., Click, A., Rudnicka, M. and Sreekrishna, S. (1986) Multiple forms of juvenile hormone esterase active sites in the hemolymph of larvae of Trichoplusia ni. Comp. Biochem. Physiol. 85B, 773-781.

Jones, D., Jones, G. and Hammock, B. D. (1981) Growth parameters associated with endocrine events in larval Trichoplusia ni (Hübner) and timing of these events with developmental markers. J. Insect Physiol. 27, 779-788.

Jones, D., Jones, G., Wing, K. D., Rudnicka, M. and Hammock, B. D. (1982) Juvenile hormone esterases of Lepidoptera I. Activity in the hemolymph during the last larval instar of 11 species. J. Comp. Physiol. 148, 1-10.

Jones, G. (1985) The role of juvenile hormone esterase in terminating larval feeding and initiating metamorphic development in Trichoplusia ni. Entomol. exp. appl.39, 171-176.

Jones, G. (1986) Studies on the regulation, function and properties of juvenile hormone esterases of Trichoplusia ni. In Insect Neurochemistry and Neurophysiology (Edited by Borkovec, A. B. and Gelman, D. B.), pp. 315-318. Humana Press, Clifton.

Jones, G. and Click, A. (1987) Developmental regulation of juvenile hormone esterase in Trichoplusia ni: its multiple electrophoretic forms occur during each larval ecdysis. J. Insect Physiol. 33, 207-213.

Jones, G. and Hammock, B. D. (1985) Critical roles for juvenile hormone and its esterase in the prepupa of Trichoplusia ni. Arch. Insect Biochem. Physiol. 2, 397-404.

Jones, G., Hanzlik, T., Hammock, B. D., Schooley, D. A., Miller, C. A., Tsai, L. W. and Baker, F. C. (1990) The juvenile hormone titre during the penultimate and ultimate larval stadia of Trichoplusia ni. J. Insect Physiol. 36, 77-83.

Kelly, T. J., Adams, T. S., Schwartz, M. B., Birnbaum, M. J., Rubenstein, E. C. and Imberski, R. B. (1987) Juvenile hormone and ovarian maturation in Diptera: a review of recent results. Insect Biochem. 17, 1089-1093.

Kiguchi, K. and Riddiford, L. M. (1978) A role of juvenile hormone in pupal development of the tobacco hornworm, Manduca sexta. J. Insect Physiol. 24, 673680.

Kindle, H., Winistorfer, M., Lanzrein, B. and Mori, K. (1989) Relationship between the absolute configuration and the biological activity of juvenile hormone III. 45, 356360.

King, L. E. and Tobe, S. S. (1993) Changes in the titre of a juvenile hormone III binding lipophorin in the haemolymph of Diploptera punctata during development and reproduction: functional significance. J..Insect Physiol. 39, 241-251.

Klages, G. and Emmerich, H. (1979) Juvenile hormone metabolism and juvenile hormone esterase titre in hemolymph and peripheral tissues of Drosophila hydei.J. Comp. Physiol. 132, 319-325. 
Kochman, M. and Wieczorek, E. (1991) Molecular mechanism of the juvenile hormone action. Acta Biochemica Polonica 38, 393-405.

Koeppe, J. K., Fuchs, M., Chen, T. T., Hunt, L. M., Kovalick, G. E. and Briers, T. (1985) The role of juvenile hormone in reproduction. In Comprehensive Insect Physiology, Biochemistry, and Pharmacology (Edited by Kerkut, G. A. and Gilbert, L. I.), Vol. 8, pp. 165-203. Pergamon Press, New York.

Kramer, S. J. and de Kort, C. A. D. (1976) Some properties of hemolymph esterases from Leptinotarsa decemlineata say. Life Sci. 19, 211-218.

Kumaran, A. K. (1990) Modes of action of juvenile hormones at cellular and molecular levels. In Morphogenic Hormones of Arthropods (Edited by Gupta, A. P.), Vol. 1, pp. 181-227. Rutgers University Press, New Brunswick.

Laemmli, U. K. (1970) Cleavage of structural proteins during the assembly of the head of bacteriophage T4. Nature 227, 680-695.

Lanzrein, B., Wilhelm, R. and Riechsteiner, R. (1993) Differential degradation of racemic and 10R-juvenile hormone III by cockroach (Nauphoeta cinerea) haemolymph and the use of lipophorin for long-term culturing of corpora allata. J. Insect Physiol. 39, 53-63.

Laufer, H., Wainwright, G., Young, N. J., Sagi, A., Ahl, J. S. B. and Rees, H. H. (1993) Ecdysteroids and juvenoids in two male morphotypes of Libinia emarginata. $J$. Insect Biochem. Molec. Biol. 23, 171-174.

LeFevere, K. S., Lacey, M. J., Smith, P. H. and Roberts, B. (1993) Identification and quantification of juvenile homone biosynthesised by larval and adult Australian sheep blowfly Lucilia cuprina (Diptera: Calliphoridae). Insect Biochem. Mol. Biol. in press.

Linderman, R. J., Leazer, J., Venkatesh, K. and Roe, R. M. (1987) The inhibition of juvenile hormone esterase by trifluoromethylketones: steric parameters at the active site. Pestic. Biochem. Physiol. 29, 266-277.

Linderman, R. J., Upchurch, L., Lonicar, M., Venkatesh, K. and Roe, R. M. (1989) Inhibition of insect juvenile hormone esterase by $\alpha, \beta$-unsaturated and $\alpha$-acetylenic trifluoroketones. Pestic. Biochem. Physiol. 35, 291-299.

Linderman, R. J., Graves, D. M., Garg, S., Venkatesh, K., Anspaugh, D. D. and Roe, R. M. (1993) Unique inhibition of a serine esterase. Tetrahedron Lett. 34, 3227-3230.

Ludwig, M. Z., Tamarina, N. A. and Richmond, R. C. (1993) Localization of sequences controlling the spatial, temporal, and sex-specific expression of the esterase 6 locus in Drosophila melanogaster adults. Proc. Natl. Acad. Sci. USA. in press.

Madhavan, M. M. and Schneiderman, H. A. (1977) Histological analysis of the dynamics of growth of imaginal discs and histoblast nests during the larval development of Drosophila melanogaster. Roux's Arch. Dev. Biol. 183, 269-305.

Mane, S. D., Tepper, C. S. and Richmond, R. C. (1983) Studies of esterase-6 in Drosophila melanogaster XIII: purification and characterisation of the two major isozymes. Biochem. Genet. 21, 1019-1040. 
Markwell, M. A., Haas, S. M., Beiber, L. L. and Tolbert, N. E. (1978) A modification of the Lowry procedure to simplify protein determinations in membrane and lipoprotein samples. Anal. Biochem. 87, 206.

Mastrapaolo, W. and Yourno, J. (1981) An ultraviolet spectrophotometric assay for $\alpha$ naphthylacetate and $\alpha$-naphthylbutyrate esterases. Analyt. Biochem. 115, 188-193.

Merril, C. R., Goldman, D., Sedman, S. A. and Ebert, M. H. (1980) Ultrasensitive stain for proteins in polyacrylamide gels shows regional variation in cerebrospinal fluid proteins. Science 211, 1437-1438.

Meyer, W. R. and Lanzrein, B. (1989) Degradation of juvenile hormone and methylation of juvenile hormone acid by corpora cardiaca-corpora allata of the cockroach, Nauphoeta cinerea: II. Physiological aspects. Arch. Insect Biochem. Physiol. 10, 317-331.

Mitchell, H. K. and Mitchell, A. (1964) Mass culture and age selection in Drosophila. Dros. Inf. Serv. 39, 135.

Mumby, S. M., Hammock, B. D., Sparks, T. C. and Ota, K. (1979) Synthesis and bioassay of carbamate inhibitors of the juvenile hormone hydrolysing esterases from the house fly, Musca domestica. J. Agric. Food Chem. 27, 763-765.

Myers, M., Richmond, R. C. and Oakeshott, J. G. (1988) On the origins of esterases. Mol. Biol. Evol. 5, 113-119.

Myers, M. A. (1990) Structure/function relationships of the esterase 6 protein of Drosophila mealnogaster, Ph. D. thesis, Australian National University.

Newitt, R. A. and Hammock, B. D. (1986) Relationship between juvenile hormone and ecdysteroids in larval-pupal development of Trichoplusia $n i$ (Lepidoptera: Noctuiidae). J. Insect Physiol. 32, 835-844.

Nijhout, H. F. (1975) Dynamics of juvenile hormone action in larvae of the tobacco hornworm, Manduca sexta (L.). Biol. Bull. 149, 568-579.

Numata, H., Numata, A., Takahashi, C., Nakagawa, Y., Iwatani, K., Takahashi, S., Miura, K. and Chinzei, Y. (1992) Juvenile hormone-I is the principal juvenile-hormone in a hemipteran insect, Riptortus clavatus. Experientia 48, 606-610.

Oakeshott, J. G., Collet, C., Phillis, R. W., Neilsen, K. M., Russell, K. J., Chambers, G. K., Ross, V. and Richmond, R. C. (1987) Molecular cloning and characterisation of esterase-6, a serine hydrolase of Drosophila. Proc. Natl. Acad. Sci. USA 84, 33593363.

Oakeshott, J. G., Healy, M. J. and Game, A. Y. (1990) Regulatory evolution of B-carboxyl esterases in Drosophila. In Ecological and Evolutionary Genetics of Drosophila (Edited by Barker, J. S. F., Starmer, W. T. and MacIntyre), pp. 359-387. Plenum Press, New York.

Ollis, D. L., Cheah, E., Cygler, M., Dijkstra, B., Frolow, F., Franken, S. M., Harel, M., Remington, S. J., Silman, I., Schrag, J., Sussman, J. L., Verschueren, K. H. G. and Goldman, A. (1992) The $\alpha / \beta$ hydrolase fold. Prot. Eng. 5, 197-211. 
Osterbur, D. L. and Steiner, W. W. M. (1979) Pupal specific esterase of Drosophila psuedoobscura. Genetics 91, s92.

Ottea, J. A., Harshman, L. G. and Hammock, B. D. (1987) Patterns of epoxide metabolism by epoxide hydrolase and glutathione S-transferase associated with age and genotype in Drosophila melanogaster. Mutation Res. 177, 247-254.

Ottea, J. A., Harshman, L. G. and Hammock, B. D. (1988) Novel assay for determining the metabolic fate of juvenile homone III: A study with Drosophila melanogaster. Arch. Insect Biochem. Physiol. 8, 25-37.

Ozols, J. (1987) Isolation and characterisation of a 60-kilodalton glycoprotein esterase from liver microsomal membranes. J. Biol. Chem. 262, 15316-15321.

Ozyhar, A., Wisniewski, J. R., Sehnal, F. and Kochman, M. (1983) Age dependent changes in the binding and hydrolysis of juvenile hormone in the haemolymph of last larval instar of Galleria mellonella. Insect Biochem. 13, 435-441.

Palli, S. R., McClelland, S., Hiruma, K., Latli, B. and Riddiford, L. M. (1991a) Developmental expression and hormonal regulation of the nuclear $29 \mathrm{kDa}$ juvenile hormone-binding protein in Manduca sexta larval epidermis. J. Exp. Zool. 260, 337-344.

Palli, S. R., Riddiford, L. M. and Hiruma, K. (1991b) Juvenile hormone and "retinoic acid" receptors in Manduca epidermis. Insect Biochem. 21, 7-15.

Pearson, M. J. (1972) Imaginal discs and the abdominal histoblasts of Calliphora erythrocephala (Diptera). Nature 238, 349-351.

Peter, M. G., Gunawan, S., Gellissen, G. and Emmerich, H. (1979) Differences in hydrolysis and binding of homologous juvenile hormones in Locusta migratoria hemolymph. Z. Naturforsch. 34c, 588-598.

Philpott, M. L. and Hammock, B. D. (1990) Juvenile hormone esterase is a biochemical anti-juvenile hormone agent. Insect Biochem. 20, 451-459.

Pongs, O. (1988) Ecdysteroid regulated gene expression in Drosophila melanogaster. Eur. J. Biochem. 175, 199-204.

Poodry, C. A. and Woods, D. F. (1990) Control of the developmental timer for Drosophila pupariation. Roux's Arch. Dev. Biol. 199, 219-227.

Postlethwait, J. H. (1974) Juvenile hormone and the adult development of Drosophila. Biol. Bull. 147, 119-135.

Pratt, G. E. (1975) Inhibition of juvenile hormone carboxyesterase of locust haemolymph by organophosphates in vitro. Insect Biochem. 5, 595-607.

Rauschenbach, I. Y., Golosheikina, L. B., Korochkina, L. S. and Korochkin, L. I. (1977) Genetics of esterases in Drosophila. V. Characteristics of the "pupal" esterase in D. virilis. Biochem. Genet. 15, 531-548.

Rauschenbach, I. Y., Lukashina, N. S., Budker, V. G. and Korochkin, L. I. (1987) Genetics of esterases in Drosophila. IX. Characterization of the JH-esterase in D. virilis. Biochem. Genet. 25, 687-704. 
Rauschenbach, I. Y., Lukashina, N. S., Khlebodarova, T. M. and Korochkin, L. I. (1991) Role of juvenile hormone esterase in diptera (Drosophila virilis) metamorphosis. $J$. Insect Physiol. 37, 541-548.

Renucci, M., Martin, N. and Strambi, C. (1984) Temporal variations of hemolymph esterase activity and juvenile hormone titres during ovocyte maturation in Acheta domesticus (Orthoptera). Gen. Comp. Endocrinol. 55, 480-487.

Richard, D. S., Applebaum, S. W. and Gilbert, L. I. (1989a) Developmental regulation of juvenile hormone biosynthesis by the ring gland of Drosophila melanogaster. $J$. Comp. Physiol. 159, 383-387.

Richard, D. S., Applebaum, S. W. and Gilbert, L. I. (1990) Allostatic regulation of juvenile hormone production in vitro by the ring gland of Drosophila melanogaster. Mol. Cell. Endocrinol. 68, 153-161.

Richard, D. S., Applebaum, S. W., Sliter, T. J., Baker, F. C., Schooley, D. A., Reuter, C. C., Henrich, V. C. and Gilbert, L. I. (1989b) Juvenile hormone bisepoxide biosynthesis in vitro by the ring gland of Drosophila melanogaster: A putative juvenile hormone in the higher Diptera. Proc. Natl. Acad. Sci. 86, 1421-1425.

Richard, D. S. and Gilbert, L. I. (1991) Reversible juvenile hormone inhibition of ecdysteroid and juvenile hormone synthesis by the ring gland of Drosophila melanogaster. Experientia 47, 1063-1066.

Richards, G. (1978) Sequential gene activation by ecdysone in polytene chromosomes of Drosophila melanogaster. VI. Inhibition by juvenile hormones. Dev. Biol. 66, 3242.

Rickards, R. W. and Thomas, R. D. (1992) Synthesis of four stereoisomers of the higher dipteran juvenile hormone III bisepoxide. Tetrahedron Lett. 33, 8137-8140.

Riddiford, L. M. (in press) Hormones and Drosophila development. In The Development of Drosophila (Edited by Bate, M. and Martinez-Arias, A.), Cold Spring Harbor Laboratory Press, New York.

Riddiford, L. M. and Ashburner, M. (1991) Effects of juvenile hormone mimics on larval development and metamorphosis of Drosophila melanogaster. Gen. Comp. Endocrinology 82, 172-183.

Roberts, B. and Gilbert, L. I. (1986) Ring gland and prothoracic gland sensitivity to interspecific prothoracicotropic hormone extracts. J. Comp. Physiol. B 156, 767771.

Roe, R. M., Linderman, R. J., Lonikar, M., Venkatesh, K., Abdel-Aal, Y. A. I., Leazer, J. and Upchurch, L. (1990) Rational design and synthesis of polarized ketones as inhibitors of juvenile hormone esterase: importance of juvenile hormone mimicry. J. Agric. Food Chem. 38, 1274-1278.

Roe, R. M. and Venkatesh, K. (1990) Metabolism of juvenile hormones: degradation and titre regulation. In Morphogenic Hormones of Arthropods (Edited by Gupta, A. P.), Vol. 1, pp. 125-180. Rutgers University Press, New Brunswick. 
Roseland, C. R. and Schneiderman, H. A. (1979) Regulation and metamorphosis of the abdominal histoblasts of Drosophila melanogaster. Wilh. Roux's Arch. Dev. Biol. 186, 235-265.

Rudnicka, M. and Jones, D. (1987) Characterisation of homogeneous juvenile hormone esterase from larvae of Trichoplusia ni. Insect Biochem. 17, 373-382.

Rudnicka, M. and Kochman, M. (1984) Purification of the juvenile hormone esterase from the haemolymph of the wax moth Galleria mellonella (Lepidoptera). Insect Biochem. 14, 189-198.

Sanburg, L. L., Kramer, K. J., Kezdy, F. J. and Law, J. H. (1975) Role of juvenile hormone esterases and carrier proteins in insect development. Nature 253, 266-267.

Satyanarayana, K., Bhaskaran, G., Dahm, K. H. and Meola, R. (1992) Regulation of vitellogenin synthesis by juvenile hormone in the corn earworm, Helicoverpa zea. Invert. Reprod. Develop. 21, 169-178.

Saunders, D. S., Richard, D. S., Applebaum, S. W., Ma, M. and Gilbert, L. I. (1990) Photoperiodic diapause in Drosophila melanogaster involves a block to the juvenile hormone regulation of ovarian maturation. Gen. Comp. Endrocrinol. 79, 174-184.

Schooley, D. A., Baker, F. C., Tsai, L. W., Miller, C. A. and Jamieson, G. C. (1984) Juvenile hormones 0, I, and II exist only in Lepidoptera. In Biosynthesis, Metabolism and Mode of Action of Invertebrate Hormones (Edited by Hoffman, J. and Porchet, M.), pp. 373-383. Springer-Verlag, Berlin Heidelberg.

Schooley, D. A. and Baker, F. C. (1985) Juvenile hormone biosynthesis. In Comprehensive Insect Physiology, Biochemistry and Pharmacology (Edited by Kerkut, G. A. and Gilbert, L. I.), Vol. 2, pp. 363-389. Pergamon Press, New York.

Schwartz, M. B., Imberski, R. B. and Kelly, T. J. (1984) Analysis of metamorphosis in Drosophila melanogaster: characterization of giant, an ecdysteroid-deficient mutant. Develop. Biol. 103, 85-95.

Schwieter-Peyer, B. (1973) Comparison of the effect of the geometrical isomers of juvenile hormone on Blattella germanica, Dysdercus cingulatus, and Sitophilus granarius. Insect Biochem. 3, 275-282.

Segraves, W. A. (1991) Something old, something new: the steroid receptor superfamily in Drosophila. Cell 67, 225-228.

Sehnal, F. and Zdarek, J. (1976) Action of juvenoids on the metamorphosis of cyclorrhaphous Diptera. J. Insect Physiol. 22, 673-682.

Sevala, V. L. and Davey, K. G. (1989) Action of juvenile hormone on the follicle cells of Rhodnius prolixus: Evidence for a novel regulatory mechanism involving protein kinase C. Experientia 45, 355-356.

Shaaya, E. (1993) Interference of the insect growth regulator methoprene in the process of larval-pupal differentiation. Arch. Insect Biochem. Physiol. 22, 233-243.

Shapiro, A. B., Wheelock, G. D., Hagedorn, H. H., Baker, F. C., Tsai, L. W. and Schooley, D. A. (1986) Juvenile hormone and juvenile hormone esterase in adult females of the mosquito Aedes aegypti. Insect Physiol. 32, 867-877. 
Share, M. R. and Roe, R. M. (1988) A partition assay for the simultaneous determination of insect juvenile hormone esterase and epoxide hydrolase activity. Anal. Biochem. 169, 81-88.

Share, M. R., Venkatesh, K., Jesudason, P. and Roe, R. M. (1988) Juvenile hormone metabolism during embryogenesis in the tobacco hornworm, Manduca sexta (L.). Arch. Insect Biochem. Physiol. 8, 173-186.

Shemshedini, L., Lanoue, M. and Wilson, T. G. (1990) Evidence for a juvenile hormone receptor involved in protein synthesis in Drosophila melanogaster. J. Biol. Chem. 265, 1913-1918.

Shemshedini, L. and Wilson, T. G. (1988) A high affinity, high molecular weight juvenile hormone binding protein in the hemolymph of Drosophila melanogaster. Insect Biochem. 18, 681-689.

Shemshedini, L. and Wilson, T. G. (1990) Resistance to juvenile hormone and an insect growth regulator in Drosophila is associated with an altered cytosolic juvenile hormone-binding protein. Proc. Natl. Acad. Sci. USA 87, 2072-2076.

Slade, M., Hetnarski, H. K. and Wilkinson, C. F. (1976) Epoxide hydrase activity and its relationship to development in the southern armyworm, Prodenia eridania. J. Insect Physiol. 22, 619-622.

Slade, M. and Zibitt, C. H. (1972) Metabolism of cecropia juvenile hormone in insects and in mammals. In Juvenile Hormones Chemistry and Action (Edited by Menn, J. J. and Boroza, M.), pp. 155-176. Academic Press, New York.

Sliter, T. J., Sedlak, B. J., Baker, F. C. and Schooley, D.A. (1987) Juvenile hormone in Drosophila melanogaster identification and titre determination during development. Insect Biochem. 17, 161-165.

Small, D. H. (1990) Non-cholinergic actions of acetylcholinesterases: proteases regulating cell growth and development? Trends Biochem. Sci. 15, 213-216.

Sokal, R. R. and Rohlf, F. J. (1969) Biometry, the principles and practice of statistics in biological research. pp. 386-387, W. H. Freeman, San Francisco.

Sparagana, S. P., Bhaskaran, G. and Barrera, P. (1985) Juvenile hormone acid methyltransferase activity in imaginal discs of Manduca sexta prepupae. Arch. Insect Biochem. Physiol. 2, 191-202.

Sparks, T. C., Allen, L. G., Schneider, F. and Granger, N. A. (1989) Juvenile hormone esterase activity from Manduca sexta corpora allata in vitro. Arch. Insect Biochem. Physiol. 11, 93-108.

Sparks, T. C. and Hammock, B. D. (1980) Comparative inhibition of the juvenile hormone esterases from Trichoplusia ni, Tenebrio molitor, and Musca domestica. Pestic. Biochem. Physiol. 14, 290-302.

Srivastava, U. S. and Gilbert, L. I. (1968) Juvenile hormone: effects in a higher dipteran. Science 161, 61-62.

Srivastava, U. S. and Gilbert, L. I. (1969) The influence of juvenile hormone on the metamorphosis of Sarcophaga bullata. J. Insect Physiol. 15, 177-189. 
Stein, S. P., Tepper, C. S., Able, N. D. and Richmond, R. C. (1984) Studies of esterase 6 in Drosophila melanogaster-XVI. Insect Biochem. 14, 527-532.

Strambi, C., Tirard, A., Renucci, M., Faure, P., Charpin, P., Augier, R. and Strambi, A. (1992) Juvenile hormone modulation of polyamine matabolism in different tissues of Acheta domesticus. In Insect Juvenile Hormone Research (Edited by Mauchamp, B., Couillaud, F. and Baehr, J. C.), pp. 193-206. Institut National de la Recherche Agronomique, Paris.

Strambi, C., Tirard, A., Renucci, M., Faure, P., Charpin, P., Augier, R. and Strambi, A. (1993) Ecdysone deprivation affects polyamine metabolism in the house cricket Acheta domesticus. J. Insect Biochem. Molec. Biol. 23, 165-170.

Strand, M. R., Goodman, W. G. and Baehrecke, E. H. (1991) The juvenile hormone titer of Trichoplusia $n i$ and its potential role in embryogenesis of the polyembryonic wasp Copidosoma floridanum. Insect Biochem. 21, 205-214.

Stryer, L. (1981) Biochemistry. p. 115. W.H. Freeman, San Francisco.

Szolajska, E. (1991) Hydrolytic degradation of juvenile hormones in haemolymph and corpora allata of Galleria mellonella (L). Acta Biochimica Polonica 36, 321-333.

Tanaka, S., Ming, T. C., Denlinger, D. L. and Abdel-Aal, Y. A. I. (1989) Developmental landmarks and the activity of juvenile hormone and juvenile hormone esterase during the last stadium and pupa of Lymantria dispar. J. Insect Physiol. 35, $897-$ 905.

Tata, J. R. (1993) Gene expresion during metamorphosis: an ideal model for post embryonic development. BioEssays 15, 239-248.

Tobe, S. S., Ruegg, R. P., Stay, B. A., Baker, F. C., Miller, C. A. and Schooley, D. A. (1985) Juvenile hormone titre and regulation in the cockroach Diploptera punctata. Experientia 41, 1028-1034.

Tobe, S. S. and Stay, B. (1985) Structure and regulation of the corpus allatum. Adv. Insect Physiol. 18, 305-432.

Trowell, S. C. (1992) High affinity juvenile hormone carrier proteins in the haemolymph of insects. Comp. Biochem. Physiol. 103B, 795-807.

Valaitis, A. P. (1991) Characterisation of the hemolymph juvenile hormone esterase from Lymantria dispar. Insect Biochem. 21, 583-595.

Valaitis, A. P. (1992) Use of concanavalin-A in the purification of juvenile hormone esterase from the hemolymph and the fat body of Lymantria dispar. Insect Biochem. Molec. Biol. 22, 639.

Venkatesh, K., Abdel-Aal, Y. A. I., Armstrong, F. B. and Roe, R. M. (1990) Characterisation of affinity purified juvenile hormone esterase from the plasma of the tobacco hornworm, Manduca sexta. J. Biol. Chem. 265, 21727-21732.

Venkatesh, K., Crawford, C. L. and Roe, R. M. (1988) Characterisation and the developmental role of plasma juvenile hormone esterase in the adult cabbage looper, Trichoplusia ni. Insect Biochem. 18, 53-61. 
Vernick, K. D., Collins, F. H., Seeley, D. C., Gwadz, R. W. and Miller, L. H. (1988) The genetics and expression of an esterase locus in Anopheles gambiae. Biochem. Genet. 26, 367-379.

Ward, V. K., Bonning, B. C., Huang, T., Shiotsuki, T., Griffeth, V. N. and Hammock, B. D. (1992) Analysis of the catalytic mechanism of juvenile hormone esterase by sitedirected mutagenesis. Int. J. Biochem. 24, 1933-1941.

Watson, R. D., Agui, N., Haire, M. E. and Bollenbacher, W. E. (1987) Juvenile hormone coordinates the regulation of the hemolymph ecdysteroid titer during pupal commitment in Manduca sexta. Insect Biochem. 17, 955-959.

Weirich, G. and Wren, J. (1976) Juvenile-hormone esterase in insect development: A comparative study. Physiol. Zool. 49, 341-350.

White, M. M., Mane, S. D. and Richmond, R. C. (1988) Studies of esterase 6 in Drosophila melanogaster. XVIII. biochemical differences between the slow and fast allozymes. Mol. Biol. Evol. 5, 41-62.

Whitmore, C. F., Whitmore, E. and Gilbert, L. I. (1972) Juvenile hormone induction of esterases: a mechanism for the regulation of juvenile hormone titre. Proc. Natl. Acad. Sci. U.S.A. 69, 1592-1595.

Wigglesworth, V. B. (1954) The physiology of insect metamorphosis. Cambridge University Press, Cambridge.

Willis, J. H. (1981) Juvenile hormone: the status of "status quo". Amer. Zool. 21, 763-773.

Willis, J. H. (1990) Regulating genes for metamorphosis: concepts and results. In Molecular Insect Science (Edited by Hagedorn, H. H., Hildebrand, J. G., Kidwell, M. G. and Law, J. H.), pp.91-98 Plenum Press, New York.

Willis, J. H. and Cox, D. L. (1984) Defining the anti-metamorphic action of juvenile hormone. In Biosynthesis, Metabolism and Mode of Action of Invertebrate Hormones (Edited by Hoffman, J. and Porchet, M.), pp. 465-474. Springer-Verlag, Berlin Heidelberg.

Wilson, T. G. and Gilbert, L. I. (1978) Metabolism of juvenile hormone I in Drosophila melanogaster. Biochem. Physiol. 60A, 85-89.

Wing, K. D., Rudnicka, M., Jones, G., Jones, D. and Hammock, B. D. (1984) Juvenile hormone esterases of Lepidoptera II. Isoelectric points and binding affinities of hemolymph juvenile hormone esterase and binding protein activities. J. Comp. Physiol. B. 154, 213-223.

Wisniewski, J. R., Muszynska-Pytel, M., Grzelak, K. and Kochman, M. (1987) Biosynthesis and degradation of juvenile hormone in corpora allata and imaginal wing discs of Galleria mellonella (L.). Insect Biochem. 17, 249-254.

Wisniewski, J. R., Muszynska-Pytel, M. and Kochman, M. (1986b) Juvenile hormone degradation in brain and corpora cardiaca-corpora allata complex during the last larval instar of Galleria mellonalla (Lepidoptera, Pyralidae). Experientia 42, 167169. 
Wisniewski, J. R., Rudnicka, M. and Kochman, M. (1986a) Tissue specific juvenile hormone degradation in Galleria mellonella. Insect Biochem. 16, 843-849.

Wozniak, M. and Jones, D. (1987) Immunochemical characterisation of juvenile hormone esterase from different species of Lepidoptera. Biochem. Biophys. Res. Comm. 144, 1281-1286.

Wozniak, M. and Jones, G. (1990) Glycosylation and isoform variation of juvenile hormone esterase in the fat body and hemolymph during metamorphosis of Trichoplusia ni (Lepidoptera). Molec. Cell. Endocrinol. 70, 255-262.

Wozniak, M., Jones, G., Hiremath, S. and Jones, D. (1987) Biochemical and immunological properties of different forms of juvenile hormone esterase from Trichoplusia ni (Hübner). Biochim. Biophys. Acta 926, 26-39.

Wright, C. I., Geula, C. and Mesulam, M. (1993) Protease inhibitors and indoleamines selectively inhibit cholinesterases in the histopathologic structures of Alzheimer disease. Proc. Natl. Acad. Sci. USA 90, 683-686.

Wyatt, G. R., Cook, K. E., Firko, H. and Dhadialla, T. S. (1987) Juvenile hormone action on locust fat body. Insect Biochem. 17, 1071-1073.

Yamomoto, K., Chadarevian, A. and Pellegrini, M. (1988) Juvenile hormone action mediated in male accessory glands of Drosophila by calcium and kinase C. Science 239, 916-919.

Yu, S. J. and Terriere, L. C. (1978a) Metabolism of juvenile hormone I by microsomal oxidase, esterase, and epoxide hydrase of Musca domestica and Sarcophaga bullata. Pestic. Biochem. Physiol. 9, 237-246.

Yu, S. J. and Terriere, L. C. (1978b) Juvenile hormone epoxide hydrase in house flies, flesh flies and blow flies. Insect Biochem. 8, 349-352.

Yuhas, D. A., Roe, R. M., Sparks, T. C. and Hammond, A. M. (1983) Purification and kinetics of juvenile hormone esterase from the cabbage looper, Trichoplusia ni (Hübner). Insect Biochem. 13, 129-136.

Zdárek, J. and Denlinger, D. L. (1987) Pupal ecdysis in flies: the role of ecdysteroids in its regulation. J. Insect Physiol. 33, 123-128.

Zera, A. J., Gu, X. and Zeisset, M. (1992) Characterisation of juvenile hormone esterase from genetically-determined wing morphs of the cricket, Gryllus rubens. Insect Biochem. Molec. Biol. 22, 829-839.

Zera, A. J. and Holtmeier, C. L. (1992) In vivo and in vitro degradation of juvenile hormone-III in presumptive long-winged and short-winged Gryllus rubens. J. Insect Physiol. 38, 61-74.

Zimowska, G., Rembold, H. and Bayer, G. (1989) Juvenile hormone identification, titer, and degradation during the last larval stadium of Spodoptera littoralis. Arch. Insect Biochem. Physiol. 12, 1-14. 


\section{Appendix}

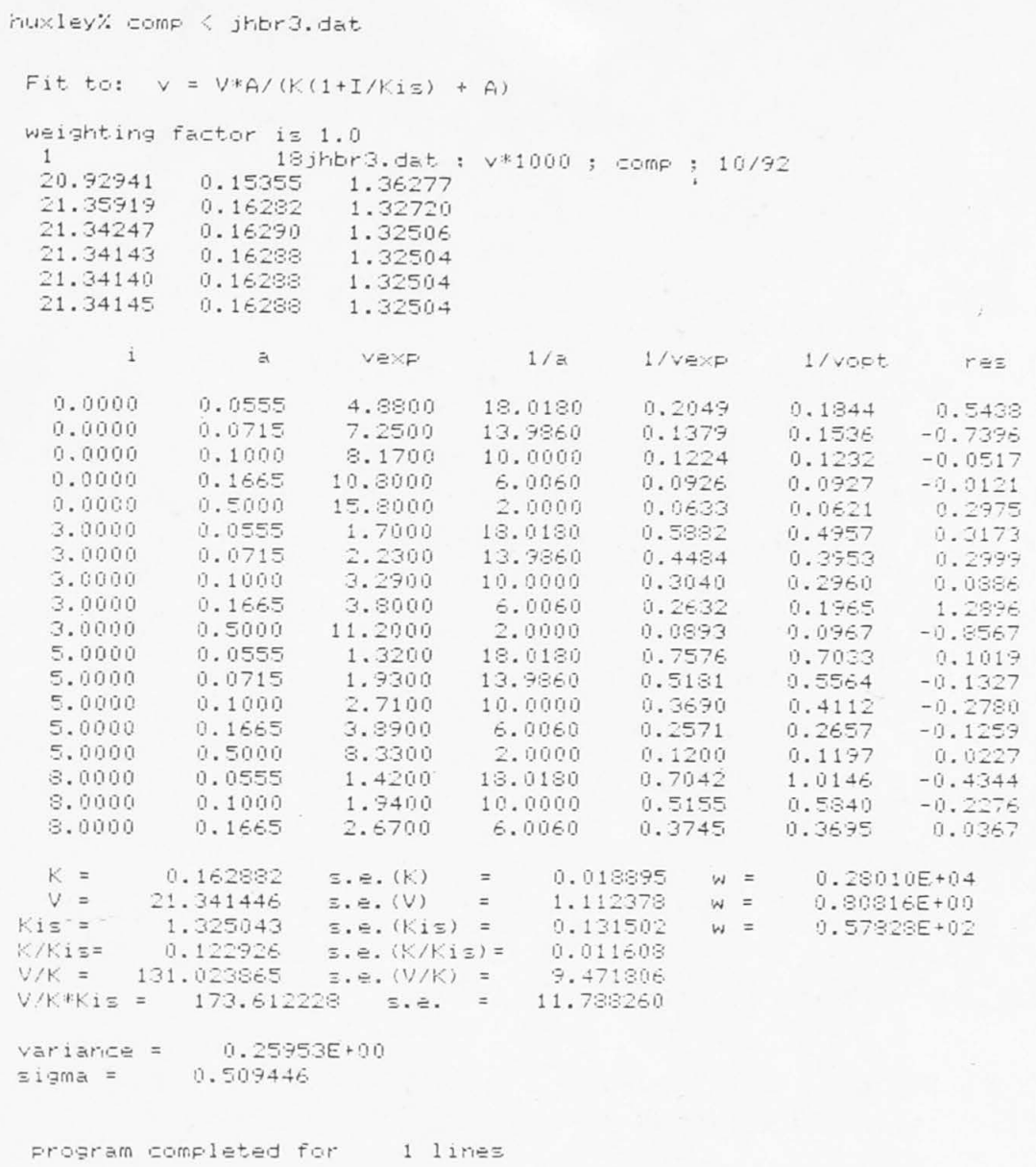

Veeglited mean $K_{15}=1.20 \pm 0.09$, $2 \mathrm{~m}$.

Shown above is an example of the computer analysis of the data shown in figure 3.8. The absolute values of the reaction rates are different because the data shown in figure 3.8 has been corrected for loss of enzyme activity during storage (Section 3.2.4.1). 


\section{Batch 7}

Number -386

RECORDS \# - b18568373

TITLE - Characterisation of juvenile hormone esterase in Drosophila melanogaster / Peter Malcolm

Campbell

AUTHOR - Campbell, Peter Malcolm

PUB - 1993

CALL \# - QL537.D76C36 1993

BARC - +1926149

VOL -

NO. OF PAGES - 130

RESTRICTION - Open

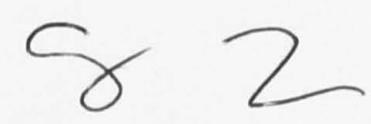

